\title{
Evaluation of cholesterol-lowering and antioxidant properties of sugar cane policosanols in hamsters and humans
}

\author{
Amira N. Kassis \\ School of Dietetics and Human Nutrition \\ McGill University, Montreal
}

April 2008

A thesis submitted to McGill University in partial fulfillment of the requirements of the degree of Doctorate of Philosophy

(C) Amira N. Kassis 2007 


\section{Abstract}

Atherosclerosis prevention is now a major focus of the scientific community and the pharmaceutical industry. Sugar cane policosanols (SCP) have gained increasing popularity over the last decade as a result of numerous studies conducted in Cuba considering SCP as the natural alternative to statin drugs. However, independent research on policosanols was not able to replicate cholesterol reductions reported by Cuban laboratories. No independent study to date has examined the cholesterol-lowering effect and antioxidant capacity of original SCP in humans. In addition, since independent research was criticized because of the use of alternative policosanol formulations, the source and composition of policosanol mixtures are now at the core of the policosanol controversy.

The aim of the present thesis project was first to compare the composition and cholesterol-lowering effects of different SCP preparations in hamsters, and secondly to test the cholesterol-lowering efficacy, mechanism of action and antioxidant capacity of the original Cuban SCP in hypercholesterolemic humans.

Study 1: Forty-eight male hamsters were randomly assigned to 4 groups for a period of 4 weeks (i) non-cholesterol control, (ii) cholesterol control, (iii) original SCP and (iv) alternative SCP. Hamsters were sacrificed and blood was collected at the end of the feeding period for lipid measurements.

Study 2: Twenty-one hypercholesterolemic volunteers consumed $10 \mathrm{mg} /$ day of SCP or a placebo for a period of 28 days in a crossover trial. Plasma lipid levels and LDL oxidation were measured at the start and end of supplementation phases. Cholesterol absorption and synthesis were assessed using single isotope single tracer technique and deuterium incorporation respectively.

There was no significant difference in cholesterol-lowering efficacy between hamsters in the original and alternative SCP groups relative to control. Similarly, in hypercholesterolemic humans, original SCP supplementation did not significantly improve lipid parameters and no change in cholesterol absorption or synthesis was observed relative to control. In vivo assessment of LDL oxidation showed no significant changes in oxidized LDL concentration relative to baseline and control. 
This thesis disagrees with previous Cuban data on the cardio-protective role of SCP in animals and humans and supports independent studies showing no cholesterol-lowering effect and no antioxidant capacity. 


\section{Résumé}

L'athérosclérose est actuellement la cible principale de la communauté scientifique ainsi que les companies pharmaceutiques. Les policosanols issus de la cane à sucre (SCP) ont gagné une importance majeure suite à une série d'études effectuées à Cuba et montrant que ces substances représentaient l'alternative naturelle aux statines. Cependant, des laboratoires indépendants ont récemment refuté les conclusions des chercheurs Cubains, n'ayant pas observé d'amélioration du profile lipidique suite à une supplémentation de SCP. Aucune étude indépendante n'a entrepris de mesurer les effets hypolipémiants et antioxidants des SCP dans une population hypercholestérolémique. De plus, les chercheurs indépendants ayant été critiques pour leur utilisation de préparations alternatives, la source et la composition des formulations de policosanols est actuellement au sein de la controverse sur les effets de ces statines naturelles.

Les objectifs du projet de thèse présent était premièrement de comparer la composition de différentes sortes de policosanols ainsi que leurs effets réducteurs sur le niveau du cholestérol sanguins chez les hamsters, et deuxièmement d'évaluer la preparation de SCP originale en terme de réduction de cholestérol, méchanisme d'action, et pouvoir antioxidant, et cela chez des individus hypercholestérolémiques.

Etude 1: Quarante-huit hamsters ont été randomisés pour recevoir 4 traitements differents (i) contrôle sans cholestérol (ii) contrôle avec cholestérol (iii) SCP originaux et (iv) SCP alternatifs. Les hamsters ont été sacrifiés et leur plasma a été utilisé pour la determination du profile lipidique.

Etude 2: Vingt et un volontaires hypercholestérolémiques ont consommé $10 \mathrm{mg}$ de SCP/ jour ou un placebo pour une période de 28 jours, dans le cadre d'une étude croisée. Le bilan lipidique ainsi que l'oxidation des lipoprotéines à basse densité (LDL) ont été mesurés au début et à la fin des phases de supplementation. La technique de simple isotope stable et d'incorporation de deutérium ont été utilisés afin de déterminer l'absorption intestinale et la synthèse du cholestérol respectivement.

Aucune différence significative n'a été observée en terme de réduction du cholesterol chez les hamsters recevant les SCP originaux et alternatifs relativement au contrôle. De même, chez les sujets hypercholestérolémiques, la supplementation de SCP 
n'a pas causé d'amélioration du profile lipidique et aucun changement n'a été noté en terme d'absorption et synthèse du cholestérol, relativement au placebo. L'analyse in vivo de l'oxidation des LDL a démontré que la concentration d'LDL oxidés dans le plasma des sujets était similaire suite à la supplémentation de SCP relativement au niveau de base et au contrôle.

Le projet de thèse present est en désaccord avec les résultats issus des recherches précédentes effectuées par les groupes de recherche Cubains sur les effets cardioprotecteurs des SCP chez les animaux et les humains. Au contraire, nos resultats soutiennent les conclusions déduites par des chercheurs indépendants n'ayant pas observé d'effets sur le niveau de cholestérol sanguin ou le stress oxidatif. 


\section{Acknowledgments}

I would like to thank the people who, throughout my time at McGill, contributed towards making my $\mathrm{PhD}$ work a wonderful experience. First and foremost, my supervisor Dr. Peter Jones who has provided guidance, demonstrated patience and support throughout, but mostly for giving me great opportunities in the field of nutrition research. I am thankful to Catherine Vanstone for teaching me most of what I know about clinical trials during my Masters, to Sylvia Santosa and Stephanie Jew for being great friends and for their support and help during stressful times. I am also grateful to the friends I had the chance to make at McGill, who made this experience so much more enjoyable, most importantly Suhad Abumweis for her work and life advice, Scott Harding for his help, Lindiwe Sibeko and Patrick Owen for creating a great supporting environment for thesis writing in our $\mathrm{PhD}$ office and beyond. Special thanks to Christopher Marinangeli for making the hamster trial so much easier to handle and for being there for me all along in every way possible. Last but not least, I would like to thank my parents, Nemr and Valerie Kassis who stuck by me and by every decision I made during my years at McGill and who supported me unconditionally, regardless of how long and far I had to be away from them. 


\section{Contribution of authors}

Manuscript 1: Lack of effect of sugar cane policosanols on plasma cholesterol in golden Syrian hamsters

Amira N. Kassis, Christopher P.F. Marinangeli, Deepak Jain, Naoyuki Ebine, Peter J.H. Jones

Amira Kassis, the candidate, was involved in developing the study design, running the animal trial, collecting and analyzing data. Amira also wrote and submitted Manuscript 1 for publication.

Christopher Marinangeli, a fellow graduate student in Dr. Peter Jones' lab, was involved in running the hamster trial and collecting data. Christopher provided useful feedback on Manuscript 1.

Dr. Deepak Jain, a graduate student in Dr. Peter Jones' lab participated in the design of the hamster study and data collection during the trial. He also provided feedback on Manuscript 1.

Dr. Naoyuki Ebine, a post-doctoral fellow in Dr. Peter Jones' lab, was involved in the design of the hamster trial, participated in the collection of data and contributed his input to Manuscript 1.

Dr. Peter Jones, the candidate supervisor, designed the study protocol of the hamster trial and provided guidance and support during data collection and manuscript writing. 
Manuscript 2: Comparison of composition and absorption of sugar cane policosanols Christopher P.F. Marinangeli, Amira N. Kassis, Deepak Jain, Naoyuki Ebine, Stephen C. Cunnane, Peter J.H. Jones

Christopher Marinangeli was involved in developing the study design, running the animal trial as well as collecting and analyzing data. Christopher also wrote and submitted Manuscript 2 for publication.

Amira Kassis was involved in running the hamster trial and collecting data. Amira provided useful feedback on Manuscript 2.

Dr. Deepak Jain participated in the design of the hamster study and data collection during the trial. He also provided feedback on Manuscript 2.

Dr. Naoyuki Ebine was involved in the design of the hamster trial, participated in the collection of data and contributed his input to Manuscript 2.

Dr. Stephen Cunnane, Professor of Medicine and Physiology at the University of Sherbrooke, provided laboratory space and equipment for sample analysis as well as guidance in methodology. Dr. Cunnane contributed useful input to Manuscript 2.

Dr. Peter Jones designed the study protocol of the hamster trial and provided guidance and support during data collection and manuscript writing. 
Manuscript 3: Lack of cholesterol-lowering efficacy of Cuban sugar cane policosanols in hypercholesterolemic persons

Amira N. Kassis and Peter J.H. Jones

Amira Kassis was involved in developing the study design of the human trial. Amira ran the study and laboratory experiments, collected and analyzed data then wrote and submitted Manuscript 3 for publication.

Dr. Peter Jones designed the study protocol of the human trial and provided guidance and support during data collection and manuscript writing.

Manuscript 4: Changes in cholesterol synthesis and absorption following sugar cane policosanol supplementation in hypercholesterolemic individuals

Amira N. Kassis and Peter J.H Jones

Amira Kassis was involved in developing the study design of the human trial. Amira ran the study and laboratory experiments, collected and analyzed data and wrote Manuscript 4.

Dr. Peter Jones designed the study protocol of the human trial and provided guidance and support during data collection and manuscript writing.

Manuscript 5: Lack of effect of Cuban sugar cane policosanols on LDL oxidation in hypercholesterolemic individuals Amira N. Kassis, Stan Kubow and Peter J.H. Jones

Amira Kassis was involved in developing the study design of the human trial. Amira ran the study and laboratory experiments, collected and analyzed data and wrote Manuscript 5 . 
Dr. Stan Kubow, Associate Professor in the School of Dietetics and Human Nutrition at McGill University, provided guidance in the choice of method for oxidative stress measurement. Dr. Kubow contributed usefull input to Manuscript 5.

Dr. Peter Jones designed the study protocol of the human trial and provided guidance and support during data collection and manuscript writing.

Editorial: Policosanols lose their luster

Peter J.H. Jones, Amira N. Kassis and Christopher P.F. Marinangeli

Peter Jones wrote the manuscript.

Amira Kassis contributed to the writing and editing of the manuscript.

Christopher Marinangeli contributed to the writing and editing of the manuscript. 


\section{Table of contents}

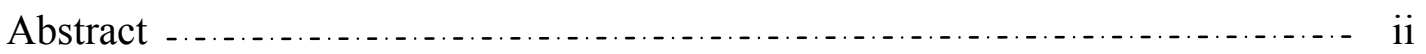

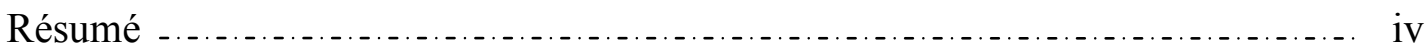

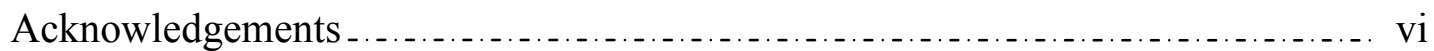

Contributions of authors $\ldots \ldots \ldots \ldots \ldots \ldots \ldots \ldots$

List of abbreviations $\ldots \ldots \ldots \ldots \ldots \ldots \ldots$

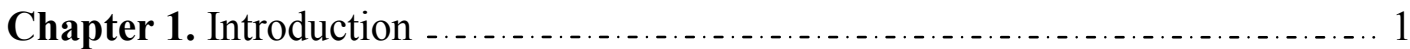

1.1. Background …......................................... 1

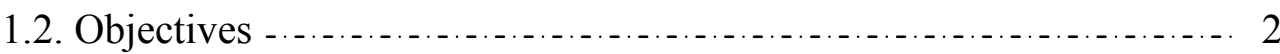

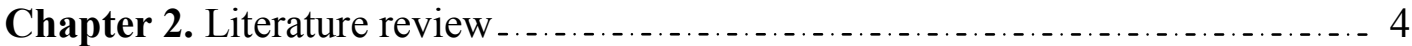

2.1. Policosanols: Structure, sources and properties _................... 4

2.2. Lipid lowering effect of policosanols _............................ 4

2.2.1. Animal studies investigating policosanol efficacy ............ 4

2.2.2. Human studies investigating policosanol efficacy _.......... 6

2.3. Effect of policosanols on cholesterol metabolism_................... 9

2.4. Effect of sugar cane policosanols on lipid peroxidation .............. 12

2.5. Composition and metabolism of policosanol mixtures _............... 14

2.6. Safety and tolerability of sugar cane policosanols _...................15

2.7. Summary and conclusions....................................... 16

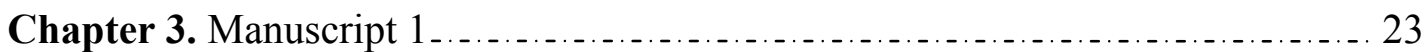

Lack of effect of sugar cane policosanols on plasma cholesterol in golden Syrian hamsters

Manuscript outline

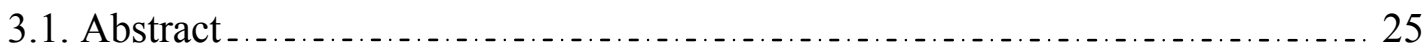

3.2. Introduction

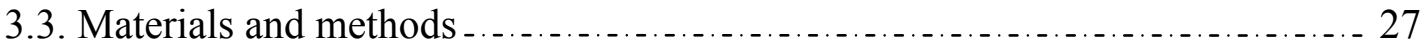

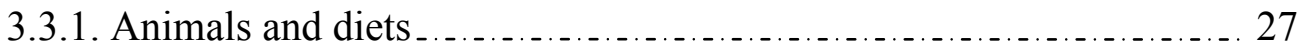

3.3.2. Protocol

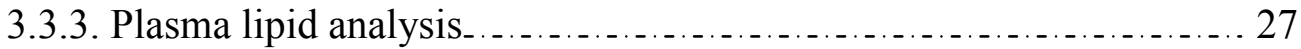

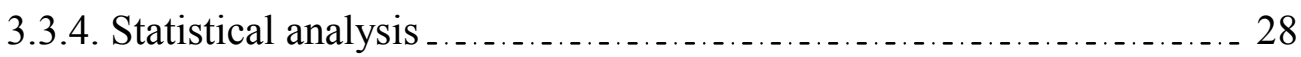


3.4. Results

3.4.1. Growth of hamsters across the study period _.................... 28

3.4.2. Food intake of hamsters throughout the feeding period .............. 28

3.4.3. Growth and intake of hamsters versus plasma lipids _............. 29

3.4.4. Lipid values as a result of policosanol feeding _.................. 29

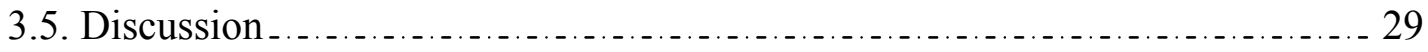

3.6. Conclusion

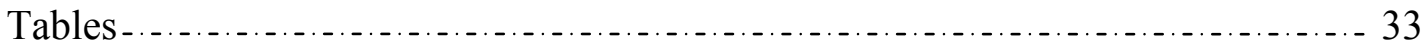

Figures

Bridge 1 1

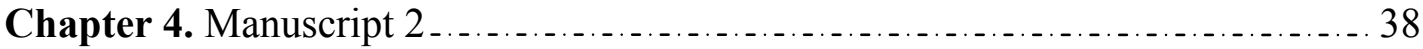

Comparison of composition and absorption of sugar cane policosanols

Manuscript outline 39

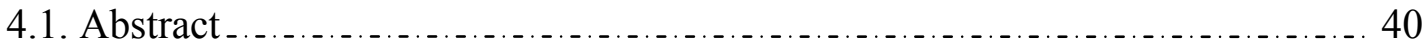

4.2. Introduction

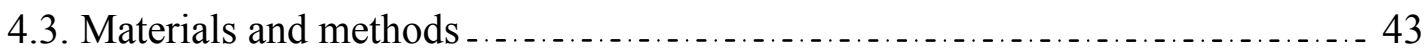

4.3.1. Compositional analysis _...................................... 43

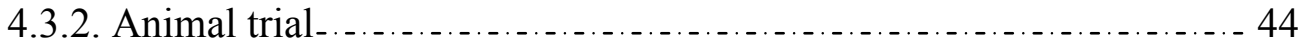

4.3.2.1. Animals and diets_....................................... 44

4.3.2.2. Tissue and plasma analysis _............................. 45

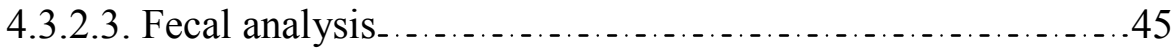

4.3.2.4. Gas chromatography analysis_............................. 45

4.3.3. Statistical analysis _............ 46

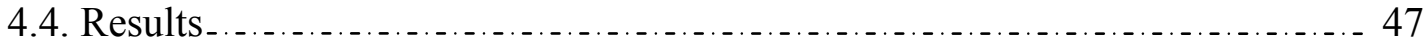

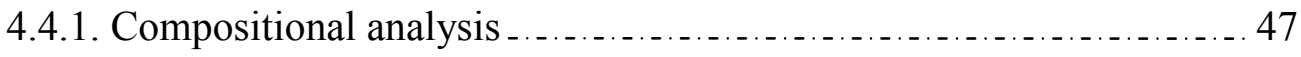

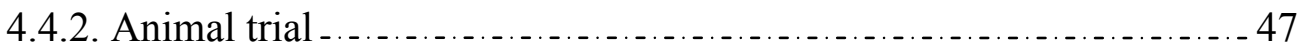

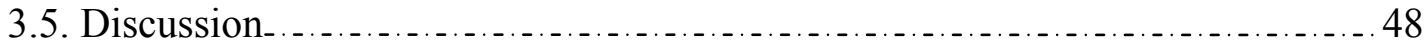

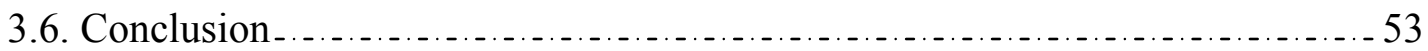

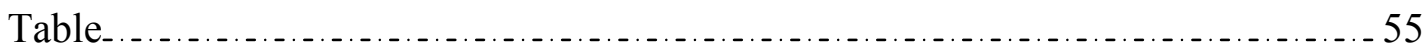

Figure

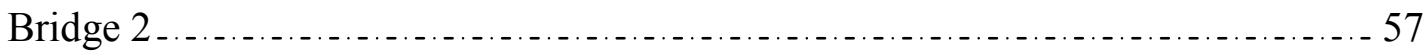




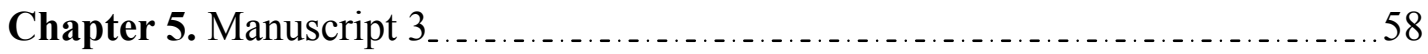

Lack of cholesterol-lowering efficacy of Cuban sugar cane policosanols in hypercholesterolemic persons

Manuscript outline

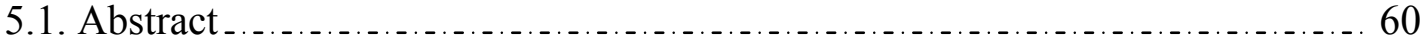

5.2. Introduction

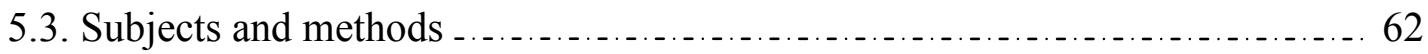

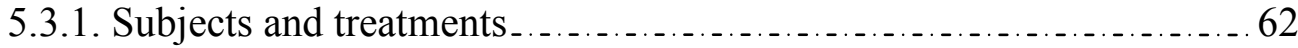

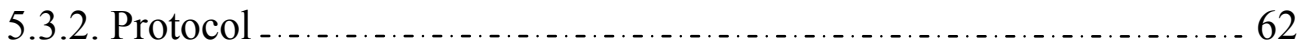

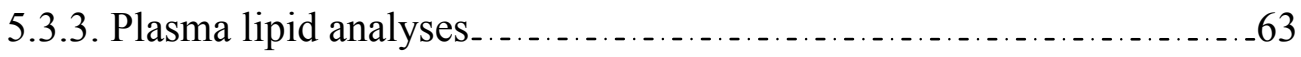

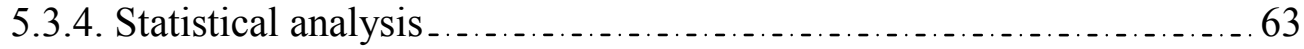

5.4. Results

5.4.1. Subjects and drop-out rate 64

5.4.2. Blood biochemistry and weight changes across the study period _.... 64

5.4.3. Plasma lipid levels across the study period_.................................

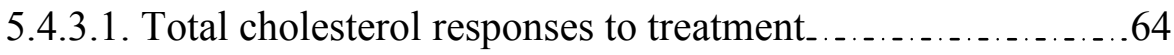

5.4.3.2. LDL-cholesterol responses to treatment ..................64

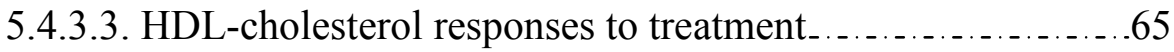

5.4.3.4. Triacylglycerol responses to treatment _................... 65

5.4.3.5. Effect of sequence, period and body weight _............. 65 change on lipid parameters

5.5. Discussion

5.6. Conclusion _... 69

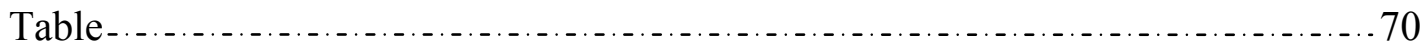

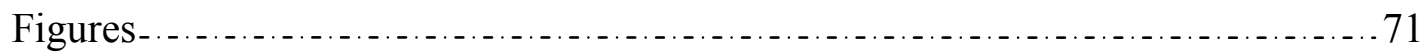

Bridge 3 _... 76

Chapter 6. Manuscript $4 \ldots \ldots \ldots$

Changes in cholesterol absorption and synthesis following sugar cane policosanol supplementation

Manuscript outline 


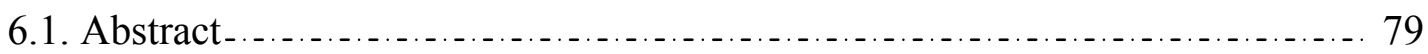

6.2. Introduction $\ldots$

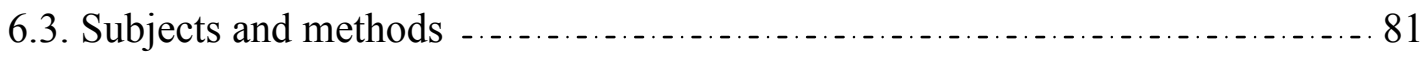

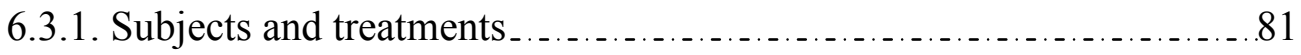

6.3.2. Protocol

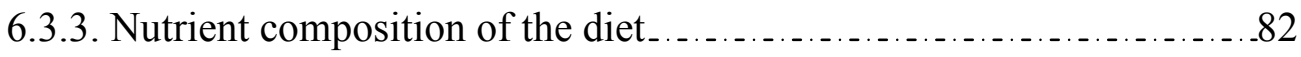

6.3.4. Determination of cholesterol absorption _............................ 83

6.3.5. Determination of cholesterol biosynthesis _.......................... 83

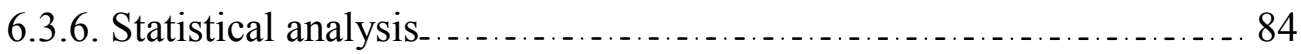

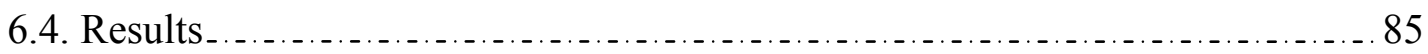

6.4.1. Compliance and drop-out rate_................................... 85

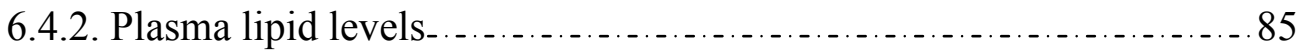

6.4.3. Dietary intake throughout the study period _......................... 85

6.4.4. Cholesterol absorption as a result of SCP supplementation ......... 86

6.4.5. Cholesterol synthesis as a result of SCP supplementation ........... 86

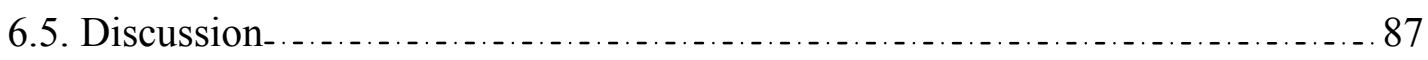

6.6. Conclusion

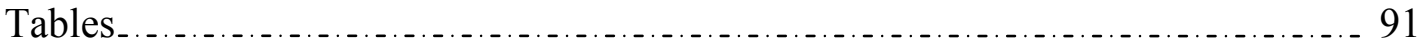

Figures_...

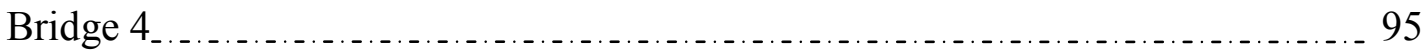

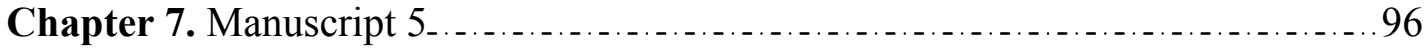

Lack of effect of Cuban sugar cane policosanols on LDL oxidation in hypercholesterolemic individuals

Manuscript outline

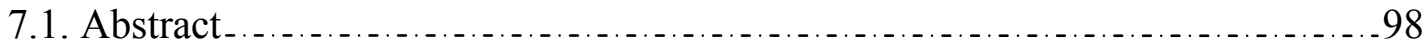

7.2. Introduction $\ldots \ldots \ldots \ldots$

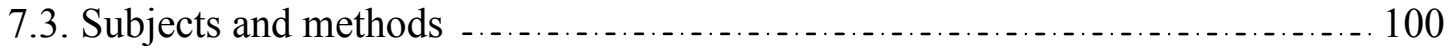

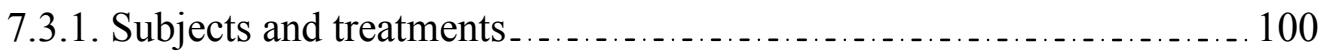

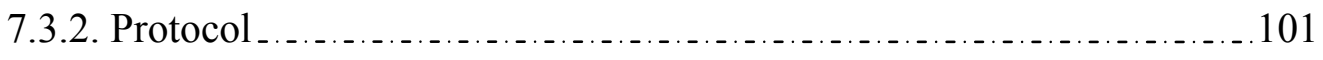

7.3.3. LDL oxidation measurements _................................. 101

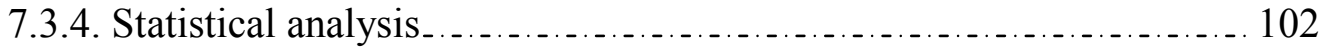




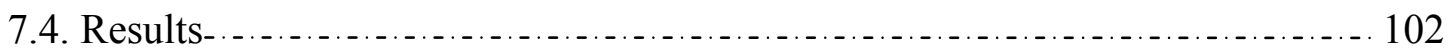

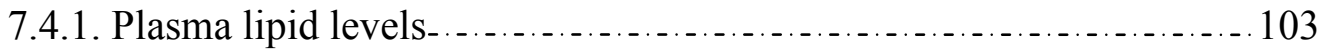

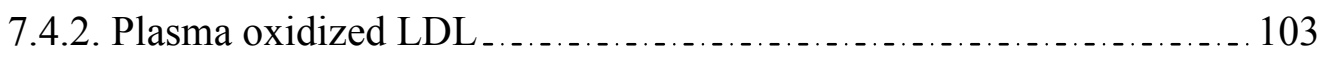

7.5. Discussion

7.6. Conclusion

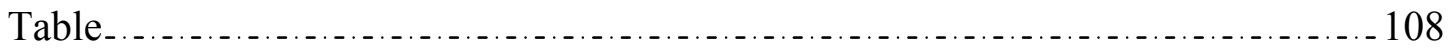

Figures_... 109

Bridge 5

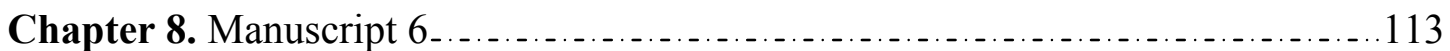

Editorial- Policosanols lose their lustre

Chapter 9. Discussion and conclusions.

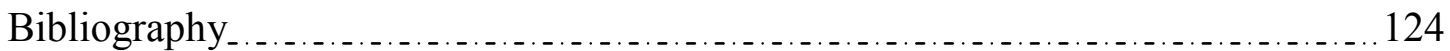

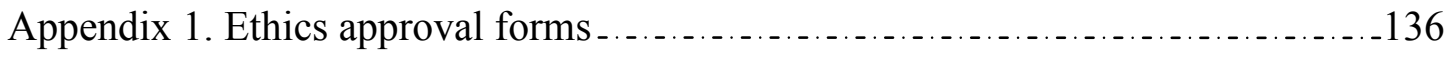

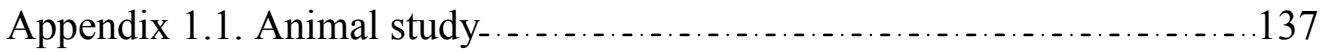

Appendix 1.2. Human study_.......................................... 146

Appendix 2. Subject consent form $\ldots \ldots \ldots \ldots \ldots \ldots$

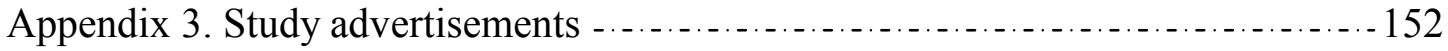

Appendix 3.1. English copy _............................... 152

Appendix 3.2. French copy

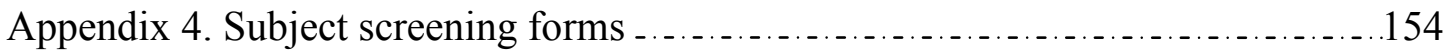

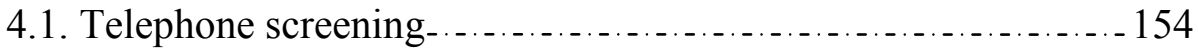

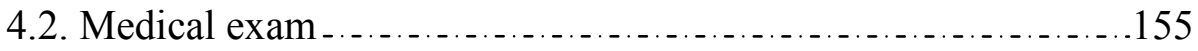

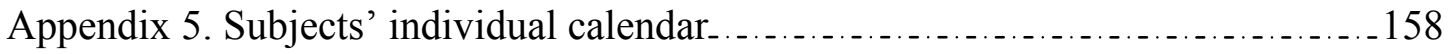

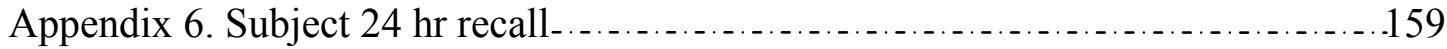

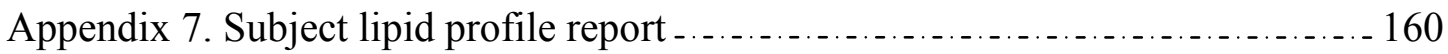

Appendix 8. Manuscripts included in thesis _.............................. 162

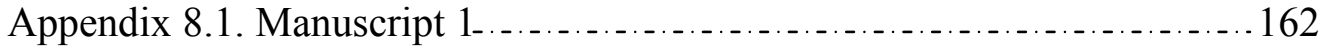

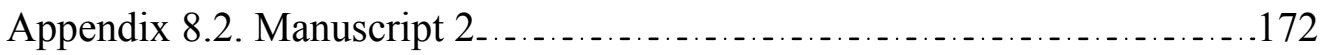

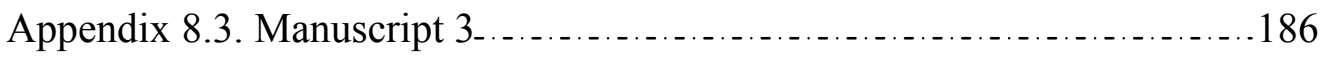

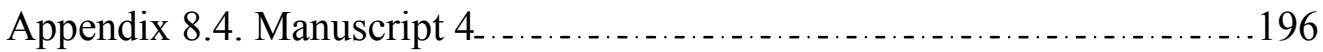

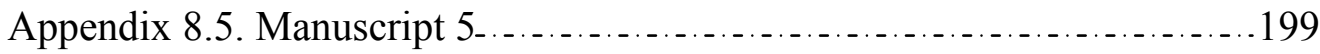




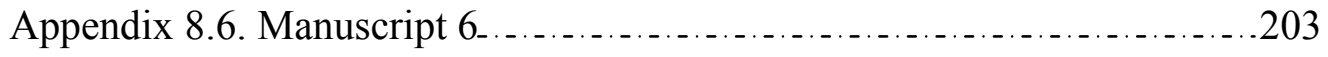




\section{List of tables}

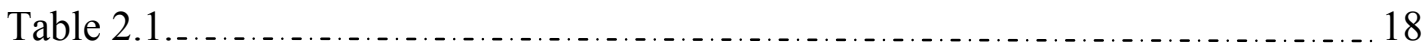

Composition of original sugar cane policosanol

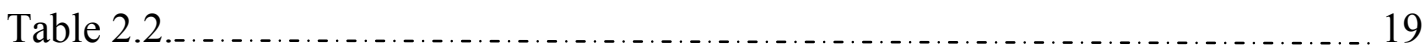

Recent policosanol supplementation studies conducted by original (1) and independent (2) research groups.

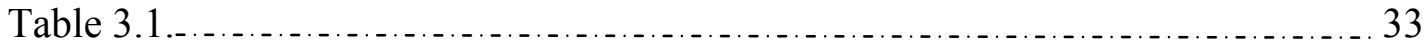

Composition of the hamster diet

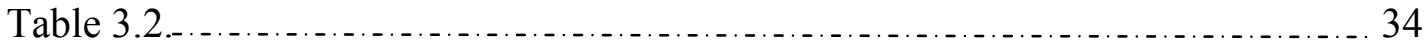

Plasma lipids in response to policosanol diets as compared to controls

Table 4.1._. 55

Comparison of the published composition of the OPC supplement to both OPC

supplements sold in difference countries under various brand names and various APC supplements

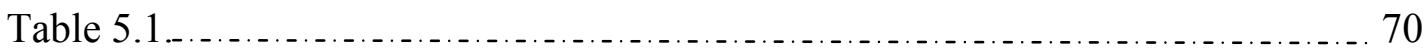

Baseline characteristics of subjects

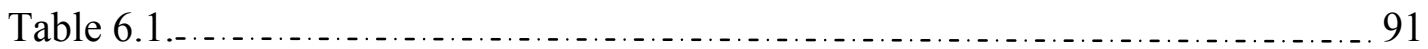

Baseline characteristics of subjects

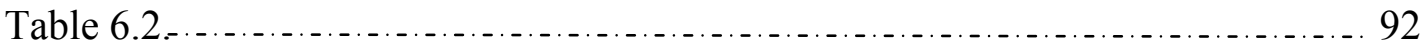

Diet composition of subjects throughout study period

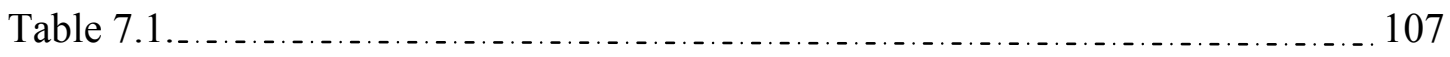

Baseline characteristics of subjects 


\section{List of figures}

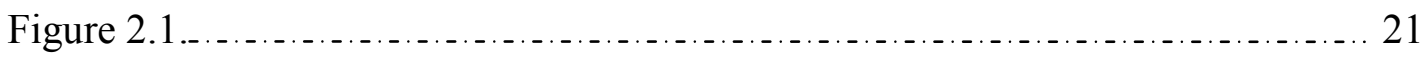

Effect of sugar cane policosanols (SCP) on hepatic cholesterol biosynthesis

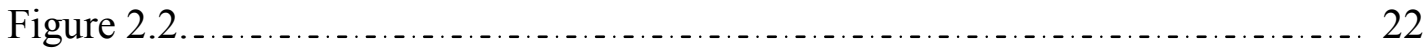

Reduction of LDL-cholesterol oxidation as a result of SCP supplementation

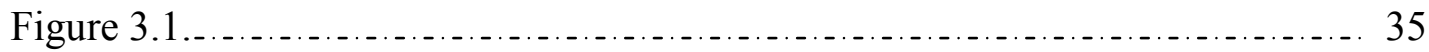

Body weights across the feeding period

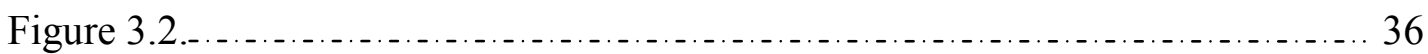

Average intake of hamsters in different diet groups throughout the study period

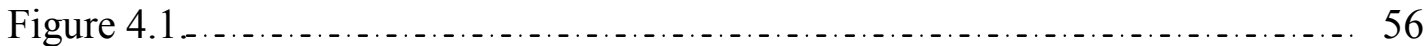

Comparison of the ratio of the average 3-day fecal excretion of octacosanol to octacosanol consumption.

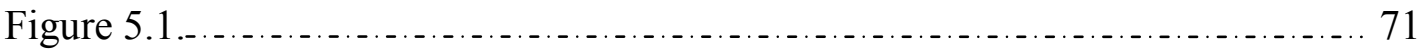

Average initial and final body weights across treatment phases

Figure 5.2.1. 72

Subjects' plasma lipid endpoints in response to SCP treatment and control

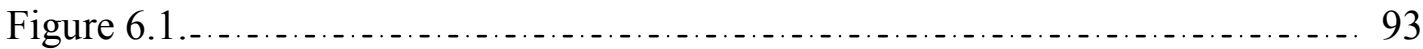

${ }^{13} \mathrm{C}$ enrichment of RBCs after isotope administration in SCP and control groups

Figure 6.2.

Difference in ${ }^{13} \mathrm{C}$ enrichment between time points and hour 0 as a result of isotope administration in SCP and control groups

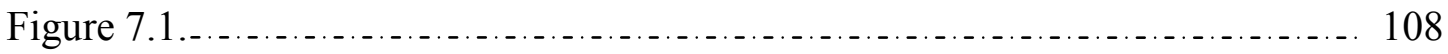

Changes in oxidized LDL across treatment periods

Figure 7.2.1. 109

Regression of Oxidized LDL concentration and LDL cholesterol levels in plasma

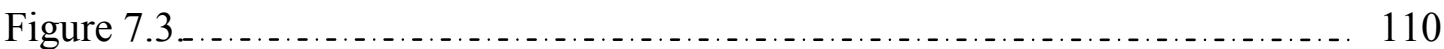

Regression of change in oxidized LDL on change in LDL levels in plasma 


\section{Abbreviations}

AIN: American institute of nutrition

ALT: alanine aminotransferase

AMP: adenosine monophosphate

ANOVA: analysis of variance

ANCOVA: analysis of covariance

APC: alternative policosanols

ARF: atherosclerotic risk factor

AST: aspartate aminostransferase

AUC: area under the curve

BMI: body mass index

C28: 28 carbons

CC: cholesterol control

CPK: creatinine phosphokinase

CSCP: Cuban sugarcane policosanols

CVD: cardiovascular disease

DM: type 2 diabetes

EDTA: ethylenediamine tetraacetic acid

ELISA: enzyme-linked immunosorbent assay

FSR: fractional rate of synthesis

GC: gas chromatography

MS: mass spectrometry

HC: type II Hypercholesterolemic

HDL: high-density lipoprotein

HL: hyperlipidemia

HMG-CoA: 3-hydroxy-3-methylglutaryl- coenzyme A

IRMS: isotope ratio mass spectrometry

LDL: low-density lipoprotein

MDA: malonaldehyde

$\mathrm{NC}$ : normocholesterolemic

NCC: non cholesterol control 
NCEP: National Cholesterol Education Program

OPC: original policosanols

PAD: peripheral artery disease

PC: policosanols

PMW: postmenopausal women

PW: plasma water

RBC: red blood cell

RPC: rice policosanols

SCP: sugar cane policosanol

SEM: standard error of the mean

TBARS: thiobarbituric acid reactive substances

TC: total cholesterol

TC-EA: high temperature conversion elemental analyzer

TG: triglycerides

V-BDP: vienna peedee belemnite

VLCFA: very long chain fatty acid

V-SMOW: vienna standard mean ocean water

WGP: wheat germ policosanols 


\section{Chapter 1}

\section{Introduction}

\subsection{Background}

Elevated low-density lipoprotein cholesterol (LDL-C) has been identified as a primary risk factor for cardiovascular disease (CVD), the major cause of death in western societies. In an effort to prevent high serum LDL- cholesterol (LDL-C), aggressive diet therapy and physical activity are recommended for individuals with or at high risk of CVD (Pearson et al., 2002). However, in many cases drug therapy is necessary (Hunninghake et al., 1993) although conventional lipid-lowering drugs such as 3hydroxy-3-methylglutaryl- coenzyme A (HMG-CoA)-reductase inhibitors or statins have been associated with serious side effects (Schachter, 2005). This explains the increasing wariness of the public towards aggressive drug therapy and the growing interest in emerging natural alternatives.

Policosanols are mixtures of higher aliphatic alcohols found in a variety of plant foods including whole grains, nuts and unrefined plant oils (Hargrove et al., 2004) and have been shown to improve major indicators of cardiovascular health, namely blood cholesterol and LDL oxidation status. Animal and human studies have demonstrated that policosanol administration safely and significantly induced a cholesterol reduction comparable to that of statins (Castano et al., 1999; Crespo et al., 1999; Fernandez et al., 2001; Castano et al., 2002c; Castano et al., 2003a; Castano et al., 2003b; Gamez et al., 2003) and greater than other effective natural health products such as plant sterols (Chen et al., 2005). Complementing their lipid-lowering activity, policosanols were seen to be effective in the prevention of intermittent claudication and peripheral artery disease (PAD) through its inhibitory action on platelet aggregation (Castano et al., 1999; Castano et al., 2001a). Based on these claimed benefits, policosanols can be considered as good alternatives to statins in the treatment of dyslipidemia and the prevention of CVD.

Until 2003, all studies on policosanols originated from one single jurisdiction in Cuba and tested one specific mixture of alcohols extracted from Cuban sugar cane. 
Policosanols are found in a large variety of plants and, therefore, the need to test various sources and preparations was manifested in a series of independent studies conducted in Europe (Lin et al., 2004; Reiner et al., 2005), North America (Wang et al., 2003; Wang et al., 2005) and South Africa (Greyling et al., 2006) using extracts from wheat (Lin et al., 2004), sugar cane (Wang et al., 2003; Wang et al., 2005; Greyling et al., 2006) and rice (Reiner et al., 2005; Wang et al., 2005). None of the mentioned studies was able to replicate the substantial cholesterol lowering outcomes of original research. However, the lack of efficacy was attributed by the original laboratory group to the differing alcohol composition of policosanol mixtures in an article comparing two preparations of different minor alcohol composition (Castano et al., 2002b). In 2006, clinical trials conducted in Germany and the United States using the original SCP supported recent independent findings, reporting no reduction in blood cholesterol as a result of policosanol intake (Berthold et al., 2006; Cubeddu et al., 2006; Dulin et al., 2006). The original Cuban product has thus been reevaluated since the start of the present project. However, no independent work to date has directly compared the cholesterol-lowering effect of two different mixtures of SCP and examined their impact on the metabolism of cholesterol in vivo. Although an effect on hepatic biosynthesis of cholesterol has been suggested, other potential mechanisms have not yet been explored. Since the overall absorption of SCP is low (Menendez et al., 2005) and their effect substantial, an inhibitory action at the level of the intestine should be considered. Moreover, there is a need for independent reports to confirm the antioxidant properties of SCP on human LDL.

\subsection{Objectives}

The objectives of the present project, which includes an animal and a human component were therefore to:

1- Compare the lipid-lowering effect of the original Cuban sugar cane product (SCP1) to an alternative mixture (SCP2) in golden Syrian hamsters,

2- Assess the lipid-lowering effect of original SCP in hypercholesterolemic volunteers in a randomized double-blind placebo-controlled clinical trial, 
3- Measure cholesterol biosynthesis and intestinal absorption in hypercholesterolemic volunteers as a result of original SCP supplementation using labeled isotope methodology,

4- Measure the effect of original SCP on LDL oxidation in hypercholesterolemic volunteers using a high-precision capture ELISA procedure.

Our null hypothesis was that there would be no difference between the effects of SCP1 (Dalmer Laboratories, Cuba) and SCP2 (Degussa bioactives, Germany) on the lipid profile in hamsters. Furthermore, we hypothesized that Dalmer policosanols would have no effect on the lipid profile, LDL oxidation and cholesterol kinetics in hypercholesterolemic volunteers. 


\section{Chapter 2}

\section{Literature Review}

\subsection{Policosanols: Structure, sources and properties}

Policosanols are mixtures of very long chain fatty alcohols $\left(\left(\mathrm{CH}_{2}\right) n-\right.$ $\mathrm{CH}_{2} \mathrm{OH}$ ) with chain lengths ranging from 24 to 34 carbons. Octacosanol is the major component in most mixtures, but other alcohols including triacontanol and hexacosanol are also prevalent (Gouni-Berthold \& Berthold, 2002). Policosanols are found in a variety of plant foods including whole grains, nuts and unrefined plant oils (Hargrove et al., 2004). It is extracted from the waxy coating of stems and leaves of plants. Although extraction procedures vary (Wang et al., 2007), the patented method of extraction of SCP involves saponification and extraction with organic solvents (Patents, 1994). SCP extracts occur in the form of a crystalline light-cream powder, insoluble in water, hexane, methanol and ethanol and slightly soluble in chloroform. The purity of SCP mixtures varies but it is usually around 90\% (Mas, 2000). The alcohol composition is summarized in Table 2.1. SCP 10 mg pills were tested for stability and no significant changes in quality criteria were observed under extreme conditions such as acid base hydrolysis, oxidative and photolytic degradation (Cabrera et al., 2002). Another pharmacological study showed no interaction between the SCP active principle and excipients (Cabrera et al., 2002).

\subsection{Lipid-lowering effect of policosanols}

The effect of Cuban SCP was first reported in 1994 when the original research group found a significant effect of SCP on human lung fibroblasts (Menendez et al., 1994). Since then, a series of studies conducted by the same Cuban jurisdiction using the patented product by Dalmer Laboratories confirmed the preliminary data. These studies are outlined in Table 2.2. 


\subsubsection{Animal studies investigating policosanol efficacy}

Various animal models including rats (Menendez et al., 1996), rabbits (Arruzazabala et al., 1994; Menendez et al., 1997; Arruzazabala et al., 2000), dogs (Mesa et al., 1994) and monkeys (Mesa et al., 1994; Rodriguez-Echenique et al., 1994) were given doses ranging from $5 \mathrm{mg} / \mathrm{kg} /$ day to $500 \mathrm{mg} / \mathrm{kg} /$ day of $\mathrm{SCP}$, all of which incurred substantial reductions in total cholesterol (TC) and LDL-C levels. A 20\% reduction in TC which was recorded at week 8 was maintained throughout study periods of up to 54 weeks (Mesa et al., 1994; RodriguezEchenique et al., 1994). In the fore-mentioned study by Menendez et al. (1997), rabbits with endogenous hypercholesterolemia induced by a wheat starch casein diet were treated with SCP for a period of 30 days. Although TC and LDL-C levels had increased in the SCP group, the values were $35 \%$ and $38 \%$, respectively, lower than the control group (Menendez et al., 1994). More recently, independent research groups which have taken interest in studying policosanols failed to reproduce these results (Wang et al., 2003; Murphy et al., 2004; Wang et al., 2005). In a study conducted by Wang et al in 2003, golden Syrian hamsters were fed $25 \mathrm{mg} / \mathrm{kg}$ of SCP (Degussa Bioactives, Germany) alone and in combination with plant sterols for a period of 4 weeks. Contrary to results seen in previous SCP trials, not only did policosanol treatment not significantly affect TC and HDL-cholesterol (HDL-C), but the sugar cane extract increased non-HDL-C (Wang et al., 2003). Similarly, in 2004, Murphy et al. used $100 \mathrm{mg} / \mathrm{kg}$ of SCP on normocholesterolemic rabbits and found no significant change in serum lipids. The product Lesstanol ${ }^{\circledR}$ used in this trial is a mixture of alcohols similar in its octacosanol content (67\%) to the original Dalmer SCP (60-70\%) used in studies conducted in Cuba. However, this mixture does not contain three of the minor alcohols present in the Dalmer product. Although octacosanol is considered to be the main active compound (Mas, 2000), Lesstanol ${ }^{\circledR}$ (octa-60) was shown to have a weaker effect on serum lipids (Castano et al., 2002b). Also, the level of dietary cholesterol in the hamster study was higher compared to the previous animal trials (Menendez et al., 1996; Menendez et al., 1997). Although it was shown that the 
efficacy of drugs affecting cholesterol biosynthesis such as statins decrease serum cholesterol in a high dietary cholesterol context (Kobayashi et al., 1989), the possibility that a high cholesterol intake might suppress cholesterol synthesis and mask the effect of SCP cannot be ruled out.

\subsubsection{Human studies investigating policosanol efficacy}

Over thirty clinical trials testing the lipid-lowering potential of SCP in humans have been conducted using doses ranging from 2 to $40 \mathrm{mg} / \mathrm{day}$. The studies varied in duration and sample population, and consistently showed reductions in serum LDL-C and TC levels of up to $44 \%$ and $29 \%$, respectively (Crespo et al., 1997). Significant increases in serum HDL-C as compared to placebo were only reported in a few studies (Menendez et al., 1999b; Castano et al., 2001a; Mirkin et al., 2001) and reductions in serum triglycerides in only two (Castano et al., 2002c; Mas et al., 2004).The lipid-lowering potential of SCP tended to increase in a dose- dependant manner up to $20 \mathrm{mg} / \mathrm{day}$. Beyond this dose, no further cholesterol reductions were observed, as reported in 2001 by Castano et al in a randomized double-blind placebo-controlled study where the administration of $20 \mathrm{mg}$ versus $40 \mathrm{mg}$ of policosanols resulted in similar reductions in TC and LDL-C (Castano et al., 2001b). Human trials designed with the aim of determining the long term effects of SCP demonstrated that cholesterol reductions were maintained throughout the study period (Pons et al., 1994b; Canetti et al., 1995b; Batista et al., 1996). The smallest dose administered to volunteers was used by Batista et al in a clinical trial where $1 \mathrm{mg}$ of SCP taken twice daily by hypercholesterolemic patients for a period of 14 months resulted in reductions of $15.6 \%$ and $14.8 \%$ in TC and LDL-C levels, respectively.

The sample populations involved in SCP human trials included different age groups and health statuses: healthy volunteers (Menendez et al., 2000a), hypercholesterolemic adolescents (Castaño et al., 2002) and adults (Castano et al., 2001b), post-menopausal women (Mirkin et al., 2001) and cardiac elderly patients (Mas et al., 2001). In a randomized double-blind clinical-trial in older hypercholesterolemic patients with coronary heart disease (Mas et al., 2001), 280 
60-80 year old men and women received $5 \mathrm{mg}$ of SCP or matching controls to consume with the evening meal for 1 year. Volunteers were seen at baseline and after 3, 6, 9 and 12 months of therapy. At the end of the study, LDL-C and TC levels dropped by $21.3 \%$ and $15.9 \%$, respectively. HDL-C levels were increased by $26.1 \%$ and even triglycerides were decreased by $7.8 \%$. In terms of serious adverse events, 1 policosanol recipient versus 10 placebo recipients experienced coronary events.

SCP are considered to be good alternatives to statin drugs because of the similar mechanism of action characterizing both lipid-lowering agents (GouniBerthold \& Berthold, 2002). In fact, several human clinical trials were conducted by the original research group in Cuba, aiming to establish a direct comparison between policosanols and other lipid-lowering drugs, namely HMG-CoAreductase inhibitors or statins (Varady et al., 2003). In 2001, Fernandez et al studied the efficacy of $10 \mathrm{mg} /$ day of policosanols versus $20 \mathrm{mg} /$ day of fluvastatin in elderly hypercholesterolemic women (Fernandez et al., 2001). The study was randomized and single-blinded but was not placebo-controlled. Results showed significant decreases in LDL-C levels of $29.2 \%$ and $22.9 \%$ and reductions in TC of $19.3 \%$ and $16.7 \%$ for SCP and fluvastatin treatments, respectively. SCP was compared to lovastatin in three randomized double-blind non-placebo-controlled studies (Crespo et al., 1999; Castano et al., 2002c; Castano et al., 2003b), showing similar efficacy for both treatments on TC and LDL-C and a greater improvement of HDL-C as a result of SCP intake. However, Atorvastatin, the most efficaceaous statin drug resulted in a more pronounced decrease in LDL-C and TC (42\% and $32 \%)$ than SCP (26\% and 18\%) in studies targeting elderly patients with type 2 hypercholesterolemia (Castano et al., 2003b) and patients with dyslipidemia associated with type 2 diabetes mellitus (Castano et al., 2003a). $\mathrm{SCP}$ research conducted in Cuba has shown promising results in terms of cholesterol-lowering and CVD prevention. However, common limitations to these studies were poor monitoring of subjects lifestyle habits and broad selection criteria. In fact, volunteer visits were not frequent, ranging from once every four weeks (Castano et al., 2003b) to once every three months (Mas et al., 2001). 
During visits, SCP tablets were counted as a measure of compliance. The clinical staff gave participants dietary advice to follow the NCEP Step I diet with little or no follow up thereafter. As for selection criteria, studies targeting hypercholesterolemic individuals included subjects with BMIs greater than 30 $\mathrm{kg} / \mathrm{m}^{2}$, type 2 diabetics (Mas et al., 2001), and in some instances, no washout period was required prior to the study from subjects taking lipid-lowering drugs (Mirkin et al., 2001). Knowing that obesity, diabetes and/or the intake of lipidlowering drugs affect cholesterol metabolism, including such variables in the study population could give rise to non-responders or over-responders which would potentially confound the effect of SCP treatments.

More recently a growing body of evidence has emerged from independent laboratories testing the effects of policosanols on hypercholesterolemic individuals (Lin et al., 2004; Reiner et al., 2005; Berthold et al., 2006; Cubeddu et al., 2006; Dulin et al., 2006; Greyling et al., 2006). Various sources of policosanols such as wheat germ (Lin et al., 2004), rice (Reiner et al., 2005) and sugar cane, both the original mixture (Berthold et al., 2006; Dulin et al., 2006) and alternative preparations (Cubeddu et al., 2006; Greyling et al., 2006) have been shown to have no significant cholesterol-lowering effect. Inclusion criteria for study populations were similar to the Cuban original studies, so was the case for policosanol dose and administration regimen. The matrix used in the administration was usually the tablet on its own, with the exception of one study which incorporated policosanols into chocolate pellets (Lin et al., 2004). All studies supplemented the treatment or placebo with the evening meal as per previous research, based on the fact that cholesterol synthesis is highest at night (Parker et al., 1982; Cella et al., 1995). In addition, the subjects' diets were not controlled with the exception of dietary advice to follow an NCEP Step I diet, as was done previously in Cuban research. In addition, tablet counting at different time points during the study was used to verify compliance. The lack of efficacy observed in these trials remains inconclusive due to the variability in the policosanol source and composition. Although a thorough alcohol composition analysis was conducted in most of the mentioned trials to verify that the mixture 
used was very similar to the original product, it remains that only two studies (Berthold et al., 2006; Dulin et al., 2006) used the original SCP extract provided by Dalmer Laboratories. The study from Berthold et al was a multicenter, randomized, double-blind placebo-controlled trial where 143 hypercholesterolemic patients were randomized to control or 10, 20, 40 and 80 $\mathrm{mg}$ /day of SCP. The authors reported no significant difference among the five parallel arms of the trial.

The evidence from independent studies tends to refute claims made by the Cuban research on SCP efficacy. However, there is a need for more clinical studies evaluating SCP in a context providing better ensured compliance and using the original SCP product.

\subsection{Effect of policosanols on cholesterol metabolism.}

Studies investigating responses of cholesterol metabolism in relation to SCP supplementation have established that the resulting cholesterol-lowering effect, mainly mediated by a decrease in serum LDL-C levels, was associated with an inhibition of hepatic cholesterol synthesis (Menendez et al., 1994; Menendez et al., 1997; Menendez et al., 2001a) and the stimulation of receptormediated LDL uptake by the liver (Menendez et al., 1996; Menendez et al., 1997). Cholesterol synthesis was assessed in vitro by measuring the enrichment of tritiated water in human fibroblast (Menendez et al., 2001a) and animals (Menendez et al., 1997) and was seen to decrease as a result of SCP treatment. Unlike statins which are known to inhibit cholesterol biosynthesis by affecting the rate-limiting enzyme HMG-CoA reductase (Stancu \& Sima, 2001), SCP were shown to possess a different, more complex, mechanism of action. In studies on human cultured fibroblasts (Menendez et al., 1994; Menendez et al., 2001a), ${ }^{14} \mathrm{C}$ acetate and ${ }^{14} \mathrm{C}$ mevalonate were added to cells pre-treated with $0.5,5$ and 50 $\mu \mathrm{g} / \mathrm{ml}$ of policosanols in order to determine the incorporation of radioactivity from both precursors into free cholesterol as compared to non- treated control cells. The authors observed that as policosanol concentration increased, radioactivity incorporated from acetate, but not from mevalonate, decreased 
significantly, reaching an inhibition of $49.7 \%$ at the $50 \mu \mathrm{g} / \mathrm{ml}$ dose. It was therefore concluded that SCP interferes in the cholesterol biosynthetic pathway at a step between acetate and mevalonate production.

The direct effect of SCP on HMG-CoA reductase was tested by the addition of ${ }^{14} \mathrm{C}$-HMG-CoA to cultured renal fibroblasts pre-treated with $0.5,5$ and $50 \mu \mathrm{g} / \mathrm{ml}$ of policosanols (Menendez et al., 1994; Menendez et al., 2001a). Mevalonate production was measured as a marker of HMG-CoA reductase activity and results showed no significant difference in mevalonate concentration for any of the three doses of SCP as compared to control, supporting the hypothesis that the effect on cholesterol synthesis occurs at a step between acetate incorporation and mevalonate production. Since decreased cholesterol biosynthesis is associated with increased LDL-receptor expression in hepatocytes (Basu et al., 1976; Hobbs et al., 1992), the effect of SCP on serum LDL-C can be partly explained by increased LDL- binding, uptake and degradation as demonstrated in studies measuring radio-labeled LDL on the surface of human fibroblasts (Menendez et al., 1994).

Recently, original findings were replicated by an independent laboratory in Kentucky (Singh et al., 2006) using methods developed by Cuban researchers. Policosanols and individual alcohols were tested in rat hepatoma cells and the incorporation of ${ }^{14} \mathrm{C}$-acetate and ${ }^{14} \mathrm{C}$-mevalonate measured. The authors demonstrated a dose-dependant decrease in cholesterol labeling reaching 30\% at $25 \mu \mathrm{g} / \mathrm{ml}$ of SCP. A similar reduction was seen in the case of triacontanol (C30), whereas it was less pronounced for hexacosanol (C26), heptacosanol (C27) and octacosanol (C28). When ${ }^{14} \mathrm{C}$-mevalonate was added to the medium, no change in cholesterol labeling was recorded for policosanols and other individual alcohols. However, when the dose of SCP was doubled, a slight decrease in the rate of incorporation of mevalonate was seen. The direct effect of SCP on HMG-CoA reductase was tested as well, showing no change in activity in response to SCP (Singh et al., 2006). Both original and independent in vitro isotope studies are crucial to the understanding of the mechanism of policosanols mainly because they determine that SCP affects biosynthesis at a level between acetate and 
mevalonate production without altering HMG-CoA reductase activity (Figure 2.1). An alternative mechanism involving an up-regulation of AMP kinase, associated with decreased cholesterol synthesis was suggested as a result of an observed increased concentration of the enzyme after SCP treatment. In addition, findings reinforce the importance of the mixture composition since different alcohols yielded variable outcomes (Singh et al., 2006).

However, the experimental method can be strongly criticized in these studies because of its application to metabolically active compounds such as policosanols. Since policosanols undergo beta-oxidation in the cell, it might contribute metabolites such as acetate to the medium, therefore diluting the amount of labeled ${ }^{14} \mathrm{C}$-acetate incorporated in cholesterol. The incorporation of ${ }^{14} \mathrm{C}$-mevalonate decreased only at a higher dose which could be explained by the preferential incorporation of ${ }^{14} \mathrm{C}$-mevalonate into cholesterol at a low dose of SCP. At a higher concentration of policosanols, a dilution effect might be exerted when more non-labeled acetate is formed in the medium and used to make mevalonate for incorporation into cholesterol. The alternative mechanism suggested by the authors involving AMP-kinase is a plausible hypothesis which has to be confirmed since this enzyme is involved in other pathways such as beta oxidation (Hardie \& Carling, 1997); therefore an increase in its activity could only be a reflection of a higher rate of beta oxidation due in part to the addition of policosanols.

Changes in cholesterol biosynthesis in response to policosanol feeding were measured by Wang et al $(2003,2005)$ in vivo using the hamster model. Contrary to previous research, the authors used a single stable isotope ratio method, measuring the incorporation of deuterium into de novo cholesterol (Wang et al., 2003; Wang et al., 2005). This method has been validated against the more commonly used dual stable isotope ratio method and shown to provide a reliable measurement of cholesterol biosynthesis (Wang et al., 2004). Policosanol supplementation to hamsters in the aforementioned studies by Wang et al (2003, 2005) showed no effect on cholesterol biosynthesis, therefore, contributing to the controversy associated with policosanols. This leads us to acknowledge that the 
lipid-lowering mechanism of SCP is not fully understood, especially since other cholesterol-lowering mechanisms such as inhibition of cholesterol or bile acid intestinal absorption have not been investigated.

\subsection{Effect of sugar cane policosanols on lipid peroxidation.}

Many processes have been implicated in early atherogenesis and competing theories on the key events triggering atherosclerosis include lipoprotein oxidation, lipoprotein aggregation, macrophage chemotaxis and foam cell formation (Figure 2.2). In a meta-analysis conducted by Williams and Tabas in 1995 (Williams \& Tabas, 1995), the authors suggest that atherogenesis is triggered by the stimulation of intramural synthesis of molecules, such as proteoglycans, which promote lipoprotein retention, and that trapped lipoproteins are then subjected to oxidative stress proposed to be the second most central process in atherogenesis (Williams \& Tabas, 1995). Oxidized LDL particles are then taken up by macrophages, leading to cholesterol accumulation and foam cell formation within the artery (Aviram, 1993). Prevention of LDL oxidation was shown to be one of SCP cardioprotective properties. Animal (Menendez et al., 1999b; Menendez et al., 2000b) and human studies (Menendez et al., 2000b; Castano et al., 2002c) show decreased lipid peroxidation as a result of SCP treatment. In rabbits with exogenous hypercholesterolemia, policosanols had a dose-dependant protective effect on atherosclerotic lesions, as 25 and 200 $\mathrm{mg} / \mathrm{kg} /$ day of SCP resulted in significantly less aortic fatty streaks with foam cells of macrophage origin (Arruzazabala et al., 1994). In a study conducted by Menendez et al.(1999), 0, 250 and $500 \mathrm{mg} / \mathrm{kg} /$ day were administered to rats over a period of 4 weeks. $\mathrm{CuSO}_{4}$ oxidation was initiated in LDL isolated from fasted blood plasma. Lipid oxidation was evaluated by continuous monitoring of conjugated dienes and measuring thiobarbituric acid reactive substances (TBARS). SCP significantly prolonged the lag time, reduced the propagation of conjugated diene generation and decreased levels of TBARS (Menendez et al., 1999b). A similar experiment was conducted in healthy volunteers given $5 \mathrm{mg}$ and $10 \mathrm{mg}$ of SCP for a period of 8 weeks (Menendez et al., 2000a). SCP 
significantly decreased the rate of conjugated diene generation by $38 \%$ and lowered malonaldehyde (MDA) generation by $32 \%$. SCP was also compared to lovastatin in terms of antioxidant properties in a randomized double-blind study (Castano et al., 2002c). Both substances decreased conjugated diene and TBARS production but only SCP increased the lag time of LDL peroxidation and total plasma antioxidant activity. Since LDL oxidation is considered to be a crucial step in the development of atherosclerosis, the antioxidant advantage of policosanols makes them good alternatives to statins.

Antioxidant properties of SCP were studied by one independent research group in a trial conducted in 2005 disproving the antioxidant properties of policosanols (Ng et al., 2005). The authors measured anti-LDL oxidation activity as well as the anti free-radical scavenging potential of SCP as a result of incubating human LDL in tetracosanol, hexacosanol and octacosanol. Oxidative damage was measured by monitoring TBARS and anti-free radical scavenging capacity was determined by measuring the scavenging of 2,2-diphenyl-1-picrylhydrazyl. Results from this trial show no significant difference in antioxidant activity of different alcohols as compared to control. There are no independent studies to date that examined the antioxidant effect of SCP in a human population. Moreover, methods used to assess oxidative damage as a result of SCP supplementation should be more precise and specific to LDL oxidation. Although TBARS is widely used for its simplicity, it is known to be affected by reaction conditions, namely the presence of EDTA (Lapenna et al., 2001). In a comparative study conducted by Carru et al in 2004, the authors reported that copper oxidation was not a suitable method for the clinical evaluation of LDL- oxidation because of the difficulty in standardizing the test and the time needed to run it. More importantly, it did not provide a reliable measurement of conjugated dienes (Carru et al., 2004). LDL oxidation can be precisely determined by using an enzyme immunoassay based on a direct sandwich technique using murine monoclonal antibodies targeting two different antigenic determinants of the oxidized LDL (Mercodia, Sweden). Oxidized LDL was first correlated with coronary artery disease using this approach in two studies conducted by Holvoet et al (Holvoet et al., 1998; Holvoet 
et al., 2001) who established a strong relationship between the level of oxidized LDL in the blood and coronary artery disease. Several studies attest to the importance of oxidized LDL as a marker of cardiovascular disease risk (Hulthe \& Fagerberg, 2002; Kopprash et al., 2002; Sigurdardottir et al., 2002). Moreover, the ELISA immunoassay has proved to be a reliable, precise method to measure levels of oxidized LDL in plasma samples stored for up to nine years (Holvoet et al., 2001; Hulthe \& Fagerberg, 2002). Therefore, independent studies examining the effect of SCP in humans using reliable methods such as the sandwich ELISA, are needed in the controversial field of policosanol research.

\subsection{Composition and metabolism of policosanol mixtures}

The source and composition of policosanol mixtures are now at the core of the controversy around their cholesterol-lowering efficacy, especially since the use of different policosanol products resulted in different research outcomes (Varady et al., 2003; Wang et al., 2003; Lin et al., 2004; Reiner et al., 2005; Wang et al., 2005; Greyling et al., 2006). Although compositions of different mixtures have not been directly compared to date, chemical analyses of certain alternative policosanol preparations (Wang et al., 2003; Lin et al., 2004; Wang et al., 2005) show very little difference with the original Cuban SCP, especially in their octacosanol content, deemed to be the active component of the mixture. Similarly, little is known on the exact metabolism of policosanols. In a study conducted by the original Cuban research group on the metabolism of octacosanol, the authors found the intestinal absorption of the alcohol to be very poor (Menendez et al., 2005). However, policosanols are suggested to affect the activity of HMG-CoA reductase in the peroxisomes. Knowing that SCP is effective at doses as low as $2 \mathrm{mg}$ /day (Batista et al., 1996), this raises the interest in other potential mechanisms that would allow substantial reductions with such low absorption rates. As mentioned earlier, the effect of SCP on cholesterol intestinal absorption needs to be explored. Moreover, evidence which is available on the metabolism of SCP mainly includes studies using radiolabelled policosanols (Menendez et al., 1994; Menendez et al., 2001a) in order to trace the 
signal in the different tissues and feces. Since detecting the label does not discriminate between the molecule itself and its metabolites, there is a need for further research to examine the exact metabolism of SCP.

\subsection{Safety and tolerability of sugar cane policosanols}

Animal studies of varying durations showed that SCP is safe up to a dose of $5,000 \mathrm{mg} / \mathrm{kg} / \mathrm{day}$, which is more than 17000 times the human daily dose ranging from $5 \mathrm{mg}-20 \mathrm{mg} /$ day. In 6-12 months long toxicity studies conducted on Sprague-Dawley rats (Aleman, 1994b; Gamez et al., 2001), toxicity related to the administration of 50, 500, 2500 and $5000 \mathrm{mg} / \mathrm{kg}$ /day of SCP through gavage was investigated. Results showed no difference from control in terms of blood biochemistry, hematological and anatopathophysiological parameters. Longer term studies on dogs (Mesa et al., 1994) using doses equivalent to 620 times the therapeutic dose reported no adverse effect throughout a 24-month period. Carcinogenicity of SCP has been investigated in rats and no difference was observed between the treatment group and the control with respect to occurrence of benign and malignant tumors after a 24-month supplementation period (Aleman, 1994a).

While statins have been shown to induce a slight increase in hepatic enzymes in humans (Castano et al., 2002c; Castano et al., 2003b), SCP mixtures did not affect these parameters and were even seen to play a protective role against liver (Noa et al., 2003) and endothelial (Noa et al., 1997) damage, atherosclerotic lesions (Noa et al., 1995) as well as myocardial necrosis, delaying the evolution of infarction in rats. Rodriguez et al tested peri- and post-natal toxicity and teratogenicity of policosanols in rats and rabbits in studies involving doses of 5, 50, 500 and $1000 \mathrm{mg} / \mathrm{kg} /$ day of policosanols during pregnancy and after parturition. No signs of toxic effects in the mothers, and embryotoxic, teratogenic or fetotoxic effects in the offspring were observed (Rodriguez \& Garcia, 1994; Rodriguez \& Garcia, 1998). In a multi-generation reproduction study from the same authors, male and female rats were given 0, 5, 50 and 500 $\mathrm{mg} / \mathrm{kg} /$ day of SCP. No deleterious effects on fertility, reproductive performance 
or development of pups from three successive generations were observed as a result of any of the three doses administered as compared to control (Rodriguez et al., 1997). In humans, policosanols effectively reduced serum LDL-C with no associated serious side effects and reported mild side effects were more common in the placebo group than in the policosanol group (Castano et al., 2001a; Mas et al., 2001; Mirkin et al., 2001). In studies comparing effects of SCP and statin drugs on the lipid profile (Fernandez et al., 2001; Castano et al., 2003a) statins significantly increased levels of alanine aminotransferase (ALT), aspartate aminostransferase (AST) and creatinine phosphokinase (CPK). On the other hand, SCP did not have any effect on liver enzymes. The only two side effects considered to be possibly related to SCP were dizziness and acidity (Fernandez et al., 2001).

Interaction of SCP with concomitant medication was tested with warfarin and $\beta$-blockers in rats. SCP were not seen to influence the effect of warfarin on bleeding time (Carbajal et al., 1998) but seemed to increase the hypotensive effect of $\beta$-blockers without modifying cardiac frequency (Molina et al., 1998). Although no drug interaction assessments have been conducted on humans to date, SCP can be considered as a safe supplement within the prescribed dosages.

\subsection{Summary and conclusions}

Sugar cane policosanols have shown to improve the lipid profile substantially over the past decade in a series of studies conducted by a single jurisdiction in Cuba. However, recent findings emanating from independent research groups have raised doubt on the efficacy of these natural health products both in animals and humans. Although SCP was shown to inhibit cholesterol biosynthesis by indirectly affecting the HMG-CoA activity, its absorption was shown to be very limited. Therefore, policosanol metabolism and its interaction with cholesterol remain unclear. The alcohol composition of the original Cuban SCP is thought to be at the origin of the substantial reductions in LDL-C seen in the extensive Cuban literature on policosanols. Alternative policosanol extracts were reported to possess alcohol compositions similar to the original mixtures, 
however, no independent evidence exists that shows substantial reductions as a result of supplementation with those mixtures. Composition and metabolism are two of the numerous gaps in policosanol research which need to be filled in order to solve the controversy around its efficacy. Others include the effect of policosanols on cholesterol metabolism as well as its effect on LDL-oxidation which has only been recorded in studies conducted by the original Cuban researchers and refuted by one independent animal study.

Policosanols extracted from sugar cane, wheat, rice and beeswax have become very popular over-the-counter supplements, prescribed by doctors as therapy or prevention against cardiovascular diseases. Furthermore, major pharmaceutical companies have adopted policosanols as a means to add cholesterol-lowering value to their supplements (e.g. one-a-day cholesterol plus from Bayer). Since research on policosanols remains inconclusive to this date, there is a need to evaluate the cholesterol-lowering potential of both the original and alternative mixtures of policosanols as well as to determine their exact mechanism of action in animals and humans, to ensure that the use of policosanols as a means to decrease the risk of cardiovascular incidents is safe and effective. 
Chapter 2: Literature review

Table 2.1. Composition of original sugar cane policosanol extract

\begin{tabular}{lcc}
\hline \multicolumn{1}{c}{ Alcohol } & Structure & Percent (\%) \\
\hline Tetracosanol & $\mathrm{C}_{24} \mathrm{H}_{49} \mathrm{OH}$ & $<2$ \\
Hexacosanol & $\mathrm{C}_{26} \mathrm{H}_{53} \mathrm{OH}$ & $4.5-10$ \\
Heptacosanol & $\mathrm{C}_{27} \mathrm{H}_{55} \mathrm{OH}$ & $<5$ \\
Octacosanol & $\mathrm{C}_{28} \mathrm{H}_{57} \mathrm{OH}$ & $60-70$ \\
Nonacosanol & $\mathrm{C}_{29} \mathrm{H}_{59} \mathrm{OH}$ & $<2$ \\
Triacontanol & $\mathrm{C}_{30} \mathrm{H}_{61} \mathrm{OH}$ & $10-15$ \\
Dotriacontanol & $\mathrm{C}_{32} \mathrm{H}_{65} \mathrm{OH}$ & $3-8$ \\
Tetriacontanol & $\mathrm{C}_{34} \mathrm{H}_{69} \mathrm{OH}$ & $<2$ \\
\hline
\end{tabular}

Octacosanol is the major alcohol in policosanol mixtures and is considered to be the active component of the lipid-lowering agent.

(Mas, 2000) 
Table 2.2. Recent policosanol supplementation studies conducted by original (1) and independent (2) research groups

\begin{tabular}{|c|c|c|c|c|c|c|}
\hline Reference & $\begin{array}{l}\text { Subjects } \\
\text { (number) }\end{array}$ & $\begin{array}{l}\text { Policosanol } \\
\text { source }\end{array}$ & $\begin{array}{l}\text { Dosage } \\
(\mathrm{mg} / \mathrm{d})\end{array}$ & \multicolumn{3}{|c|}{$\%$ change in cholesterol levels } \\
\hline 1- Studies show & ng cholesterc & efficacy & & Total & LDL & HDL \\
\hline $\begin{array}{c}\text { (Castano, Mas et al, } \\
2000)\end{array}$ & $\begin{array}{l}\text { PMW, HC } \\
\quad(244)\end{array}$ & CSCP & $5 ; 10 \mathrm{wks}$ & -12.6 & -17.7 & +16.7 \\
\hline $\begin{array}{c}\text { (Menendez, Mas et } \\
a l, 2000)\end{array}$ & $\mathrm{NC}(69)$ & CSCP & $5 ; 8$ wks & -10.5 & -16.7 & +9.0 \\
\hline $\begin{array}{c}\text { (Castano, Mas et } \\
a l, 2001)\end{array}$ & $\mathrm{HC}(89)$ & CSCP & $20 ; 24$ wks & -15.6 & -27.4 & +17.6 \\
\hline $\begin{array}{c}\text { (Mirkin, Mas et al, } \\
\text { 2001) }\end{array}$ & $\begin{array}{l}\text { PMW, HC } \\
(56)\end{array}$ & CSCP & $5 ; 8$ wks & -12.9 & -17.3 & $\mathrm{n} / \mathrm{c}$ \\
\hline $\begin{array}{c}\text { (Castano, } \\
\text { Fernandez et al, } \\
\text { 2002) }\end{array}$ & $\mathrm{A}, \mathrm{HC}(55)$ & $\mathrm{CSCP}$ & $5 ; 12$ wks & -21.9 & -32.6 & +10.1 \\
\hline $\begin{array}{c}\text { (Castano, Mas et al, } \\
\text { 2003) }\end{array}$ & $\mathrm{E}, \mathrm{HC}(75)$ & CSCP & $10 ; 8$ wks & -16.4 & -23.1 & +5.3 \\
\hline $\begin{array}{c}\text { (Castano, Mas et al, } \\
\text { 2003) }\end{array}$ & $\mathrm{HC}(28)$ & CSCP & $10 ; 20$ wks & -17.5 & -31.0 & +31.5 \\
\hline $\begin{array}{c}\text { (Mas, Castano et al, } \\
\text { 2004) }\end{array}$ & $\begin{array}{c}\mathrm{O}, \mathrm{HC} \\
(129)\end{array}$ & CSCP & $10 ; 3$ yrs & -20.1 & -31.8 & +24.6 \\
\hline $\begin{array}{c}\text { (Mas, Castano et al, } \\
\text { 2004) }\end{array}$ & $\begin{array}{l}\text { DM, HC } \\
(239)\end{array}$ & CSCP & $10 ; 2$ yrs & -21.9 & -29.5 & +12.4 \\
\hline
\end{tabular}




\begin{tabular}{|c|c|c|c|c|c|c|}
\hline Reference & $\begin{array}{l}\text { Subjects } \\
\text { (number) }\end{array}$ & $\begin{array}{l}\text { Policosanol } \\
\text { source }\end{array}$ & $\begin{array}{l}\text { Dosage } \\
(\mathrm{mg} / \mathrm{d})\end{array}$ & \multicolumn{3}{|c|}{$\%$ change in cholesterol levels } \\
\hline \multicolumn{4}{|c|}{ 2. Studies Failing to Show Cholesterol-lowering Efficacy } & $\mathrm{TC}$ & LDL & HDL \\
\hline Lin et al, 2004 & $\begin{array}{c}\mathrm{NC} \text {-mild } \\
\mathrm{HC}(58)\end{array}$ & WGP & $20 ; 4$ wks & $\mathrm{n} / \mathrm{c}$ & $\mathrm{n} / \mathrm{c}$ & $\mathrm{n} / \mathrm{c}$ \\
\hline \multirow{2}{*}{$\begin{array}{c}\text { Reiner et al, } 2005 \\
\text { Greyling et al, } \\
2006\end{array}$} & $\mathrm{HC}(70)$ & $\mathrm{RPC}$ & $10 ; 8$ wks & -0.05 & $\mathrm{n} / \mathrm{c}$ & $\mathrm{n} / \mathrm{c}$ \\
\hline & $\mathrm{HC}(44)$ & SCP & $20 ; 12 \mathrm{wks}$ & $\mathrm{n} / \mathrm{c}$ & $\mathrm{n} / \mathrm{c}$ & $\mathrm{n} / \mathrm{c}$ \\
\hline $\begin{array}{c}\text { Berthold et al, } \\
2006\end{array}$ & $\begin{array}{c}\mathrm{HC} \text { or HL } \\
\quad(143)\end{array}$ & $\mathrm{CSCP}$ & $80 ; 12$ wks & $\mathrm{n} / \mathrm{c}$ & $\mathrm{n} / \mathrm{c}$ & $\mathrm{n} / \mathrm{c}$ \\
\hline $\begin{array}{c}\text { Cubeddu et al, } \\
2006\end{array}$ & $\mathrm{HC}(99)$ & $\mathrm{SCP}$ & $20,12 \mathrm{wks}$ & $\mathrm{n} / \mathrm{c}$ & $\mathrm{n} / \mathrm{c}$ & $\mathrm{n} / \mathrm{c}$ \\
\hline Dulin et al, 2006 & $\begin{array}{l}\text { Mild HC } \\
\text { (40) }\end{array}$ & SCP & 8 wks & $\mathrm{n} / \mathrm{c}$ & $\mathrm{n} / \mathrm{c}$ & $\mathrm{n} / \mathrm{c}$ \\
\hline
\end{tabular}

$\mathrm{HC}=$ type II Hypercholesterolemic, $\mathrm{NC}=$ normocholesterolemic, $\mathrm{HL}=$ hyperlipidemia, $\mathrm{ARF}=$ atherosclerotic risk factor, DM = type 2 diabetes, $\mathrm{PMW}=$ postmenopausal women, $\mathrm{E}=$ elderly ( $>65$ years), $\mathrm{A}=$ adolescents $(11-19$ years $), \mathrm{O}=$ obese $(\mathrm{BMI} \geq 30), \mathrm{WGP}=$ wheat germ policosanols, $\mathrm{RPC}=$ rice policosanols, $\mathrm{SCP}=$ sugarcane policosanols, $\mathrm{CSCP}=\mathrm{Cuban}$ sugarcane policosanols, $\mathrm{n} / \mathrm{c}=$ no change 
Figure 2.1. Effect of sugar cane policosanols (SCP) on hepatic cholesterol biosynthesis

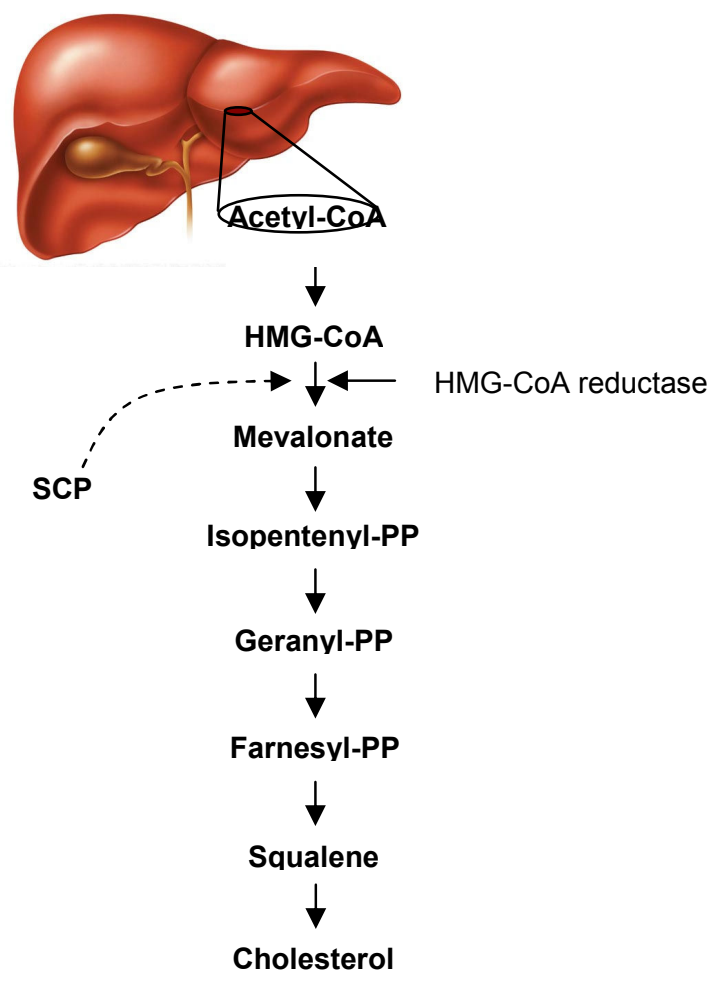

Figure 2.1.; Sugar cane policosanol reduces cholesterol synthesis by indirectly affecting the activity of HMG-CoA at a level between acetate and mevalonate production 
Figure 2.2. Reduction of LDL-cholesterol oxidation as a result of SCP supplementation

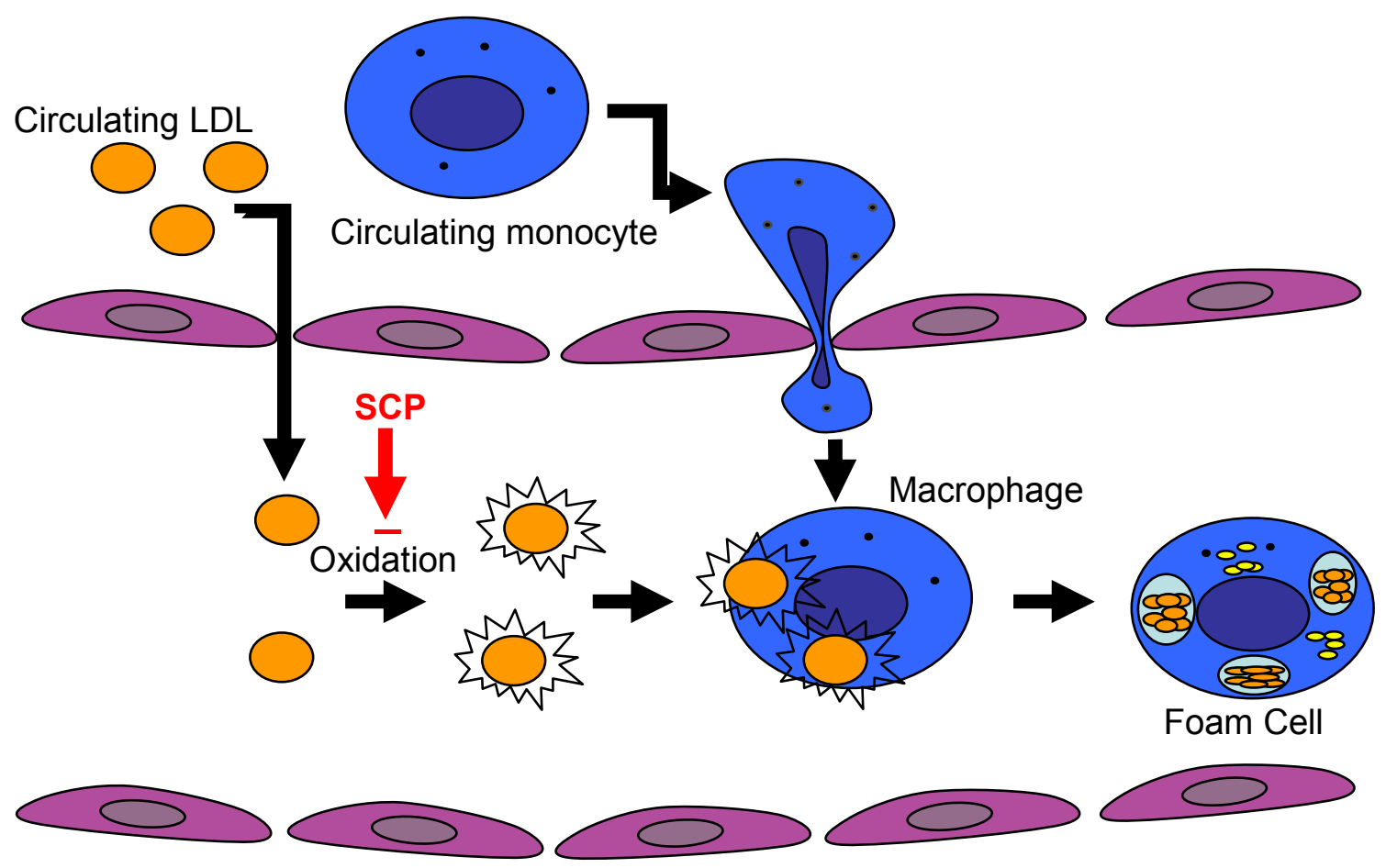




\section{Chapter 3}

\section{Manuscript 1: Lack of effect of sugar cane policosanols on plasma cholesterol in golden Syrian hamsters}

Amira N. Kassis, Christopher P.F. Marinangeli, Deepak Jain, Naoyuki Ebine and Peter J.H. Jones

\section{Author affiliation:}

School of dietetics and human nutrition, McGill University, Ste-Anne-de-Bellevue, Montréal, Quebec, H9X 3V9, Canada.

This manuscript has been published in press in Atherosclerosis

Kassis AN, Marinangeli CP, Jain D, Ebine N, Jones PJ. Lack of effect of sugar cane policosanol on plasma cholesterol in golden Syrian hamsters. Atherosclerosis. 2007;194:153-8

This project was funded by the Advanced Foods and Materials Network- Network of Centers of Excellence 
Chapter 3: Manuscript 1

\section{Manuscript outline}

3.1. Abstract

3.2. Introduction

3.3. Materials and methods

3.3.1. Animals and diets

3.3.2. Protocol

3.3.3. Plasma lipid analysis

3.3.4. Statistical analysis

3.4. Results

3.4.1. Growth of hamsters across the study period

3.4.2. Food intake of hamsters throughout the feeding period

3.4.3. Growth and intake of hamsters versus plasma lipids

3.4.4. Lipid values as a result of policosanol feeding

3.5. Discussion

3.6. Conclusion

3.7. Acknowledgments 


\subsection{Abstract}

Background: Policosanols are mixtures of higher aliphatic alcohols shown to have beneficial effects on plasma lipid levels in animals and humans. Over 50 studies have reported significant reductions in plasma cholesterol using policosanols obtained from Cuban sugar cane (Dalmer, La Havana, Cuba). However, other research groups using policosanols from alternative sources have failed to reproduce the efficacy of these alcohols observed in earlier studies.

Objectives: Therefore, the objective of the present study was to compare the cholesterol-lowering effect of the Dalmer sugar cane policosanol product versus an alternative mixture of similar policosanol composition.

Methods: Forty-eight male Golden Syrian hamsters were randomly assigned to 4 groups and fed experimental diets ad libitum for a period of 4 weeks: (i) non-cholesterol control, (ii) $0.1 \%$ cholesterol control, (iii) $0.1 \%$ cholesterol diet supplemented with $275 \mathrm{mg} / \mathrm{kg}$ diet of Dalmer Cuban sugar cane policosanols and (iv) $0.1 \%$ cholesterol diet supplemented with $275 \mathrm{mg} / \mathrm{kg}$ diet of alternative sugar cane policosanols. Hamsters were sacrificed and blood was collected at the end of the feeding period.

Results: Body weights and food intakes were similar across study groups. Neither of the two policosanol treatments had any significant effect on plasma lipid levels, as compared to cholesterol control.

Conclusion: The outcome of the present study questions the clinical usefulness of policosanol mixtures as cholesterol-lowering nutraceuticals. 


\subsection{Introduction}

Sugar cane policosanols (SCP) is a mixture of higher aliphatic alcohols with chain lengths ranging from 24 to 34 carbons, the most important and prevalent of which is octacosanol (C28). Cuban SCP, derived from the waxy coating of stems and leaves of sugar cane and other plant materials, has been shown to exert beneficial effects on lipid profiles in animals and humans in numerous studies conducted by a single laboratory and using an SCP product manufactured by Dalmer laboratories (La Havana, Cuba) (Mas, 2000). Animal trials testing the alcohols within experimental models including rats, rabbits, dogs and monkeys, have demonstrated reductions in circulating total cholesterol (TC) levels consistently greater than 13\% (Arruzazabala et al., 1994; Mesa et al., 1994; Rodriguez-Echenique et al., 1994; Menendez et al., 1996; Menendez et al., 1997). Similarly, human policosanol feeding trials (Menendez et al., 2000c; Castano et al., 2001b; Mirkin et al., 2001; Castaño et al., 2002) have shown reductions of up to $20 \%$ and $30 \%$ in TC and low-density-lipoprotein cholesterol (LDL-C) levels respectively, depending on dose and duration of SCP treatment. However, laboratories external to the original Cuban research group have failed to reproduce the results of original research in studies using hamsters (Wang et al., 2003), rabbits (Murphy et al., 2004), as well as in human clinical trials on hypercholesterolemic volunteers (Lin et al., 2004; Reiner et al., 2005). Despite similar octacosanol content, potential explanations for the disparity in results of studies conducted outside of Cuba may lie in the fact that the policosanol mixtures used in these trials differed in minor alcohol content (Wang et al., 2003; Lin et al., 2004; Murphy et al., 2004; Reiner et al., 2005; Greyling et al., 2006). To date, independent laboratories have only conducted two human studies examining the effects of the Dalmer policosanol mixture on the lipid profile (Berthold et al., 2006; Kassis \& Jones, 2006). No significant changes in TC and LDL-C levels were observed. Therefore, the similarity of the composition of policosanol mixtures to the Dalmer mixture shown to reduce blood cholesterol remains at the core of the controversy regarding policosanol lipid-lowering action.

The objective of the present study was to compare the lipid-lowering effect of the Dalmer Cuban product (SCP1) and an alternative mixture (SCP2) in the golden Syrian hamster, one used routinely in studies of dietary lipid effects. It was hypothesized that there would be no difference between the effect of SCP1 and SCP2 on plasma lipids. 


\subsection{Materials and methods}

\subsubsection{Animals and diets}

Two-week old male golden Syrian hamsters $(n=48)$ weighing 80 to $120 \mathrm{~g}$ were housed in individual cages with free access to water and were subjected to a $12 \mathrm{hr}$ light/dark cycle. Hamsters were fed regular rodent chow ad libitum for a 2-week acclimatization period before being randomly assigned to one of 4 different diets, including (i) non-cholesterol control (NCC), (ii) $0.1 \%$ cholesterol control (CC), (iii) $0.1 \%$ cholesterol diet supplemented with $275 \mathrm{mg} / \mathrm{kg}$ diet of SCP1 and (iv) $0.1 \%$ cholesterol diet supplemented with $275 \mathrm{mg} / \mathrm{kg}$ diet of SCP2 (Degussa Bioactives, Germany). The composition of the animal feed is detailed in Table 3.1. The four diets were adapted from the American Institute of Nutrition (AIN) 93-G formula of a purified diet for experimental rodents and had similar nutrient compositions (Reeves, 1997). SCP pills were crushed and mixed thoroughly with the fat component of the diet prior to incorporation in the rest of the mixture. The alcohol composition of the SCP1 brand was 66-67\% octacosanol, and 33-34\% of other alcohols (Mas, 2000). The composition of the SCP2 brand was $68.8 \%$ octacosanol and 31.2\% of other alcohols (Degussa Bioactives product information).

\subsubsection{Protocol}

Hamsters were systematically randomized according to their body weights and assigned to the 4 experimental diets for a period of 28 days. Each of the four diet groups consisted of 12 hamsters. During randomization, animals were divided into 6 blocks of 8 hamsters on the 4 different treatments. This randomization procedure ensured a good distribution of body weights on the different treatments administered.

Body weights were recorded once a week while food intake was measured every three days. Average intakes were calculated by subtracting leftovers and spillage from the food portion given to the animals. On day 28 , hamsters were sacrificed by decapitation after carbon dioxide inhalation and trunk blood was collected in EDTA tubes and placed on ice. Blood was then centrifuged and plasma was separated and stored at $-20^{\circ} \mathrm{C}$.

\subsubsection{Plasma lipid analysis}

Blood samples from day 28 were used to determine lipid profiles. Plasma TC, high density lipoprotein cholesterol (HDL-C) and triglyceride (TG) concentrations were analysed with 
enzymatic kits, standardized reagents, and standards using a VP Autoanalyzer (Abbott Laboratories, North Chicago, IL, USA). Non-HDL-C (N-HDL-C) was calculated for hamsters using the following equation: $(\mathrm{N}-\mathrm{HDL}-\mathrm{C})=(\mathrm{TC})-(\mathrm{HDL}-\mathrm{C})$.

\subsubsection{Statistical analysis}

Sample size per group $(\mathrm{n}=12)$ was calculated to provide a power of $80 \%$ based on the smallest effect size on plasma TC seen in previous literature. The effect of diet groups on TC, HDL-C and TG was examined using a one-way ANOVA with a statistical significance set at $\mathrm{p}<0.05$. The block effect was included as part of the statistical model to rule out any effect of age on blood lipids. Additionally, effects of body weight and food intake on plasma lipids were tested as covariates to the effect of policosanol treatments. All data are presented as mean \pm SEM.

\subsection{Results}

No behavioural or physical abnormalities were recorded in hamsters throughout the trial period. None of the animals died nor escaped during the experiment.

\subsubsection{Growth of hamsters across the study period}

Weight patterns across the study period are illustrated in Figure 3.1. Average initial weights of hamsters in the NCC, CC, SCP1 and SCP2 groups were 109.6 $\pm 0.64,109.7 \pm 0.64$, $109.6 \pm 0.64$ and $109.4 \pm 0.88 \mathrm{~g}$ respectively. Hamster growth was higher in the first three weeks and decreased towards the end of the feeding period. Body weights in the NCC, CC, SCP1 and SCP2 groups increased by $17.41 \%, 17.6 \%, 19.2 \%$ and $15.7 \%$ respectively as compared to baseline. No significant differences were observed amongst groups for weight changes across the feeding period. Endpoint average weights in the NCC, CC, SCP1 and SCP2 groups were $126.4 \pm$ $1.82,129.0 \pm 1.82,130.3 \pm 2.05$, and $125.4 \pm 2.35 \mathrm{~g}$, respectively. When compared across groups, body weights recorded each feeding week did not differ significantly.

\subsubsection{Food intake of hamsters throughout the feeding period}

Average food intake of animals throughout the feeding period was $7.68 \pm 0.19 \mathrm{~g}, 7.82 \pm$ $0.18,7.59 \pm 0.21$ and $7.46 \pm 0.13 \mathrm{~g}$ in the $\mathrm{NCC}, \mathrm{CC}, \mathrm{SCP} 1$ and $\mathrm{SCP} 2$, respectively, as presented 
in Figure 3.2. Average food intakes did not differ significantly across the four diet groups. The daily amounts of policosanols ingested by the SCP1 and SCP2 treatment groups were 20.1 and $20.5 \mathrm{mg} / \mathrm{kg}$ body weight, respectively.

\subsubsection{Growth and intake of hamsters versus plasma lipids}

Body weight of hamsters recorded every week throughout the study period was not significantly correlated with plasma TC, HDL-C and TG values. Similarly, there was no association of daily feed intake with plasma lipids in hamsters.

\subsubsection{Lipid values as a result of policosanol feeding}

Plasma TC, HDL-C, N-HDL-C and TG endpoint values across treatments and percent differences from control groups are outlined in Table 3.2. There was no significant difference between the effect of SCP1 and SCP2 administration on plasma TC endpoints. Lower TC values were recorded in hamsters consuming the policosanol diets as compared to the CC group, however these differences were not statistically significant.

Similarly to TC, calculated N-HDL-C values were not significantly different in the SCP1 and SCP2 groups. Also, when compared to CC, the 5.3\% and 9.6\% reductions in N-HDL-C as a result of SCP1 and SCP2 treatments were not statistically significant. Endpoints in the policosanol groups were higher than in the NCC group, however non significant.

HDL-C and TG endpoints were not significantly affected by the SCP1 and SCP2 treatments as compared to the $\mathrm{CC}$ group. However, when compared to the NCC group, mean plasma TG in hamsters on the SCP1 diet was $68 \%$ higher than the mean in the NCC group.

\subsection{Discussion}

Results of the present study show, for the first time, that neither the Dalmer Cuban nor the alternative Degussa policosanol mixtures possess any significant effect on plasma TC, HDL$\mathrm{C}$ and TG levels over the moderate term in the hamster model. These results are in contrast with previous research conducted on the Dalmer mixture that demonstrates reductions in total cholesterol and LDL-cholesterol of more than 13\% (Varady et al., 2003). Studies conducted on rabbits, dogs and monkeys agree with the conclusion that policosanols reduce levels of LDL-C and TC significantly at dosages ranging from $5 \mathrm{mg}-500 \mathrm{mg} / \mathrm{kg}$ body weight/day (Arruzazabala $e t$ 
al., 1994; Mesa et al., 1994; Rodriguez-Echenique et al., 1994; Menendez et al., 1996;

Menendez et al., 1997). The hamster model used in the present study is considered to be one of the most suitable models for studying lipid lowering mechanisms (Kris-Etherton \& Dietschy, 1997) and has shown to resemble humans with regard to regulation of LDL-C metabolism (Spady \& Dietschy, 1988). One could argue that the absence of effect on N-HDL-C could be accounted for by the fact that hamsters carry a larger proportion of plasma cholesterol in HDL versus Non-HDL particles. Nonetheless, we would have expected to see changes in TC and NHDL-C similar to those seen in numerous studies examining the effect of LDL-C lowering substances in hamsters including phytosterols, statins and others (Otto et al., 1995; Field et al., 2004; Doggrell, 2005; Ebine et al., 2005).

Our results agree with the study conducted in 2003 by Wang et al (Wang et al., 2003), which compared the efficacy of rice and non-Dalmer SCP to that of plant sterols, both given in the context of a hypercholesterolemic diet. Hamsters in the policosanol groups did not show changes in TC and N-HDL-C as compared to cholesterol control, while the plant sterol group demonstrated TC levels significantly lower than control and policosanol groups by $22 \%$ and $30 \%$, respectively. Our results also agree with another study conducted in 2004 by Murphy et al. (Murphy et al., 2004), not using the hamster model, but providing rabbits with an alternative mixture of SCP, and showing no shifts in plasma lipids. In the current study, inclusion of the NCC group in the analysis ensured that our hamster model did respond to dietary intervention. In fact, this was manifested by a significant difference in plasma TC and N-HDL-C between the $\mathrm{NCC}$ and the CC group.

Studies on the effect of SCP supplementation on cholesterol metabolism have established that cholesterol-lowering effects, mainly mediated by a decrease in serum LDL-C levels, were correlated with the inhibition of hepatic cholesterol synthesis (Menendez et al., 1994; Menendez et al., 1997; Fernandez et al., 2001) and the stimulation of receptor-mediated LDL uptake by the liver (Menendez et al., 1996; Menendez et al., 1997). It could therefore be argued that the absence of effect shown in the study by Wang et al in 2003 was partly caused by the high cholesterol content of the diet $(0.25 \%$ by weight $)$ which could have maximally suppressed cholesterol biosynthesis. Although statins, which also impact cholesterol synthesis, were shown to be effective in the context of a high cholesterol diet (Kobayashi et al., 1989), this issue remains a point of concern in terms of the effect of dietary cholesterol on cholesterol 
biosynthesis (Jones et al., 1996). Consequently, the proportion of cholesterol used in the feed for the present study was $0.1 \%$ by weight, in order to reduce the suppression of cholesterol biosynthesis potentially masking the effect of policosanols. This choice of cholesterol content was also made to mimic a human cholesterol intake.

Cholesterol synthesis measurements were conducted using ${ }^{14} \mathrm{C}$ acetate in the in vitro studies mentioned above (Menendez et al., 1994; Gamez et al., 2001). The authors have attributed the reduction in ${ }^{14} \mathrm{C}$ concentration in fibroblasts treated with SCP to their inhibitory effect on the conversion of acetate to mevalonate leading eventually to a decrease in cholesterol synthesis (Menendez et al., 1994; Menendez et al., 2001a). In 2006, the same method was used by Singh et al in an in vitro study examining the effect of a policosanol formulation on cholesterol synthesis in human fibroblasts (Singh et al., 2006). The authors were able to observe a 30\% reduction in cholesterol labeling, agreeing with results obtained by the original research group. However, adding labeled acetate to a cell medium containing policosanols may lead to the dilution of labeled acetate as a result of policosanol metabolism, therefore falsely indicating a reduction of cholesterol synthesis at the level of acetate incorporation. It is thus possible that the methods used to date may have been inappropriate for the determination of policosanol metabolism and its impact on cholesterol synthesis. In fact, the newer independent human studies clearly showing that policosanols from Cuba or other sources has no true effect on the lipid profile of hypercholesterolemic humans (Lin et al., 2004; Reiner et al., 2005; Tedeschi-Reiner et al., 2005; Berthold et al., 2006; Greyling et al., 2006; Kassis \& Jones, 2006) support the hypothesis that the suppression observed in vitro is artificial.

The composition of alternative policosanol mixtures used by external research groups in most animal and human studies raised criticism in terms of the efficacy of any composition that diverges from the original Cuban mixture. Although the main active compound octacosanol is found in similar amounts in both Dalmer and alternative mixtures (between 60 and 70\%), products with different alcohol compositions appear to have different lipid-lowering activities despite their similar octacosanol contents (Castano et al., 2002b). In order to avoid the limitations pertaining to product authenticity, the present study used the original Cuban mixture manufactured by Dalmer laboratories in La Havana, Cuba, and compared it to an alternative policosanol mixture. Nevertheless, our data shows that hamsters responded to neither treatment, reinforcing the conclusions seen in studies showing no effect. In fact, our results agree with two 
recent human studies conducted in independent laboratories using the Dalmer product and reporting no change in TC and LDL-C in hypercholesterolemic individuals (Berthold et al., 2006; Kassis \& Jones, 2006).

The outcomes of the present trial should not be over-interpreted without considering the differences in methodology between this study and animal studies showing positive results. The effect of policosanols on lipid metabolism might differ amongst different animal species, therefore, the use of the hamster model in this trial might account for the lack of effect of policosanols, as compared to other studies using species such as rats and rabbits (Menendez et al., 1996; Menendez et al., 1997). However, noting that hamsters resemble humans in terms of LDL metabolism, data obtained using this model should be more applicable to humans than would be the case using some other models.

In most animal trials showing positive outcomes, hypercholesterolemia was induced endogenously through enhancement of cholesterol biosynthesis using a fat-free wheat starchcasein diet. In the present trial, hypercholesterolemia was induced exogenously through the addition of $0.1 \%$ cholesterol in the animals' diet. Since policosanols are believed to act at the level of cholesterol synthesis in the liver (Menendez et al., 1994; Menendez et al., 1997; Fernandez et al., 2001), the difference in the baseline biosynthesis induced by the diets could have accounted for the variation in results. Again, the diet used in the present study is more easily applicable to a human situation as compared to a diet free of fat and cholesterol.

\subsection{Conclusion}

In conclusion, the results of the present study show that both Dalmer Cuban SCP and alternative mixtures such as Degussa SCP, fail to reduce plasma lipid levels in hamsters. These outcomes question the efficacy of policosanols as lipid-lowering agents and emphasize the need to explore mechanisms underlying its effect on cholesterol biosynthesis and absorption.

\subsection{Acknowledgments}

We would like to thank Gordon Bingham for his technical help during the clinical trial. 
Table 3.1. Composition of the hamster diet

\begin{tabular}{lllll}
\hline \multicolumn{4}{l}{ Diet groups } \\
\hline Constituents (\% weight) & NCC & CC & SCP 1 & SCP 2 \\
\hline Casein & $20.0 \%$ & $20.0 \%$ & $20.0 \%$ & $20.0 \%$ \\
Corn starch & $26.0 \%$ & $26.0 \%$ & $25.8 \%$ & $26.1 \%$ \\
Sucrose & $33.2 \%$ & $33.2 \%$ & $33.0 \%$ & $33.1 \%$ \\
Beef tallow/Safflower oil mix & $10.0 \%$ & $10.0 \%$ & $10.0 \%$ & $10.0 \%$ \\
Cellulose & $5.0 \%$ & $5.0 \%$ & $5.0 \%$ & $5.0 \%$ \\
DL-methionine & $0.5 \%$ & $0.5 \%$ & $0.5 \%$ & $0.5 \%$ \\
Mineral mixture ${ }^{*}$ & $4.0 \%$ & $4.0 \%$ & $4.0 \%$ & $4.0 \%$ \\
Vitamin mixture ${ }^{\S}$ & $1.0 \%$ & $1.0 \%$ & $1.0 \%$ & $1.0 \%$ \\
Choline bitartrate & $0.2 \%$ & $0.2 \%$ & $0.2 \%$ & $0.2 \%$ \\
Butylated hydroxytoluene & $0.0 \%$ & $0.0 \%$ & $0.002 \%$ & $0.002 \%$ \\
Cholesterol & $0.0 \%$ & $0.1 \%$ & $0.1 \%$ & $0.1 \%$ \\
Dalmer policosanol powder $^{\mathrm{a}}{ }^{\mathrm{b}}$ & - & - & $0.5 \%$ & - \\
Degussa policosanol powder $^{\mathrm{b}}$ & - & - & & $0.03 \%$ \\
\hline NCC: Non-cholester & & & & \\
\hline
\end{tabular}

NCC: Non-cholesterol control; CC: Cholesterol control; SCP1: OriginalCuban sugar cane policosanols; SCP2: Alternative sugar cane policosanols. ${ }^{\text {a }}$ Purity of Dalmer policosanol product $=$ $6.0 \%$; ${ }^{b}$ Purity of Degussa policosanol product $=92.0 \%$.

*Mineral mixture composition (per kg): $500 \mathrm{~g}$ calcium phosphate dibasic, $74 \mathrm{~g}$ sodium chloride, $220 \mathrm{~g}$ potassium citrate monohydrate, $52 \mathrm{~g}$ potassium phosphate, $24 \mathrm{~g}$ magnesium oxide, $3.5 \mathrm{~g}$ manganese carbonate, $6 \mathrm{~g}$ ferric citrate, 1.6 zinc carbonate, $0.3 \mathrm{~g}$ cupric carbonate, $0.01 \mathrm{~g}$ potassium iodate, $118 \mathrm{~g}$ sucrose.

${ }^{\S}$ Vitamin mix composition (per kg): 600mg thiamine hydrochloride, $600 \mathrm{mg}$ riboflavin, $700 \mathrm{mg}$ pyridoxine hydrochloride, $3 \mathrm{~g}$ nicotinic acid, $1.6 \mathrm{~g}$ D-calcium pantothenate, $200 \mathrm{~g}$ folic acid, $20 \mathrm{mg}$ D-biotin, $1 \mathrm{mg}$ cyanocobalamin, $1.6 \mathrm{~g}$ retinyl palmitate, DL- $\alpha$-tocopherol acetate, $250 \mathrm{mg}$ cholecalciferol, $5 \mathrm{mg}$ melaquinone, $973 \mathrm{~g}$ sucrose. 
Table 3.2. Plasma lipids (Mean \pm SEM) in response to policosanol diets as compared to controls

\section{Concentration (mmol/l) $\quad$ Change from NCC (\%) Change from CC (\%)}

\begin{tabular}{|c|c|c|c|}
\hline \multicolumn{4}{|l|}{ TC } \\
\hline $\mathrm{NCC}$ & $4.78^{a} \pm 0.21$ & - & -24.4 \\
\hline $\mathrm{CC}$ & $6.33^{b} \pm 0.21$ & 32.4 & - \\
\hline SCP1 & $5.80^{b} \pm 0.21$ & 21.3 & -8.37 \\
\hline SCP2 & $5.67^{a b} \pm 0.28$ & 18.6 & -10.4 \\
\hline \multicolumn{4}{|c|}{ HDL-C } \\
\hline $\mathrm{NCC}$ & $2.60^{a} \pm 0.17$ & - & -15.9 \\
\hline $\mathrm{CC}$ & $3.09^{a} \pm 0.17$ & 18.8 & - \\
\hline SCP1 & $2.73^{a} \pm 0.17$ & 5.00 & -11.6 \\
\hline $\mathrm{SCP} 2$ & $2.74^{a} \pm 0.23$ & 5.38 & -11.3 \\
\hline \multicolumn{4}{|c|}{ Non-HDL-C } \\
\hline $\mathrm{NCC}$ & $2.18^{c} \pm 0.28$ & - & -32.7 \\
\hline $\mathrm{CC}$ & $3.24^{d} \pm 0.28$ & 48.6 & - \\
\hline SCP1 & $3.07^{c d} \pm 0.28$ & 40.8 & -5.3 \\
\hline SCP2 & $2.93^{c d} \pm 0.36$ & 34.4 & -9.6 \\
\hline \multicolumn{4}{|l|}{ TG } \\
\hline $\mathrm{NCC}$ & $2.38^{a} \pm 0.28$ & - & -25.4 \\
\hline $\mathrm{CC}$ & $3.19^{a b} \pm 0.28$ & 34.0 & - \\
\hline SCP1 & $4.00^{b} \pm 0.36$ & 68.1 & 25.4 \\
\hline SCP2 & $3.41^{a b} \pm 0.28$ & 43.3 & 6.9 \\
\hline
\end{tabular}

NCC: Non-cholesterol control; CC: Cholesterol control; SCP1: originalCuban sugar cane policosanols; SCP2: Alternative sugar cane policosanols; $\mathrm{n}=44$.

${ }_{a, b}$ Means within a lipid category with different superscript letters are statistically significant at $\mathrm{p}<0.01$

${ }^{c, d}$ Means within a lipid category with different superscript letters are statistically significant at $p<0.05$. 
Figure 3.1. Body weights (Mean \pm SEM) across the feeding period

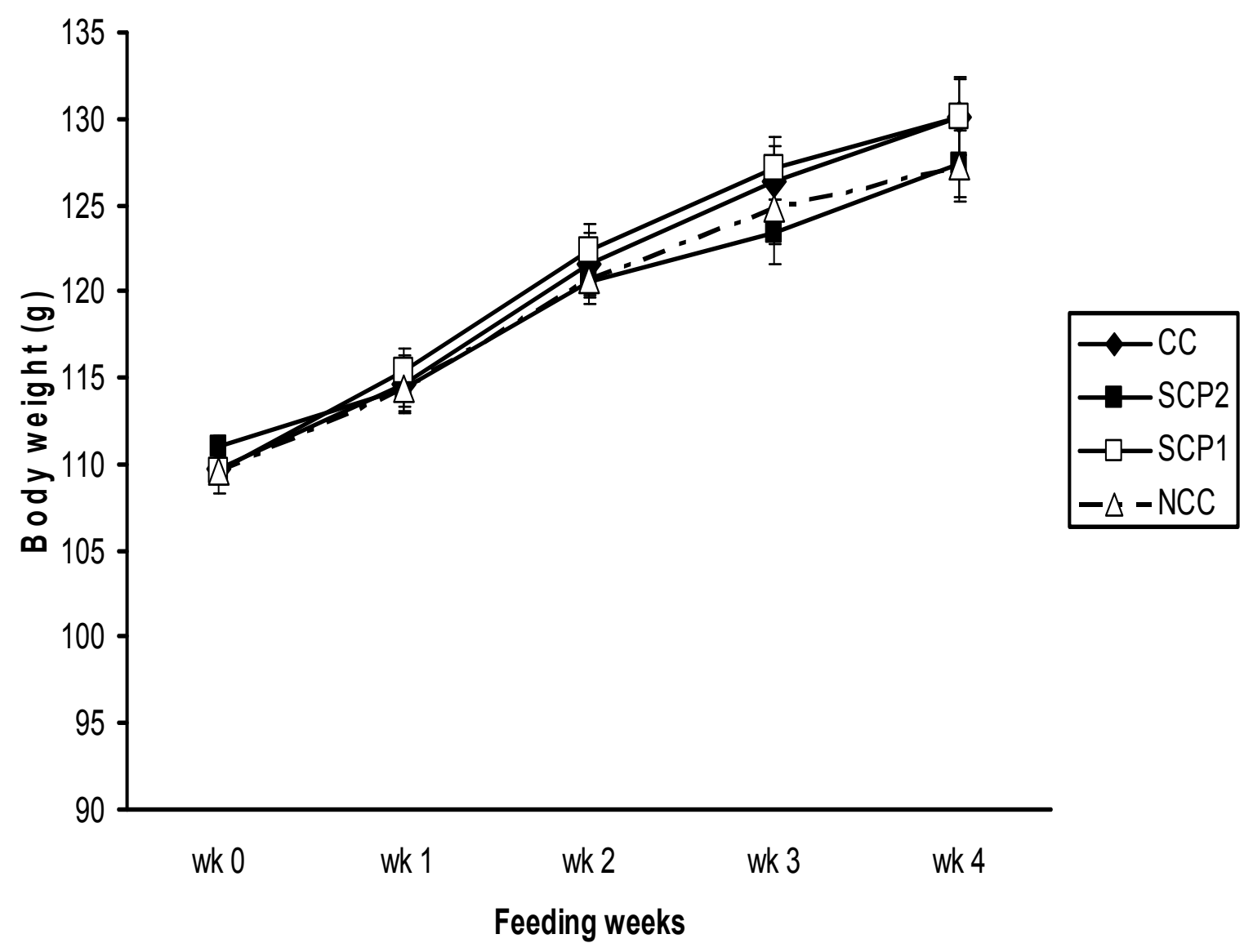

Fig. 3.1: NCC: Non-cholesterol control; CC: Cholesterol control; SCP1: Dalmer Cuban sugar cane policosanols; SCP2: Alternative sugar cane policosanols; $\mathrm{n}=44$. No significant difference in weight amongst the four groups at the different time points 
Figure 3.2. Average intake of hamsters in different diet groups throughout the study period

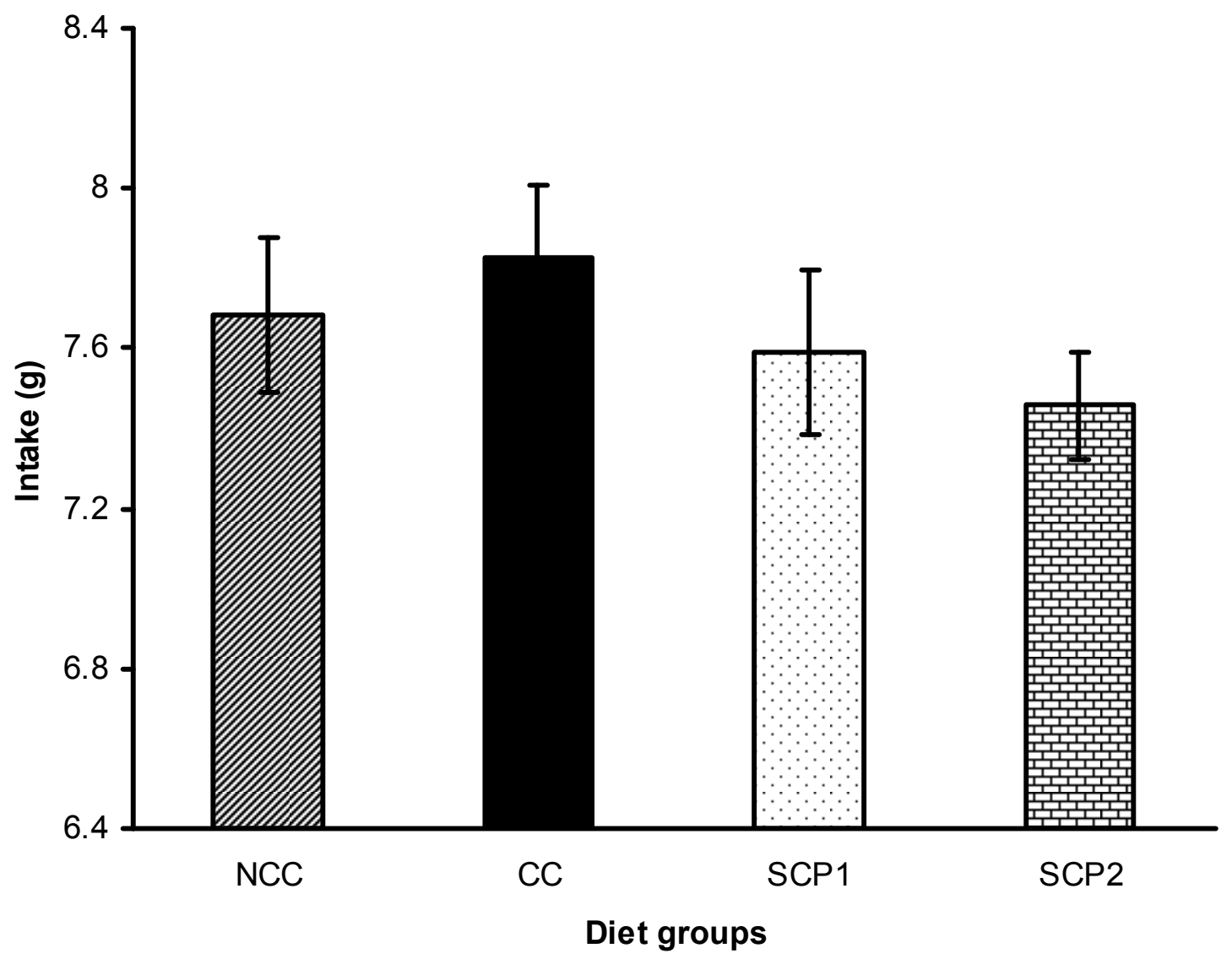

Figure 3.1: NCC: Non-cholesterol control; CC: Cholesterol control; SCP1: Dalmer Cuban sugar cane policosanols; SCP2: Alternative sugar cane policosanols; $n=44$. No significant difference in average intake amongst the four groups 


\section{Bridge 1}

Manuscript 1 demonstrated no difference in the cholesterol-lowering effect of two different brands of sugar cane policosanols. More importantly, it showed that the original Cuban policosanol product, previously reported to result in substantial reductions in over 60 studies (Varady et al., 2003), induced no significant decreases in plasma cholesterol, in golden Syrian hamsters. Policosanols can be extracted from numerous sources including rice, beeswax, wheat germ and sugar cane, the latter (SCP) being the only source showing significant cholesterollowering potential. In addition, commercial natural health product preparations are characterized by a large variability in composition across different brands even when originating from the same source. Although octacosanol is thought to be the main active component of SCP mixtures (Mas, 2000), minor alcohol composition seems to affect cholesterol-lowering efficacy as well (Castano et al., 2002b; Singh et al., 2006). Since factors such as extraction procedure may affect the alcohol composition of SCP mixtures (Wang et al., 2007), it is crucial to determine the variability in alcohol distribution of preparations produced by different manufacturers as well as differences within products of the same brand. The objective of the animal trial component of the present project was to compare the efficacy of two different brands of sugar cane policosanols under the same experimental conditions in order to determine the role of the alcoholic composition of the policosanol extract. In Manuscript 1, no significant effect was observed on cholesterol levels and synthesis or absorption in golden Syrian hamsters, in the cases of both original and alternative SCP mixtures. Since the inhibitory action of SCP on cholesterol biosynthesis is thought to be directed towards the hepatic peroxisomes (Jakobs \& Wanders, 1991), the metabolism of SCP in general and its absorption efficiency in particular are of great interest for a better evaluation in terms of its cholesterol-lowering efficacy. Accordingly, the objective of the following manuscript was to present a compositional analysis of different SCP

mixtures as well as an assessment of the metabolism of two different brands of SCP administered to golden Syrian hamsters. 


\title{
Chapter 4
}

\section{Manuscript 2: Comparison of composition and absorption of sugar cane policosanols}

Christopher P.F. Marinangeli ${ }^{a}$, Amira N. Kassis ${ }^{a}$, Deepak Jain ${ }^{a}$, Naoyuki Ebine ${ }^{a}$, Stephen C. Cunnane $^{\mathrm{b}}$, and Peter J.H. Jones ${ }^{\mathrm{a}}$

\author{
Author affiliation: \\ ${ }^{\mathrm{a}}$ School of Dietetics and Human Nutrition, McGill University, Ste. Anne de Bellevue, Québec, \\ Canada \\ ${ }^{\mathrm{b}}$ Research Centre on Aging, Université de Sherbrooke, Sherbrooke, Québec, Canada
}

This manuscript is has been published in the British Journal of Nutrition

Marinangeli CP, Kassis AN, Jain D, Ebine N, Cunnane SC, Jones PJ. Comparison of composition and absorption of sugarcane policosanols. Br J Nutr. 2007 Feb;97(2):381-8

This project was funded by the Advanced Foods and Materials Network- Network of Centers of Excellence 
Chapter 4 : Manuscript 2

\section{Manuscript outline}

4.1. Abstract

4.2. Introduction

4.3. Materials and methods

4.3.1. Compositional analysis

4.3.1.1. Original and alternative sugar cane PC products

4.3.1.2. PC tablet and powder analysis

4.3.2. Animal trial

4.3.2.1. Animals and diets

4.3.2.2. Tissue and plasma analysis

4.3.2.3. Fecal analysis

4.3.2.4. Gas chromatography analysis

4.3.3. Statistical analysis

4.4. Results

4.4.1. Compositional analysis

4.4.2. Animal trial

Animal tissue, plasma, and fecal analysis

4.5. Discussion

4.6. Conclusion

4.7. Acknowledgments 


\subsection{Abstract}

Background: Policosanols (PC) exist as very long chain alcohols derived from sugarcane currently used in many countries as a cholesterol-lowering therapy. PC purity and relative percent composition have been suggested as primary reasons that the original Cuban PC (OPC) supplements possess lipid lowering efficacy.

Objectives: The purpose of this study was to first compare the relative percent purity and PC composition of both OPC and alternative sources of PC (APC). A second objective was to feed Syrian hamsters a diet containing $0.275 \mathrm{mg}$ PC/g of either the OPC or an APC product (APC1) and compare subsequent tissue, plasma and fecal PC levels.

Methods: Five animals from the APC1 dietary group received diet containing 10 times the original amount of PC.

Results: Results indicate that the APC formulations have a composition that is highly consistent with the OPC supplement, with octacosanol being present within the cited $60-70 \%$ range. PCs were undetectable in the small intestine, liver, adipose, or plasma in animals fed either source. Hamsters fed OPC excreted octacosanol (C28) more rapidly $(p<0.05)$ than hamsters receiving APC1.

Conclusion: If the cholesterol-lowering efficacy of PC mixtures is dependent on their purity and composition, then sugarcane-derived APC products should possess similar therapeutic properties as the OPC supplement. 


\subsection{Introduction}

Policosanols (PC) are mixtures of primary aliphatic 24 to 34 carbon alcohols derived from the wax constituent of plants. Researchers in Cuba isolated PC from sugarcane (Saccharum officinarum, L.) wax and developed the original PC supplement (OPC) (GouniBerthold \& Berthold, 2002); (Monograph, 2004), which was approved in 1991 in Cuba as a lipid lowering therapy (Gouni-Berthold \& Berthold, 2002). Since then, the OPC supplement has been the subject of numerous human clinical investigations. Daily doses ranging from 2 to $20 \mathrm{mg}$ have been shown to be efficacious in safely producing dose-dependant reductions in total cholesterol (TC) and low-density lipoprotein cholesterol (LDL-C) ranging from 10 to $23 \%$ and 11 to 31\%, respectively (Mas, 2000; Gouni-Berthold \& Berthold, 2002). Studies also show that $\mathrm{PC}$ are at least equally effective in lowering both TC and LDL-C as are prescription medications (Ortensi et al., 1997; Pons et al., 1997; Alcocer et al., 1999; Crespo et al., 1999; Castano et al., 2000a; Castano et al., 2003b). Animal and cell culture studies have demonstrated that PC reduce cholesterol synthesis either by interfering with the production, degradation or activity of HMGCoA reductase (Menendez et al., 1996; Menendez et al., 2001a; Singh et al., 2006), as well as by increasing cellular LDL uptake (Menendez et al., 1997).

Despite the impressive initial results for PC, for the most part, research groups outside of Cuba using alternative sources of PC (APC) derived from either sugarcane or non-sugarcane sources have been unable to reproduce the initial reports in animals (Wang et al., 2003; Murphy et al., 2004; Wang et al., 2005) or in humans (Lin et al., 2004; Reiner et al., 2005; Berthold et al., 2006; Greyling et al., 2006). The relative composition of the OPC supplement has been published indicating a purity of $\geq 90 \%$ with octacosanol (C-28) being the most abundant PC (6070\%), followed by triacontanol (C-30;10-15\%), hexacosanol (C-26; 4.5-10\%), dotriacontanol (C-32; 3-8\%), heptacosanol (C-27; $\leq 5 \%)$, tetracosanol (C-24; $\leq 2 \%)$, nonacosanol (C-29; $\leq 2 \%)$, and tetratriacontanol (C-34; $\leq 2 \%)$ (Mas, 2000). It is thought that since octacosanol is present in the highest quantity, it is the primary PC responsible for lowering cholesterol levels (Mas, 2000). The original researchers have defended their results by indicating that it is the exclusive purity and relative composition of the OPC supplement that are responsible for its ability to reduce blood lipids (Castano et al., 2002b). A systematic comparison of OPC versus APC products has not, however, been undertaken. 
PC metabolism has also been the subject of controversy. Due to the structure of PC, their hydrophobicity appears to result in poor absorption (Hargrove et al., 2004), confirmed in both rats (Kabir \& Kimura, 1995; Menendez et al., 2005) and monkeys (Menendez et al., 2005) using radiolabelled $\left[8-{ }^{14} \mathrm{C}\right]$-octacosanol (Kabir \& Kimura, 1995)as well as in an octacosanol metabolism study using PC supplements (Menendez et al., 2005).

The purpose of the present study, therefore, was to first analyze various brands of the OPC and sugarcane-derived APC supplements and compare our analyses to the published data for the OPC product. A second objective was to evaluate and compare the plasma, tissue, and fecal levels of octacosanol within animals fed either OPC or an APC product. 


\subsection{Materials and methods}

\subsubsection{Compositional analysis}

\subsubsection{Original and alternative sugar cane PC products}

OPC tablets indicated as containing either 6 or $20 \mathrm{mg}$ of PC produced by Dalmer Laboratories were obtained from pharmacies in Cuba (OPC1 and OPC2) and Argentina (OPC3). OPC supplements were also obtained in Canadian natural health stores (OPC4 and OPC5). Sugarcane-derived APC products were provided as powders from Degussa Bioactives (Champaign, IL) (APC1), Garuda International Inc. (Lemon Cove, CA) (Lesstanol®) (APC2), and AHD International, LLC (Atlanta, GA) (APC3).

\subsubsection{PC tablet and powder analysis}

Individual tablets were weighed, crushed and placed in a test tube with chloroform. The powder-chloroform mixture was heated for $15 \mathrm{~min}$ at $60^{\circ} \mathrm{C}$ and filtered into another test tube. The solution was dried under nitrogen gas and either $6 \mathrm{ml}$ (for $6 \mathrm{mg}$ tablets) or $10 \mathrm{ml}$ (for $20 \mathrm{mg}$ tablets) of chloroform was added to the dried sample and heated for $15 \mathrm{~min}$ at $60^{\circ} \mathrm{C}$. Aliquots of PC were transferred to another test tube. The solution was dried under nitrogen and $200 \mu 1$ of methylene chloride was added and heated for $15 \mathrm{~min}$ at $60^{\circ} \mathrm{C}$. The APC mixtures were prepared using the same procedure as above except a $20 \mathrm{mg}$ sample was used. Separation of PC was performed by gas chromatography (GC) using an Agilent 6890 GC (Mississauga, Canada) equipped with a flame ionization detector (FID) and a fused capillary column HP-1 (30m x $0.25 \mathrm{~mm}$ I.D., $0.25 \mu \mathrm{m}$ film thickness). The injector temperature was $330^{\circ} \mathrm{C}$ with a $50: 1 \mathrm{split}$. The initial oven temperature was $150^{\circ} \mathrm{C}$ for $2 \mathrm{~min}$, then increased at $20^{\circ} \mathrm{C} / \mathrm{min}$ to $250^{\circ} \mathrm{C}$, then to $325^{\circ} \mathrm{C}$ at $6^{\circ} \mathrm{C} / \mathrm{min}$. The carrier gas was helium at $1.0 \mathrm{ml} / \mathrm{min}$. Peaks were identified using standards for tetracosanol, hexacosanol, heptacosanol, octacosanol, and triacontanol obtained from Sigma-Aldrich (Oakville, Canada). Nonacosanol and dotriacontanol were identified by extrapolation, while tetratriacontanol was identified using beeswax purchased from SigmaAldrich (Oakville, Canada), plus information provided by Jimenez et al. (2003), and a certificate of analysis provided by Garuda International Inc. PC compositions were compared to those reported by Mas (2000) outlining the purity and composition of the OPC supplement manufactured by Dalmer Laboratories (Havana, Cuba). 


\subsubsection{Animal trial}

\subsubsection{Animals and diets}

This experiment was approved by the McGill Animal Care and Research Ethics committee in accordance with guidelines of the Canadian Council on Animal Care. The Syrian hamster was selected because it has been considered one of the best animal models for examining cholesterol metabolism (Kris-Etherton \& Dietschy, 1997). Thirty-six Golden Syrian hamsters weighing 80 to $100 \mathrm{~g}$ were housed in individual cages. Animals were acclimatized to a 12-h light/dark cycle and fed regular rodent chow for a period of 2 weeks. All animals had free access to water for the entire duration of the investigation. Hamsters were randomly assigned to 1 of 3 experimental diets prepared according to the AIN-93G formula. Study diet 1 was a control diet consisting of casein $(20 \%)$, cornstarch $(26 \%)$, sucrose $(33 \%)$, beef tallow/safflower oil mix (10\%), cellulose (5\%), DL-methionine (0.5\%), mineral mixture $(4 \%)$, vitamin mixture $(1 \%)$, choline bitartrate $(0.2 \%)$, butylated hydroxytoluene $(0.002 \%)$, and cholesterol $(0.1 \%)$. Dietary groups 2 and 3 received the same dietary formulation but with the addition of sugarcane PC at $0.275 \mathrm{mg} / \mathrm{g}$ of diet. Dietary group 2 (APC1) was given sugarcane PC provided by Degussa Bioactives (Champaign, IL), while dietary group 3 (OPC) was given sugarcane PC manufactured by Dalmer Laboratories (Havana, Cuba). OPC was obtained in tablet form, crushed into a fine powder, and subsequently added to the diet. Five animals from the APC1 group were given diet containing 10 times the original amount $(2.75 \mathrm{mg}$ PC/g diet) in order to observe whether elevated PC intake results in a proportional increase in plasma and tissue levels of PC. All animals were given free access to the experimental diets for the duration of the study. Food intake and food waste were recorded every 3 days. Body weights were recorded every 7 days. After 28 days on their experimental diets, animals were anesthetized by carbon dioxide inhalation. Blood was collected via decapitation and plasma was separated from red blood cells. The perirenal adipose tissue, liver, and small intestine were immediately collected. Plasma and all tissues were stored at $-80^{\circ} \mathrm{C}$. Feces were collected for each animal over the final 3 days, immediately dried and stored at $-20^{\circ} \mathrm{C}$. On these 3 days, leftover food and food waste was recorded daily to determine the daily intake. 


\subsubsection{Tissue and plasma analysis}

Samples of liver, adipose, and small intestine were homogenized in saline and extracted using chloroform: methanol (2:1) (Folch et al., 1957). Plasma was analyzed using the same method. The samples were heated and shaken for $10 \mathrm{~min}$ at $60^{\circ} \mathrm{C}$ and then centrifuged at 1500 $\mathrm{rpm}$ at $25^{\circ} \mathrm{C}$ for $5 \mathrm{~min}$ to maintain solubility of the PC. This extraction procedure was repeated 3 times. Extracts were dried under nitrogen and saponified using methanolic $\mathrm{KOH}$ at $90^{\circ} \mathrm{C}$ for $2 \mathrm{~h}$ with concurrent shaking. Total lipids were then extracted with 3 washes of petroleum ether. After each addition of petroleum ether, the saponified solution was simultaneously shaken and heated at $60^{\circ} \mathrm{C}$ and then centrifuged for $5 \mathrm{~min}$ at $25^{\circ} \mathrm{C}$. Extracts were then dried under nitrogen. Methylene chloride was added to the tissue extracts for GC analysis. $5 \alpha$-cholestane (SigmaAldrich, Oakville, Canada) was added as an internal standard for the quantification of PC.

\subsubsection{Fecal analysis}

Samples of feces from each of the three days were ground into a fine powder. Fecal PC were extracted and quantified by the same method as outlined above. Data were interpreted by dividing the 3-day average amount of octacosanol excreted by the 3-day average amount of octacosanol consumed.

\subsubsection{Gas chromatography analysis}

Before samples were analyzed the detection limits for PC of our GC were determined. Standards of tetracosanol, hexacosanol, heptacosanol, octacosanol and triacontanol were purchased from Sigma-Aldrich. Each standard was mixed with chloroform, making individual stock solutions of $1 \mathrm{mg} / \mathrm{ml}$ with $1 \mathrm{ml}$ aliquots of each solution being isolated. Serial dilutions using chloroform as the solvent produced final concentrations that were $1 / 4,1 / 16,1 / 64,1 / 256$, $1 / 1024,1 / 1536,1 / 4096$ of the original stock solution. Aliquots of $1 \mathrm{ml}$ of each dilution were isolated, and $50 \mu \mathrm{l}$ of a $1 \mathrm{mg} / \mathrm{ml}$ stock solution of $5 \alpha$-cholestane was added to each test tube as an internal standard. Samples were dried under nitrogen and re-dissolved in methylene chloride with sonication. Preceding the analysis of sets of serial dilutions, a mixed standard containing $5 \alpha$-cholestane $(0.12 \mathrm{mg} / \mathrm{ml})$, tetracosanol $(0.12 \mathrm{mg} / \mathrm{ml})$, hexacosanol $(0.12 \mathrm{mg} / \mathrm{ml})$, octacosanol $(0.12 \mathrm{mg} / \mathrm{ml})$, and triacontanol $(0.12 \mathrm{mg} / \mathrm{ml})$ was analyzed. Results from these chromatograms were used for quantifying PC found in samples evaluated that same day. 
Separation was achieved using a Hewlett Packard 5890 GC equipped with an FID and fused capillary column SAC-5 (30m x 0.25mm I.D., $0.25 \mu \mathrm{m}$ film thickness) from Sigma-Aldrich (Oakville, Canada). The injector temperature was $300^{\circ} \mathrm{C}$ and initial oven temperature was $160^{\circ} \mathrm{C}$. The oven temperature was increased to $245^{\circ} \mathrm{C}$ at $15^{\circ} \mathrm{C} / \mathrm{min}$ and held for $4 \mathrm{~min}$. The temperature was again increased to $280^{\circ} \mathrm{C}$ at $15^{\circ} \mathrm{C} / \mathrm{min}$. After $4 \mathrm{~min}$, a final temperature of $305^{\circ} \mathrm{C}$ was reached at a rate of $40^{\circ} \mathrm{C} / \mathrm{min}$ and held for $11 \mathrm{~min}$. Helium was used as the carrier gas. Samples were quantified using the internal standard method (Scott \& Perry, 1998). Since serial dilutions were used, the calculated concentrations for each dilution were log-transformed when presented.

Tissue, plasma, and fecal samples were analyzed using the same GC protocol. On each day of analysis the same mixed standard as mentioned above was analyzed for the quantification of octacosanol.

\subsubsection{Statistical analysis}

All statistical analyses were completed using SAS version 8 (SAS Institute Inc. Cary, NC). The PC content in all tissues and plasma were compared across the 3 treatment groups using one way analysis of variance (ANOVA). Significant differences between groups were assessed using the Tukey a priori test. For the fecal analysis, the average octacosanol intake as well as the average octacosanol excretion was determined over the three days in which feces was collected. The ratio between the average amount $(\mathrm{mg})$ of octacosanol excreted versus the average amount (mg) of octacosanol ingested was computed for each animal. Data were then subjected to the arcsine transformation to achieve normality. The resultant data were then analyzed by ANOVA and significant differences between groups were determined using the Tukey test. For reporting purposes, the arcsine transformed ratio estimates for each PC group was changed back to their original value. However, the reported $p$-value was taken from the transformed analysis. For all statistical analysis, data is presented as the mean $\pm \mathrm{SEM}$ and significance was established at $p<0.05$. 


\subsection{Results}

\subsubsection{Compositional analysis}

The results comparing various OPC supplements to the cited OPC purity and composition are summarized in Table 4.1. All of the OPC supplements met the specified requirements for the major PC constituents and the detectable minor constituents. One exception was with OPC2, which had a percent composition of hexacosanol that was slightly lower than the cited published range of 4.5-10\% (Mas 2000). None of the OPC supplements contained detectable levels of tetracosanol and tetratriacontanol. When APC was compared to the published composition of the original mixture, APC2 and APC3 had purities greater than the 90\%, while the APC1 formulation had a slightly lower value at 88.7\% (Table 4.1). Octacosanol, the major component thought to be responsible for cholesterol-lowering efficacy, was present within the 60 to $70 \%$ range for both APC2 and APC3, while the amount found in APC1 was only $1.6 \%$ below the cited $60 \%$ limit. Additionally, a notable departure from the original published range for triacontanol was observed in APC3.

\subsubsection{Animal trial}

Animal tissue, plasma, and fecal analysis

The GC detection limits for pure tetracosanol, hexacosanol, heptacosanol, octacosanol, and triacontanol were identified as $651.6 \mathrm{ng} / \mathrm{ml}, 431.8 \mathrm{ng} / \mathrm{ml}, 377.8 \mathrm{ng} / \mathrm{ml}, 402.1 \mathrm{ng} / \mathrm{ml}$, and $430.2 \mathrm{ng} / \mathrm{ml}$, respectively.

No differences in body weight or food intake were observed among animals for the duration of the study. PC was not detectable in tissues or plasma in any of the animals receiving either OPC or APC1 mixtures, even at an intake of $2.75 \mathrm{mg} / \mathrm{g}$ diet. Large amounts of ingested octacosanol were recovered within the feces. Compared to the amount of PC ingested, the total amount of octacosanol recovered for animals consuming either OPC or APC1 ranged from 24.7 to $89.7 \%$ (Figure 4.1). For animals consuming the diet containing 10 fold the amount of APC1 of the other groups, 43.9 to $51.7 \%$ of ingested octacosanol was recovered. Percent excretion of octacosanol was $43 \%$ higher $(p=0.01)$ in the animals consuming the OPC versus APC1 $(38.8 \%$ \pm 0.45 versus $55.8 \% \pm 0.29$ ) (Figure 4.1). The excretion of octacosanol was $37 \%$ lower in the animals receiving higher levels of APC1 compared to animals receiving OPC, coupled with a strong trend towards significance $(p=0.05)$. There was no difference $(p=0.83)$ in the amount of 
octacosanol excreted relative to the amount ingested between animals receiving the normal and higher levels of APC1.

\subsection{Discussion}

All sugarcane-derived PC analyzed in the present study demonstrated a similar PC purity and composition across brands. For the OPC supplements, a similar composition between brands indicates consistent manufacturing practices regardless of where the product is marketed. Nonetheless, contrary to the cited analysis of the OPC product, neither tetracosanol nor tetratriacontanol were detectable in any of the OPCs (Table 4.1). The cited compositional analyses of the OPC supplement classify tetracosanol and tetratriacontanol as minor constituents comprising of $<2 \%$ of the total PC mixture (Mas, 2000). Consequently, their importance in contributing to the cholesterol-lowering efficacy of the OPC supplement is unknown. Analysis of the APC1 showed an octacosanol composition and PC purity that was $<2 \%$ below the cited limits of the OPC product (Table 4.1). Whether this small reduction in octacosanol would substantially affect cholesterol-lowering efficacy seems unlikely.

One of the more important observations of this study concerns the composition of APC2. The APC2 formulation is Lesstanol ${ }^{\circledR}$, the same sugarcane-derived PC mixture previously compared to the OPC supplement by the original Cuban research group. Compared to OPC, Lesstanol ${ }^{\circledR}$ failed to reduce TC and LDL-C levels to the same extent as OPC (Castano et al., 2002b). The original researchers reported that Lesstanol ${ }^{\circledR}$ was less effective in reducing TC and LDL-C since compared to the OPC formulation, this APC mixture was shown to have a lower purity as well as a different PC composition, including notably lower levels of octacosanol (Castano et al., 2002a). Results from our study indicate that the purity and relative percent composition of the PC contained in APC2 are actually comparable to the OPC product. If PC efficacy is dependant on the relative percent composition of the PC formulation, then Lesstanol® would be expected to be as effective as the OPC supplement in lowering blood TC and LDL-C levels.

It has been suggested that PC could possibly affect the membrane fluidity of organelles responsible for manufacturing endogenous cholesterol, resulting in reduced synthesis or increased degradation of cellular HMG-CoA reductase (Menendez et al., 2001a). This theory stems from studies which outline that changes in the cellular and/or organelle membrane fatty 
acid composition, can manipulate membrane fluidity and specifically affect HMG-CoA reductase activity (Davis \& Poznansky, 1987; Whitcomb et al., 1988; Garcia-Pelayo et al., 2003) Perhaps the relative composition of PC mixtures can produce specific membrane fluidity changes in organelles responsible for endogenous cholesterol synthesis; more specifically, that the published compositional range concerning the OPC formulation may produce an exclusive membrane fluidity that would have the greatest effect on reducing HMG-CoA reductase activity. Another theory attempting to explain the action of PC on HMG-CoA reductase suggests that tricontanol activates AMP-kinase. An in vitro study by Singh et al. (2006) found that the addition of PC to cultured hepatoma cells significantly inhibited cholesterol synthesis. Thus far, octacosanol was thought to be the active component of the OPC supplement responsible for its cholesterol-lowering properties. When the individual PCs were tested, only tricontanol had an inhibitory effect. An increase in AMP-kinase phosphorylation was also noted (Singh et al., 2006). The researchers suggest that since phosphorylated AMP-kinase is a known inhibitor of HMG-CoA reductase, the activation of AMP-kinase by tricontanol found in PC supplements could be the mechanism behind PC cholesterol-lowering efficacy (Singh et al., 2006). Nonetheless, human PC supplementation studies conducted by external research groups using either OPC or APC products have failed to show cholesterol-lowering efficacy. In a recent human clinical trial, $20 \mathrm{mg} /$ day of the APC product Lesstanol ${ }^{\circledR}$ was administered to hypercholesterolemic patients. After 12 weeks of treatment, Lesstanol ${ }^{\circledR}$ failed to produce any changes in serum lipid levels (Greyling et al., 2006). However, a recent study by Berthold et al. (2006), was the first external research group to use OPC in hypercholesterolemic and hyperlipidemic patients. OPC was administered at 10,20,40, and $80 \mathrm{mg}$ /day to 4 different treatment groups over a 12 week period. Similar to the results reported by Greyling et al. (2006), OPC was unable to elicit significant reductions in TC and LDL-C compared to placebo. Nonetheless, the OPC used in the Berthold et al. (2006) study was purchased as a raw material, and then subsequently manufactured into tablets. To our knowledge, external research centers have yet to publish data testing PC efficacy in a clinical setting using the OPC product administered as supplemental tablets produced in Cuba.

Initially, dietary/supplemental PC is thought to be transported to the liver via chylomicrons (Hargrove et al., 2004). Next, they enter the endoplasmic reticulum and are metabolized to their corresponding acid via the fatty alcohol cycle using the enzyme alcohol: 
$\mathrm{NAD}^{+}$oxidoreductase (Lee, 1979; Rizzo et al., 1987). Research indicates that the mitochondria are unable to metabolize very long chain fatty acids (Jakobs \& Wanders, 1991; Mannaerts et al., 2000; Olivier \& Krisans, 2000; Wanders et al., 2000; Wanders et al., 2001). It has been shown that these molecules are partially metabolized or "chain shortened" by substrate specific $\beta$ oxidative enzymes contained in the peroxisome. The resulting acids can then be transported to the mitochondria for further processing (Jakobs \& Wanders, 1991; Mannaerts et al., 2000); (Olivier \& Krisans, 2000; Wanders et al., 2000; Wanders et al., 2001). This sequence of events is ideal for the proposed mechanism of action outlined above. It has been suggested that the PC corresponding acids are responsible for the observed therapeutic effect on lipid levels (Mendoza, 2001; Menendez et al., 2001b; Gamez, 2003). Thus, it is possible that some of the very long chain fatty acids produced from PC supplements are directly incorporated into the peroxisome membrane and perhaps affecting HMG-CoA reductase activity. The notion that PC lowers cholesterol through an effect on peroxisomes would be consistent with the fact that the peroxisome is the major site for endogenous cholesterol biosynthesis and contains the highest levels of HMG-CoA reductase (Hodge et al., 1991; Mandel et al., 1995; Olivier \& Krisans, 2000). However, for this to occur, adequate amounts of supplemental PC must be absorbed. In the present animal trial, our data indicate that supplemental PCs were undetectable in animals receiving either OPC or the APC1 formulation as well as animals receiving the diet containing 10 times the original amount of APC1. Furthermore, no changes in serum lipid levels were noted. The original researchers have demonstrated cholesterol-lowering efficacy of PC using a genetically diverse range of models including humans (Aneiros et al., 1995), monkeys (Rodriguez-Echenique et al., 1994), rabbits (Arruzazabala et al., 1994; Menendez et al., 1997), dogs (Mesa et al., 1994), and rats (Menendez et al., 1996). Hence, it is unlikely that our observations are due to the hamster model utilized in the present study. The hypothesis outlined above regarding PC composition and its mechanism for lowering HMG-CoA reductase activity in the liver would require that adequate amounts of the supplement is absorbed and hence would be detected in both the plasma and hepatocytes. Studies indicate that as both the chain length (Sallee \& Dietschy, 1973; Sallee, 1979; Bernard \& Carlier, 1991) and degree of saturation (Jones et al., 1985b) increase, the intestinal absorption of fatty acids are significantly reduced. Given that PC has distinctly long carbon backbones and are entirely saturated may explain the observations noted in the present study. 
Previous research has been able to detect supplementary octacosanol in tissues and/or plasma using radioisotopes (Kabir \& Kimura, 1993) or GC-MS (Menendez et al., 2005). It has been previously suggested that when using radioactivity as a means of quantifying tissue octacosanol, the analysis cannot discriminate between the parent compound and possible metabolites (Lin et al., 2004). Thus, it cannot be discounted that compound degradation could have occurred in the small intestine before absorption or in the liver post absorption. The minimum GC detection limits of PC were assessed in the present study using serial dilutions of pure PC standards dissolved in chloroform. This method produced chromatograms that contained no additional compounds that could interfere with the detection and quantification of the exceeding low levels of PC. However, during the analysis of tissue and plasma, it cannot be excluded that tetracosanol, hexacosanol, heptacosanol, and tricontanol may have eluted from the $\mathrm{GC}$ at identical retention times as were other substances, impeding the identification of the PC of interest. Standard solutions indicated that the retention time for octacosanol was close to that of cholesterol which could impede the quantification octacosanol at very small concentrations. However, a study by Menendez et al. (2005) utilized GC-MS to detect plasma levels of octacosanol from a $10 \mathrm{mg} / \mathrm{kg}$ oral dose of PC supplement in monkeys. Plasma levels of octacosanol peaked at over $400 \mathrm{ng} / \mathrm{ml}$ one hour after ingestion. In the same study when rats were given $60 \mathrm{mg} / \mathrm{kg}$ of PC via a gastric gavage, the maximum concentration of octacosanol found in the plasma and tissues was $30.4 \mathrm{ng} / \mathrm{ml}$ and $68.4 \mathrm{ng} / \mathrm{g}$ respectively. Relative to the dose of PC administered the amount detected in the plasma is small, reinforcing the notion of PC poor absorption. In the Menendez et al. (2005) study, the maximum plasma levels of octacosanol observed in rats was well below the $402.1 \mathrm{ng} / \mathrm{ml}$ detection limit for octacosanol of the present study. GC-MS is a more sensitive means of detection than GC methods. Considering the maximum levels of tissue and plasma octacosanol observed by Menendez et al. (2005) post PC supplementation, it is unlikely that the amount of PC consumed in the present study would have produced plasma and tissue octacosanol concentrations greater than the $402.1 \mathrm{ng} / \mathrm{ml}$ detection limit observed for our GC.

In the present study, hamsters consumed approximately $2 \mathrm{mg}$ of PC supplement per day. Based on the animals' initial body weight and average food intake, this amount was equivalent to approximately $18 \mathrm{mg} / \mathrm{kg} /$ day. Animals receiving the APC1 fortified diet at 10 times the normal amount of PC, corresponded to approximately $183 \mathrm{mg} / \mathrm{kg} / \mathrm{day}$. In humans the maximum 
therapeutic dose of PC is $20 \mathrm{mg} /$ day (Castano et al., 2001b). In a $70 \mathrm{~kg}$ person this amount is equivalent to $0.285 \mathrm{mg} / \mathrm{kg}$. Based on these data, significant alterations in cholesterol metabolism are hard to explain when the low absorption of PC is considered. Further metabolic studies conducted on humans, using reasonable therapeutic doses of $\mathrm{PC}$ and perhaps stable isotopes are required to conclusively define the mechanism concerning the cholesterol-lowering effects of PC.

A greater data range referring to octacosanol recovery was noted between animals receiving $0.275 \mathrm{mg} \mathrm{PC} / \mathrm{g}$ of diet (OPC and APC1) compared to animals receiving $2.75 \mathrm{mg} \mathrm{PC/g}$ diet at 24.7 to $89.7 \%$ and 43.1 to $51.7 \%$ respectively. This observation could be due to fewer animals receiving the higher dose of PC in the diet, resulting in a comparatively smaller number of outliers. It is unknown what happened to the remainder of the ingested octacosanol. Our results show PC was not present in the tissues or plasma of animals receiving PC treatment. It can be assumed that some PC residuals would have remained within the digestive tracts of the animals at the time of euthanization. The amount of residual octacosanol left within the gastrointestinal tract could have been affected by the PC treatment. Studies have shown that as fatty acid chain length increases, the release of cholecystokinin after ingestion decreases (McLaughlin et al., 1999; Jonkers et al., 2000). Cholecystokinin is one the hormones secreted postprandially which facilitate gastric motility (Whitney \& Rolfes, 2002). Since PC has distinctly long carbon backbones and is structurally similar to fatty acids, PC could reduce the postprandial secretion of certain hormones, including cholecystokinin, suppressing gastrointestinal motility. Finally, bacterial degradation of PC could have also affected the amount of octacosanol recovered in the feces. Although we were unable to find studies alluding to colonic fermentation of PC and very long chain acids, microbial breakdown is a metabolic fate of PC that remains to be delineated.

Analysis regarding PC excretion indicated that animals consuming diet containing OPC had significantly higher levels of octacosanol in the feces in relation to those consuming the APC1 formulation (Figure 4.1). Furthermore, a strong trend towards greater octacosanol excretion in the OPC1 group compared to animals receiving 10 times the normal amount of APC1 was also observed. Whether the differences observed between APC1 and OPC regarding octacosanol excretion can be explained by the slight difference in PC composition is yet to be determined. The observation that octacosanol excretion was similar between groups receiving 
normal and high levels of APC1 reinforces the notion that neither OPC nor APC1 instigates a physiological effect. In theory, equivalent intakes would provide APC1 with a longer resident time for absorption, increasing the opportunity for detection. Researchers recently observed that PC significantly increased bile acid excretion in hamsters from 25 to $57 \%$ with diets containing 380,750 and $1500 \mathrm{mg}$ of PC per $\mathrm{kg}$ of diet, resulting in a significant reduction in TC by 15 to $25 \%$ (Ng et al., 2005). Hence, the increased rate of excretion of OPC observed in the present study may have therapeutic significance in connection with reducing levels of circulating cholesterol. However, the PC described by Ng et al. (2005) was obtained as pure octacosanol, triacontanol, and hexacosanol. Therefore, these results cannot be completely extrapolated to answer questions concerning differences observed with respect to cholesterol-lowering efficacy between PC supplements. Nonetheless, the relationship between PC supplementation and bile acid excretion as a potential mechanism for decreasing circulating cholesterol should be further explored.

\subsection{Conclusion}

In summary, results from this study indicate that the Cuban OPC supplements sold in various countries under different brand names have a purity and relative percent PC composition that are consistent with the cited literature referring to the same product. Contrary to previous research, sugarcane-derived APC formulations have a purity and percent composition similar to the OPC mixture. Thus, APC supplements should possess similar cholesterol-lowering properties if the previous conception concerning PC efficacy hinges on the importance of PC purity and composition. In the present study, PC was undetectable in the plasma and tissues of hamsters receiving diets fortified with either the OPC or a sugarcane-derived APC product. Moreover, octacosanol from the APC1 diet were excreted at slower rates. Further research comparing the metabolism of PC from the OPC and APC supplements using therapeutic doses will help determine if mechanistic differences do exist between products. 
Chapter 4 : Manuscript 2

\subsection{Acknowledgments}

The authors would to acknowledge Mary Anne Ryan for her technical assistance and the Advanced Foods and Materials Network for their financial support. We would also like to recognize Degussa Bioactives, Garuda International Inc., and AHD International, LLC for providing alternative sugarcane PC products. 


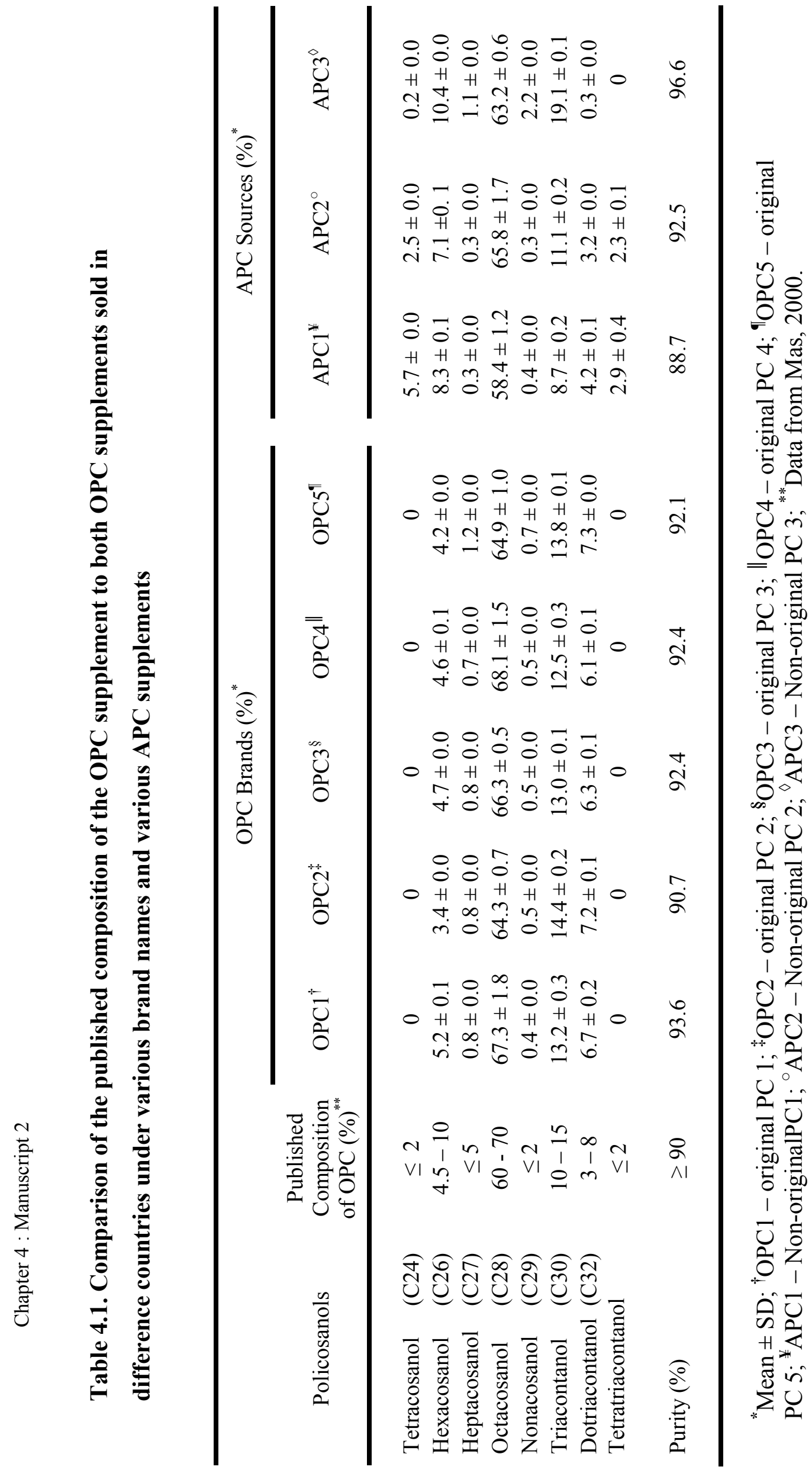


Figure 4.1. Comparison of the ratio of the average 3-day fecal excretion of octacosanol to octacosanol consumption.

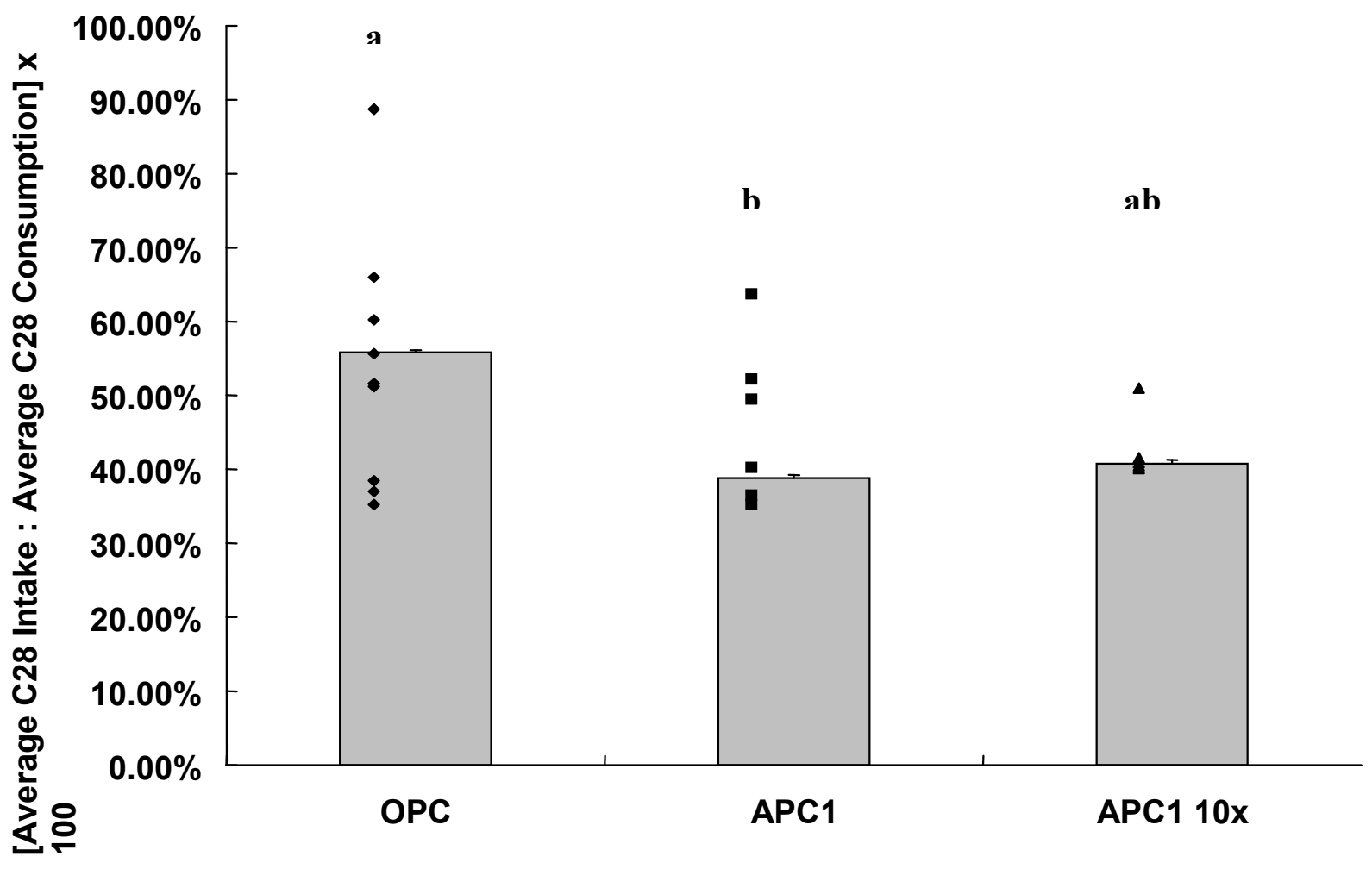

Statistics were performed using arcsine transformation. The reported data have been back- transformed revealing the values obtained before the arcsine was applied. The $p$ value corresponds to data in its transformed state. Original PC (OPC $\bullet$ ), Alternative PC1 (APC1 $\bullet$ ), Alternative PC receiving 10x the normal amount of PC (APC1 10x $\boldsymbol{\Delta}$ ). The $p$ value corresponds to data in its transformed state. Significance $(p<0.05)$ between groups is denoted with different alphabetical labels. 


\section{Bridge 2}

The body of literature on SCP originating from Cuba includes animal and human studies attesting to the cholesterol lowering potential of this natural cholesterol-lowering agent (Varady et al., 2003). Numerous clinical trials have been conducted within various study populations reporting reductions similar to those obtained with statin treatment. Compared to the extensive evidence originating from the Cuban jurisdiction, very few independent human studies exist that have tested the effect of the Cuban product on cholesterol levels. In addition, controversial results from Manuscripts 1 must be reproduced in the context of a human trial in order to determine their application to a target human population. Having shown in Manuscript 2 that no difference exists in the composition and cholesterol-lowering effect between original and alternative mixtures of SCP, a study was undertaken to specifically test the effect of the original product on the lipid profile under controlled compliance conditions in hypercholesterolemic individuals. Unlike previous human clinical trials, the following study was designed as a randomized double-blind crossover where supplementation was done under supervision to reduce inter-individual variation. 


\title{
Chapter 5
}

\section{Manuscript 3: Lack of cholesterol-lowering efficacy of Cuban sugar cane policosanols in hypercholesterolemic persons}

\author{
Amira N. Kassis and Peter J.H. Jones
}

\section{Author affiliation:}

School of Dietetics and Human Nutrition

McGill University, Ste-Anne-de-Bellevue, Montréal, Quebec, H9X 3V9, Canada.

This manuscript has been published in the American Journal of Clinical Nutrition

Kassis AN, Jones PJ. Lack of cholesterol-lowering efficacy of Cuban sugar cane policosanols in hypercholesterolemic persons. Am J Clin Nutr. 2006 Nov;84(5):1003-8.

This project was funded by the Advanced Foods and Materials Network- Network of Centers of Excellence 
Chapter 5: Manuscript 3

\section{Manuscript outline}

5.1. Abstract

5.2. Introduction

5.3. Subjects and methods

5.3.1. Subjects and treatments

5.3.2. Protocol

5.3.3. Plasma lipid analyses

5.3.4. Statistical analyses

5.4. Results

5.4.1. Subjects and drop-out rate

5.4.2. Blood biochemistry and weight changes across the study period

5.4.3. Plasma lipid levels across the study period

5.4.3.1. Total cholesterol responses to treatment

5.4.3.2. LDL-cholesterol responses to treatment

5.4.3.3. HDL-cholesterol responses to treatment

5.4.3.4. Triacylglycerol responses to treatment

5.4.3.5. Effect of sequence, period and body weight change on lipid parameters

5.5. Discussion

5.6. Conclusion

5.7. Acknowledgments 


\subsection{Abstract}

Background: Over 50 studies have reported substantial reductions in plasma lipid levels in response to 2-40 $\mathrm{mg}$ /day of Cuban sugar cane policosanol (SCP) mixtures. However, several animal and human trials conducted outside of Cuba using non-Cuban mixtures have failed to reproduce the efficacy of policosanols observed in earlier studies.

Objectives: Evaluate lipid modulating actions of the originalCuban SCP on plasma lipids in otherwise healthy hypercholesterolemic volunteers.

Design and Methods: Twenty-one volunteers consumed under supervision $10 \mathrm{mg} /$ day of SCP or a placebo incorporated in margarine as an afternoon snack, for a period of 28 days using a randomized, double-blind crossover study design. Subjects maintained their habitual diet and physical activity, and were weighed daily throughout the study period. Blood was collected at days 1, 2, 28 and 29 of the feeding trial and lipid levels were measured.

Results: Body weights did not vary significantly throughout the trial, and did not impact plasma lipid values. No significant difference was observed between treatment and control groups in plasma total, LDL-, HDL-cholesterol and triacylglycerol levels.

Conclusion: Present results show no beneficial effects of Cuban SCP on lipid parameters in hypercholesterolemic individuals and question the clinical usefulness of policosanol mixtures as cholesterol-lowering nutraceutical agents.

Keywords: Double-blind crossover, sugar cane policosanols, cholesterol lowering, lowdensity lipoprotein, hypercholesterolemic individuals 


\subsection{Introduction}

Sugar cane policosanols (SCP) have repeatedly been shown to reduce plasma low density lipoprotein cholesterol (LDL-C), decreasing the risk of cardiovascular incidents (Pons et al., 1992; Pons et al., 1994a; Pons et al., 1994b; Aneiros et al., 1995; Canetti et al., 1995a; Canetti et al., 1995b; Castano, 1995; Castano et al., 1995; Batista et al., 1996; Canetti et al., 1997; Crespo et al., 1997; Ortensi et al., 1997; Castano et al., 1999; Mas et al., 1999; Castano et al., 2000b; Castano et al., 2001a; Castano et al., 2001b; Fernandez et al., 2001; Mas et al., 2001; Mirkin et al., 2001; Castano et al., 2002b; Castano et al., 2002c; Castano et al., 2003a; Castano et al., 2003b; Castano et al., 2004; Mas et al., 2004). Clinical trials of varying durations tested the efficacy of SCP doses ranging from 2 to $40 \mathrm{mg}$ /day and involving subjects from various population groups (Crespo et al., 1997; Ortensi et al., 1997; Castano et al., 2001b; Mirkin et al., 2001; Mas et al., 2004). Results show a reduction in total cholesterol (TC) and LDL-C of 13 to $23 \%$ and 19 to $31 \%$ respectively, as reviewed by Varady et al in 2003 (Varady et al., 2003). The lipidlowering action of policosanols was compared to that of statins in a series of studies showing similar efficacy for the two substances in terms of TC and LDL-C lowering (Ortensi et al., 1997; Fernandez et al., 2001; Castano et al., 2002c; Castano et al., 2003a; Castano et al., 2003b). In a recent meta-analysis comparing lipid-lowering activities of policosanols and plant sterols, a net LDL-C reduction of $24 \%$ was observed in the policosanol treatment groups versus $10 \%$ in the plant sterol groups, as compared to placebo (Chen et al., 2005).

All clinical trials studying the lipid-lowering effect of policosanols have been almost without exception conducted by one group of researchers based in Cuba. However, very recently other investigators have contradicted the positive outcomes of the original research laboratory (Lin et al., 2004; Reiner et al., 2005; Tedeschi-Reiner et al., 2005; Greyling et al., 2006). Although very similar to the original Cuban product, the policosanol mixtures used by these trials differed in source and/or composition of minor alcohols, possibly explaining the discrepancies between findings. Indeed, researchers initially providing data for the remarkable efficacy of sugarcane policosanols have suggested that failure by other groups to observe cholesterol-lowering could be explained by differences in the policosanol ratios in the mixtures tested (Castano et al., 2002b). The 
purpose of the present study was to evaluate the original Cuban mixture of policosanols, as utilized in previous efficacy studies, as ingredients for cholesterol-lowering in moderately hypercholesterolemic individuals.

\subsection{Subjects and methods}

\subsubsection{Subjects and treatments}

Otherwise healthy hypercholesterolemic men and post-menopausal women $(n=21)$ age (40-80 yrs), BMI $\left(23-30 \mathrm{~kg} / \mathrm{m}^{2}\right)$ were recruited for the clinical trial. Volunteers were asked to visit the research unit for two screenings including a blood draw to determine their lipid profile and other health parameters as well as a medical exam. Subjects accepted in the study possessed plasma LDL-C levels ranging from $3.0 \mathrm{mmol} / \mathrm{l}$ to 5.0 $\mathrm{mmol} / \mathrm{l}$ and triacylglycerol levels below $4.0 \mathrm{mmol} / \mathrm{l}$, at screening. Exclusion criteria included history of recent or chronic use of oral hypolipidemic therapy, chronic use of insulin, systemic antibodies, corticosteroids, androgens or phenytoin. Subjects were also excluded if they had experienced a myocardial infarction, coronary artery bypass or other major surgical procedures within the last six months, or reported recent onset of angina, congestive heart failure, inflammatory bowel disease, pancreatitis, or hypothyroidism. Significant pre-existing diseases including cancer, chronic use of laxatives as well as smoking or consumption of more than 2 drinks per day were also considered to be part of the exclusion criteria. Before they were enrolled in the study, subjects were asked to sign a consent form outlining the details of the trial. Policosanols derived from sugar cane wax (Lipex, Dalmer Laboratories, La Havana, Cuba) were purchased in the form of $5 \mathrm{mg}$ pills which were crushed and incorporated in margarine. The composition of the SCP treatment was: $65.6 \%$ octacosanol, $13.4 \%$ triacontanol and $4.5 \%$ hexacosanol (unpublished data). The daily dose of treatment provided $10 \mathrm{mg}$ of policosanols mixed with $10 \mathrm{~g}$ of margarine and was served on a slice of bread.

\subsubsection{Protocol}

The feeding trial was designed as a double-blind crossover, where subjects were randomly assigned to either policosanol or control margarines during two phases of 28 days each, separated by a wash-out phase of the same duration. Subjects were asked to 
maintain their habitual diet and physical activity patterns throughout the study. They were required to visit the clinic daily to consume the treatment as a late afternoon snack under staff supervision at the Mary Emily Clinical Research Centre, in order to ensure absolute compliance. Subjects were asked to abstain from alcohol throughout the two study phases, and caffeinated drinks were restricted to one cup per day. Body weights were recorded daily in order to monitor weight fluctuations throughout the study period. At the beginning and end of each feeding phase, blood draws were scheduled to check for health abnormalities at the onset of the trial and as a result of treatment. All procedures included in the protocol were approved by the ethics committee of the medical faculty of McGill University.

\subsubsection{Plasma lipid analyses}

Fasting blood was drawn from subjects on days 1, 2, 28 and 29 into EDTA tubes, centrifuged at $805 \times \mathrm{g}$ for 20 minutes at $4^{\circ} \mathrm{C}$, then plasma was separated from red blood cells and stored at $-80^{\circ} \mathrm{C}$ for further analysis. Initial lipid values were determined from blood samples taken on days 1 and 2 while end-point values were taken from days 28 and 29. Plasma TC, high density lipoprotein cholesterol (HDL-C), and triacylglycerol (TG) concentrations were analyzed with enzymatic kits, standardized reagents, and standards using a VP Autoanalyzer (Abbott Laboratories, North Chicago, IL, USA). The Friedewald equation was used to calculate LDL-C concentrations (Friedwald 1972).

\subsubsection{Statistical analyses}

The sample size $(\mathrm{n}=21)$ for the clinical trial was calculated to provide an $80 \%$ probability of detecting an anticipated difference in parameters tested of $20 \%$, using a coefficient of variation of $15-20 \%$. Effects of SCP treatment on parameters of interest were compared to control using a one-way ANCOVA for crossover models and subsequent Scheffe's post-hoc test. The effect of sequence, treatment period and weight change were included in the model as covariates to test for potential confounders. Statistical significance was set at a level of $\mathrm{p}<0.05$. 


\subsection{Results}

\subsubsection{Subjects and drop-out rate}

Twenty-two subjects were enrolled in the clinical trial. Baseline characteristics, recorded the first two days of the trial, are outlined in Table 5.1. One subject dropped out because of conflicting schedules. Therefore, twenty-one subjects successfully completed the feeding trial. Most subjects tolerated the afternoon snack well. However, four subjects reported abdominal pain, soft stools and increased frequency of bowel movements. Two of these subjects were consuming the treatment and the other two were receiving the placebo.

\subsubsection{Blood biochemistry and weight changes across the study period}

Blood biochemistry and haematology analyses remained within normal ranges throughout the study period. Initial and final body weights were calculated as average weights of the first and last five days of each phase respectively. These averages are presented in Figure 5.1 and show that subjects maintained a stable weight throughout the study phases. In fact, average final body weights varied by less than $2 \mathrm{~kg}$ from the initial weight during the two treatment periods. Only one participant, subject number 4 , lost 3 $\mathrm{kg}$ during the control phase.

\subsubsection{Plasma lipid levels across the study period}

\subsubsection{Total cholesterol responses to treatment}

Individual TC values across treatment phases are shown in Figure 5.2.1. Original SCP did not significantly affect plasma TC level endpoints or percent changes across each phase, as compared to control. Mean plasma TC was $5.77 \pm 0.21 \mathrm{mmol} / 1$ at the end of the SCP treatment phase, as compared to $5.60 \pm 0.21 \mathrm{mmol} / 1$ in response to placebo $(p=0.25)$. Percent changes from baseline were $(p=0.18) 1.8 \pm 3.0 \%$ and $-4.0 \pm 3.0 \%$ in the $\mathrm{SCP}$ and control groups respectively.

\subsubsection{LDL-cholesterol responses to treatment}

Calculated LDL-C endpoints and percent changes were not significantly affected by SCP supplementation. Individual responses to treatment and control are presented in 
Figure 5.2.2. Mean LDL-C values for SCP treatment and control groups were $(p=0.52)$ $3.69 \pm 0.18 \mathrm{mmol} / 1$ and $3.61 \pm 0.18 \mathrm{mmol} / 1$ respectively. Average LDL-C values were higher than baseline by $4.5 \pm 4.0 \%$ in response to the SCP treatment, and $1.6 \pm 4.0 \%$ lower than baseline in response to control. However differences between groups did not reach significance $(\mathrm{p}=0.28)$.

\subsubsection{HDL-cholesterol responses to treatment}

SCP treatment did not show any significant effect on HDL-C endpoints or percent changes as compared to control. Subject's HDL-C responses to treatments are illustrated in Figure 5.2.3. Mean endpoint values for SCP and control groups were $(p=0.36) 1.37 \pm$ $0.81 \mathrm{mmol} / 1$ and $1.33 \pm 0.81 \mathrm{mmol} / 1$ respectively. Percent changes in HDL-C were $(p=0.19)-2.7 \pm 3.0 \%$ in the SCP group versus $-7.4 \pm 3.0 \%$ in the control group.

\subsubsection{Triacylglycerol responses to treatment}

As for other lipid parameters, no significant effect of SCP was observed on plasma TG endpoints $(p=0.72)$ and percent changes $(p=0.36)$. Individual TG responses to treatment and control are shown in Figure 5.2.4. Mean endpoint values for treatment and control groups were $1.63 \pm 0.24 \mathrm{mmol} / 1$ and $1.59 \pm 0.24 \mathrm{mmol} / 1$ respectively; percent change in the SCP group was $-3.2 \pm 6.0 \%$ from baseline as compared to $-4.3 \pm 6.0 \%$ for the control group.

\subsubsection{Effect of sequence, period and body weight change on lipid parameters}

When study sequence, treatment period and weight changes across periods were included in the statistical model as covariates, no significance was recorded ( $p>0.05)$ as to their effect on plasma mean endpoint values and percent changes of TC, LDL-C, HDL$\mathrm{C}$ and TG levels.

\subsection{Discussion}

Contradicting the previous literature on Cuban SCP, the results of the present study fail to demonstrate that original SCP alters TC, LDL-C, HDL-C or TG levels in otherwise healthy, hypercholesterolemic individuals. Original research on the effect of 
SCP on plasma lipids in humans showed highly promising results in terms of risk reduction for coronary heart disease (Pons et al., 1992; Pons et al., 1994a; Pons et al., 1994b; Aneiros et al., 1995; Canetti et al., 1995a; Canetti et al., 1995b; Castano, 1995; Castano et al., 1995; Batista et al., 1996; Canetti et al., 1997; Crespo et al., 1997; Ortensi et al., 1997; Castano et al., 1999; Mas et al., 1999; Castano et al., 2000b; Castano et al., 2001a; Castano et al., 2001b; Fernandez et al., 2001; Mas et al., 2001; Mirkin et al., 2001; Castano et al., 2002b; Castano et al., 2002c; Castano et al., 2003a; Castano et al., 2003b; Castano et al., 2004; Mas et al., 2004). Tested on numerous study populations and for various durations, SCP treatments proved to be effective regardless of diet control and compliance monitoring. In fact, SCP was often tested in a free-living context where subjects were instructed to follow a National Cholesterol Education Program (NCEP) STEP 1 diet, and given the treatment for consumption under no supervision (Mirkin et al., 2001; Castano et al., 2002c; Castano et al., 2003a; Castano et al., 2004), in some cases once every three months (Mas et al., 2001). Still, doses of SCP as low as $2 \mathrm{mg}$ /day were seen to reduce TC and LDL-C by $14.8 \%$ and $15.6 \%$ respectively (Batista et al., 1996). The design of the present study was such that $10 \mathrm{mg}$ /day of SCP was administered under staff supervision, in order to ensure that subjects were following the study protocol in terms of treatment dose and time of consumption. However, we were not able to replicate the results obtained by previous research on Cuban SCP. On the other hand, the lack of efficacy seen in this trial agrees with the data reported by the few human studies conducted outside the original research laboratory. Lin et al (2004) used $20 \mathrm{mg} / \mathrm{day}$ of a wheat-germ policosanol with similar octacosanol content to the original mixture, for a period of 4 weeks. No benefit was attributed to the effect of policosanols on blood lipids. In two recent randomized double blind placebo controlled crossover trials (Reiner et al., 2005; Tedeschi-Reiner et al., 2005), $10 \mathrm{mg}$ /day of rice policosanols were administered to hypercholesterolemic subjects for a period of 8 weeks. Although the treatment significantly reduced total cholesterol, it did not affect LDL-C, HDL-C and TG. Since the cholesterol-lowering effect of SCP is mainly mediated by a decrease in LDL-cholesterol (Menendez et al., 1996), significant reductions would be expected as can be observed in original policosanol studies. A longer duration of treatment was used in a recent crossover study by Greyling et al. (2006), where participants received $20 \mathrm{mg} /$ day of 
Lesstanol Octa-60 policosanols for a period of 12 weeks. Agreeing with results of a previous animal trial on the same product (Murphy et al., 2004), the authors reported no significant effect on TC and LDL-C in hypercholesterolemic humans. One of the main points of controversy in policosanol research is the appropriateness of the product used in studies yielding negative results. Octacosanol is the major alcohol in policosanol mixtures and was suggested to be the main active component of the treatments (Arruzazabala et al., 2003). Octacosanol content of wheat germ, rice and Lesstanol Octa60 policosanols is similar (Lin et al., 2004) or very close (Reiner et al., 2005; Greyling et $a l ., 2006)$ to the original product. However, mixtures with diverging minor alcohol compositions appear to have different lipid-lowering activities despite their similar octacosanol contents (Castano et al., 2002a). To date, the original policosanol mixture was used in only one clinical trial (Berthold et al., 2006), where the raw material was provided by Dalmer laboratories and the tablets were manufactured in Germany. Results from this recent trial agree with conclusions of external studies finding no effect of policosanols on the lipid profile in hypercholesterolemic patients. Consequently, in the present study, the use of original SCP tablets manufactured in Cuba is of great importance to compare our results to those of original research.

Dissimilarities between original and external research on policosanols include the length of the study period. Unlike external clinical trials with durations ranging from 4 to 12 weeks (Lin et al., 2004; Reiner et al., 2005; Tedeschi-Reiner et al., 2005; Berthold et al., 2006; Greyling et al., 2006), original researchers tested SCP in the context of clinical trials covering 6 weeks to 3 years (Aneiros et al., 1995; Canetti et al., 1995a; Canetti et al., 1995b; Batista et al., 1996; Canetti et al., 1997; Mas et al., 1999; Castano et al., 2001a; Fernandez et al., 2001; Castano et al., 2002c; Castano et al., 2003a; Castano et $a l ., 2003 \mathrm{~b}$ ). However it is important to note that, when interim lipid measurements were performed, SCP efficacy was already manifested by the fourth week of the trial (Fernandez et al., 2001; Castano et al., 2003b). The present study did not reveal any changes in plasma lipids at the end of the 4-week trial, therefore agreeing with external researchers on the inefficacy of policosanols.

The form of administration of a treatment often plays a role in determining its efficacy. Most studies conducted in Cuba used policosanol treatments in the form of a pill 
ingested with a meal. The objective of this study was to evaluate SCP incorporated in a food; therefore, policosanols were mixed in $10 \mathrm{~g}$ of margarine. Fatty matrices, especially margarine have been used as a vehicle for fat soluble substances such as plant sterols (Jones, 1999; Vanstone et al., 2002). Previous literature shows that these matrices resulted in better cholesterol-lowering than others such as low-fat beverages and capsules (Denke, 1995; Varady et al., 2003). Knowing that solubility of policosanols in water is nil (Uribarri et al., 2002), the fatty matrix used was considered appropriate for better mixing and distribution of SCP. Therefore, it is safe to say that the lack of efficacy seen in this study is not likely to be related to the form of administration of policosanols.

Very long chain fatty alcohols (VLCFA) are rather abundant in nature, more precisely in plants and oils that constitute a significant part of the human diet (Hargrove et al., 2004). Specific human intakes of VLCFA are yet to be determined; however estimates of the typical U.S. intake of the wax ester hexacosanoate are reported to be 12$40 \mathrm{mg}$ /day (Hargrove et al., 2004). Octacosanol is found in many common market foods such as rice, wheat, apples, plums, spinach and various nuts and seeds (Hargrove et al., 2004); therefore a diet already providing a high level of octacosanol could potentially mask the effect of a supplementation of this alcohol. Investigating variations in dietary intakes of policosanols in general and octacosanol in particular across populations might determine the involvement of an important factor i.e. the typical octacosanol content of traditional foods consumed. Furthermore, the use of Cuban subjects in original positive research versus a majority of European/North American participants in external negative research draws the attention to the possible effect of genetics and/or dietary habits on policosanol action, potentially making this treatment specific to certain populations.

Despite the extensive research published on the efficacy of SCP, their mechanism of action is still unclear. SCP is believed to be absorbed by the gut (Kabir \& Kimura, 1995), transported to the hepatocytes where it reduces cholesterol biosynthesis by suppressing HMG-CoA reductase (Menendez et al., 1999a; Gamez et al., 2001) and increasing LDL uptake by the cells (Menendez et al., 1997). Radioisotope labelling was used in most studies looking at policosanol absorption and their effect on cholesterol synthesis. In both cases, labelling policosanols could lead to erroneous results due to their degradation in the body into their metabolites (Mannaerts et al., 2000). This would 
suggest that this detection method is non-specific. Also, research has reported poor absorption rates for policosanols in the gut, possibly due to their extreme hydrophobicity (Kabir \& Kimura, 1995; Hargrove et al., 2004; Menendez et al., 2005). In order to understand the mechanism by which a significant amount of SCP can reach the hepatocytes and directly or indirectly induce changes in cholesterol synthesis, more research on the alcohols' bioavailability and their impact on cholesterol kinetics is necessary.

\subsection{Conclusion}

In summary, the present study did not find sugar cane policosanols to be efficacious for the reduction of plasma cholesterol in hypercholesterolemic individuals. Further studies are needed to fully understand their mechanism of action, their metabolism in the body, as well as genetic and dietary factors affecting their action, in order to determine the reasons for controversy in policosanol research.

\subsection{Acknowledgments}

We would like to thank the study participants for their good will and compliance throughout the trial period and the staff of the Mary Emily Clinic for their help with preparing the policosanol treatments. 
Table 5.1. Baseline characteristics of subjects (Mean \pm SEM)

\begin{tabular}{|c|c|c|c|}
\hline Variable & Males $(n=12)$ & Females $(n=9)$ & All $(n=21)$ \\
\hline Age (y) & $54.0 \pm 2.9^{\mathrm{a}}$ & $60.1 \pm 2.6^{\mathrm{a}}$ & $57.8 \pm 2.1$ \\
\hline Weight $(\mathrm{kg})$ & $84.8 \pm 4.9^{\mathrm{a}}$ & $71.1 \pm 3.8^{b}$ & $76.3 \pm 3.2$ \\
\hline BMI $\left(\mathrm{kg} / \mathrm{m}^{2}\right)$ & $26.9 \pm 1.0^{\mathrm{a}}$ & $26.3 \pm 0.8^{\mathrm{a}}$ & $26.5 \pm 0.6$ \\
\hline Total cholesterol (mmol/1) & $5.4 \pm 0.4^{\mathrm{a}}$ & $6.09 \pm 0.3^{\mathrm{a}}$ & $5.8 \pm 0.2$ \\
\hline LDL- cholesterol (mmol/l) & $3.5 \pm 0.3^{\mathrm{a}}$ & $3.8 \pm 0.3^{\mathrm{a}}$ & $3.7 \pm 0.2$ \\
\hline HDL- cholesterol (mmol/l) & $1.2 \pm 0.1^{\mathrm{a}}$ & $1.6 \pm 0.1^{b}$ & $1.4 \pm 0.09$ \\
\hline Triacylglycerols (mmol/l) & $1.5 \pm 0.4^{\mathrm{a}}$ & $1.7 \pm 0.3^{\mathrm{a}}$ & $1.6 \pm 0.2$ \\
\hline
\end{tabular}

Values with different superscript letters are statistically different at $\mathrm{p}<0.05$. Male/Female ratio $=1.3$ 
Figure 5.1. Average initial and final body weights across treatment phases

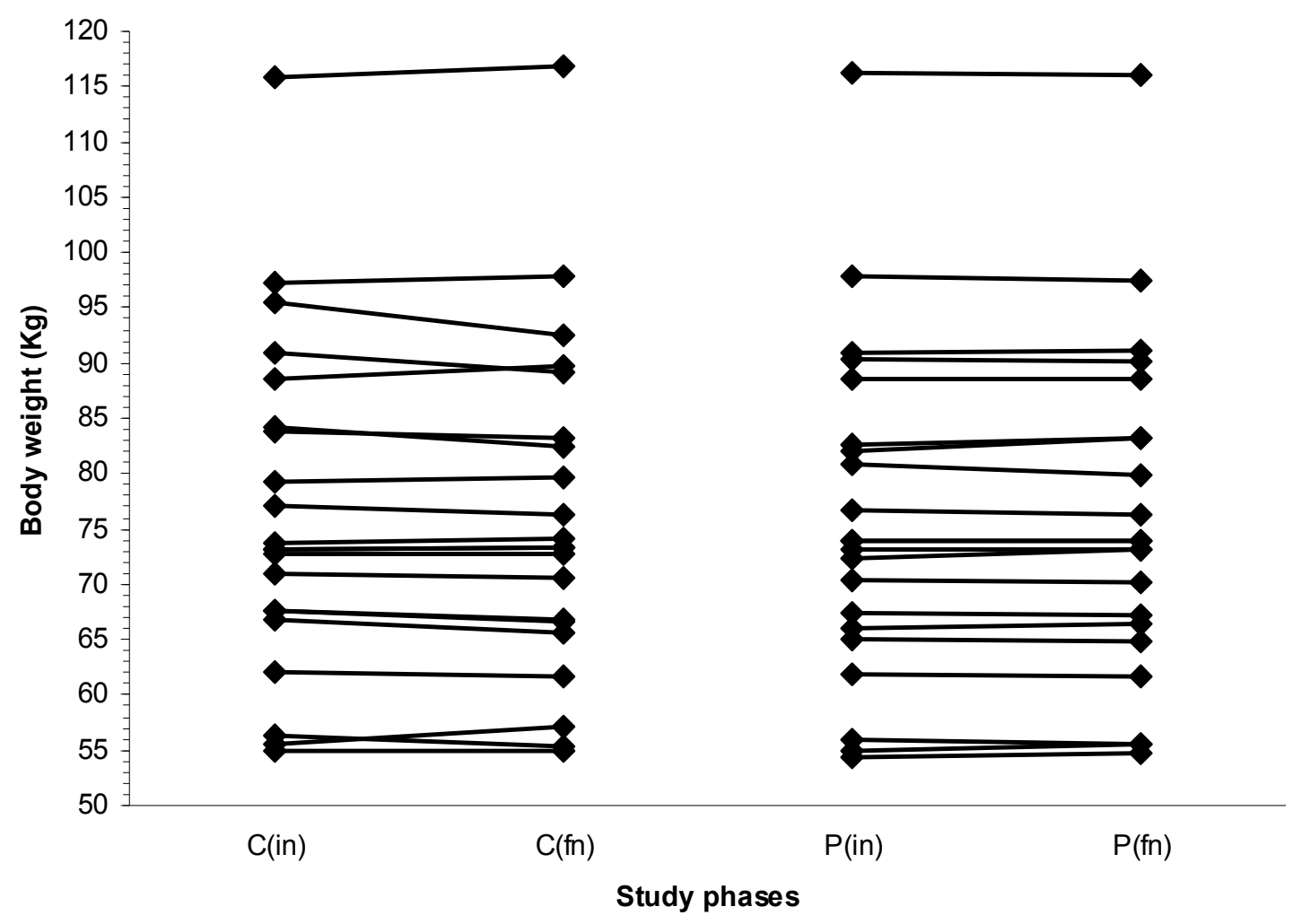

Figure 5.1; $\mathrm{C}(\mathrm{in})$ : Control phase initial value; $\mathrm{C}(\mathrm{fn})$ : Control phase final value; $\mathrm{P}(\mathrm{in})$ :

Policosanol phase initial value; $\mathrm{P}(\mathrm{fn})$ : Policosanol phase final value. 
Figure 5.2. Subjects' plasma lipid endpoints in response to SCP treatment and control

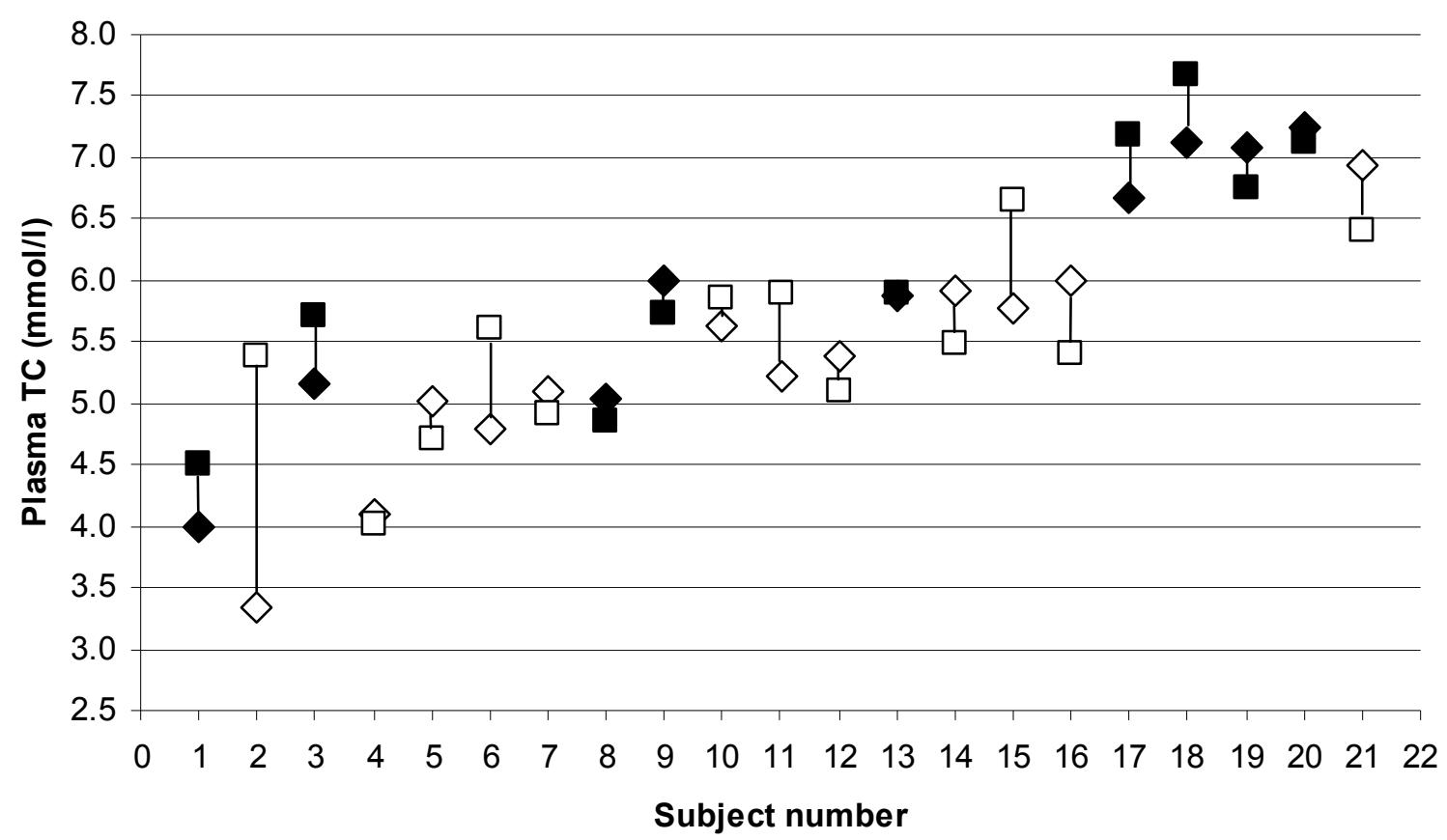

Figure 5.2.1; Individual plasma TC values in response to placebo in men $\diamond$ and women Individual plasma TC values of women in response to SCP treatment in men $\square$ and women $\square(n=21)$ 


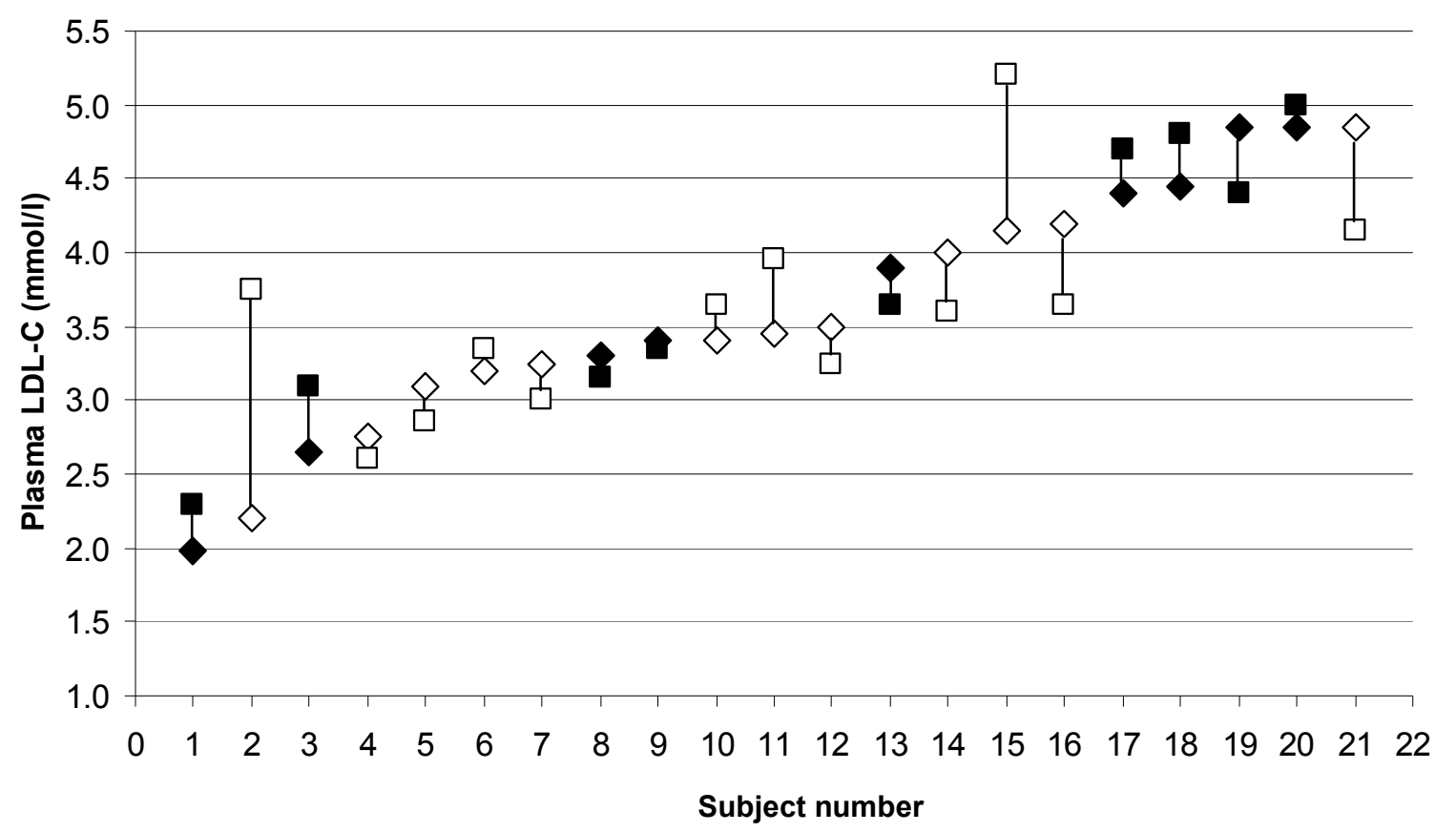

Figure 5.2.2; Individual plasma LDL-C values in response to placebo in men $\diamond$ and women

Individual plasma LDL-C values in response to SCP treatment in men $\square$ and women $(\mathrm{n}=21)$ 


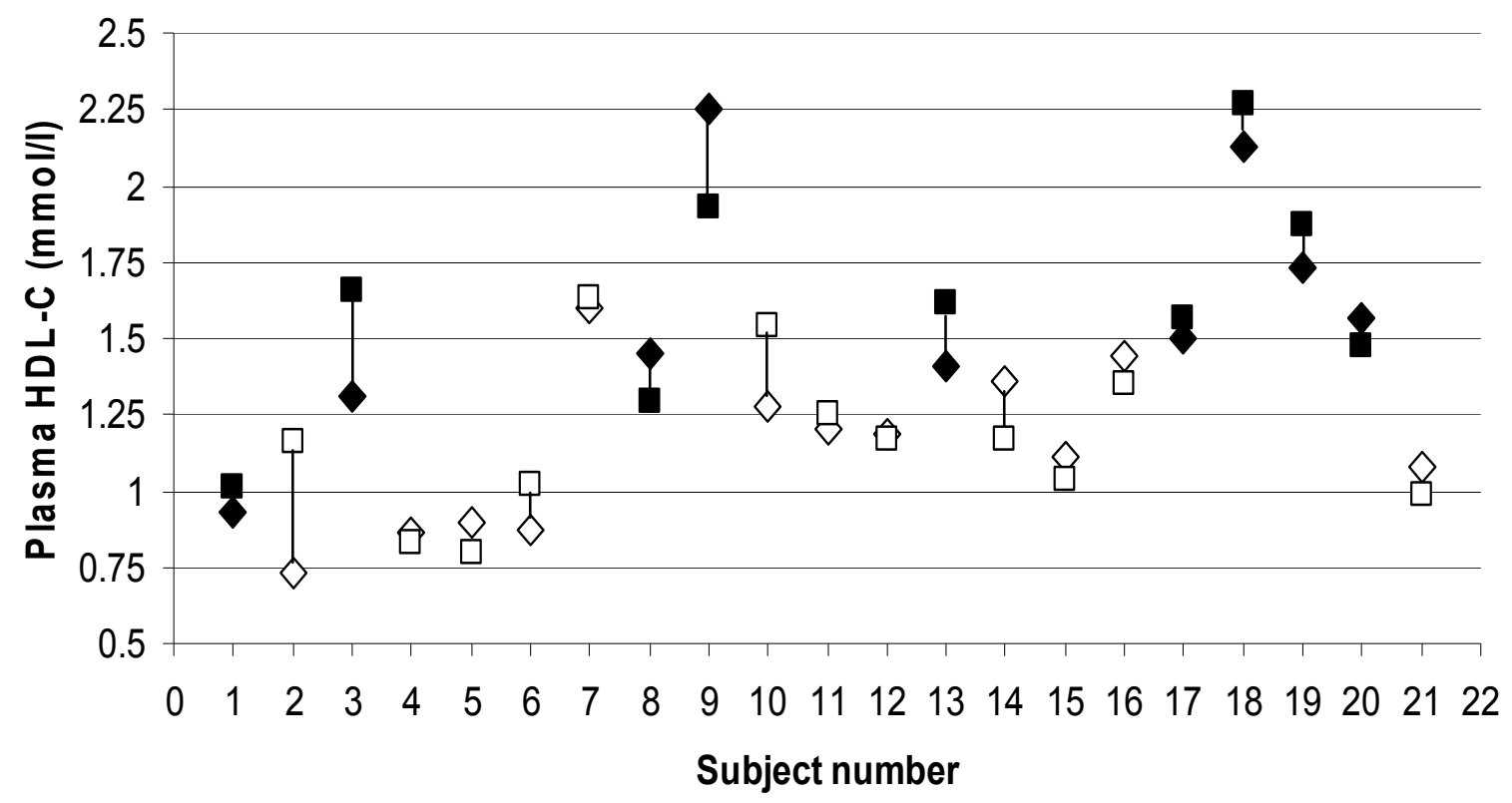

Figure 5.2.3; Individual plasma HDL-C values in response to placebo in men $\diamond$ and women

Individual plasma TC values in response to SCP treatment in men $\square$ and women $(n=21)$ 


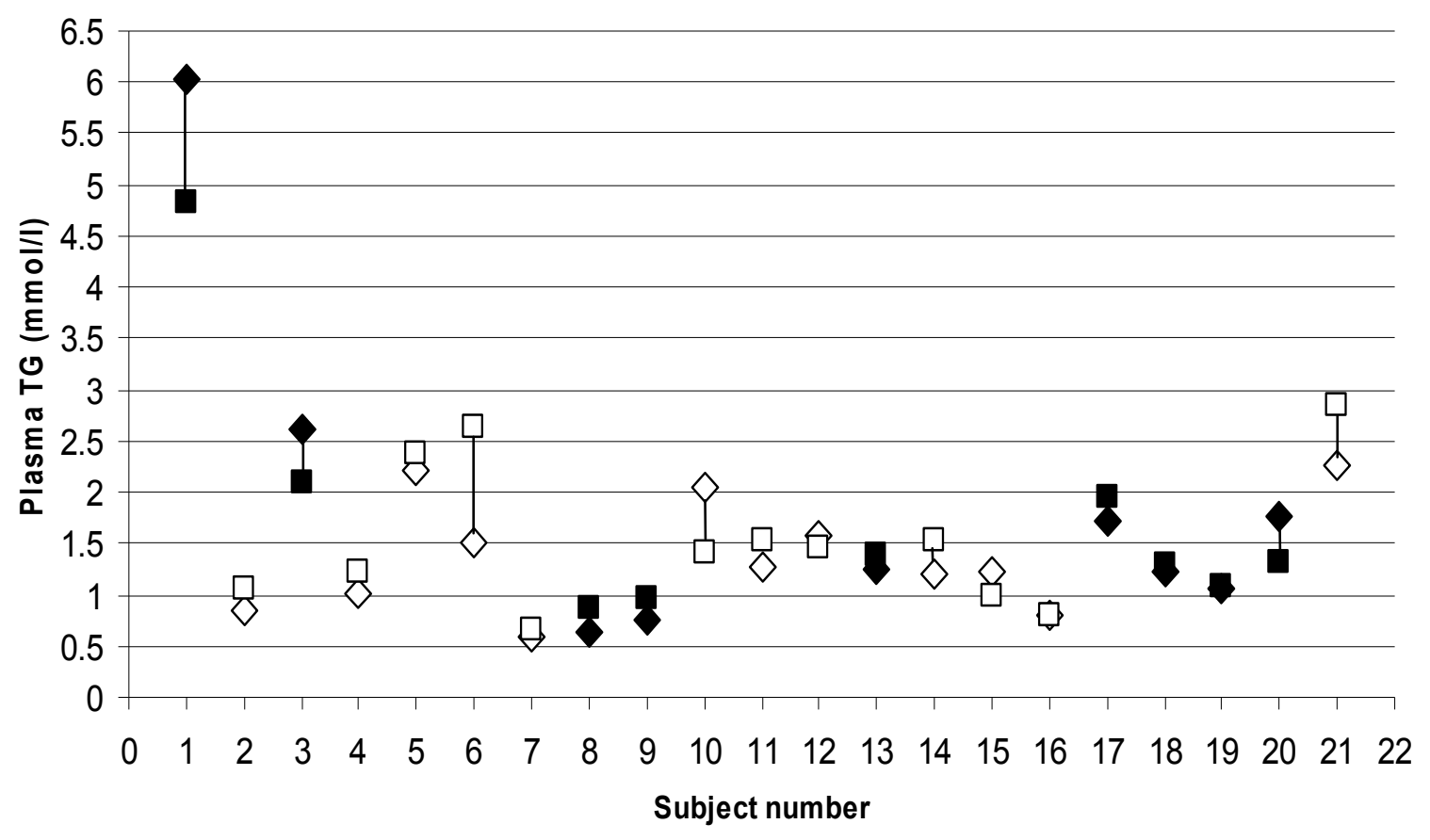

Figure 5.2.4; Individual plasma TG values in response to placebo in men $\diamond$ and women Individual plasma TG values in response to SCP treatment in men $\square$ and women $(n=21)$ 


\section{Bridge 3}

The cholesterol -lowering mechanism of SCP has been investigated in animals and human cells and resulting publications suggest that $\mathrm{SCP}$ is thought to inhibit the activity of HMG-CoA reductase (Menendez et al., 1994; Menendez et al., 1996; Menendez et al., 2001a). Although the exact mechanism is unclear, original research shows an indirect effect at a level between acetate and mevalonate production. Methods used to determine the underlying mechanism of SCP include mainly radioisotope labelling, a methodology which can be strongly criticized for its lack of accuracy. Single-stable isotope ratio methodology is considered to be a reliable procedure for cholesterol absorption and synthesis determination (Wang et al., 2004). Although tritium water incorporation has been used by the original research group on rats and rabbits to measure cholesterol synthesis, no study to date has investigated the effect of SCP on labelled water incorporation in humans. Furthermore, no research is available on the potential inhibitory action of SCP at the level of the intestinal absorption of cholesterol. It is with the aim of clarifying the underlying mechanism behind SCP action in vivo, that the following study was conducted in hypercholesterolemic humans. 


\title{
Chapter 6
}

\section{Manuscript 4: Changes in cholesterol synthesis and absorption following sugar cane policosanol supplementation in hypercholesterolemic individuals}

Amira N. Kassis and Peter J.H. Jones

\author{
Author affiliation: \\ School of Dietetics and Human Nutrition \\ McGill University, Ste-Anne-de-Bellevue, Montréal, Quebec, H9X 3V9, Canada.
}

This manuscript is in press in the Lipids in Health and Disease

This project was funded by the Advanced Foods and Materials Network- Network of Centers of Excellence 


\section{Manuscript outline}

6.1. Abstract

6.2. Introduction

6.3. Subjects and methods

6.3.1. Subjects and treatments

6.3.2. Protocol

6.3.3. Nutrient composition of the diet

6.3.4. Determination of cholesterol absorption

6.3.5. Determination of cholesterol biosynthesis

6.3.6. Statistical analysis

6.4. Results

6.4.1. Compliance and drop-out rate

6.4.2. Plasma lipid levels

6.4.3. Dietary intake throughout the study period

6.4.4. Cholesterol absorption as a result of SCP supplementation

6.4.5. Cholesterol synthesis as a result of SCP supplementation

6.5. Discussion

6.6. Conclusion

6.7. Acknowledgments 


\subsection{Abstract}

Background: Sugar cane policosanols (SCP) have been shown to exert cholesterolmodulating properties in various studies conducted in Cuba through substantial reductions in cholesterol synthesis. Independent research outside Cuba examining changes in cholesterol kinetics in response to SCP is limited to few studies, none of which was able to replicate findings of the original research. Moreover, no data are available on the effect of SCP on cholesterol absorption to date.

Objectives: The present study was undertaken in order to determine effects on cholesterol kinetics, namely synthesis and absorption, within hypercholesterolemic individuals consuming a SCP treatment

Methods: Twenty-one otherwise healthy hypercholesterolemic men and post-menopausal women participated in a randomized double-blind crossover study where they received $10 \mathrm{mg} /$ day of policosanols or a placebo incorporated in margarine as an evening snack for a period of 28 days. The last week of the study phase, subjects were given ${ }^{13} \mathrm{C}$ labelled cholesterol and deuterated water for the measurement of cholesterol absorption and synthesis respectively. Blood was collected on the first two and last five days of the trial. Cholesterol absorption and synthesis were determined by measuring red cell ${ }^{13} \mathrm{C}$ and deuterium enrichment respectively.

Results: The area under the curve for red cell ${ }^{13} \mathrm{C}$ enrichment across 96 hours was not significantly different in the SCP group as compared to control. Similarly, no difference was observed in the fractional rate of cholesterol synthesis over the period of 24 hours between the two treatment groups. Subject body weight remained stable throughout the study and showed no significant correlation with cholesterol absorption or synthesis. The findings of the present study do not agree with the previous research conducted in Cuba concerning the efficacy or mechanism of action of policosanol's cholesterolmodulating properties. 


\subsection{Introduction}

The most commonly used cholesterol-lowering agents contribute to the prevention of cardiovascular disease through their action on cholesterol metabolism, namely absorption and synthesis (Jones et al., 1997; Kosoglou et al., 2005; Davidson \& Robinson, 2006). Sugar cane policosanols (SCP) have been extensively researched within a single jurisdiction in Cuba, purporting efficacy in suppressing cholesterol biosynthesis, resulting in up to $30 \%$ reductions in plasma low density lipoprotein cholesterol (LDL-C). Studies on cholesterol metabolism as a result of SCP supplementation have established that its cholesterol-lowering effect, mainly mediated by a decrease in serum LDL-C levels, was associated with the inhibition of hepatic cholesterol synthesis and the stimulation of receptor-mediated LDL uptake by the liver (Menendez et al., 1994; Menendez et al., 1996; Menendez et al., 1997; Fernandez et al., 2001). Cholesterol synthesis was assessed in vitro by measuring the enrichment of tritiated water into human fibroblasts (Fernandez et al., 2001) and animals in vivo (Menendez et al., 1997). In these studies, synthesis was seen to decrease as a result of SCP treatment.

Unlike statins which inhibit cholesterol biosynthesis by directly affecting the ratelimiting enzyme HMG-CoA reductase, SCP seems to have a different, more complex mechanism of action (Menendez et al., 1994). In studies on human cultured fibroblasts (Menendez et al., 1994; Fernandez et al., 2001) using ${ }^{14} \mathrm{C}$ acetate and ${ }^{14} \mathrm{C}$ mevalonate as tracers, results show that SCP interferes with the cholesterol biosynthesis pathway at a step between acetate and mevalonate production. Since decreased cholesterol biosynthesis is associated with increased LDL-receptor expression in hepatocytes (Basu et al., 1976; Junker \& Davis, 1989), SCP effect on serum LDL-C can be suggested as originating from increased LDL- binding, uptake or degradation, as demonstrated in studies measuring radiolabeled LDL on the surface of human fibroblasts (Menendez et al., 1994).

Although studies conducted in Cuba agree internally concerning effects of SCP on cholesterol biosynthesis, only two studies have been conducted outside the Cuban jurisdiction. The first of these two studies, an in vitro trial, confirmed findings from the original research (Singh et al., 2006). The second trial, conducted on animals, presented conflicting outcomes (Wang et al., 2003). Indeed, the latter contributes to the controversy 
associated with policosanols, showing that cholesterol biosynthesis was not affected in hamsters fed SCP (Wang et al., 2003). We thus recognize that the lipid-lowering mechanism of SCP is not fully understood, especially since other cholesterol-lowering mechanisms such as inhibition of cholesterol intestinal absorption have not been investigated.

The objective of the present study was therefore to examine the effect of SCP in vivo on cholesterol synthesis and absorption in hypercholesterolemic individuals. We hypothesized that there would be no significant change in cholesterol synthesis and/or absorption as a result of SCP supplementation for 28 days.

\subsection{Subjects and methods}

\subsubsection{Subjects and treatments}

Otherwise healthy hypercholesterolemic men and post-menopausal women $(n=21)$ age (40-80 yrs), BMI $\left(23-30 \mathrm{~kg} / \mathrm{m}^{2}\right)$ were recruited for the clinical trial. Volunteers were asked to visit the research unit for two screenings including a blood draw to determine their lipid profile and other health parameters as well as a medical exam. Subjects accepted in the study had plasma LDL-C levels ranging from $3.0 \mathrm{mmol} / 1$ to $5.0 \mathrm{mmol} / 1$ and triacylglycerol levels below $4.0 \mathrm{mmol} / 1$ at screening.

Exclusion criteria included history of recent or chronic use of oral hypolipidemic therapy, chronic use of insulin, systemic antibodies, corticosteroids, androgens or phenytoin. Subjects were also excluded if they had experienced a myocardial infarction, coronary artery bypass or other major surgical procedures within the last six months, or reported recent onset of angina, congestive heart failure, inflammatory bowel disease, pancreatitis, or hypothyroidism. Significant pre-existing diseases including cancer, chronic use of laxatives as well as smoking or consumption of more than 2 drinks per day were also considered to be part of the exclusion criteria. Before they were enrolled in the study, subjects were asked to sign a consent form outlining the details of the trial.

Policosanols derived from sugar cane wax (Lipex, Dalmer Laboratories, La Havana, Cuba) were purchased in the form of $5 \mathrm{mg}$ pills which were crushed and incorporated in margarine. The composition of the SCP treatment was: $65.6 \%$ octacosanol, 13.4\% triacontanol and 4.5\% hexacosanol (Marinangeli et al., 2007). The 
daily dose of treatment provided $10 \mathrm{mg}$ of policosanols mixed with $10 \mathrm{~g}$ of margarine (Becel, Canada) and was served on a slice of bread. $\left[3,4-{ }^{13} \mathrm{C}\right]$ cholesterol and deuterium oxide (99.8 atom percent excess) were obtained from CDN isotopes (Montreal, Canada). $\left[3,4-{ }^{13} \mathrm{C}\right]$ cholesterol was incorporated in margarine (Becel, Canada) by thorough mixing at a concentration of $7.5 \mathrm{mg} / \mathrm{g}$ margarine.

\subsubsection{Protocol}

The study was designed as a randomized double-blind crossover where subjects were assigned to receive the SCP or placebo margarines as a daily evening snack for a period of 28 days. The two treatment phases were separated by a wash out period of the same duration. Study participants were asked to visit the clinic everyday and consume the snacks under staff supervision at the Mary Emily Clinical Research Centre, in order to ensure absolute compliance. Subjects were requested to maintain their habitual diet and physical activity patterns throughout the study and to abstain from alcohol throughout the treatment phases. Caffeinated drinks were restricted to one cup per day and subjects were offered caffeine-free beverages with their snacks. At the beginning and end of each phase, a three-day 24-hour recall was recorded for each subject to ensure that there were no substantial changes in their dietary habits within and between the two phases. Body weights were recorded daily in order to monitor weight fluctuations throughout the study period.

On day 25, subjects were administered $10 \mathrm{~g}$ of margarine spread on a slice of bread providing a dose of $75 \mathrm{mg}$ of $\left[3,4-{ }^{13} \mathrm{C}\right]$ cholesterol. Blood was collected before isotope ingestion (hour 0) and 24, 48, 72 and 96 hours after ingestion in order to measure cholesterol absorption. On day 28 , subjects were asked to drink a dose of $0.7 \mathrm{~g}$ deuterium oxide/kg body water ( $60 \%$ of body weight recorded during the last 5 days). Blood was collected before isotope administration (hour 0 ) and at hour 24 for cholesterol biosynthesis measurements. All procedures included in the protocol were approved by the ethics committee of the medical faculty of McGill University 


\subsubsection{Nutrient composition of the diet}

24-hour recalls of the subjects' food intake for the previous day were recorded at the start and end of each intervention phase. Subjects were advised to write the food items and the exact quantity after each meal to reduce the memory bias during the interview the next day. They were provided with portion models, including cups, plates, bowls, spoons to help them report portions more accurately. Nutrient composition of food items was analyzed using Food Processor version 1.0 and the average macronutrient, cholesterol and dietary fibre content of the diet over three days was computed at the start and end of the phase. Macronutrient intake was then expressed as percent energy.

\subsubsection{Determination of cholesterol absorption}

Blood was collected by venipuncture into 10ml EDTA tubes. Red blood cells (RBCs) were separated from plasma by centrifugation at $15000 \mathrm{rpm}$ for 30 minutes and stored at $-80^{\circ} \mathrm{C}$ for further analysis. Cholesterol absorption was measured using the single-stable isotope-labeled cholesterol tracer approach which was validated against the commonly used dual stable-isotope ratio method and shown to be reliable in the determination of cholesterol intestinal absorption (Wang et al., 2004). The lipid fraction of RBCs was isolated using the modified Folch extraction procedure (Folch et al., 1957). Lipid extracts were analyzed using gas chromatography isotope ratio mass spectromety (GC-IRMS, ThermoFinnigan, Bremen, Germany) where samples were run through a GC unit, a combustion reactor and a mass spectrometer. Briefly, lipids were separated by gas chromatography using a SAC-5 sterol column (Supelco, Milton, Ontario) and isolated cholesterol was directed to the combustion reactor to release $\mathrm{CO}_{2}$ into the MS where ${ }^{13} \mathrm{C}$ enrichment of $\mathrm{CO}_{2}\left(\delta{ }^{13} \mathrm{CO} 2 /{ }^{12} \mathrm{CO}_{2}\right)$ was determined relative to the reference gas. In order to express enrichments relative to Vienna PeeDee Belemnite (V-PDB) limestone, data were transformed using a regression equation between the online and offline IRMS methods. More specifically the equation was developed by running ${ }^{13} \mathrm{C}$ cholesterol samples through the GC-IRMS as well as an offline dual inlet isotope ratio mass spectrometer (SIRA 12, Isomass, Cheshire, UK) providing enrichment values relative to V-PDB limestone. Enrichments were determined for each of hours 0, 24, 48, 72 and 96 
and the area under the curve (AUC) was used to compare cholesterol absorption in the SCP and control groups.

\subsubsection{Determination of cholesterol biosynthesis}

Blood collection was conducted on day 28 (hour 0) and day 29 (hour 24) and lipid extraction was performed using the same methods mentioned for the determination of cholesterol absorption. Cholesterol synthesis was assessed by measuring the rate of deuterium incorporation from body water into $\mathrm{RBC}$ membrane free cholesterol over 24 hours. Lipid extracts from RBCs were separated using GC and isolated cholesterol was introduced into a pyrolysis reactor to release $\mathrm{H}_{2}$ gas. Plasma water samples were run through a high temperature conversion elemental analyzer (TC-EA). Deuterium enrichments for both RBCs and plasma water were measured by IRMS relative to the reference gas. Normalization to Vienna standard mean ocean water (V-SMOW) was performed using a regression equation between the online and offline method (as mentioned above) with data from the offline method expressed relative to V-SMOW. The fractional rate of synthesis (FSR) for cholesterol was then calculated using the following equation:

$\mathrm{FSR}\left(\right.$ pools/day) $=(\delta \mathrm{C} / \delta \mathrm{PW}) \times 0.478^{1}$

Where:

$\delta \mathrm{C}=$ difference in deuterium enrichment between hour 0 and hour 24 for cholesterol $\delta \mathrm{PW}=$ difference in deuterium enrichment between hour 0 and hour 24 for plasma water $0.478=$ ratio of labeled $\mathrm{H}$ atoms replaced by deuterium during in vivo biosynthesis

\subsubsection{Statistical analysis}

The sample size $(\mathrm{n}=21)$ for the clinical trial was calculated to provide an $80 \%$ probability of detecting an anticipated difference in parameters tested of $20 \%$, using a coefficient of variation of 15-20\%. Effects of SCP treatment on cholesterol absorption and synthesis were compared to control using a one-way ANCOVA for crossover models. The effect of sequence, treatment, period, subject and gender were included in the model.

Macronutrient, cholesterol and dietary fibre intakes were compared at the start and end of each phase and between the two interventions using student t-tests. Multiple regressions 
between cholesterol kinetic parameters (AUC and FSR) and nutrient intake as well as age, BMI and LDL levels were performed to identify potential covariates. The model was subsequently adjusted for those covariates. Statistical significance was set at $\mathrm{p}<0.05$.

\subsection{Results}

\subsubsection{Compliance and drop-out rate}

Twenty-two subjects were recruited to take part in the clinical trial and twentyone successfully completed the study. Baseline characteristics of subjects are presented in Table 6.1. The SCP and placebo margarines were well-tolerated. No serious adverse effects were reported throughout the trial with the exception of four subjects reporting gastrointestinal discomfort and more frequent bowel movements. Two of these were in the SCP group while the other two received the control intervention. Body weights remained stable throughout the study periods and blood biochemistry and hematology were within normal ranges.

\subsubsection{Plasma lipid levels}

Plasma lipid levels were measured and results were published in Chapter 5 of the present work (Kassis \& Jones, 2006). Briefly, no significant differences were observed between SCP treatment and control groups in plasma total, LDL, HDL and triglyceride levels.

\subsubsection{Dietary intake throughout the study period}

Macronutrient, cholesterol and dietary fiber intakes throughout the study are presented in Table 6.2. Nutrient content and composition of the subjects' diet were similar at the beginning and end of the two intervention phases. Average caloric and nutrient intakes throughout the SCP intervention were $2005 \pm 127$ calories, $35.7 \%$ from fat (10.9\% saturated), $15.1 \%$ from proteins and $51.1 \%$ from carbohydrates. In comparison, subjects on the control had an intake of $2109 \pm 127$ calories, $35.5 \%$ from fat (11.02\% saturated), 15.3\% from proteins and 50.4\% from carbohydrates. Subjects' dietary cholesterol and fibre intakes were assessed as $141.3 \pm 12.7 \mathrm{mg} /$ day and $19.9 \pm$ $1.59 \mathrm{~g}$ /day respectively during the SCP intervention. Subjects in the control intervention 
had cholesterol and dietary fibre intakes of $120.5 \pm 12.7 \mathrm{mg} /$ day and $20.02 \pm 1.59 \mathrm{~g} /$ day respectively. No differences between groups were noted for all nutrients demonstrating that study participants maintained their dietary habits throughout the study period.

\subsubsection{Cholesterol absorption as a result of SCP supplementation}

Cholesterol ${ }^{13} \mathrm{C}$ enrichments in red blood cells at different time points are illustrated in Figure 6.1. At hour 24, recorded enrichments versus V-DBP were $(p=0.0098)-16.4 \pm 0.43 \%$ and $-16.0 \pm 0.43 \%$ in the SCP and control groups respectively. At hour 48, enrichment values in the two groups were $(p=0.0221)-15.9 \pm$ $0.42 \%$ and $-15.3 \pm 0.43 \%$ respectively. At other time points, no significant difference was observed between SCP and control treatments. When enrichment values were calculated as the difference from baseline (Figure 6.2), SCP supplementation showed no significant effect on this parameter at all time points. Cholesterol absorption rate was determined as the area under the ${ }^{13} \mathrm{C}$ enrichment curve. Calculated AUC for SCP and control is presented in Figure 6.1. Results show that in the SCP group, cholesterol absorption was lower than control by $7.2 \%$, although the difference did not reach significance $(p=0.10)$. The fixed effect of gender on cholesterol absorption was significant ( $\mathrm{p}=0.03$ ), and was manifested by a mean AUC of 513 in female subjects as compared to 362 in males. The effect of diet as determined by multiple regressions was significant $(\mathrm{p}=0.02)$ for caloric intake which was included in the model as a covariate. Adjusted AUC means in the two interventions were not significantly different $(p=0.1)$.

\subsubsection{Cholesterol synthesis as a result of SCP supplementation}

Cholesterol fractional rate of synthesis was $0.0169 \pm 0.001$ pools/ day and 0.0170 \pm 0.001 pools/day in the SCP and control groups respectively with no significant difference between the effects of the two interventions. Results show no difference in FSR between males and females $(p=0.65)$. Daily intake of energy $(p=0.03)$ and energy from saturated fat $(\mathrm{p}=0.03)$ had significant effects on FSR and were included in the model as covariates. The effect of SCP treatment remained non-significant after adjustment for covariates. 


\subsection{Discussion}

The present study is the first human clinical trial to assess the effect of SCP on cholesterol intestinal absorption and biosynthesis using cholesterol isotope labeling methodologies. Our results do not agree with the body of evidence showing that SCP interfere with the biosynthetic pathway of cholesterol in the liver decreasing its levels in the blood in either animals (Menendez et al., 1996; Menendez et al., 1997) or humans (Menendez et al., 1994; Menendez et al., 2001a; Menendez et al., 2001b). The research group originating these claims used rats and rabbits treated with doses of SCP and measured the rate of incorporation of ${ }^{3} \mathrm{H}$ from tritium oxide into hepatic sterols (Menendez et al., 1996; Menendez et al., 1997). The authors of both animal trials report significant reductions of tritium incorporation of up to $50 \%$ as a result of SCP administration to the animals concluding that SCP reduced the rate of cholesterol biosynthesis. However, these findings were challenged by more recent animal data (Wang et al., 2003) using deuterium incorporation into red blood cells in a hamster model treated with SCP and showing no effect on cholesterol biosynthesis as a result of SCP administration. In another trial from the same authors, different doses of policosanols were seen to increase the rate of cholesterol biosynthesis in the animals (Wang et al., 2005). In humans, no studies are available that examine the effect of SCP on cholesterol metabolism in vivo. Rather, in vitro experiments were conducted on human fibroblasts (Menendez et al., 1994; Menendez et al., 2001a; Menendez et al., 2001b) and hepatoma cells (Singh et al., 2006) incubated with increasing doses of policosanols and subjected to the addition of ${ }^{14} \mathrm{C}$-acetate and ${ }^{14} \mathrm{C}$-mevalonate. A clear dose dependant reduction was observed in the incorporation of ${ }^{14} \mathrm{C}$-acetate into cellular cholesterol as a result of SCP treatment (Menendez et al., 1994; Singh et al., 2006). In fact, as policosanol concentration increased, radioactivity incorporated from acetate, but not from mevalonate, decreased significantly reaching an inhibition of $30 \%$ (Singh et al., 2006) and $49.7 \%$ (Menendez et al., 1994) at the $25 \mu \mathrm{g} / \mathrm{ml}$ and $50 \mu \mathrm{g} / \mathrm{ml}$ dose respectively. The authors concluded that policosanols interfered in the cholesterol synthetic pathway at a step between acetate and mevalonate production.

While the evidence from these experiments seems compelling, the method of choice can be strongly criticized when applied with metabolically active compounds, in 
this case policosanols. In a cellular medium, policosanols are metabolized into its corresponding acid and subsequently enter the beta-oxidation pathway (Menendez et al., 2005). A dilution effect can be suggested in this case whereby policosanol-derived acetate contributes to reducing the ratio of ${ }^{14} \mathrm{C} /{ }^{12} \mathrm{C}$-acetate in the medium, resulting in an artificial inhibitory effect of policosanols on cholesterol synthesis. This hypothesis would explain the absence of effect when using ${ }^{14} \mathrm{C}$-mevalonate which is not a product of beta oxidation of policosanols. The present study overcomes the aforementioned limitations by examining the effect of SCP in vivo, using deuterium incorporation into red blood cell cholesterol. This method has been used in numerous human trials and was shown to be an accurate, reliable and safe method for the determination of cholesterol synthesis (Jones et al., 1988; Jones, 1990; Leitch \& Jones, 1993).

The study was designed so that subject compliance to the protocol was ensured by close supervision from the clinical staff and that the SCP treatment was the one used in earlier original research at a dose previously shown to decrease cholesterol levels in humans (Castano et al., 2002c; Castano et al., 2003a; Castano et al., 2004). Moreover, the treatment was administered in the evening because cholesterol synthesis has been shown to reach maximal values at night (Parker et al., 1982; Cella et al., 1995). It remains that no significance was recorded as to the effect of SCP on cholesterol biosynthesis which explains the inefficacy of the treatment in improving the lipid profile of study participants and supports the growing body of evidence originating from independent research groups reporting no change in blood lipids (Lin et al., 2004; Berthold et al., 2006; Cubeddu et al., 2006; Dulin et al., 2006; Greyling et al., 2006; Kassis \& Jones, 2006) .

The poor absorption of SCP in the gut and the increase in synthesis as a result of treatment seen in the Wang et al. study (Wang et al., 2005) generated the interest in determining the effect of SCP on cholesterol absorption in the present study. A mechanism of action similar to that of plant sterols whereby SCP would compete with cholesterol in the gut (Law, 2000), decreasing its absorption and slightly increasing its synthesis, was suggested as a hypothesis in this study. Although absolute ${ }^{13} \mathrm{C}$ enrichments were significantly lower than control in the SCP group at hour 24 and 48, the effect disappeared when levels were adjusted for baseline. Moreover, the AUC for ${ }^{13} \mathrm{C}$ 
enrichments, a better marker for cholesterol absorption was not significantly different across intervention groups. Results thus show no effect of SCP on cholesterol absorption in the gut, and this, independently of the subjects' dietary intake.

Earlier human efficacy studies on SCP did not control dietary intake of participants (Mas et al., 1999; Fernandez et al., 2001; Castano et al., 2002b; Castano et al., 2003a). Subjects were given dietary advice and asked to follow an NCEP step I diet. In the present study, subjects were asked to maintain a stable diet and food intake was recorded thoroughly in order to ensure there were no nutritional differences between the two intervention groups. Results show that nutrient intake remained stable throughout the study phases and between the two groups allowing us to rule out the possibility of a confounding effect of food. Nutrient analyses also showed that caloric intake was negatively related to cholesterol absorption and positively to synthesis. A similar relationship has been reported previously in studies looking at caloric restriction, weight loss and their effect on cholesterol synthesis (Di Buono et al., 1999; Raeini-Sarjaz et al., 2001; Santosa et al., 2007); however few studies have examined the impact of caloric restriction on cholesterol absorption. Although two studies including diabetic subjects observed a negative relationship between cholesterol absorption and caloric intake (Simonen et al., 2000; Simonen et al., 2002), a more recent trial has shown no significant effect of caloric restriction or weight loss on cholesterol absorption (Santosa et al., 2007). Percent energy from saturated fat in the subjects' diet was negatively related to cholesterol synthesis across intervention groups. Saturated fats have long been associated with increased blood cholesterol (Jolliffe, 1961; Grundy \& Denke, 1990); however, their effect on cholesterol metabolism depends on other factors such as concurrent intake of dietary cholesterol and the ratio of polyunsaturated to saturated fats (Salter \& White, 1996). Since dietary intake and fatty acid composition were not controlled in this study, the relationship between saturated fat and cholesterol metabolism remains inconclusive. When energy intake and percent saturated fat were adjusted for in the model as covariates, the SCP treatment remained ineffective in altering parameters of cholesterol metabolism. 


\subsection{Conclusion}

Considering previous independent findings on the inefficacy of SCP (Berthold et al., 2006; Cubeddu et al., 2006; Dulin et al., 2006; Kassis \& Jones, 2006) as well as its poor absorption (Menendez et al., 2005; Marinangeli et al., 2007), the claim that SCP inhibits cholesterol synthesis by an indirect effect on HMG-CoA reductase in peroxisomes is further refuted by the outcomes of the present study. We therefore conclude that this natural extract does not alter cholesterol intestinal absorption and/or hepatic biosynthesis.

\subsection{Acknowledgements:}

We would like to thank the study participants for their good will and compliance throughout the trial period and the staff of the Mary Emily Clinic for their help with preparing the policosanol treatments. 
Table 6.1. Baseline characteristics of subjects (Mean \pm SEM)

\begin{tabular}{|c|c|c|c|}
\hline Variable & Males $(n=12)$ & Females $(n=9)$ & All $(n=21)$ \\
\hline Age (y) & $54.0 \pm 2.9^{\mathrm{a}}$ & $60.1 \pm 2.6^{\mathrm{a}}$ & $57.8 \pm 2.1$ \\
\hline Weight (kg) & $84.8 \pm 4.9^{a}$ & $71.1 \pm 3.8^{b}$ & $76.3 \pm 3.2$ \\
\hline $\operatorname{BMI}\left(\mathrm{kg} / \mathrm{m}^{2}\right)$ & $26.9 \pm 1.0^{\mathrm{a}}$ & $26.3 \pm 0.8^{a}$ & $26.5 \pm 0.6$ \\
\hline Total cholesterol (mmol/l) & $5.4 \pm 0.4^{\mathrm{a}}$ & $6.09 \pm 0.3^{\mathrm{a}}$ & $5.8 \pm 0.2$ \\
\hline LDL- cholesterol (mmol/1) & $3.5 \pm 0.3^{\mathrm{a}}$ & $3.8 \pm 0.3^{\mathrm{a}}$ & $3.7 \pm 0.2$ \\
\hline HDL- cholesterol (mmol/l) & $1.2 \pm 0.1^{\mathrm{a}}$ & $1.6 \pm 0.1^{b}$ & $1.4 \pm 0.09$ \\
\hline Triacylglycerols (mmol/l) & $1.5 \pm 0.4^{\mathrm{a}}$ & $1.7 \pm 0.3^{\mathrm{a}}$ & $1.6 \pm 0.2$ \\
\hline
\end{tabular}


Table 6.2. Diet composition of subjects throughout study period

\begin{tabular}{|c|c|c|c|c|}
\hline & \multicolumn{2}{|c|}{ Control } & \multicolumn{2}{|c|}{ SCP } \\
\hline Average intake /day & Start & End & Start & End \\
\hline Energy (Kcal) & $2126.2 \pm 152.0$ & $2084.5 \pm 148.3$ & $1900.0 \pm 130.2$ & $2109.8 \pm 130.2$ \\
\hline Fat $(g)$ & $87.03 \pm 6.92$ & $80.41 \pm 6.76$ & $72.71 \pm 5.05$ & $83.77 \pm 5.05$ \\
\hline$\%$ energy from fat & $37.15 \pm 1.66$ & $34.26 \pm 1.62$ & $35.33 \pm 1.41$ & $36.15 \pm 1.41$ \\
\hline Saturated fat (g) & $26.37 \pm 2.32$ & $25.09 \pm 2.26$ & $22.97 \pm 1.94$ & $24.84 \pm 1.94$ \\
\hline $\begin{array}{l}\text { Energy from saturated } \\
\text { fat }(\%)\end{array}$ & $11.33 \pm 0.69$ & $10.84 \pm 0.67$ & $11.10 \pm 0.66$ & $10.70 \pm 0.66$ \\
\hline Proteins $(\mathrm{g})$ & $75.90 \pm 6.46$ & $82.47 \pm 6.31$ & $68.52 \pm 4.30$ & $79.23 \pm 4.30$ \\
\hline $\begin{array}{l}\text { Energy from proteins } \\
(\%)\end{array}$ & $14.59 \pm 0.80$ & $15.91 \pm 0.78$ & $14.68 \pm 0.68$ & $15.47 \pm 0.68$ \\
\hline Carbohydrates (g) & $270.29 \pm 22.3$ & $262.59 \pm 21.7$ & $252.82 \pm 22.4$ & $269.33 \pm 22.4$ \\
\hline $\begin{array}{l}\text { Energy from } \\
\text { carbohydrates (\%) }\end{array}$ & $50.28 \pm 1.65$ & $50.36 \pm 1.61$ & $52.11 \pm 1.77$ & $50.11 \pm 1.77$ \\
\hline Cholesterol (mg) & $261.14 \pm 32.8$ & $249.95 \pm 32.0$ & $249.64 \pm 33.9$ & $304.50 \pm 33.9$ \\
\hline Cholesterol (mg/1000 & $123.95 \pm 11.1$ & $116.70 \pm 10.8$ & $130.84 \pm 7.21$ & $151.65 \pm 17.2$ \\
\hline Kcal) & & & & \\
\hline Dietary fibre $(\mathrm{g})$ & $20.98 \pm 1.77$ & $18.82 \pm 1.73$ & $19.34 \pm 1.79$ & $20.71 \pm 1.79$ \\
\hline
\end{tabular}


Figure 6.1. ${ }^{13} \mathrm{C}$ enrichment (Mean $\pm \mathrm{SEM}$ ) of $\mathrm{RBCs}$ after isotope administration in SCP and control groups

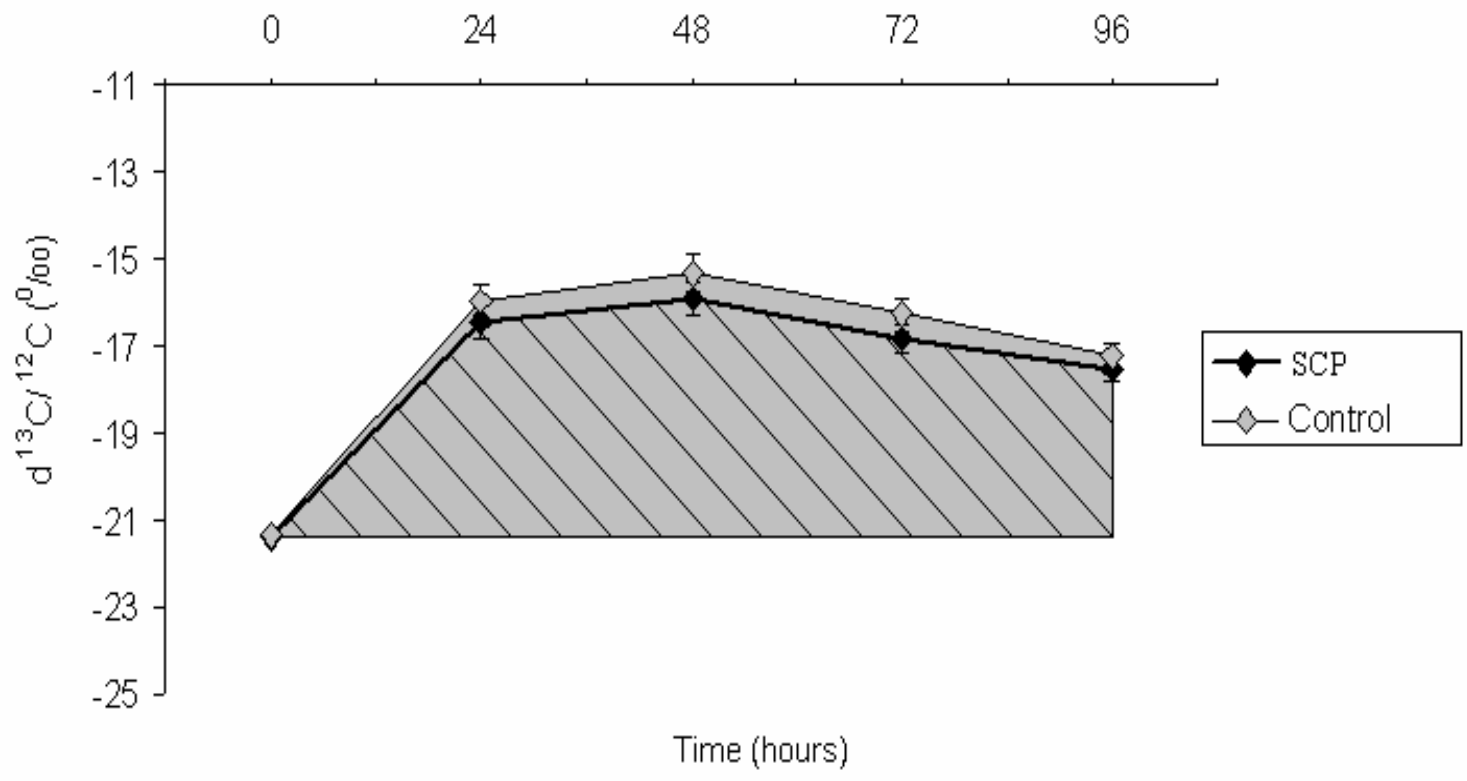

Figure 6.1.; $\mathrm{AUC}_{\mathrm{SCP}}=421.91 ; \mathrm{AUC}_{\text {control }}=454.55$. No significant difference between the two intervention groups. $n=21$ 
Figure 6.2. Difference in ${ }^{13} \mathrm{C}$ enrichment (Mean \pm SEM) between time points and hour 0 as a result of isotope administration in SCP and control groups

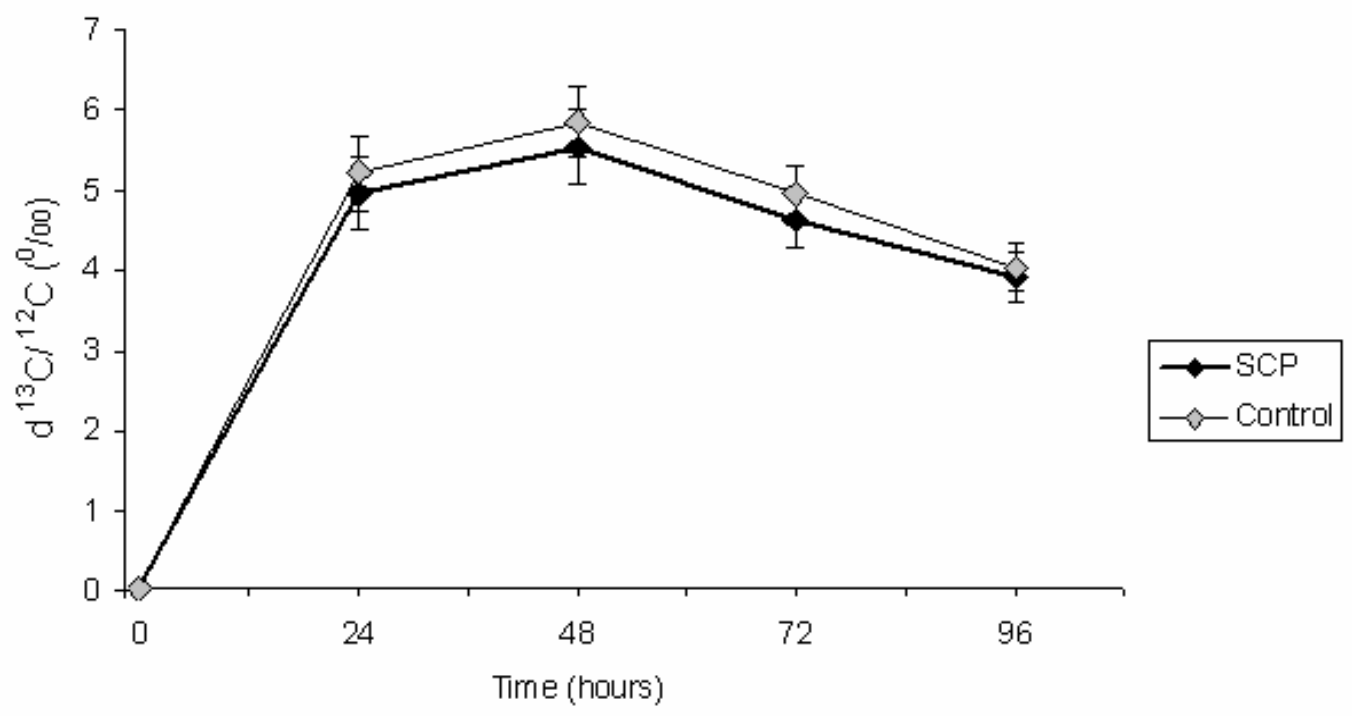

Figure 6.2.; No significant difference between SCP and control groups. $n=21$ 


\section{Bridge 4}

In addition to their cholesterol lowering action, SCP are thought to possess antioxidant properties (Menendez et al., 2000b; Castano et al., 2002c). LDL-oxidation is now known to be a major triggering step in the atherosclerotic process (Williams \& Tabas, 1995), placing antioxidant agents at the forefront of natural health products for the prevention of cardiovascular diseases . Extensive animal and human evidence originating from Cuba indicates reductions in conjugated diene production of up to $38 \%$ in human LDL as a result of treatment with SCP (Menendez et al., 1999b; Menendez et al., 2000b; Menendez et al., 2000c). On the other hand, the only independent study that has looked at the antioxidant power of SCP refuted antioxidant claims, reporting no changes in LDL oxidation in response to SCP ( $\mathrm{Ng}$ et al., 2005). The in vitro nature of the experiment as well as the scarcity of supporting independent data, raises the need for further in vivo research to be undertaken in order to reach a clear conclusion on the effect of SCP on LDL oxidation. Moreover, to date, common methods such as TBARS and conjugated diene assays have been used to measure antioxidant activity of policosanols. However, more reliable procedures should be applied to verify the outcomes of the original research. The following study was carried out as part of the clinical supplementation trial described in Manuscripts $\mathbf{4}$ and $\mathbf{5}$ to assess the effect of SCP on LDL oxidation in hypercholesterolemic adults, using an enzyme immunoassay to quantify oxidized LDL in the plasma as a result of SCP supplementation. 


\title{
Chapter 7
}

\section{Manuscript 5: Lack of effect of Cuban sugar cane policosanols on LDL oxidation in hypercholesterolemic individuals}

\author{
Amira N. Kassis ${ }^{a}$, Stan Kubow ${ }^{a}$ and Peter J.H. Jones ${ }^{b}$ \\ Author affiliation: \\ ${ }^{a}$ School of Dietetics and Human Nutrition, McGill University \\ ${ }^{\mathrm{b}}$ Richardson Centre for Functional Foods and Nutraceuticals, University of Manitoba
}

To be submitted

This project was funded by the Advanced Foods and Materials Network- Network of Centers of Excellence 
Chapter 7: Manuscript 5

Manuscript outline

5.1. Abstract

5.2. Introduction

5.3. Subjects and methods

7.3.1. Subjects and treatments

7.3.2. Protocol

7.3.3. LDL oxidation measurements

7.3.4. Statistical analysis

7.4. Results

7.4.1. Plasma lipid levels

7.4.2. Plasma oxidized LDL

7.5. Discussion

7.6. Conclusion

7.7. Acknowledgments 


\subsection{Abstract}

Background: Sugar cane policosanol (SCP) have been shown to exert antioxidant properties in various studies conducted in Cuba. An independent study since reported no significant effect of SCP consumption on oxidized LDL levels.

Objectives: The main objective of the present study was to examine the effect of Cuban SCP on LDL oxidation using a high-precision capture ELISA procedure in hypercholesterolemic individuals.

Methods: Twenty-one otherwise healthy hypercholesterolemic men and post-menopausal women participated in a randomized double-blind crossover study where they received $10 \mathrm{mg} /$ day of policosanols or a placebo incorporated in margarine as an evening snack for a period of 28 days. Subjects were asked to maintain their usual dietary and exercise habits throughout the duration of the study. Blood was collected on the first and last two days of the trial; plasma was separated and stored at $-80^{\circ} \mathrm{C}$. LDL oxidation was measured from plasma using a solid phase two-site enzyme immunoassay.

Results: Results showed no difference in LDL oxidation between the SCP treatment and placebo at the end of the intervention period. Subject body weight remained stable throughout the study and showed no significant correlation with LDL oxidation levels. Absolute levels of plasma LDL cholesterol had a significant positive effect on plasma concentrations of oxidized LDL.

Conclusions: The findings of the present study do not agree with the original research conducted in Cuba, attributing to policosanols substantial antioxidant properties. Our results align with independent policosanol research questioning the efficacy of this natural extract as a cardioprotective agent. 


\subsection{Introduction}

Atherosclerosis has become the leading cause of death not only in developed (Lefkowitz \& Willerson, 2001) but in developing countries as well (Murray \& Lopez, 1997b; Murray \& Lopez, 1997a), making cardiovascular disease a real global health burden. Several processes have been implicated in early atherogenesis and competing theories on the key triggering events include lipoprotein aggregation, macrophage chemotaxis, foam cell formation and lipoprotein oxidation (Williams \& Tabas, 1995). Atherogenesis has been suggested as triggered by the stimulation of intramural synthesis of molecules promoting lipoprotein retention and subsequent oxidation proposed to be the second most central process in the development of atherosclerosis (Aviram, 1993; Williams \& Tabas, 1995). Sugar cane policosanols (SCP), mixtures of long-chain fatty alcohols, exert cardioprotective properties by reducing the susceptibility of LDL to oxidation as was shown in various studies by a research group based in Cuba (Mas, 2000; Varady et al., 2003; Chen et al., 2005). Animal (Menendez et al., 1999b; Menendez et al., 2000c; Castano et al., 2002c) and human (Menendez et al., 2000c; Castano et al., 2002c) studies demonstrated reductions in lipid and LDL peroxidation as a result of SCP treatment, using markers of oxidative stress such as monitoring of conjugated dienes and measuring thiobarbituric acid reactive substances (TBARS). In animals (Menendez et al., 1999b; Menendez et al., 2000b; Castano et al., 2002c) as well as humans (Menendez et al., 2000c; Castano et al., 2002c), SCP significantly prolonged the lag time, reduced the propagation of conjugated diene generation (Menendez et al., 1999b; Menendez et al., 2000c; Castano et al., 2002c) and decreased levels of TBARS (Menendez et al., 1999b; Menendez et al., 2000b; Menendez et al., 2000c; Castano et al., 2002c). Although independent research outside of Cuba examining the antioxidant power of SCP is limited, the outcomes of a recent study by $\mathrm{Ng}$ et al failed to support previous positive findings, reporting no significant change of oxidation state in LDL from humans treated with a policosanol supplement ( $\mathrm{Ng}$ et al., 2005). Although these negative findings are supported by a growing body of evidence against the claimed cholesterol-modulating properties of Cuban SCP (Berthold et al., 2006; Cubeddu et al., 2006; Dulin et al., 2006; Kassis \& Jones, 2006; Kassis et al., 2006), no independent human trial has examined the 
antioxidant potential of this natural extract. Therefore, the main objective of the present study was to evaluate the antioxidant effect of Cuban SCP in a compliance-controlled clinical feeding trial involving hypercholesterolemic individuals. It was hypothesized that there would be no significant difference in plasma oxidized LDL concentrations between subjects receiving SCP treatment and control.

\subsection{Subjects and methods}

\subsubsection{Subjects and treatments}

Potential participants were screened by phone interview, followed by blood biochemistry analyses to determine their lipid profile, as well as a physical examination conducted by the study physician. Subjects included in the clinical trial $(n=21)$ were otherwise healthy hypercholesterolemic men and postmenopausal women living in the West Island of Montreal. Exclusion criteria included history of recent or chronic use of oral hypolipidemic therapy, chronic use of insulin, systemic antibodies, corticosteroids, androgens or phenytoin. Subjects were also excluded if they had experienced a myocardial infarction, coronary artery bypass or other major surgical procedures within the last six months, or reported recent onset of angina, congestive heart failure, inflammatory bowel disease, pancreatitis, or hypothyroidism. Significant pre-existing diseases including cancer, chronic use of laxatives as well as smoking or consumption of more than 2 drinks per day were also considered to be part of the exclusion criteria. Before they were enrolled in the study, subjects were asked to sign a consent form outlining the details of the trial. The study protocol was approved by the Ethics committee of the medical faculty of McGill University. The SCP treatment administered to subjects during the trial was derived from Cuban SCP manufactured by Dalmer Laboratories (Lipex, Dalmer Laboratories, La Havana, Cuba). SCP composition was $65.6 \%$ octacosanol, $13.4 \%$ triacontanol and $4.5 \%$ hexacosanol calculated as the average composition of the two original mixtures used in the trial (Marinangeli et al., 2007). SCP pills were crushed into a powder and incorporated into margarine (Becel, Canada). Ten grams of the margarine containing $10 \mathrm{mg}$ of SCP were spread on $60 \mathrm{~g}$ of bread and given daily to study participants as an evening snack. 


\subsubsection{Protocol}

The clinical trial was designed as a crossover whereby subjects consumed treatment and control margarines over the course of two 28-day treatment phases. In order to eliminate the carryover effect of treatment, the two phases were separated by a 28-day washout period. During treatment and control phases, subjects were asked to maintain their habitual diets and exercise regimens and visit the research unit everyday to consume the evening snack under staff supervision at the Mary Emily Clinical Research Centre, in order to ensure absolute compliance. Alcohol was prohibited and caffeinated beverages were limited to one cup per day. Body weights were recorded daily in order to monitor weight fluctuations throughout the study period. At the beginning and end of each feeding phase, blood draws were scheduled to check for health abnormalities at the

onset of the trial and as a result of treatment. All procedures included in the protocol were approved by the ethics committee of the medical faculty of McGill University.

\subsubsection{LDL oxidation measurements}

Subjects were scheduled for blood draws at the start and the end of each study phase. Blood was collected from days 1 and 2 as baseline and 28 and 29 to determine endpoint oxidative states. Plasma was separated from red blood cells by centrifugation at $1500 \mathrm{rpm}$ for 20 minutes and was directly stored at $-80{ }^{\circ} \mathrm{C}$ until further analysis. Samples were maintained at $-80{ }^{\circ} \mathrm{C}$ prior to analysis and were analyzed within two years. Oxidized LDL was quantified using a solid phase two-site enzyme immunoassay (Oxidized LDL ELISA, Mercodia, Sweden) with murine monoclonal antibodies mAb-4E6. The plate wells used in this analysis were coated with the capture antibody. The test procedure was applied from the protocol accompanying the assay kit (Mercodia, Sweden). Briefly, plasma samples were thawed then diluted in two steps to reach a final dilution of $1 / 6561$. $25 \mu 1$ of the sample was then added to each coated well and was incubated with assay buffer for 2 hours at room temperature on a plate shaker. This first step insured that oxidized LDL in the sample bound to the solid phase antibodies. The wells were then thoroughly washed to eliminate other plasma components and peroxidase conjugated anti-human apolipoprotein B antibodies were added to recognize the oxidized LDL attached to the solid phase. The labeled antibodies were detected upon the addition of 
3,3',5,5'-tetramethylbenzidine and absorbance at $450 \mathrm{~nm}$ was recorded. A standard curve was drawn for each run using 4 calibrators with different oxidized LDL concentrations as well as a low and a high concentration control. Plasma samples from the two phases belonging to each subject were run on one plate under identical laboratory conditions. Oxidized LDL concentrations of samples run on the same plate were calculated from the absorbance using the equation of a single standard curve.

\subsubsection{Statistical analysis}

The effects of SCP on endpoint LDL oxidation were tested using a one way ANCOVA for crossover models including initial body weight and endpoint LDL cholesterol levels as covariates. Oxidized LDL values did not follow a normal distribution, therefore endpoint values were log transformed to achieve normality and differences in percent changes between groups were analyzed using the Wilcoxon MannWhitney non parametric test. Data were analyzed using the SAS system for Windows version 8.0 (SAS Institute Inc.) and were reported as mean \pm SEM. Statistical significance was set at $\mathrm{p}<0.05$.

\subsection{Results}

Twenty-two subjects were screened and accepted into the study. Twenty-one participants successfully completed the trial. Table 7.1 lists baseline characteristics of participants included in the trial. The policosanol treatment was odorless, colorless and tasteless; therefore subjects were not able to identify the SCP in their snacks. In the control margarine, the SCP powder was substituted by corn starch, the major excipient in the pill, which gave both margarines the same taste and texture. No adverse effects were noted during the study with the exception of four subjects reporting gastrointestinal discomfort and more frequent bowel movements. However, two of these were in the SCP group while the other two received the control intervention. Body weights remained stable throughout the study periods and blood biochemistry and hematology remained within normal ranges. 


\subsubsection{Plasma lipid levels}

Plasma lipid levels were measured and results were published in Chapter 5 of the present work (Kassis \& Jones, 2006). Briefly, no significant differences were observed between SCP treatment and control groups in plasma total, LDL, HDL and triglyceride levels.

\subsubsection{Plasma oxidized $L D L$}

Concentrations of plasma oxidized LDL recorded at the end of the control and treatment phases are illustrated in Figure 7.1. The effect of SCP on LDL oxidation was not significantly different from control $(p=0.76)$. When included in the model, initial body weight did not affect the concentration of oxidized LDL. A multiple regression of oxidized LDL on LDL, HDL cholesterol and triglyceride endpoint levels in the plasma demonstrated a significant effect of LDL cholesterol $(\mathrm{p}=0.03)$ and approaching significance for triglycerides $(\mathrm{p}=0.06)$ on LDL oxidation. Figure 7.2 illustrates a simple regression of oxidized LDL levels on LDL cholesterol concentrations in the study population as a whole. Results show a significant relationship between endpoint values $\left(r^{2}=0.13 ; p=0.023\right)$ and approach significance for percent change values $\left(r^{2}=0.091 ; p=\right.$ 0.061). When oxidized LDL concentrations were expressed relative to LDL levels in the plasma, no effect of treatment was recorded. The effect of treatment phase was significant suggesting that plasma collected during the first phase of the study had higher concentrations of oxidized LDL than the one collected during the second phase. When the model was adjusted for storage time, the effect of treatment phase disappeared and the treatment remained non significant. Percent changes in LDL oxidation after placebo and SCP treatments are reported in Figure 7.3 and showed no difference between the two study margarines in terms of their effect on LDL oxidation $(p=0.61)$.

\subsection{Discussion}

Results of the present randomized controlled study disagree with previous findings claiming efficacy of policosanol in LDL oxidation reduction and agree with 
independent in vitro data showing no antioxidant effect associated with SCP treatment (Ng et al., 2005). Animal (Arruzazabala et al., 1994; Menendez et al., 1999b; Arruzazabala et al., 2000; Menendez et al., 2000b; Castano et al., 2002c) and human (Menendez et al., 2000c; Castano et al., 2002c) trials have previously demonstrated a clear-cut suppression of lipid peroxidation as a result of Cuban SCP treatment. In rabbits with exogenous hypercholesterolemia, SCP exerted a dose-dependant protective effect on atherosclerotic lesions, as 25 and $200 \mathrm{mg} / \mathrm{kg}$ of SCP resulted in less aortic fatty streaks with foam cells of macrophage origin (Arruzazabala et al., 2000). Menendez et al. (Menendez et al., 1999b) conducted a dose response study in rats administering 0, 250 and $500 \mathrm{mg} / \mathrm{kg} /$ day to the animals to evaluate LDL oxidation induced by $\mathrm{CuSO}_{4}$. Continuous monitoring of conjugated dienes and TBARS showed a dose-dependent prolongation of lag time, reduced propagation of conjugated diene generation and reduction of TBARS levels in LDL samples from rats on policosanol treatments. A similar experiment was conducted in healthy volunteers given $5 \mathrm{mg}$ and $10 \mathrm{mg}$ of SCP for a period of 8 weeks. SCP significantly decreased the rate of conjugated diene generation by $38 \%$ and lowered malondialdehyde (MDA) generation by $32 \%$ (Menendez et al., 2000c).The antioxidant action of SCP was also compared to lovastatin in a randomized double-blind study where both substances decreased conjugated diene and TBARS production but only SCP increased the lag time of LDL peroxidation and total plasma antioxidant activity (Castano et al., 2002c).

One limitation to the studies cited above was the methodology used to evaluate LDL-oxidation. Although TBARS is widely used for its simplicity, it is known to be affected by reaction conditions, namely the presence of EDTA (Lapenna et al., 2001). In a comparative study conducted by Carru et al. (Carru et al., 2004), the authors reported that copper oxidation was not a suitable method for the clinical evaluation of LDLoxidation because of the difficulty in standardizing the test and the time needed to run it. More importantly, this procedure did not provide a reliable measurement of conjugated dienes. The method used in the present study was designed to quantify LDL oxidation using monoclonal antibodies directed at antigenic determinants of the oxidized apolipoprotein B molecule, making the test more specific than the assays used by the 
original research groups in terms of discriminating between the sources of oxidation. Since the conversion of native LDL to its oxidized form is a pivotal step in the process of atherogenesis, the choice of the test is crucial for the accurate determination of LDL oxidation. The LDL-ox ELISA method was also chosen in this study because its validity was shown not to be affected by storage time. Circulating oxidized LDL levels have been used as a coronary heart disease marker in previous studies using samples stored for up to 9 years (Holvoet et al., 2001; Hulthe \& Fagerberg, 2002). In addition, the LDL-ox ELISA was seen to be a reliable measure of LDL oxidation in a study examining different storage conditions, namely, freezing samples for up to 3 years (Perman et al., 2004). Plasma samples from our subjects were stored at $-80^{\circ} \mathrm{C}$ up to a maximum of two years; however, our results did show a significant effect of storage time on the concentration of oxidized LDL. Consequently, the data were adjusted for storage time as a covariate. Final results show that the difference between the treatment and control group remained non-significant.

The regression analysis and analysis of covariance performed on the present data revealed clear relationships between LDL levels as a predictor of oxidized LDL concentrations. Our results support previous research (Holvoet et al., 2001; Hulthe \& Fagerberg, 2002) reporting this association. A multivariate analysis of the potential predictors of oxidized LDL concentrations showed that hypercholesterolemia is one of the strongest predictors of the LDL oxidation status (Holvoet et al., 2001). These findings were later confirmed by a study investigating the relationship between oxidized LDL and other markers of atherosclerosis (Hulthe \& Fagerberg, 2002). A significant powerful association was reported between circulating LDL levels and LDL oxidation (Hulthe \& Fagerberg, 2002). Since LDL oxidation clearly varies with circulating LDL levels, the present data were also analyzed using standardized oxidized LDL concentrations relative to plasma LDL values for each subject. After normalization, the data continued to support the lack of antioxidant activity of SCP as shown by the absence of difference between the treatment and control groups.

The mechanisms of action by which SCP could potentially prevent in vivo LDL oxidation are unclear; however, the alcohols were suggested to possibly alter the lipid 
structure of LDL particles by affecting the ratio of unsaturated to saturated fatty acid content (Menendez et al., 2000c; Castano et al., 2002c). Other fatty acids such as oleic acid have been shown to reduce LDL oxidation by altering the fatty acid makeup of lipoproteins as well (Parthasarathy et al., 1990; Bonanome et al., 1992). Absorption rates of oleic acid measured in the lymph reach 58\% in rats (Vistisen et al., 2006). In humans, fecal analysis of labeled oleic acid shows an absorption efficiency of $97.2 \%$ after a bolus administration (Jones et al., 1985a). If policosanols affect the lipid structure in a way similar to oleic acid as suggested by the original research group, it would be expected to have an absorption rate high enough to exert such an effect. Although the bioavailability of Cuban SCP has not been extensively researched, a study from Menendez et al. (Menendez et al., 2005) demonstrated very low concentrations of octacosanol (30.1 $\mathrm{ng} / \mathrm{ml}$ ) and octacosanoic acid in the plasma of hamsters after the administration of a 60 $\mathrm{mg} / \mathrm{kg} \mathrm{SCP}$ dose. These findings were later supported by Marinangeli et al. examining the content of the different alcohols in the plasma and tissues of hamsters on a SCP treatment (Marinangeli et al., 2007). The authors observed no trace of the alcohols in the plasma or tissues of the animals. Taken with the evidence originating from different independent laboratories showing no cholesterol-lowering effect on animals and humans (Berthold et al., 2006; Cubeddu et al., 2006; Dulin et al., 2006; Kassis \& Jones, 2006; Kassis et al., 2006), the authors' conclusion raises the possibility that SCP absorption is not high enough to induce a systemic effect on lipoprotein metabolism and or oxidation status.

\subsection{Conclusion}

In summary, the present investigation examined the claim that SCP plays a preventive role in the development of atherosclerosis by reducing both circulating LDL concentrations and oxidation levels. The findings add to the growing body of evidence raising doubts about the efficacy of SCP as a natural cholesterol-modulating antioxidant therapy. 


\subsection{Acknowledgements}

We would like to thank the study participants for their good will and compliance

throughout the trial period and the staff of the Mary Emily Clinic for their help with preparing the policosanol treatments. 
Table 7.1. Baseline characteristics of subjects (Mean \pm SEM)

$\begin{array}{llll}\text { Variable } & \text { Males }(n=12) & \text { Females }(n=9) & \text { All }(n=21)\end{array}$

\begin{tabular}{|c|c|c|c|}
\hline Age (y) & $54.0 \pm 2.9^{\mathrm{a}}$ & $60.1 \pm 2.6^{\mathrm{a}}$ & $57.8 \pm 2.1$ \\
\hline Weight (kg) & $84.8 \pm 4.9^{\mathrm{a}}$ & $71.1 \pm 3.8^{b}$ & $76.3 \pm 3.2$ \\
\hline BMI $\left(\mathrm{kg} / \mathrm{m}^{2}\right)$ & $26.9 \pm 1.0^{\mathrm{a}}$ & $26.3 \pm 0.8^{a}$ & $26.5 \pm 0.6$ \\
\hline Total cholesterol (mmol/l) & $5.4 \pm 0.4^{\mathrm{a}}$ & $6.09 \pm 0.3^{\mathrm{a}}$ & $5.8 \pm 0.2$ \\
\hline LDL-cholesterol (mmol/1) & $3.5 \pm 0.3^{\mathrm{a}}$ & $3.8 \pm 0.3^{\mathrm{a}}$ & $3.7 \pm 0.2$ \\
\hline HDL-cholesterol (mmol/l) & $1.2 \pm 0.1^{\mathrm{a}}$ & $1.6 \pm 0.1^{b}$ & $1.4 \pm 0.09$ \\
\hline Triglycerides (mmol/l) & $1.5 \pm 0.4^{\mathrm{a}}$ & $1.7 \pm 0.3^{\mathrm{a}}$ & $1.6 \pm 0.2$ \\
\hline
\end{tabular}

Male/female ratio: 1.3 
Figure 7.1. Changes in oxidized LDL across treatment periods

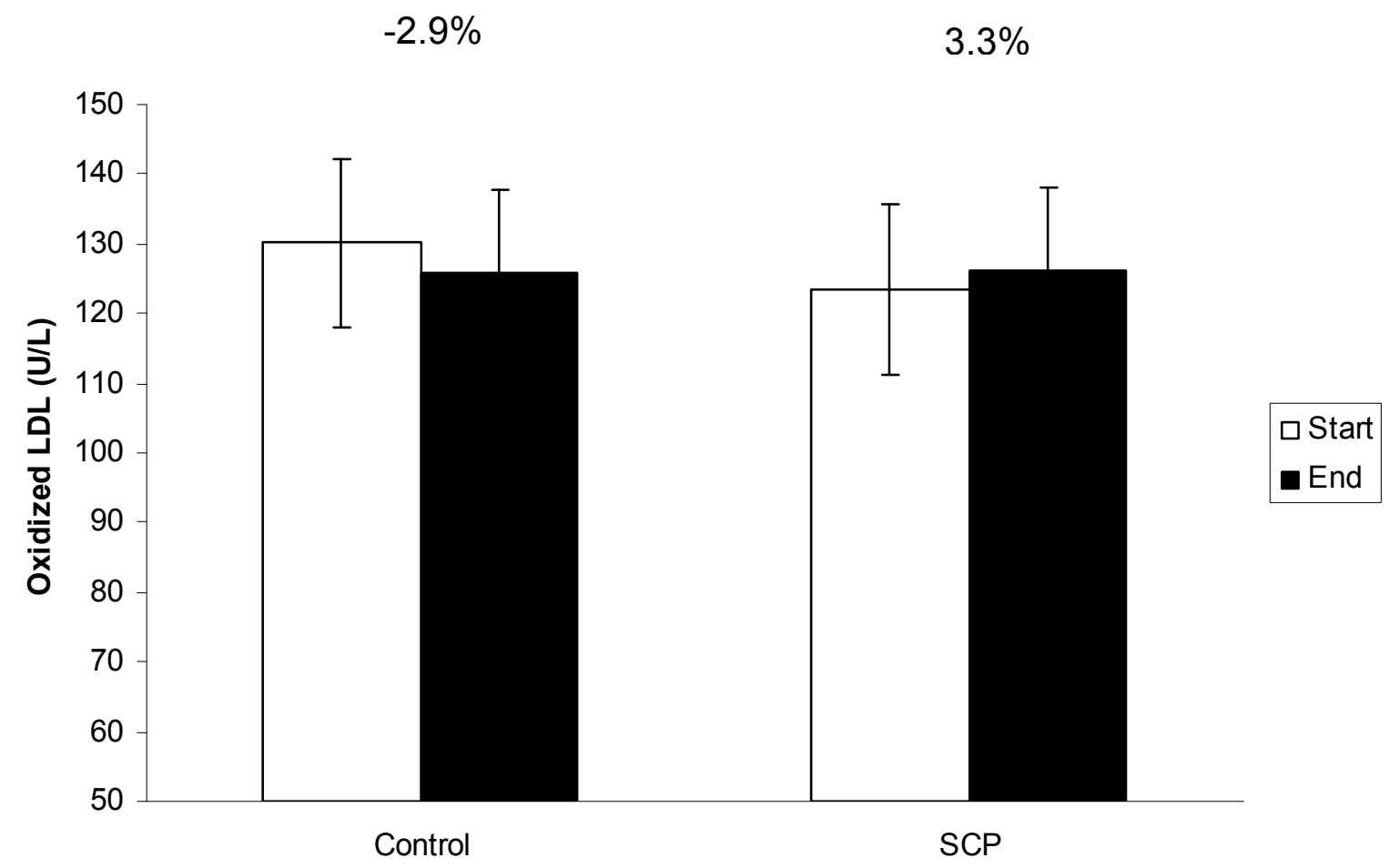

Figure 7.1.; Data reported as Mean $\pm \mathrm{SEM}$. No significant difference recorded between control and SCP groups 
Figure 7.2. Regression of Oxidized LDL concentration and LDL cholesterol levels in plasma

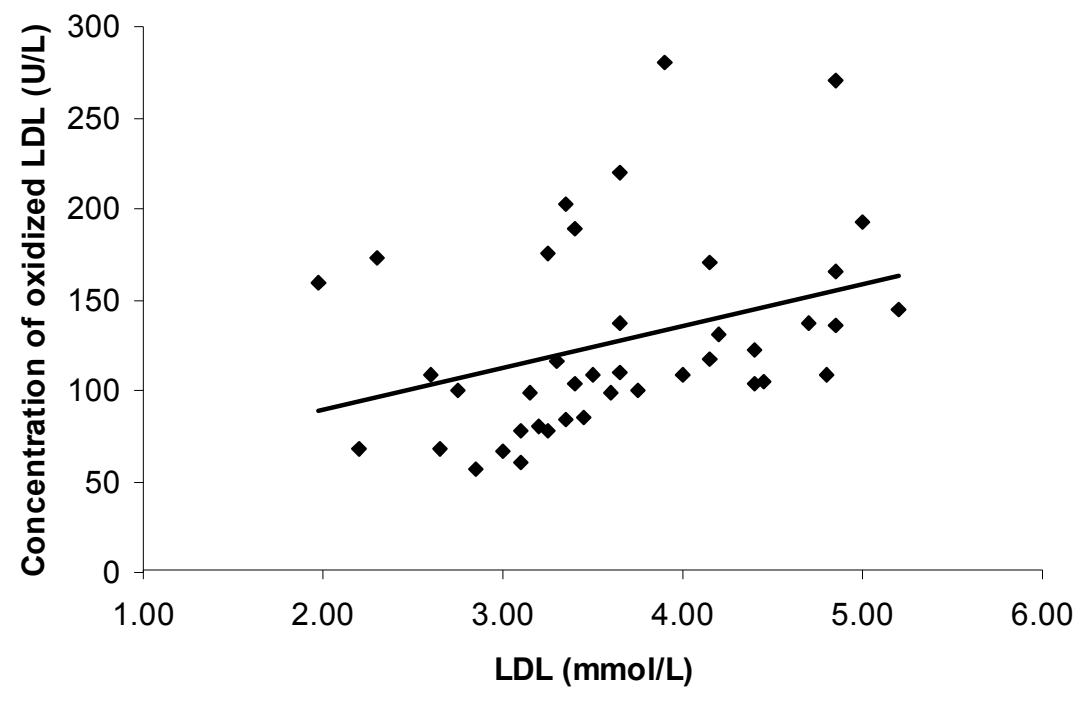

Figure 7.2. ; Regression equation: $y=23.24 x+42.53 . R^{2}=0.0961 ; p=0.02$ 
Figure 7.3. Regression of change in oxidized LDL on change in LDL levels in plasma

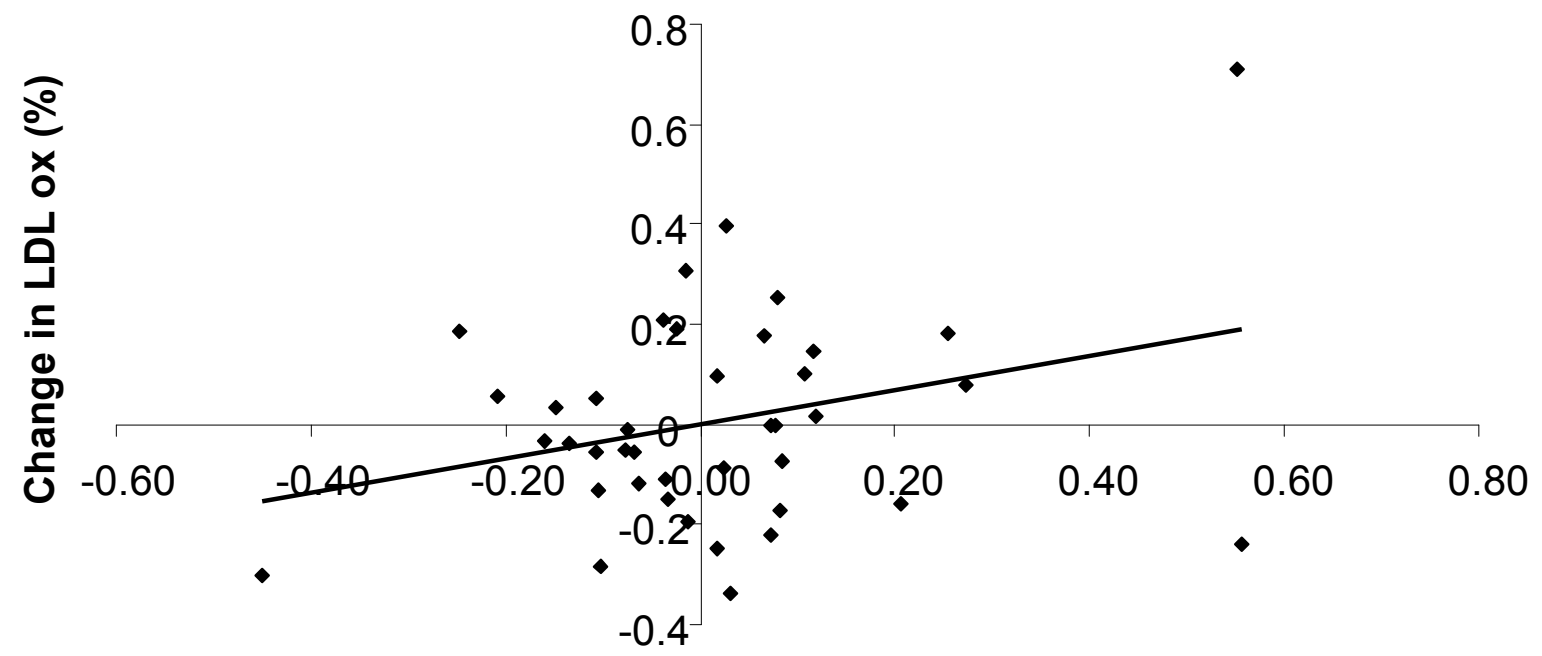

Change in LDL (\%)

Figure 7.3. ; Regression equation: $y=0.34 x+0.012 ; \mathrm{R}^{2}=0.09 ; \mathrm{p}=0.06$ 


\section{Bridge 5}

The policosanol controversy holds its roots in the inability of recent work on policosanols to replicate the extensive body of evidence emanating from Cuban research, holding what seem to be strong and conclusive claims on the substantial health benefits of these natural extracts. The importance of the present work is that it does not only further support recent independent research against claimed befnefits of policosanols but it also provides stronger, more conclusive evidence from both animal and human perspectives. However, based on the original evidence, policosanols are already being widely used, prescribed and marketed independently or as an added value for a range of supplements. This raises a serious concern for the health of consumers who cannot separate valid from weak evidence, and who rely on the scientific community for information on the best health alternative. Therefore, the following editorial was written with the aim of raising awareness within the scientific community on the importance of monitoring research findings in the field of natural health product research, using the policosanol controversy to illustrate the issue around the peer review process and beyond. 


\title{
Chapter 8
}

\section{Manuscript 6: Policosanols lose their lustre}

\author{
Amira N. Kassis ${ }^{a}$, Christopher P.F. Marinangeli ${ }^{a}$ and Peter J.H. Jones ${ }^{b}$

\section{Author affiliation:} \\ ${ }^{a}$ School of Dietetics and Human Nutrition, McGill University \\ ${ }^{\mathrm{b}}$ Richardson Centre for Functional Foods and Nutraceuticals, University of Manitoba
}

To be submitted 
Policosanols, representing mixtures of long chain fatty alcohols ranging from 24 to 34 carbons in length, have been purported to act as effective agents for LDL-cholesterol (LDL-C) lowering in humans. Indeed, over 50 peer reviewed publications attest to 15-30 percent LDL-C reductions with dosages of policosanols ranging from 2- $20 \mathrm{mg}$ per day (Mas et al., 2001; Varady et al., 2003; Chen et al., 2005). However, as pointed out by Varady et al., the original clinical efficacy studies were all performed in a single jurisdiction by essentially a single research consortium. Recently, policosanols have been the subject of controversy since data from independent research groups are beginning to contradict results published by the laboratories originating these claims (Wang et al., 2003; Lin et al., 2004; Murphy et al., 2004; Reiner et al., 2005; Tedeschi-Reiner et al., 2005; Berthold et al., 2006; Greyling et al., 2006; Kassis \& Jones, 2006). In fact, several animal (Wang et al., 2003; Murphy et al., 2004) and human (Lin et al., 2004; Reiner et al., 2005; Tedeschi-Reiner et al., 2005; Berthold et al., 2006; Greyling et al., 2006; Kassis \& Jones, 2006) trials exist from outside the original jurisdiction which fail to support policosanol action. The more recent human efficacy trials have been conducted in Europe, South Africa and North America; ie. over disparate geographic locations, by independent and non-partisan research groups. Although the validity of the more recent studies showing no effect could be questioned initially due to confounders in study design including source and composition of the specific policosanol material utilized, the two most recently completed clinical trials tested the effects of 10-80 $\mathrm{mg}$ of original authentic Cuban sugar cane policosanols (SCP) provided as raw material (Berthold et al., 2006) or in manufactured form (Kassis \& Jones, 2006).

SCP were suggested to reduce plasma LDL-C by suppressing HMG-CoA reductase activity and increasing LDL receptor uptake by cells (Menendez et al., 1997; Menendez et al., 1999; Menendez et al., 2001). In vitro studies conducted by Cuban laboratories have shown that SCP suppress cholesterol biosynthesis by measuring ${ }^{14} \mathrm{C}$ acetate incorporation (Menendez et al., 1994; Menendez et al., 1996; Menendez et al., 2001a), results which were later replicated by an independent laboratory using the same method (Singh et al., 2006). While the evidence from these experiments seems compelling, the method of choice can be strongly criticized due to its inappropriate application with metabolically active compounds such as policosanols. A dilution effect potentially caused by policosanol 
metabolism can mistakenly be interpreted as decreasing ${ }^{14} \mathrm{C}$ acetate incorporation and thus, cholesterol synthesis. In fact, this hypothesis is clearly supported by the newer independent human studies showing that policosanols from authentic or other sources have no true effect on total and LDL cholesterol in hypercholesterolemic humans (Berthold et al., 2006; Greyling et al., 2006; Kassis \& Jones, 2006).Considering that policosanol composition of different alternative sources proved to be similar or very close to the authentic Cuban mixture (Lin et al., 2004; Greyling et al., 2006), reasons for the failure of animal and clinical trials to show efficacy are not obvious. It is possible that genetic characteristics in Cubans may render this population more sensitive to the actions of policosanols compared to other cultural groups; however, no such observation has been previously reported for altered sensitivity to any other natural health product by specific ethnic groups. Another possible explanation for failure of more recent trials to observe lipid-lowering actions of policosanols is that a critical threshold of intake exists for their efficacy beyond which further addition of these agents to diet are ineffective. Possibly, Cuban policosanol intakes may be on average marginal compared with other ethnic and cultural groups. However, it would still be expected that a subset of subjects would respond to policosanol treatment. Subset responsiveness has not been evident in the more recent studies conducted outside of Cuba.

After scrutiny of the totality of existing evidence, one is led to conclude that policosanols have no usefulness in lipid lowering. As such, commercial interests promoting products containing these policosanols ought to be encouraged to re-evaluate whether they should continue marketing such entities. The disparity in data generated by these more recent studies, in the face of the overwhelming number of initial positive studies, raises three important issues.

First, the process of review for publication of scientific studies promoting commercial causes, compared with those which are curiosity driven, ought to be more rigorous. In the case of policosanols, there has been clear opportunity for corporate gain by the private sector interests in demonstrating their efficacy. Moreover, industry scientists working with policosanols have been uncooperative in responding to collaborative invitations by several non-Cuban scientists. It would be expected that promulgators of the original policosanol efficacy papers would welcome the opportunity 
to prove the scientific merit of these bioactives. To avoid repeating a similar pattern of events for other nutraceuticals, it may be necessary to invoke a system of more careful scrutiny of primary scientific data within manuscripts submitted for scientific review, particularly if originating from authors with potential conflict of interest. This notion is reinforced by the recent history of other transgressions in the nutrition arena which have resulted in fabricated data and invalid scientific conclusions.

Second, there is a need for heightened vigilance within our community of scientists, the reputation which stands to suffer as a result of activities misrepresenting scientific truths. As with all societal systems, in science there are inevitably those who choose to break rules and ethical codes for the sake of personal or financial gain. Researchers evaluating grant applications as well as manuscripts for journals should adopt a "neighborhood watch" mentality in identifying potential transgressions before infiltration of false concepts occurs to a level of widespread acceptance. Whether such monitoring is conducted through unofficial channels such as reporting suspicious activity to an individual's institute, or whether a more organized watch-dog organization is required to probe allegations of unethical conduct, is arguable. The limited capacity for policing unethical conduct among scientists by institutional REBs is a major concern; it is likely time to commit more resources to ensuring that unscrupulous actions by researchers can be identified and dealt with so as to avoid systematic transgressions as may be occurring.

Last, events such as the policosanol saga are harmful to acceptance by health professionals and the public of the natural health product category as a whole. At present, medical professional buy-in to the natural health product sector is marginal at best as a result of the number of products available for sale without scientific backup. Advent of Natural Health Product legislation and policies in Canada has served to assist in improving the reputation of nutraceuticals. Disclosure of widespread misrepresentation of policosanols, when major pharmaceutical companies presently carry this material as part of their repertoire, will only further disenfranchise public opinion and support for the natural health product industry. This potential loss of public confidence argues even more strongly for a system of increased regulation and scrutiny at the level of evaluation by 
scientific journals of scientific data which serve the commercial gains of the groups responsible for these data.

Although the final verdict regarding policosanol efficacy will require additional studies to be performed internationally, it is becoming overwhelmingly apparent that a substantial gap in understanding exists between more recent literature and the original data purporting the original discovery of policosanol efficacy in the early 1990s. The eventual dismissal of policosanols as effective cholesterol-lowering agents will hold negative connotations for scientists in the nutraceutical and natural health product arenas. However, increasing scrutiny on behalf of scientific reviewers, relying on a more heterogeneous body of evidence for product approval and marketing and ensuring product quality before it reaches the consumer, can result in positive lessons learned. 


\section{Chapter 9}

\section{Discussion and conclusions}

The present work is an evaluation of the cardioprotective potential of sugar cane policosanols (SCP), a natural health product repeatedly shown to decrease the risk of cardiovascular incidents in Cuban research (Chen et al., 2005). Because atherosclerosis is such a major cause of mortality in industrialized countries (Lefkowitz \& Willerson, 2001), natural alternatives to statin drugs have attracted substantial interest in health research due to the increasing wariness of the public towards statins. SCP have been reported to play a protective role on 2 levels, first, decreasing cholesterol hepatic synthesis and secondly reducing LDL oxidation. We have tested the effect of SCP on both these levels in the present thesis project in order to provide independent data acquired and analyzed outside Cuba, therefore increasing the heterogeneity of the evidence on SCP. In addition, this is the first project to study the effect of policosanols on cholesterol kinetics using stable isotope ratio methodology considered accurate and reliable compared to methods used in the original Cuban literature. Similarly, no in vivo independent data exists concerning the antioxidant capacity of SCP. Our results, like other independent research outcomes, show no change in cardiovascular risk markers, namely, LDL-cholesterol levels and oxidized LDL concentrations in plasma.

The main source of controversy in policosanol research is the difference in design of original versus independent studies. First and foremost, the heterogeneity of policosanol sources in the independent literature makes a fair comparison of research results difficult if not impossible, knowing that Cuban researchers only tested their own Cuban mixtures attributing their efficacy to the unicity of their composition. Secondly, Cuban SCP were investigated by a single research group, most trials and analyses conducted in the Center for Natural Products, Center for Scientific Research located in La Havana, Cuba. All SCP products were manufactured and provided by the Dalmer laboratories located in La Havana, Cuba. Such close research collaborations with an industry are typically at risk for conflict of interest which can reduce the validity of study findings. Even though it is difficult to determine whether financial gains influence 
investigators and the accuracy of their research outcomes, it is always best to avoid introducing motives which could distract researchers from conducting sound ethical research (Thompson \& Montarella, 1993). This fact further supports the need for independent data to validate the Cuban body of evidence. Finally, methods used by the original group are not optimal for the assessment of cholesterol synthesis in response to SCP treatments. As mentioned earlier in the present work, radioactive isotope methods do not provide an accurate quantification of the compound of interest, both for the assessment of policosanol absorption and their effect on cholesterol synthesis. The mentioned limitations also reduce the replicability of the Cuban evidence and might explain the lack of efficacy seen in independent studies. On the other hand they raise the need to push policosanol research further into reevaluating their efficacy using improved designs and techniques. For this reason, the present project was undertaken with the aim of evaluating the cardioprotective properties of SCP while overcoming the limitations of independent research such as the use of alternative policosanol mixtures and the lack of compliance control.

Prior to the start of the present work, independent research on policosanols was criticized by the original research group, mainly because of the use of alternative policosanol preparations with different alcohol compositions (Castano et al., 2002b). The compositions of different policosanol mixtures have been analysed as part of the present thesis and is presented in Manuscript 2. Contrary to the claims made by the original Cuban researchers, all mixtures tested fall within the published range of composition of the original SCP used by Cuban researchers, including the preparation used in the hamster trial described in Manuscripts 1 and 2. These results explain the lack of difference we observed between the alternative and original brands in their effects on the lipid profile of hamsters.

Cuban original research agrees with the present work on the limited intestinal absorption of policosanols. Minute amounts of the alchohols were found in the plasma of animals after supplementation in a study by Menendez et al in 2005. The animal component of the present work showed no trace of policosanols in the plasma and tissues of hamsters, leading to the conclusion that SCP are not absorbed by the body. The possibility that policosanols affect cholesterol metabolism after their conversion into their 
corresponding acids still remains, for we have not looked at the concentration of very long chain fatty acids in the plasma and tissues. Measuring these concentrations in the context of a controlled metabolic study would be a useful complement to the data hereby presented to rule out any other mechanism by which policosanols affect lipid metabolism. Moreover,

Taking hamsters' daily food intake into consideration, we ensured that the dose of policosanols ingested was on average $20 \mathrm{mg} / \mathrm{kg}$ body weight. This dose is deemed appropriate as it falls within the range of doses that have previously been shown to be efficacious in terms of cholesterol lowering, that range being $5-500 \mathrm{mg} / \mathrm{kg}$. On a human scale, this dose would represent a $1400 \mathrm{mg}$ /day for a 70kg adult, more than 100 times the recommended dose for humans. As for the background diet, policosanol treatment is prescribed with a low cholesterol diet in order to avoid potential cholesterol synthesis suppression thought to occur as a result of high dietary cholesterol (Menendez et al., 1997). For this reason, cholesterol content of the animal feed in the present project was decreased in comparison to a previous hamster study showing no cholesterol-lowering linked to policosanol supplementation. The level of cholesterol ( $0.1 \%$ by weight $)$ used in the animal trial can be more easily applied to a human diet than the no-cholesterol high starch casein diet used in the original research. This diet was fed to the animals for a maximal increase in cholesterol synthesis, mimicking endogenous hypercholesterolemia. Knowing that hamsters in the present work ate about $7 \mathrm{~g}$ of feed per day and that their weight was around $100 \mathrm{~g}$, their cholesterol intake from the feed was equivalent to around $400 \mathrm{mg}$ on a human scale, which is considered as an average consumption in a typical North American diet (FDA, 1998). SCP has been shown to be effective in endogenous normocholesterolemia, and synthesis suppressors such as statins have previously been reported to decrease cholesterol levels in the context of a high cholesterol diet. One would therefore expect to see a change in cholesterol synthesis in our case of exogenous hypercholesterolemia. Furthermore, we have assessed the effect of the diet on SCP efficacyin humans, in Manuscript 4 through 24-hour dietary recalls. Results show that, although dietary intakes of energy and saturated fat affect FSR, the lack of SCP efficacy is independent of dietary composition. 
The potential effect of policosanols on cholesterol absorption is another hypothesis tested in the present thesis. It is in fact possible that, given the poor absorption of very long chain fatty alcohols and acids, an inhibitory action at the level of the gut could decrease absorption of dietary cholesterol. A plant sterol like competition with cholesterol for micelle incorporation is one of many mechanisms that could be suggested for cholesterol lowering efficacy of policosanols. On the other hand, assuming that cholesterol synthesis is in fact decreased, an upward compensation at the intestinal absorption level could be a possible explanation for the lack of net effect on cholesterol levels in the plasma. Although this work has not explored the specific mechanisms of action of policosanols on cholesterol intestinal absorption, the net effect on cholesterol absorption was a major endpoint of the human component of the project. As our results show, there was no significant difference noted in overall absorption over the course of 96 hours as compared to placebo.

The discrepancy between results from the Cuban literature and outcomes of the present work could be explained by the potential uniqueness of the Cuban population and / or a difference in composition between the Cuban and North American diets. Genetic influences have previously been observed on fat and cholesterol metabolism (Lee et al., 2001; Pallauda et al., 2001; Ordovas, 2006) and epidemiological studies have often depicted different disease risks in different populations. Heart disease risk is higher among African Americans and Mexican Americans and lower among Asians (Brown et al., 2005; Clark, 2005; Mensah et al., 2005). These differences between populations are usually attributed to a genetic makeup, more or less prone to develop cardiovascular diseases. It thus cannot be ruled out that the Cuban population might have a higher responsiveness to SCP supplementation than the present study population made up mostly of North Americans of European descent. In addition, although one independent negative study was conducted on a South-African population (Greyling et al., 2006), most independent trials included European or North American participants. If the Cuban population is characterized by an altered expression of genes involved in cholesterol synthesis, future independant research on SCP should include more heterogeneous study populations in order to examine the distribution and the effect of certain polymorphisms on SCP efficacy. Examples of genes involved in the regulation of cholesterol synthesis 
include those coding for squalene epoxidase (Sqle), mevalonate kinase (Mvk), and farnesyl diphosphate farnesyl transferase 1 (Fdft1) recently localized (Bonné et al., 2002).

The natural occurrence of policosanols in the diet could also be accountable for the lack of efficacy seen in this and other independent work on policosanols. Policosanols are found in a wide variety of plant foods such as rice, wheat, and apples (Hargrove et al., 2004), therefore it can be argued that a high intake of these foods in the diet may decrease the cholesterol-lowering potential of supplementation. Whether the North American/ European diet contains more policosanol rich foods than the Cuban diet is yet to be determined as there are no food composition records that take account of very long chain fatty acids and alcohols to date. We have assessed subjects' food intake in the present study in order to examine the potential effect of diet. However, this variable could have been better controlled had our design included a fully controlled feeding trial and a food analysis to quantify policosanol content of meals. This shortcoming in our methodology should be taken into account in future research on policosanols.

Although the dietary difference argument is plausible in a human population, it is not supported by the lack of efficacy seen in the present work and other independent research using hamsters and rabbits considered to be among the best animal models to investigate human lipid metabolism. Animal feed is standardized across the literature and differences amongst study diets, if any, are not large enough to modulate the response to policosanol treatments. This point has been mentioned and discussed earlier in the present work (Manuscript 1).

If genetics and dietary habits are considered as potential confounders in the study of SCP efficacy, the claims related to their cholesterol-modulating properties and other cardioprotective actions are not warranted for use to the general public, especially considering the lack of data available on the variability of response across different populations.

Following the start of the present thesis work, over ten independent studies have been published, all of which have shown no significant effect of policosanols on cholesterol levels and oxidative stress. Independent literature made substantial progress by the use of original policosanol material as treatment as well as improving study design 
(Berthold et al., 2006). The growing body of recent evidence refuting policosanol associated claims provides additional support to the outcomes of this work; however, this thesis remains to date the first independent initiative to examine the effect of the original policosanol mixture on cholesterol kinetics using stable isotopes, and LDL-oxidation in humans in a randomized clinical trial ensuring full compliance to the treatment.

In summary, the evaluation of policosanols undertaken in this thesis does not only support previous independent research showing no cholesterol-lowering efficacy, but reinforces the contradicting evidence through the use of stronger study designs, improved assessement methods and increased compliance. While policosanols from all sources are being increasingly prescribed and sold over the counter around the world, the evidence supporting its efficacy is only limited to the Cuban population. Therefore it is mandatory to raise the awareness of the scientific community as well as consumers who already consider policosanols as natural thus healthier alternatives to statin drugs. 
Alcocer L, Fernandez L, Campos E \& Mas R (1999) A comparative study of policosanol Versus acipimox in patients with type II hypercholesterolemia. Int J Tissue React 21, 85-92.

Aleman CL, Mas Ferreiro, R., Noa Puig, M., Rodeiro Guerra, I., Hernandez Ortega, C. \& Capote, A. (1994a) Carcinogenicity of policosanol in Sprague Dawley rats: a 24 month study. Teratog Carcinog Mutagen 14, 239-249.

Aleman CL, Mas, R., Hernandez, C., Rodeiro, I., Cerejido, E., Noa, M., Capote, A., Menendez, R., Amor, A. \& Fraga, V. (1994b) A 12-month study of policosanol oral toxicity in Sprague Dawley rats. Toxicol Lett 70, 77-87.

Aneiros E, Mas R, Calderon B, Illnait J, Fernandez L, Castano G \& Fernandez JC (1995) Effect of policosanol in lowering cholesterol levels in patients with type II hypercholesterolemia. Curr Ther Res Exp. 56, 176-182.

Arruzazabala ML, Carbajal D, Mas R, Molina V, Castano G \& Gamez R (2003) Effects of D-003, a new compound purified from sugarcane wax, on platelet aggregation in healthy volunteers. Clin Drug Invest 23, 107-118.

Arruzazabala ML, Carbajal D, Mas R, Molina V, Valdes S \& Laguna A (1994) Cholesterol-lowering effects of policosanol in rabbits. Biol Res 27, 205-208.

Arruzazabala ML, Noa M, Menendez R, Mas R, Carbajal D, Valdes S \& Molina V (2000) Protective effect of policosanol on atherosclerotic lesions in rabbits with exogenous hypercholesterolemia. Braz J Med Biol Res 33, 835-840.

Aviram M (1993) Modified forms of low density lipoprotein and atherosclerosis. Atherosclerosis 98, 1-9.

Basu SK, Goldstein JL, Anderson GW \& Brown MS (1976) Degradation of cationized low density lipoprotein and regulation of cholesterol metabolism in homozygous familial hypercholesterolemia fibroblasts. Proc Natl Acad Sci U S A 73, 31783182.

Batista J, Stusser R, Saez F \& Perez B (1996) Effect of policosanol on hyperlipidemia and coronary heart disease in middle-aged patient. Int J Clin Pharm Res 34, 134137.

Bernard A \& Carlier H (1991) Absorption and intestinal catabolism of fatty acids in the rat: effect of chain length and unsaturation. Exp Physiol 76, 445-455.

Berthold HK, Unverdorben S, Degenhardt R, Bulitta M \& Gouni- Berthold I (2006) Effect of policosanol on lipid levels among patients with hypercholesterolemia or combined hyperlipidemia. A randomized controlled trial. JAMA 295, 2263-2269.

Bonanome A, Pagnan A, Biffanti S, Opportuno A, Sorgato F, Dorella M, Maiorino M \& Ursini F (1992) Effect of dietary monounsaturated and polyunsaturated fatty acids on the susceptibility of plasma low density lipoproteins to oxidative modification. Arterioscler Thromb 12, 529-533.

Bonné AC, den Bieman MG, Gillissen GF, van Lith HA \& van Zutphen LF (2002) Chromosomal localization of genes involved in biosynthesis, metabolism or transport of cholesterol in the rat. Cytogenet Genome Res 97, 183-186.

Brown DW, Haldman GA, Croft JB, Giles WH \& Mensah GH (2005) Racial or ethnic differences in hospitalization for heart failure among elderly adults: Medicare, 1990-2000. Am Heart J 150, 448-454. 
Cabrera L, Gonzalez V, Uribarri E, Sierra R, Laguna A, Magraner J, Mederos D \& Velazquez C (2002) Study of the stability of tablets containing $10 \mathrm{mg}$ of policosanol as active principle. Boll Chim Farm 141, 223-229.

Canetti M, Moreira M, Illnait J, Mas R, Fernandez L, Fernandez JC \& Castano G (1995a) One-year study of the effect of policosanol on lipid profile in patients with type II hypercholesterolemia. Adv Ther 12, 245-254.

Canetti M, Moreira M, Mas R, Illnait J, Fernandez L, Fernandez J, Diaz E \& Castano G (1995b) A two-year study on the efficacy and tolerability of policosanol in patients with type II hyperlipoproteinaemia. Int J Clin Pharmacol Res 15, 159165.

Canetti M, Moreira M, Mas R, Illnait J, Fernandez L \& Fernandez JC (1997) Effects of policosanol on primary hypercholesterolemia: A 3-year open-extension follow-up. Curr Ther Res Clin Exp 58, 868-875.

Carbajal D, Arruzazabala ML, Valdes S \& Mas R (1998) Interaction policosanolwarfarin on bleeding time and thrombosis in rats. Pharmacol Res 38, 89-91.

Carru C, Zinellu A, Galistu F, Barca M, Pasciu V, Lumbau F, Sanna B, Tadolini B \& Deiana L (2004) The evaluation of the oxidative state of native-LDL: three methods compared. J Biochem Biophys Methods 61, 271-281.

Castano G, Canetti M \& Moreira M (1995) Efficacy and tolerability of policosanol in elderly patients with type II hypercholesterolemia: A 12-month study. Curr Ther Res Clin Exp 56, 819-828.

Castano G, Fernandez L, Illnait J, Hernandez E, Fernandez J, Gamez R, Gutierrez C \& Alvarez E (2002a) A randomized, double-blind, placebo-controlled study of the efficacy and tolerability of policosanol in adolescents with type II hypercholesterolemia. . Curr Ther Res Clin Exp 63, 286-303.

Castano G, Fernandez L, Mas R, Illnait J, Fernandez J, Mesa M, Alvarez E \& Lezcay M (2002b) Comparison of the efficacy, safety and tolerability of original policosanol versus other mixtures of higher aliphatic primary alcohols in patients with type II hypercholesterolemia. Int J Clin Pharmacol Res 22, 55-66.

Castano G, Fernandez L, Mas R, Illnait J, Mesa M \& Fernandez J (2003a) Comparison of the effects of policosanol and atorvastatin on lipid profile and platelet aggregation in patients with dyslipidemia and type 2 diabetes mellitus. Clin Drug Invest 23, 639-650.

Castano G, Mas Ferreiro R, Fernandez L, Gamez R, Illnait J \& Fernandez C (2001a) A long-term study of policosanol in the treatment of intermittent claudication. Angiology 52, 115-125.

Castano G, Mas R, Arruzazabala ML, Noa M, Illnait J, Fernandez JC, Molina V \& Menendez A (1999) Effects of policosanol and pravastatin on lipid profile, platelet aggregation and endothelemia in older hypercholesterolemic patients. Int $J$ Clin Pharmacol Res 19, 105-116.

Castano G, Mas R, Fernandez J, Fernandez L, Alvarez E \& Lezcay M (2000a) Efficacy and tolerability of policosanol compared with lovastatin in patients with type II hypercholesterolemia and concomitant coronary risk factors. Curr Ther Res Clin $\operatorname{Exp}$ 61, 137-146. 
Castano G, Mas R, Fernandez L, Fernandez JC, Illnait J, Lopez LE \& Alvarez E (2000b) Effects of policosanol on postmenopausal women with type II hypercholesterolemia. Gynecol Endocrinol 14, 187-195.

Castano G, Mas R, Fernandez L, Illnait J, Gamez R \& Alvarez E (2001b) Effects of policosanol 20 versus $40 \mathrm{mg} /$ day in the treatment of patients with type II hypercholesterolemia: a 6-month double-blind study. Int J Clin Pharmacol Res 21, 43-57.

Castaño G, Más R, Fernández L, Illnait J, Hernández E, Fernández J, Gámez R, Gutiérrez C \& Alvarez E (2002) A randomized, double-blind, placebo-controlled study of the efficacy and tolerability of policosanol in adolescents with type II hypercholesterolemia. Current therapeutic research 63, 286-303.

Castano G, Mas R, Fernandez L, Illnait J, Mesa M, Alvarez E \& Lezcay M (2003b) Comparison of the efficacy and tolerability of policosanol with atorvastatin in elderly patients with type II hypercholesterolaemia. Drugs Aging 20, 153-163.

Castano G, Mas R, Gamez R, Fernandez J, Illnait J, Fernandez L, Mendoza S, Mesa M, Gutierrez JA \& Lopez E (2004) Concomitant use of policosanol and beta-blockers in older patients. Int J Clin Pharmacol Res 24, 65-77.

Castano G, Mas, R., Nodarse, M., Illnait, J. \& Fernandez, J.C. (1995) One-year of the efficacy and safety of policosanol (5 mg twice daily) in the treatment of type II hypercholesterolemia. Curr Ther Res Clin Exp 56, 296-303.

Castano G, Menendez R, Mas R, Amor A, Fernandez JL, Gonzalez RL, Lezcay M \& Alvarez E (2002c) Effects of policosanol and lovastatin on lipid profile and lipid peroxidation in patients with dyslipidemia associated with type 2 diabetes mellitus. Int J Clin Pharmacol Res 22, 89-99.

Cella LK, Van Cauter E \& Schoeller DA (1995) Diurnal rhythmicity of human cholesterol synthesis: normal pattern and adaptation to simulated "jet lag". Am J Physiol 269, E489-498.

Chen JT, Wesley R, Shamburek RD, Pucino F \& Csako G (2005) Meta-analysis of natural therapies for hyperlipidemia: plant sterols and stanols versus policosanol. Pharmacotherapy 25, 171-183.

Clark LT (2005) Issues in minority health: atherosclerosis and coronary heart disease in African Americans. Med Clin North Am 89, 977-1001.

Crespo N, Alvarez R, Mas R, Illnait J, Fernandez L \& Fernandez JC (1997) Effects of policosanol on patients with non-insulin-dependent diabetes mellitus and hypercholesterolemia: a pilot study Current therapeutic research 58, 44-51.

Crespo N, Illnait J, Mas R, Fernandez L, Fernandez J \& Castano G (1999) Comparative study of the efficacy and tolerability of policosanol and lovastatin in patients with hypercholesterolemia and noninsulin dependent diabetes mellitus. Int J Clin Pharmacol Res 19, 117-127.

Cubeddu LX, Cubeddu RJ, Heimowitz T, Restrepo B, Lamas GA \& Weinberg GB (2006) Comparative lipid-lowering effects of policosanol and atorvastatin: a randomized, parallel, double-blind, placebo-controlled trial. Am Heart J 152, 982 e981-985.

Davidson MH \& Robinson JG (2006) Lipid-lowering effects of statins: a comparative review. Expert Opin Pharmacother 7, 1701-1714. 
Davis PJ \& Poznansky MJ (1987) Modulation of 3-hydroxy-3-methylglutaryl-CoA reductase by changes in microsomal cholesterol content or phospholipid composition. Proc Natl Acad Sci U S A 84, 118-121.

Denke MA (1995) Lack of efficacy of low-dose sitostanol therapy as an adjunct to a cholesterol-lowering diet in men with moderate hypercholesterolemia. Am J Clin Nutr 61, 392-396.

Di Buono M, Hannah JS, Katzel LI \& Jones PJ (1999) Weight loss due to energy restriction suppresses cholesterol biosynthesis in overweight, mildly hypercholesterolemic men. J Nutr 129, 1545-1548.

Doggrell SA (2005) Berberine--a novel approach to cholesterol lowering. Expert Opin Investig Drugs 14, 683-685.

Dulin MF, Hatcher LF, Sasser HC \& Barringer TA (2006) Policosanol is ineffective in the treatment of hypercholesterolemia: a randomized controlled trial. Am J Clin Nutr 84, 1543-1548.

Ebine N, Jia X, Demonty I, Wang Y \& Jones PJ (2005) Effects of a water-soluble phytostanol ester on plasma cholesterol levels and red blood cell fragility in hamsters. Lipids 40, 175-180.

FDA (1998) Staking a claim to good health. http://www.fda.gov/fdac/features/1998/698 labl.html.

Fernandez J, Mas R, Castano G, Menendez R, Amor A, Gonzalez R \& Alvarez E (2001) Comparison of the efficacy, safety and tolerability of policosanol versus Fluvastatin in elderly hypercholesterolemic women. Clin Drug Invest 21, 103 113.

Field FJ, Born E \& Mathur SN (2004) Stanol esters decrease plasma cholesterol independently of intestinal ABC sterol transporters and Niemann-Pick C1-like 1 protein gene expression. J Lipid Res 45, 2252-2259.

Folch J, Lees M \& Sloane Stanley GH (1957) A simple method for the isolation and purification of total lipides from animal tissues. J Biol Chem 226, 497-509.

Gamez R, Aleman CL, Mas R, Noa M, Rodeiro I, Garcia H, Hernandez C, Menendez R \& Aguilar C (2001) A 6-Month Study on the Toxicity of High Doses of Policosanol Orally Administered to Sprague-Dawley Rats. J Med Food 4, 57-65.

Gamez R, Mendoza S, Mas R, Noa M, Arruzazabala L, Carbajal D, Castano G, Goicochea E, Mesa M \& Mendoza N (2003) Comparison of the cholesterollowering effects and toxicity of D-003 and

lovastatin in normocholesterolaemic rabbits. Drugs $R$ D 4, 219-229.

Gamez R, Mendoza, S., Mas, R., Noa, M., Arruzazabala, L., Carbajal, D., Castano, G., Goicochea, E., Mesa, M., Mendoza, N (2003) Comparison of the cholesterollowering effects and toxicity of D-003 and lovastatin in normocholesterolaemic rabbits. Drugs R D 4, 219-229.

Garcia-Pelayo MC, Garcia-Peregrin E \& Martinez-Cayuela M (2003) Modification of phospholipids fatty acid composition in reuber H35 hepatoma cells: effect on HMG-CoA reductase activity. $J$ Cell Biochem 90, 586-591.

Gouni-Berthold I \& Berthold HK (2002) Policosanol: clinical pharmacology and therapeutic significance of a new lipid-lowering agent. Am Heart J 143, 356-365. 
Greyling A, De Witt C, Oosthuizen W \& Jerling JC (2006) Effects of a policosanol supplement on serum lipid concentrations in hypercholesterolaemic and heterozygous familial hypercholesterolaemic subjects. Br J Nutr 95, 968-975.

Grundy SM \& Denke MA (1990) Dietary influences on serum lipids and lipoproteins. $J$ Lipid Res 31, 1149-1172.

Hardie DJ \& Carling D (1997) The AMP-activated protein kinase--fuel gauge of the mammalian cell? Eur J Biochem 246, 259-273.

Hargrove JL, Greenspan P \& Hartle DK (2004) Nutritional significance and metabolism of very long chain fatty alcohols and acids from dietary waxes. Exp Biol Med (Maywood) 229, 215-226.

Hobbs HH, Brown MS \& Goldstein JL (1992) Molecular genetics of the LDL receptor gene in familial hypercholesterolemia. Human Mutation 1, 445-466.

Hodge VJ, Gould SJ, Subramani S, Moser HW \& Krisans SK (1991) Normal cholesterol synthesis in human cells requires functional peroxisomes. Biophys Res Commun 181, 537-541.

Holvoet P, Mertens A, Verhamme P, Bogaerts K, Beyens G, Verhaeghe R, Collen D, Muls E \& Van de Werf F (2001) Circulating oxidized LDL is a useful marker for identifying patients with coronary artery disease. Arterioscler Thromb Vasc Biol 21, 844-848.

Holvoet P, Vanhaecke J, Janssens S, Van de Werf F \& Collen D (1998) Oxidized LDL and malonaldehyde-modified LDL in patients with acute coronary syndromes and stable coronary artery disease Circulation 98, 1487-1495.

Hulthe J \& Fagerberg B (2002) Circulating oxidized LDL is associated with subclinical atherosclerosis development and inflammatory cytokines (AIR Study). Arterioscler Thromb Vasc Biol 22, 1162-1167.

Hunninghake DB, Stein EA, Dujovne CA, Harris WS, Feldman EB, Miller VT, Tobert JA, Laskarzewski PM, Quiter E, Held J, Taylor AM, Hopper S, Leonard SB \& Brewer BK (1993) The efficacy of intensive dietary therapy alone or combined with lovastatin in outpatients with hypercholesterolemia. N Engl J Med 328, 1213-1219.

Jakobs BS \& Wanders RJ (1991) Conclusive evidence that very-long-chain fatty acids are oxidized exclusively in peroxisomes in human skin fibroblasts. Biochem Biophys Res Commun 178, 842-847.

Jolliffe N (1961) Dietary factors regulating serum cholesterol. Metabolism 10, 497-513. Jones PJ (1990) Use of deuterated water for measurement of short-term cholesterol synthesis in humans. Can J Physiol Pharmacol 68, 955-959.

Jones PJ (1999) Cholesterol-lowering action of plant sterols. Curr Atheroscler Rep 1, 230-235.

Jones PJ, MacDougall DE, Ntanios F \& Vanstone CA (1997) Dietary phytosterols as cholesterol-lowering agents in humans. Can J Physiol Pharmacol 75, 217-227.

Jones PJ, Pappu AS, Hatcher L, Li ZC, Illingworth DR \& Connor WE (1996) Dietary cholesterol feeding suppresses human cholesterol synthesis measured by deuterium incorporation and urinary mevalonic acid levels. Arterioscler Thromb Vasc Biol 16, 1222-1228. 
Jones PJ, Pencharz PB \& Clandinin MT (1985a) Absorption of 13C-labeled stearic, oleic, and linoleic acids in humans: application to breath tests. J Lab Clin Med 105, 647652.

Jones PJ, Pencharz PB \& Clandinin MT (1985b) Absorption of 13C-labeled stearic, oleic, and linoleic acids in humans: application to breath tests. J Lab Clin Med 105, 647652.

Jones PJ, Scanu AM \& Schoeller DA (1988) Plasma cholesterol synthesis using deuterated water in humans: effect of short-term food restriction. J Lab Clin Med 111, 627-633.

Jonkers IJ, Ledeboer M, Steens J, Smelt AH \& Masclee AA (2000) Effects of very long chain versus long chain triglycerides on gastrointestinal motility and hormone release in humans. Dig Dis Sci 45, 1719-1726.

Junker LH \& Davis RA (1989) Receptor-mediated uptake of low density lipoprotein stimulates bile acid synthesis by cultured rat hepatocytes. J Lipid Res 30, 19331941.

Kabir Y \& Kimura S (1993) Biodistribution and metabolism of orally administered octacosanol in rats. Ann Nutr Metab 37, 33-38.

Kabir Y \& Kimura S (1995) Tissue distribution of (8-14C)-octacosanol in liver and muscle of rats after serial administration. . Ann Nutr Metab 39, 279-284.

Kassis AN \& Jones PJ (2006) Lack of cholesterol-lowering efficacy of Cuban sugar cane policosanols in hypercholesterolemic persons. Am J Clin Nutr 84, 1003-1008.

Kassis AN, Marinangeli CP, Jain D, Ebine N \& Jones PJ (2006) Lack of effect of sugar cane policosanol on plasma cholesterol in golden syrian hamsters. Atherosclerosis Epub ahead of print.

Kobayashi M, Ishida F, Takahashi T, Taguchi K, Watanabe K, Ohmura I \& Kamei T (1989) Preventive effect of MK-733 (simvastatin), an inhibitor of HMG-CoA reductase, on hypercholesterolemia and atherosclerosis induced by cholesterol feeding in rabbits. Jpn J Pharmacol 49, 125-133.

Kopprash S, J. P, Kuhlisch E, Fuecker K, Temelkova-Kurktschiev T, Hnefeld M, Kuhne H, Julius U \& Graessler J (2002) In vivo evidence for increased oxidation of circulating LDL in impaired glucose tolerance. Diabetes 31, 3102-3112.

Kosoglou T, Statkevich P, Johnson-Levonas AO, Paolini JF, Bergman AJ \& Alton KB (2005) Ezetimibe: a review of its metabolism, pharmacokinetics and drug interactions. Clin Pharmacokinet 44, 467-494.

Kris-Etherton PM \& Dietschy J (1997) Design criteria for studies examining individual fatty acid effects on cardiovascular disease risk factors: human and animal studies. Am J Clin Nutr 65, 1590S-1596S.

Lapenna D, Ciofani G, Pierdomenico SD, Giamberardino MA \& Cuccurullo F (2001) Reaction conditions affecting the relationship between thiobarbituric acid reactivity and lipid peroxides in human plasma. Free Radic Biol Med 31, 331335.

Law MR (2000) Plant sterol and stanol margarines and health. West J Med 173, 43-47.

Lee M, Lu K \& Patel SB (2001) Genetic basis of sitosterolemia. Curr. Opin. Lipidol. 12, 141-149.

Lee T (1979) Characterization of fatty alcohol:NAD+ oxidoreductase from rat liver. J Biol Chem J Biol Chem 254, 2892-2896. 
Lefkowitz RJ \& Willerson JT (2001) Prospects for cardiovascular research. Jama 285, 581-587.

Leitch CA \& Jones PJ (1993) Measurement of human lipogenesis using deuterium incorporation. J Lipid Res 34, 157-163.

Lin Y, Rudrum M, van der Wielen RP, Trautwein EA, McNeill G, Sierksma A \& Meijer GW (2004) Wheat germ policosanol failed to lower plasma cholesterol in subjects with normal to mildly elevated cholesterol concentrations. Metabolism 53, 13091314.

Mandel H, Getsis M, Rosenblat M, Berant M \& Aviram M (1995) Reduced cellular cholesterol content in peroxisome-deficient fibroblasts is associated with impaired uptake of the patient's low density lipoprotein and with reduced cholesterol synthesis. J Lipid Res 36, 1385-1391.

Mannaerts GP, Van Veldhoven PP \& Casteels M (2000) Peroxisomal lipid degradation via beta- and alpha-oxidation in mammals. Cell Biochem Biophys 32 Spring, 7387.

Marinangeli CP, Kassis AN, Jain D, Ebine N, Cunnane SC \& Jones PJ (2007) Comparison of composition and absorption of sugarcane policosanols. Br J Nutr 97, 381-388.

Mas R (2000) Monographs- Policosanol: Hypolipidemic, antioxidant, treatment of atherosclerosis. Drugs of the future 25, 569-586.

Mas R, Castano G, Fernandez J, Gamez RR, Illnait J, Fernandez L, Lopez E, Mesa M, Alvarez. E \& Mendoza S (2004) Long- term effects of policosanol on older patients with Type 2 diabetes. Asia Pacific Journal of Clinical Nutrition 13, 101S.

Mas R, Castano G, Fernandez L, Illnait J, Fernandez J \& Alvarez E (2001) Effects of policosanol on lipid profile and cardiac events in older hypercholesterolemic patients with coronary disease. Clinical drug investigation 21, 485-497.

Mas R, Castano G, Illnait J, Fernandez L, Fernandez J, Aleman C, Pontigas V \& Lescay M (1999) Effects of policosanol in patients with type II hypercholesterolemia and additional coronary risk factors. Clin Pharmacol Ther 65, 439-447.

McLaughlin J, Grazia Luca M, Jones MN, D'Amato M, Dockray GJ \& Thompson DG (1999) Fatty acid chain length determines cholecystokinin secretion and effect on human gastric motility. Gastroenterology 116, 46-53.

Mendoza s, Gamez, R., Noa, M., Mas, R., Castano, G., Mesa, A. R., Mesa, M. \& de Armas, M. (2001) Comparison of the effects of D-003 and policosanol on lipid profile and endothelial cells in normocholesterolemic rabbits. Curr Ther Res Clin $\operatorname{Exp}$ 62, 209-220.

Menendez R, Amor AM, Gonzalez RM, Fernandez JC, Rodeiro I, Zayas M \& Jimenez S (2000a) Effects of policosanol treatment on the susceptibility of low density lipoprotein (LDL) isolated from healthy volunteers to oxidative modification in vitro. J Clin Pharmacol 50, 255-262.

Menendez R, Amor AM, Gonzalez RM, Fraga V \& Mas R (1996) Effect of policosanol on the hepatic cholesterol biosynthesis of normocholesterolemic rats. Biol Res 29, 253-257.

Menendez R, Amor AM, Gonzalez RM, Fraga V \& Mas R (1999a) Effect of policosanol on the hepatic cholesterol biosynthesis of normocholesterolemic rats. Biol Res 29, 253-257. 
Menendez R, Amor AM, Gonzalez RM, Jimenez S \& Mas R (2000b) Inhibition of rat microsomal lipid peroxidation by the oral administration of D002. Braz J Med Biol Res 33, 85-90.

Menendez R, Amor AM, Rodeiro I, Gonzalez RM, Gonzalez PC, Alfonso JL \& Mas R (2001a) Policosanol modulates HMG-CoA reductase activity in cultured fibroblasts. Arch Med Res 32, 8-12.

Menendez R, Arruzazabala L, Mas R, Del Rio A, Amor AM, Gonzalez RM, Carbajal D, Fraga V, Molina V \& Illnait J (1997) Cholesterol-lowering effect of policosanol on rabbits with hypercholesterolaemia induced by a wheat starch-casein diet. $\mathrm{Br} J$ Nutr 77, 923-932.

Menendez R, Fernandez SI, Del Rio A, Gonzalez RM, Fraga V, Amor AM \& Mas RM (1994) Policosanol inhibits cholesterol biosynthesis and enhances low density lipoprotein processing in cultured human fibroblasts. Biol Res 27, 199-203.

Menendez R, Fraga V, Amor AM, Gonzalez RM \& Mas R (1999b) Oral administration of policosanol inhibits in vitro copper ion-induced rat lipoprotein peroxidation. Physiol Behav 67, 1-7.

Menendez R, Marrero D, Mas R, Fernandez I, Gonzalez L \& Gonzalez RM (2005) In vitro and in vivo study of octacosanol metabolism. Arch Med Res 36, 113-119.

Menendez R, Mas R, Amor AM, Gonzalez RM, Fernandez JC, Rodeiro I, Zayas M \& Jimenez S (2000c) Effects of policosanol treatment on the susceptibility of low density lipoprotein (LDL) isolated from healthy volunteers to oxidative modification in vitro. Br J Clin Pharmacol 50, 255-262.

Menendez R, Mas R, Amor AM, Rodeiros I, Gonzalez RM \& Alfonso JL (2001b) Inhibition of cholesterol biosynthesis in cultured fibroblasts by D003, a mixture of very long chain saturated fatty acids. Pharmacol Res 44, 299-304.

Mensah GH, Mokdad AH, Ford ES, Greenlund KJ \& Croft JB (2005) State of disparities in cardiovascular health in the United States. Circulation 111, 1233-1241.

Mesa AR, Mas R, Noa M, Hernandez C, Rodeiro I, Gamez R, Garcia M, Capote A \& Aleman CL (1994) Toxicity of policosanol in beagle dogs: one-year study. Toxicol Lett 73, 81-90.

Mirkin A, Mas R, Martinto M, Boccanera R, Robertis A, Poudes R, Fuster A, Lastreto E, Yanez M, Irico G, McCook B \& Farre A (2001) Efficacy and tolerability of policosanol in hypercholesterolemic postmenopausal women. Int J Clin Pharmacol Res 21, 31-41.

Molina C, Arruzazabala ML, Carbajal D, Mas R \& Valdes S (1998) Effect of policosanol on arterial blood pressure in rats. Study of pharmacological interaction with nifedipine and propanolol. Arch Med Res 29, 21-24.

Monograph (2004) Policosanol. Altern Med Rev 9, 312-317.

Murphy KJ, Saint DA \& Howe PR (2004) Lack of effect of sugar cane and sunflower seed policosanols on plasma cholesterol in rabbits. Asia Pac J Clin Nutr 13, S69.

Murray CJ \& Lopez AD (1997a) Global mortality, disability, and the contribution of risk factors: Global Burden of Disease Study. Lancet 349, 1436-1442.

Murray CJ \& Lopez AD (1997b) Mortality by cause for eight regions of the world: Global Burden of Disease Study. Lancet 349, 1269-1276. 
Ng CH, Leung KY, Huang Y \& Chen ZY (2005) Policosanol has no antioxidant activity in human low-density lipoprotein but increases excretion of bile acids in hamsters. J Agric Food Chem 53, 6289-6293.

Noa M, Mas R, de la Rosa MC \& Magraner J (1995) Effect of policosanol on lipofundininduced atherosclerotic lesions in rats. J Pharm Pharmacol 47, 289-291.

Noa M, Mas R \& Mesa R (1997) Effect of policosanol on circulating endothelial cells in experimental models in Sprague-Dawley rats and in rabbits. J Pharm Pharmacol 49, 999-1002.

Noa M, Mendoza S, Mas R \& Mendoza N (2003) Effect of policosanol on carbon tetrachloride-induced acute liver damage in Sprague-Dawley rats. Drugs RD 4, 29-35.

Olivier LM \& Krisans SK (2000) Peroxisomal protein targeting and identification of peroxisomal targeting signals in cholesterol biosynthetic enzymes. Biochim Biophys Acta 1529, 69-102.

Ordovas JM (2006) Genetic interactions with diet influence the risk of cardiovascular disease. Am J Clin Nutr 83, S443-446.

Ortensi G, Gladstein J, Valli H \& Testone P (1997) A comparative study of policosanol versus simvastatin in elderly patients with hypercholesterolemia. Curr Ther Res Clin Exp 58, 390-401.

Otto J, Ordovas JM, Smith D, van Dongen D, Nicolosi RJ \& Schaefer EJ (1995) Lovastatin inhibits diet induced atherosclerosis in F1B golden Syrian hamsters. Atherosclerosis 114, 19-28.

Pallauda C, Gueguenb R, Sassa C, Growc M, Chengc S, Siesta G \& Visvikisa S (2001) Genetic influences on lipid metabolism trait variability within the Stanislas Cohort Journal of Lipid Research 42, 11879-11890.

Parker TS, McNamara DJ, Brown C, Garrigan O, Kolb R, Batwin H \& Ahrens EH, Jr. (1982) Mevalonic acid in human plasma: relationship of concentration and circadian rhythm to cholesterol synthesis rates in man. Proc Natl Acad Sci US A 79, 3037-3041.

Parthasarathy S, Khoo JC, Miller E, Barnett J, Witztum JL \& Steinberg D (1990) Low density lipoprotein rich in oleic acid is protected against oxidative modification: implications for dietary prevention of atherosclerosis. Proc Natl Acad Sci US A 87, 3894-3898.

Patents US (1994) Mixture of higher primary aliphatic alcohols, its obtention from sugar cane wax and its pharmaceutical uses. Patent \# 5856316 Biol Res 27, 199-203.

Pearson TA, Blair SN, Daniels SR, Eckel RH, Fair JM, Fortmann SP, Franklin BA, Goldstein LB, Greeland P, Grundy SM, Hong Y, Miller NH, Lauer RM, Ockene IS, Sacco RL, Sallis JF, Smith SC, Stone NJ \& Taubert KA (2002) AHA Guidelines for Primary Prevention of Cardiovascular Disease and Stroke: 2002 Update Circulation 106, 388-391.

Perman J, Fagerlund C \& Hulthe J (2004) Methodological aspects of measuring oxidized low density lipoproteins in human serum and plasma. Scand J Clin Lab Invest 64, 753-755.

Pons P, Illnait J, Mas R, Rodriguez M, Aleman C, Fernandez JC, Fernandez L \& Martin M (1997) A comparative study of policosanol versus probucol in patients with hypercholesterolemia. Curr Ther Res Clin Exp 58, 26-35. 
Pons P, Mas R, Illnait J, Fernández L, Rodríguez M, Robaina C \& Fernández JC (1992) Efficacy and safety of policosanol in patients with primary hypercholesterolemia. Curr Ther Res Clin Exp 52, 507-513.

Pons P, Rodríguez M, Más R, Illnait J, Fernández L, Robaina C \& Fernández JC (1994a) One-year efficacy and safety of policosanol in patients with type II hypercholesterolemia. Curr Ther Res Clin Exp 55, 1084-1092.

Pons P, Rodriguez M, Robaina C, Illnait J, Mas R, Fernandez L \& Fernandez JC (1994b) Effects of successive dose increases of policosanol on the lipid profile of patients with type II hypercholesterolaemia and tolerability to treatment. Int J Clin Pharmacol Res 14, 27-33.

Raeini-Sarjaz M, Vanstone CA, Papamandjaris AA, Wykes LJ \& Jones PJ (2001) Comparison of the effect of dietary fat restriction with that of energy restriction on human lipid metabolism. Am J Clin Nutr 73, 262-267.

Reeves PG (1997) Components of the AIN-93 diets as improvements in the AIN-76A diet. $J$ Nutr 127, 838S-841S.

Reiner Z, Tedeschi-Reiner E \& Romic Z (2005) Effects of Rice Policosanol on Serum Lipoproteins, Homocysteine, Fibrinogen and C-Reactive Protein in Hypercholesterolaemic Patients. Clin Drug Invest 5, 701-707.

Rizzo WB, Craft DA, Dammann AL \& Phillips MW (1987) Fatty alcohol metabolism in cultured human fibroblasts. Evidence for a fatty alcohol cycle. J Biol Chem 262, 17412-17419.

Rodriguez-Echenique C, Mesa R, Mas R, Noa M, Menendez R, Gonzalez RM, Amor AM, Fraga V, Sotolongo V \& Laguna A (1994) Effects of policosanol chronically administered in male monkeys (Macaca arctoides). Food Chem Toxicol 32, 565575.

Rodriguez MD \& Garcia H (1994) Teratogenic and reproductive studies of policosanol in the rat and rabbit. Teratog Carcinog Mutagen 14, 107-113.

Rodriguez MD \& Garcia H (1998) Evaluation of peri- and post-natal toxicity of Policosanol in rats. Teratog Carcinog Mutagen 18, 1-7.

Rodriguez MD, Sanchez M \& Garcia H (1997) Multigeneration reproduction study of policosanol in rats. Toxicol Lett 90, 97-106.

Sallee VL (1979) Permeation of long-chain fatty acids and alcohols in rat intestine. Am J Physiol 236, E721-727.

Sallee VL \& Dietschy JM (1973) Determinants of intestinal mucosal uptake of short- and medium-chain fatty acids and alcohols. $J$ Lipid Res 14, 475-484.

Salter AM \& White DA (1996) Effects of dietary fat on cholesterol metabolism: regulation of plasma LDL concentration. Nutrition Research Reviews 9, 241-257.

Santosa S, Demonty I, Lichtenstein AH \& Jones PJ (2007) Cholesterol metabolism and body composition in women: the effects of moderate weight loss. Int $J$ Obes (Lond) 31, 933-941.

Schachter M (2005) Chemical, pharmacokinetic and pharmacodynamic properties of statins: an update. Fundam Clin Pharmacol 19, 117-125.

Scott RPW \& Perry JA (1998) Introduction to analytical gas chromatography, 2nd ed. Marcel Dekker, New York. 
Sigurdardottir V, Fagerberg B \& Hulthe J (2002) Circulating oxidized low-density lipoprotein (LDL) is associated with risk factors of the metabolic syndrome and LDL size in clinically healthy 58-year-old men (AIR study). J Intern Med 252, 440-447.

Simonen P, Gylling H, Howard AN \& Miettinen TA (2000) Introducing a new component of the metabolic syndrome: low cholesterol absorption. Am J Clin Nutr 72, 82-88.

Simonen P, Gylling H \& Miettinen TA (2002) Acute effects of weight reduction on cholesterol metabolism in obese type 2 diabetes. Clin Chim Acta 316, 55-61.

Singh DK, Li L \& Porter TD (2006) Policosanol inhibits cholesterol synthesis in hepatoma cells by activation of AMP- kinase. Pharmacol Exp Ther 318, 10201026.

Spady DK \& Dietschy JM (1988) Interaction of dietary cholesterol and triglycerides in the regulation of hepatic low density lipoprotein transport in the hamster. J Clin Invest 81, 300-309.

Stancu C \& Sima A (2001) Statins: mechanism of action and effects. J Cell Mol Med 5, 378-387.

Tedeschi-Reiner E, Reiner Z, Romic Z \& Ivankovic D (2005) A randomized, doubleblind, placebo-controlled study of the antilipemic efficacy and tolerability of food supplement policosanol in patients with moderate hypercholesterolemia. Lijec Vjesn 127, 273-279.

Thompson DF \& Montarella KE (1993) Understanding financial conflicts of interest. $N$ Engl J Med Volume 329, 573-576.

Uribarri E, Laguna A, Sierra R \& Ricardo Y (2002) Physico-mechanical characterization of policosanol, a novel hypocholesterolemic drug. Drug Dev Ind Pharm 28, 8993.

Vanstone CA, Raeini-Sarjaz M, Parsons WE \& Jones PJ (2002) Unesterified plant sterols and stanols lower LDL-cholesterol concentrations equivalently in hypercholesterolemic persons. Am J Clin Nutr 76, 1272-1278.

Varady KA, Wang Y \& Jones PJ (2003) Role of policosanols in the prevention and treatment of cardiovascular disease. Nutr Rev 61, 376-383.

Vistisen B, Mu H \& Hoy CE (2006) The recovery of 13C-labeled oleic acid in rat lymph after administration of long chain triacylglycerols or specific structured triacylglycerols. Eur J Nutr 45, 363-368.

Wanders RJ, van Grunsven EG \& Jansen GA (2000) Lipid metabolism in peroxisomes: enzymology, functions and dysfunctions of the fatty acid alpha- and betaoxidation systems in humans. Biochem Soc Trans 28, 141-149.

Wanders RJ, Vreken P, Ferdinandusse S, Jansen GA, Waterham HR, van Roermund CW \& Van Grunsven EG (2001) Peroxisomal fatty acid alpha- and beta-oxidation in humans: enzymology, peroxisomal metabolite transporters and peroxisomal diseases. Biochem Soc Trans 29, 250-267.

Wang MF, Lian HZ, Mao L, Zhou JP, Gong HJ, Qian BY, Fang Y \& Li J (2007) Comparison of Various Extraction Methods for Policosanol from Rice Bran Wax and Establishment of Chromatographic Fingerprint of Policosanol. J Agric Food Chem 55, 5552-5558. 
Wang Y, Ebine N, Jia X, Jones PJ, Fairow C \& Jaeger R (2005) Very long chain fatty acids (policosanols) and phytosterols affect plasma lipid levels and cholesterol biosynthesis in hamsters. Metabolism 54, 508-514.

Wang Y, Vanstone CA, Parsons WD \& Jones PJH (2004) Validation of a single-isotopelabelled cholesterol tracer approach for measuring human cholesterol absorption Lipids 39, 87-91.

Wang YW, Jones PJ, Pischel I \& Fairow C (2003) Effects of policosanols and phytosterols on lipid levels and cholesterol biosynthesis in hamsters. Lipids $\mathbf{3 8}$, 165-170.

Whitcomb RW, Linehan WM \& Knazek RA (1988) Effects of long-chain, saturated fatty acids on membrane microviscosity and adrenocorticotropin responsiveness of human adrenocortical cells in vitro. $J$ Clin Invest 81, 185-188.

Whitney EN \& Rolfes SR (2002) Digestion, absorption, and transport. In Understanding Nutrition, pp. 61-91. Belmont, CA: Wadsworth.

Williams KJ \& Tabas I (1995) The response-to-retention hypothesis of early atherogenesis. Arterioscler Thromb Vasc Biol 15, 551-561. 


\section{APPENDICES}


Appendix 1.1: Animal study ethics approval

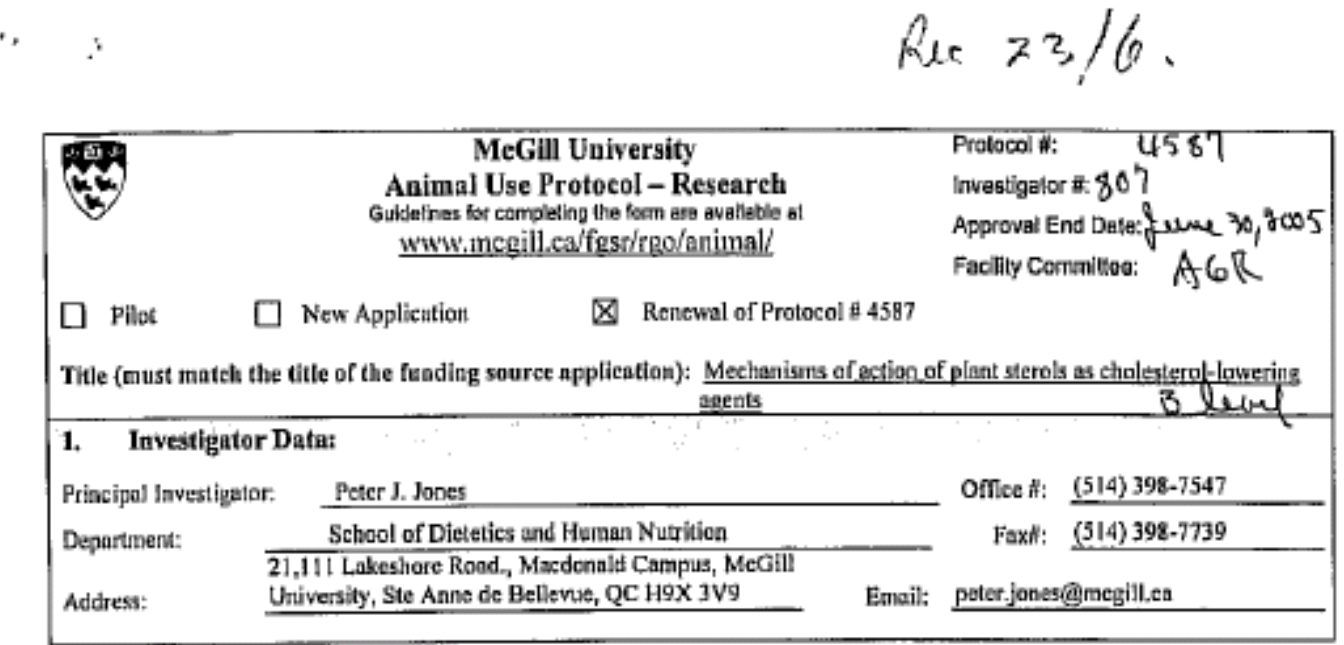

\begin{tabular}{|c|c|c|c|c|c|}
\hline Name: & Peter Jones & Work $\mathrm{H}$ : & $398-7547$ & Emergency 䒪: & $457-5122$ \\
\hline Narne: & Naoyaki Eblae & Work A: & $398-8660$ & Eanergency \#̈: & $630-2499$ \\
\hline
\end{tabular}

\begin{tabular}{|c|c|}
\hline 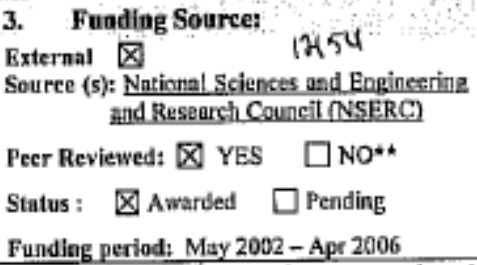 & $\begin{array}{l}\text { Internal } \square \\
\text { Source (s): } \\
\text { Peer Revlewed: } \square \text { YES } \square \text { NO** } \\
\text { Status: } \square \text { Awarded } \square \text { Pending } \\
\text { Funding period: }\end{array}$ \\
\hline \multicolumn{2}{|c|}{ 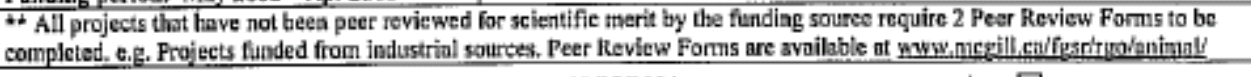 } \\
\hline Propesed Start Date of Animal Use (d/m/y): & $19 / 07 / 2004 \quad$ or ongoing $\square$ \\
\hline & $24 / 09 / 2004$ \\
\hline
\end{tabular}

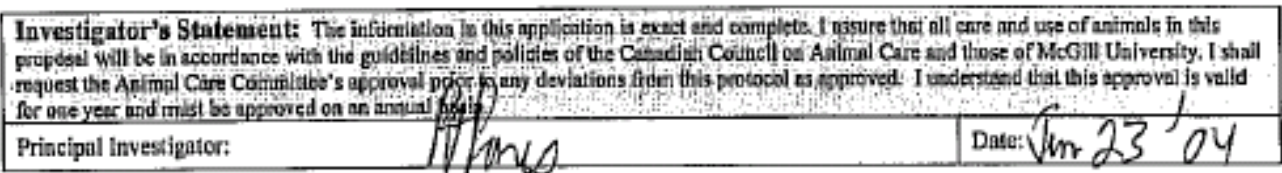

\begin{tabular}{l} 
Approval Signatures: \\
\hline Chair, Facility Animal Care Committee:
\end{tabular}


Appendix 1.1: Animal study ethics approval

\begin{tabular}{|c|c|c|c|}
\hline $\begin{array}{l}\text { Researeh } \\
\text { study (ine } \\
\text { and under } \\
\text { certifies th } \\
\text { which qua } \\
\text { indieate th }\end{array}$ & $\begin{array}{l}\text { Sonnel and Qualificn } \\
\text { the Principal Investiga } \\
\text { ate/graduate student, an } \\
\text { personuel listed here ha } \\
\text { them to perform the pro } \\
\text { e has rend this protocol. }\end{array}$ & $\begin{array}{l}\text { Ions: List the names of all individuals who w } \\
\text { r) and their employment classification (invest } \\
\text { fellow). Indicate any training received (e.g w } \\
\text { e suitable training and/or experience, or will b } \\
\text { edures described in the protocol. Each person } \\
\text { (Space will expand as needed.) }\end{array}$ & $\begin{array}{l}\text { with animals in this } \\
\text { in, researth assistant } \\
\text { res, etc.). The PI } \\
\text { the specific iraining } \\
\text { ation must sign to }\end{array}$ \\
\hline Name & Clnssifiention & Training Informution & Signnture \\
\hline $\begin{array}{l}\text { Peter JH Jones } \\
\text { Naoyuki Ebine }\end{array}$ & $\begin{array}{l}\text { Principal investigator } \\
\text { Postdoctoral fellow }\end{array}$ & $\begin{array}{l}\text { Animal handling workshop and course at } \\
\text { University of'Toronto } \\
\text { Animal handling workshop at McGill (2002) }\end{array}$ & \\
\hline
\end{tabular}

\section{Summary (In language that will be uaderstood by members of the general public)}

a) Rationnle: Describe, in a short paragraph, the overall aim of the situdy and its potential benefit to human/animal beatth or to the advancement of scientific knowledge.

Plant sterols, comprise of a class of compounds similar in chemical structure to cholesterol, interfere with the normal process of intestinal cholesterol absorption. In bumen, the decline in intestinal cholesterol absorption celn produce a decrease in circulating LDL-cholesterol of $10-15 \%$, commensurate with a reduction in risk of cardiovaseular disease by up to $25 \%$. Despite widespread use of plant sterols, the exact mechanisms through which these compounds interfere with cholesterol uptake remains to be precisely identified. The overall objective of the present program of research will be to uncover the one or more sites of action at which plant sterols restrict intestinal cholesterol absorption, and to examine how dietary factors impact on each of these sites. In order to examine these hypotheses, a combination of whole body animal, organ and cell model systems will be used. In vivo systems will utilize the hamster which has been shown to serve as an appropriate model for studying human lipid metabolism. Tagged cholesterol and plant stetols will be used to examine comparative rates of uptake, and cholesterol exclusion, of major sterols sitosterol and campesterol using these different model systems. Results of this study should provide data which twill result in a clearer understanding of the precise point if action of plant sterols, thus assist in explaining the relative efficacy of plant sterols under different dietary conditions. Such information will provide significant new understanding of plant sterol metabolism which should lead to the development of dietary guidelines consistent with maximization off health benefits obtained through use of dietary plant sterols.

b) Specific Objectives of the Study: Summarize in point form the primary objectives of this study.

1. Evalunte the action of specific plant sterols/stanots and their esters on cholesterol absorption at the level of duodenal deesterificntion.

2. Compare effects of diet plant sterols/stanoIs and their esters on (i) circulating phytosterol/stanol levels, (ii) fat soluble and provitamin levels, (iii) red cell fragility and (iv) indices of cell proliferatioss risk.

c) Progress Report: If this is a renewal of an ongoing project, brienly summarize what was accomplished during the prior approval period and indicate if and how the current goals differ from those in the original application.

The data obtained form our previous study shows that plant stanol ester has potent cholesterol lowering effect compure to free plant sterols/stanols or esterified sterols. In this study, we will focts on two different stanol esters to find an nppropriate way to fortify the efficacy of plant stanols in cholesterol-lowering by esterification. Moreover, it has been claimed that lons chain fatty alcohol (policosanol) intake influences the lipid profiles, cholesterol levels and thus benefits human cardiovascular system, raising the question as to whether a synergy effect exisis between lorg fatty alcohols and stanol esters in lowering cholesterol levels. Addressing these questions in an unimal model will permit assessment of the efficacy of fatty alcohols and plant stanol esters alone as well as combination on cholesterol levels in plasmn and de novo cholesterol biosynthesis. This will also permit exploring the mechanisms by which these agents lower cliolesterol levels.

d) Summary of Procedures for Animal Use Report to the CCAC : Using key words only, list the procedures used (e.g. anaesthesia, breeding colony, injection IP, gavnge, drug administration, major survival surgery, euthanasia by exsanguination, behaviournl studies). Refer to Appendix 1of the Guidelines for a more complete list of strggested key words. Specinl diet, stupplementation of plant sterols/stanols and their esters, deuterium and ${ }^{13} \mathrm{C}$ administration, anaesthesia, euthantsin with excess inhalation of $\mathrm{CO}_{2}$, blood collection, tissue collection 
Appendix 1.1: Animal study ethics approval

\section{Animals To Be Used}

a) Purpose of Animal Use (Check one):

1. \. Studies of a fundamental nature/basic retearch

2. Studies for medical purposes relating to human/animal diseases/disorders

3. $\square$ Regulatory testing

4. D. Development of products/appliances for human'veterinary medicine

b) Will the project involve breeding animals? NO $\triangle$ YES $\square$

Will the project involve the generation of genetically altered animals? NO $\otimes \quad$ YES $\square$ Will field studies be conducted? NO $₫$ YES $\square$

\begin{tabular}{|c|c|c|c|c|c|c|}
\hline & Species 1 & Species 2 & Species 3 & Species 4 & Speciers 5 & Species 6 \\
\hline Species & Elamster & & & & & \\
\hline Supplier/Source & Charles River & & & & & \\
\hline Strain & Golden Syrian & & & & & \\
\hline Sex & Male & & & & & \\
\hline Age/Tht & 4 weeks $/ 100 \mathrm{~g}$ & & & & & \\
\hline \# To be purchased & 96 & & & & & \\
\hline $\begin{array}{l}\text { \# Produced by in- } \\
\text { louse breeding }\end{array}$ & 0 & & & & & \\
\hline $\begin{array}{l}\text { \# Other } \\
\text { (c.g. field studies) }\end{array}$ & 0 & & & & & \\
\hline $\begin{array}{l}\text { \#nceded at one } \\
\text { time }\end{array}$ & 96 & & & & & \\
\hline \# per cage & 1 & & & & & \\
\hline TOTAL\# /YEAR & 96 & & & & & \\
\hline
\end{tabular}

\section{Justification of Animal Usage}

a) Please justify the number of animals requested for cach species described above, bascd on the experimental objectives of the project. Include information on experimental and control troups, \# per troup, and failure rates. Also justify in lerms of statistienl requirements, product yield, etc. For breeding, specify how many adulis are used, number of offspring produced, and how many offspring are used in experimental procedures. Use the table below when applicable (space will expand as needed).

The number of animals required (12 per group) is the minimum required to achieve adequate statistical power among the eight dietary treatment groups. It is necessary to include these eight treatment groups in order to examine the action of various forms of plant stanol csters on cholesterol absorption at the level of duodenal de-esterification and their effects on (i) circulating

phytosterol/stanol levels, (ii) fat soluble and provitamin levels, (iii) red cell fragility and (iv) indices of cell proliteration risk.

Despite all recent studies, mechanisms of action of plant sterol/stanol mixtures on plasma lipids remain inadequately

characterized. Better definition of the precise site of effect of different typically consumed plant sterol forms is required to

determine the mechanisms of action. 
Appendix 1.1: Animal study ethics approval

9. Standinrd Operinting Procedures (SOPs)

Complete this section if you plan to use any of the UACC SOPs listed below. It is UACC policy that these SOPs be used when applicable. Any proposed variation of the SOPs tmust be described and justified. The Standard Operating Procedures can be found at the UACC website at www mcgill,ca/fgst/rgo/animall. The completed end signed SOP form must be attached to the protocol.

Check all SOPs that will be used:

Blood Collection (UACCH1)

冈 Eroduction of Monoclonal Antibodies (UACC\#7)

Annesthesia (rodents) (UACC $\sharp 2$ )

$\square$ Production of Polyclonal Antibodies(UACCHB )

Analgesin (rodents/larger species) (UACCH3)

Breeding (transgenics/knockouts) (UACC $\$ 4$ )

$\square \quad$ Collection of Amphibian Oooytes (UACCH9)

Transgenio Generation (UACCH5)

Knockout/in Generation (UACCH6)

\section{Description of Procedures}

a) For each experimental group, describe all procedures and techniques in the order in which they will be performed surgical procedures, immunizations, behavioural tests, immobilization and restraint, food/wwater deprivation, requirements for post-operative care, sample collection, substance administration, special monitoring, etc. If a procedure is covered by an SOP, write "as per SOP", no further detail is required. Appendix 2 of the Guidelines provides a sample list of points tha should be addressed in this section.

All animals will bo fed their respective test diets daily. Stanol and stanol esters will be mixed into oil as previously described (Vanstone $\mathrm{CA}$, et al. J Nutr Biochem 2002). Body weight will be measured once a week. Food consumption will be mensured once every three days by weighing the difference of food cups before and atter the feeding.

On day 29 of the feeding period, the dietary cholesterol will be labeled with ${ }^{13} \mathrm{C}$ tracer. Al 24 hours after the addition of the label, animals will be weighted. After that animal will be euthatized by dectapitation with nnesthesin in $\mathrm{CO}_{2}$ clanther, followed by blood and tissue somples (liver, duodenum, jejunum and ileum) will be collected.

b) Field Studies - Provide all relevant details. Procedures to be conducted (e.g. surgery, blood collection, thgging etc.) should be described above.

\section{Not Applicable}

Method of capture/restraint, duration of captivity, potential injury/mortality, monitoring frequency:

Transporation and /or housing of animals in the field:

Sptcial handling required:

Capture of non-tnrget species, potentinl injury/mortality:

Will captured animuls be relensed at or near the capture site YES $\square$ NO $\square$

If not, specify if they will be relocated to other locations and/or populations.

Describe any potentíal ecological disruption this study may cause;

It is the responsibility of the investigator to obtain all necessary permits for wark with wild life. Copies af these permits must be forwarded to the Research Ethics Officer (Animal Studies) when they are obtained. 
Appendix 1.1: Animal study ethics approval

\begin{tabular}{|c|c|c|c|c|c|}
\hline Test Agents or Procedures & $\begin{array}{l}\text { \# of Animnls } \\
\text { and Species } \\
\text { Per Group }\end{array}$ & $\begin{array}{l}\text { Dosage and/or Route } \\
\text { of Administration }\end{array}$ & \# of endpoints & $\begin{array}{l}\text { Other variables (i.e. scx } \\
\text { weight, genotypes, etc.) }\end{array}$ & $\begin{array}{l}\text { Total number of } \\
\text { anImals }\end{array}$ \\
\hline c.g. 2 Drugs & e.g. 6 mils & $\begin{array}{l}\text { e.g. } .03, .05 \mathrm{mg} / \mathrm{kg} \\
\text { - IM, IP } \\
\text { (4 variables) }\end{array}$ & $\begin{array}{l}\text { e.g. } 1,7,10 \\
\text { days (3. } \\
\text { variables) }\end{array}$ & $\begin{array}{l}\text { c.g. Male, Female } \\
\text { groups (2 vuriubles) }\end{array}$ & $\begin{array}{l}\text { e.g. } 2 \times 6 \times 4 \times 3 \text {, } \\
2=288\end{array}$ \\
\hline $\begin{array}{l}\text { No cholesterol diet } \\
\text { Atherogenic diet (AD) with } 0.1 \% \\
\text { cholesterol }\end{array}$ & $\begin{array}{l}12 \\
12\end{array}$ & $\begin{array}{l}- \\
-\end{array}$ & $\begin{array}{l}30 \text { dnys } \\
30 \text { days }\end{array}$ & $\begin{array}{l}\text { Mule } \\
\text { Mnle }\end{array}$ & $12 \times 8=96$ \\
\hline $\begin{array}{l}A D \text { with free stunal } \\
A D \text { with stanol ascorbnte ester }\end{array}$ & $\begin{array}{l}12 \\
12\end{array}$ & $\begin{array}{l}1 \mathrm{~g} / \mathrm{kgBW} \\
1 \mathrm{gkgBW}\end{array}$ & $\begin{array}{l}30 \text { duys } \\
30 \text { dnys }\end{array}$ & $\begin{array}{l}\text { Mule } \\
\text { Mnle }\end{array}$ & \\
\hline$A D$ with ferulayl stanot & 12 & $10 \mathrm{kgBW}$ & 30 diuss & & \\
\hline$A D$ with policosanol & 12 & $1 \mathrm{gkgBW}$ & 30 diys & Male & \\
\hline $\begin{array}{l}\text { AD with policosanol plus stanot } \\
\text { ascorbate ester }\end{array}$ & 12 & $1 \mathrm{~g} / \mathrm{kgBW}$ & 30 days & Male & \\
\hline $\begin{array}{l}\text { AD with policosanal plus f́cruloyl } \\
\text { stano! }\end{array}$ & 12 & $1 \mathrm{~g} / \mathrm{kgBW}$ & 30 days & Male & \\
\hline
\end{tabular}

b) Please justify the need for live animals versus alternate methods (c.g, tissue culture, computer simulation).

In order to evaluate the action of specific plant stanol esters ot cholesterol absorption at the level of duodenal de-esterification and compare the effects of these materials on (i) circulating phytosterol/stanol levels, (ii) fat soluble and provitamin levels, (iii) red cell fragility and (iv) indices of cell proliferation, live animals are required.

Due to the complex nature of animal physiology it is imperntive that we study the intact animal model rather than alternative method such as tissue culture or computer simulation.

e) Describe the charucteristics of the animal species selected that justifies its use in the proposed study (consider characteristics such as body size, species, strain, data from previous studies or unique anatomic/physiological features)

The hamster has been identified as a good model of human lipid metabolism. The hamster is representative of humans in terms of liver and small intestine physiology. Past work in our laboratory, in both rabbits and hamsters, has demonstruted that plant sterols work in a manner dependent on their degree of saturation, to signifieantly suppress cholesterol absorption, while desuppressing cholesterol biosynthesis. Previous data from hamster experiments suggests that additional mechanisms are at work, indicating that plant sterol/stanol action occurs over and above simple effects at the level of absorption. Declines in circulating cholesterol levels were observed in hamsters provided with very small doses of plant sterols by subcutaneous injection, quantities that would be expected to be absorbed from feeding levels capable of cholesterol lowering (Vanstone CA, et al. J Nutr Biochem 2002).

8. Animal Husbandry and Care

a) Specinl cages No 1 YES $\$ Specify: Individual metabolic cages for colfection of spilled food

Special diet $\quad$ NO $\square$ YES $\otimes$ Specify: Treatment materials are provided through diet

Special hnndling NO $\otimes \quad$ YES $\square$ Specify:

b) Is there any component to the proposed procedures which will result in immunosuppresslon or decrensed immune function (c.g. stress, radintion, steroids, cliemotherapeutics, and genetic modification of the immune system)?

No $\square$ YES $\square$ Specify:

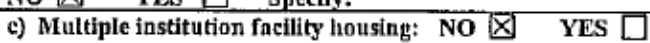
Indicate all facilities where animals will be housed:

Indicnte area(s) where animal use procedures will be conducted:

\begin{tabular}{llll|} 
Building: & $\begin{array}{l}\text { Institute of } \\
\text { Parnsitology } \\
\text { Institute of } \\
\text { Building: }\end{array}$ & Room No: & TBA \\
& Room No: & TBA
\end{tabular}

If animal housing and animal use are in different locations, briefly describe procedures for transporting animals: Not applicable 
Appendix 1.1: Animal study ethics approval

\begin{tabular}{|c|c|c|c|}
\hline \multicolumn{4}{|c|}{ 9. Standard Opernting Procedures (SOPsis) } \\
\hline \multicolumn{4}{|c|}{$\begin{array}{l}\text { Complete this section if you plan to use any of the UACC SOPs listed below. It is UACC policy that these SOPs be used when } \\
\text { applicable. Any proposed variation of the SOPs tmust be described and justified. The Standard Operating Procedures can be found } \\
\text { at the UACC website at www womcgill,ca/fger/rgo/animal. The completed end signed SOP form must be attached to the protocol. }\end{array}$} \\
\hline \multicolumn{4}{|l|}{ Check all SOPs that will be used: } \\
\hline Blood Collection (UACCH1) & 区 & Eroduction of Monoclonal Antibodies (UACC \#7) & $\square$ \\
\hline Annesthesia (rodents) (UACC \#2) & $\square$ & Production of Polyclonal Antibodies(UACCH8) & $\square$ \\
\hline Analgesin (rodents/larger species) (UACCH3) & $\square$ & Collection of Amphibian Oooytes (UACCHA ) & $\square$ \\
\hline Breeding (transgenics/knockouts) (UACCH4) & $\square$ & Rodent Surgery (UACCH10) & $\square$ \\
\hline Transgenic Generation (UACCHS) & $\square$ & $\begin{array}{l}\text { Neonatal Rodent Angesthesin and Euthanasin } \\
\text { (UACCH11) }\end{array}$ & $\square$ \\
\hline Knockout'in Generation (UACCH6) & $\square$ & & $\square$ \\
\hline
\end{tabular}

\section{Description of Procedures}

a) For each experimentnl group, describe all procedures and techniques in the order in which they will be performed surgical procedures, immunizations, behavioural tesis, immobilization and restraint, food/wwater deprivation, requirements for post-operative care, sample collection, substance administration, special monitoring, etc. If a procedure is covered by an SOP, write "as per SOP", no farther detail is required. Appendix 2 of the Guidelines provides a sample list of points that should be addressed in this section.

All animals will bo fed their respective test diets daily. Stanol and stanol esters will be mixed into oil as previously deseribed (Vanstone CA, et al. J Nutr Biochem 2002). Body weight will be measured once a week. Food consumption will be mensured once every three days by weighing the difference of food cups before and after the feeding.

On day 29 of the feeding period, the dietary cholesterol will be lubeled with ${ }^{13} \mathrm{C}$ tracer. Al 24 hours after the addition of the label, animals will be weighted. After that animal will be euthatized by decapitation with nnesthesin in $\mathrm{CO}_{2}$ chamber, followed by blood and tissue samples (liver, duodenum, jejunum and ileum) will be collected.

b) Field Studies - Provide all relevant details. Procedures to be conducted (e.g. surgery, blood collection, tagging etc.) should be described above.

\section{Not Applicable}

Method of capture/restraint, duration of captivity, potential injury/mortality, monitoring frequency:

Transporation and /or housing of animals in the field:

Specit handling required:

Capture of non-tnrget species, potentin! injury/mortalify:

Will captured animuls be relensed at or near the capture site YES $\square$ NO

If not, specify if they will be relocated to other locations and/or populations.

Describe any potentíal ecological disruption this study may cause:

It is the responsibility of the investigator to obtain all necessary permits for work with wildife. Copies af these permits must be forwarded to the Resenrch Ethics Officer (Animal Studies) when they are obtained. 
Appendix 1.1: Animal study ethics approval

c) Pre-Annesthetic/Anaesthetic/Analgesic Agents: List all drugs that will be used to ninimize pain, distress or discomfort Table will expand as needed.

\begin{tabular}{|l|l|l|l|l|l|}
\hline Species & Agent & Dosage (mig/kg) & $\begin{array}{l}\text { Total volume(ml) per } \\
\text { administration }\end{array}$ & Route & Frequency \\
\hline & & & & & \\
\hline
\end{tabular}

d) Adninistration of non-anesthetic substances: List all non-anaesthetic agents under study in the experimental component of the protocol, including but not limited to drugs, infectious agents, viruses (trble will expand as needed).

\begin{tabular}{|c|c|c|c|c|c|}
\hline Species & Agent & Dosage (mg/kg) & $\begin{array}{l}\text { Total volume (ml) per } \\
\text { administrution }\end{array}$ & Route & Frequency \\
\hline Hnmster & $\begin{array}{c}\text { 13 C labeled- } \\
\text { cholesterol }\end{array}$ & $1 \mathrm{~g} / \mathrm{kg} \mathrm{BW}$ & $0.1 \mathrm{ml}$ & Oral & Once at day 29 \\
\hline
\end{tabular}

e) Endpoints: 1) Experimental - for cach experimental group indicate survival time.

2) Clinical - describe the conditions, complications, and criteria (e.g. $>20 \%$ wt.loss, tumour size, vocalizing, lack of grooming) that would lead to euthanasia of an animal before the expected completion of the experiment (specify per species and project if multiple projects involved).

The study will continue for a total duration of 30 days. If at any time an animal develops diarrhen, abscesses, respiratory infection, skin infections and other diseases signs they will be annesthetized followed by euthanusia. If any animal experiences a weight loss of greuter than $20 \%$ of their original weight of fails to consume $25 \%$ of their nomal diet, they will be euthanized

Specify person(s) who will be responsible for animal monitoring and post-operative care

Nnme: Naoyuki Ebine

Phoneif: 398-8660

f) Method of Euthanasin - According to CCAC guidelines, justification must be provided for use of any physical method of euthanasia swithout prior use of anaesthesia (justify here):

Decapitation with unesthesiu

Specify Species

\begin{tabular}{|c|c|}
\hline & $\square$ anesthetic overdose, list agenU/doseroute: \\
\hline & $\square$ exsanguirtation with anaesthesia, list agent/dose'route: \\
\hline Hatnster & 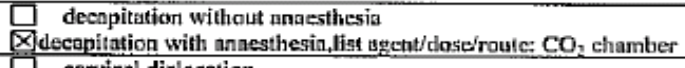 \\
\hline & $\square$ cervical dislocation \\
\hline & $\square \mathrm{CO}_{2}$ ehumber \\
\hline & D other (specify) \\
\hline & [ not upplicable (explain) \\
\hline
\end{tabular}

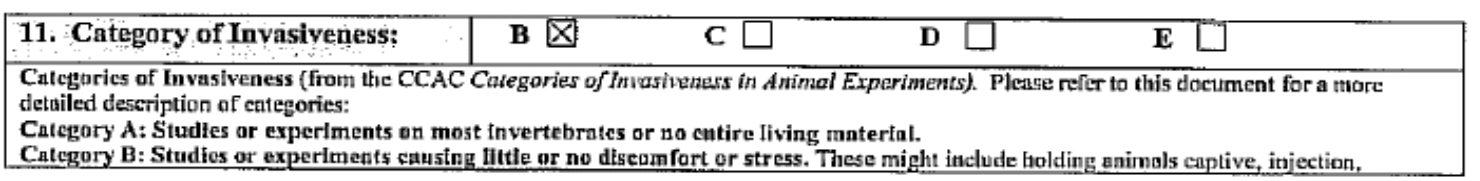


Appendix 1.1: Animal study ethics approval

page 7

percutaneous blood sampling, accepted euthannsis for tissue haryest, acute non-survival experiments in which the animals are completely ansesthetized.

Cntegory C: Studtes or experiments involving mfnor stress or pain of short durntion. These might include cannulation or catheterizations of blood vessels or body cuvities under anaesthesia, minor surgery under annesthesia, stuch as biopsy; shon perjods of restruint, overnight food and/or water deprivation which exceed periods of abstinence in nature; behavioural experiments an conscious animals that involve short-term stressful restruint.

Cntegory D: Studles or experiments that Involve moderate to severe distress ar discomfort. These might include major sutgery under anaesthesiu with subsequent recovery, prolonged (severnl hours or more) periods of physjcal restraini; inductjon of behevioural stresses, immunization with complete Freund's adjuvant, appliention of noxious stimuli, procedures that produce pain, production of transgenies (in accordance with University policy).

Category E: Procedures that involve infiettng severe paln, near, at or above the pain threshald of unnuncstlictizet, conscious animals. Not confined to but may include exposure to noxious stimuli or agents whose effects are unknown; exposure to drugs or ehemicals at levels that (mey) murkedly impair physiological systems and which catuse death, severe paitn or extreme distress or pilysical traume on unannesthetized animals. Aceording to Unfiersity policy, E level studies are not permitted.

12. Potentinl Hazards to Personnel and Animals It is the responsibility of the investigntor to obtain the necessary Biohazard and/or Radiation Safety permits before this protocol is subtnitted for roview. A copy of these certificates must be attached, if applicable.

No hazardous materials will be used in this study: $区$

a) Indicate which of the following will be used in animals:

GToxic chemicals $\square$ Rndioisotopes $\square$ Carcinogens $\square$ Infectious agents $\square$ Transplantable tumours

b) Complete the following table for each agent to be used (use additional page as required).

\begin{tabular}{|l|l|l|l|}
\hline Agent & & & \\
\hline Dosnge & & & \\
\hline Route of admfnistration & & & \\
\hline Frequency of administration & & & \\
\hline Duration of administration & & & \\
\hline Number of animals lavolved & & & \\
\hline Survival time after administration & & & \\
\hline
\end{tabular}

c) After administration the animuls will be housed in:

the animal care facility

$\bigotimes$ labortory under supervision of laboratory personnel

Plense note that cages must be approprintely labeled at all times.

d) Describe potential health risk (5) to humans or animals:

e) Describe measures that will be used to reduce risk to the environment and all project and animal facility personnel:

13. Reviewer's Modifientions (to be completed by ACC only): The Animal Care Committee has mide the following modification(s) to this protocol during the review process. Plense make these changes to your copy. You must comply with the recommended changes as a condition of approval. 


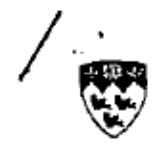

\section{McGill University Animal Care Committee AMENDMENT to Animal Use Protocol whyw.megill.enirgo/cnimat}

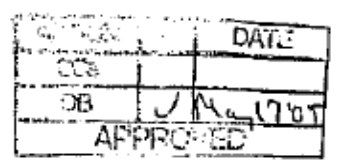

Principal Investigator:

Protocol Title:

Peter Jones Mechanism of action of plant slerols as chalestero-lowering

Unit, Dept. \& Address: agents - ACM my ceníce of Cxclute 21111 Lakeshore Road., Macdonald Campus, McGil University, Ste Anne de Bellevus, QC HOX 3V

Emall: peler.jones@mcgill.ce

Lẹvel:

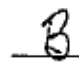

Protocol \# 4587

Phone: (514) 398-7547

Fax:

(514) $398-7739$

National Sclences and Engineering and Research Council (NSERC); The Funding: Advanced Food and Materials Network

1. ADDITIONAL ANIMALS REQUESTED: (vistify addifional anlmal mumbers in bax 5 below)

\begin{tabular}{|c|c|c|c|c|c|c|c|c|c|c|}
\hline Specles & Strain & $\begin{array}{c}\text { Suppller } \\
\text { Source }\end{array}$ & $\begin{array}{c}\text { \# Animals } \\
\text { Purchased }\end{array}$ & $\begin{array}{c}\text { \#By } \\
\text { Breeding }\end{array}$ & Age & Sex & Weight & $\begin{array}{c}\text { WNeeded at } \\
\text { One Time }\end{array}$ & $\begin{array}{c}\text { \#Cage } \\
\text { Yotal Per } \\
\text { Year }\end{array}$ \\
\hline & & & & & & & & & & \\
\hline & & & & & & & & & & \\
\hline
\end{tabular}

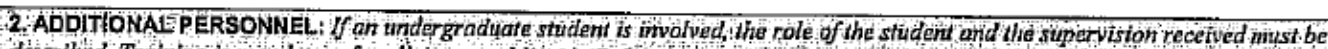

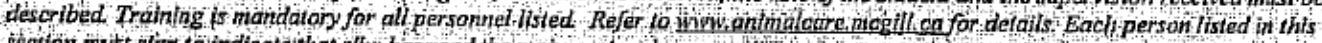
section must sign to indicate that she has read the main prolocol

\begin{tabular}{|c|c|c|c|c|}
\hline Name & Classification & Animal Related Training & $\begin{array}{l}\text { Occupational Health } \\
\text { \& Safety Program }\end{array}$ & Signature \\
\hline Christopher Marlnangeli & Graduate Student & $\begin{array}{l}\text { Animal Handling Workshop at } \\
\text { McGill (2004) }\end{array}$ & & geh \\
\hline Deepak Jain & Graduate Student & $\begin{array}{l}\text { Anlmal Handling Workshop at } \\
\text { McG!li (2004) }\end{array}$ & . & Deriai \\
\hline Amira Kassis & Graduate Student & $\begin{array}{l}\text { Animal Handling Workshop at } \\
\text { MeGill (2004) }\end{array}$ & & \\
\hline
\end{tabular}

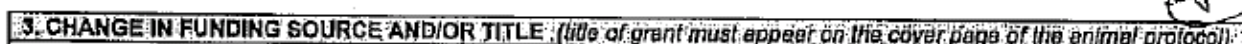
National Sclences and Engineering and Research Council (NSERC); The Advanced Food and Materlals Network

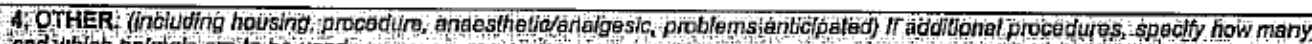
and which enintols are to be used. Added Objectives:

1. Evaluate the action of policosanols on cholesterol absorption at the level of duodenal de-esterificalion.

2. Compare effects of dietary policosanol on (i) clrculating and tissue policosanol levels, (il) circulating cholesterol levels, (iii) red call fragility. (iv) endogenous cholesterol synthesls.

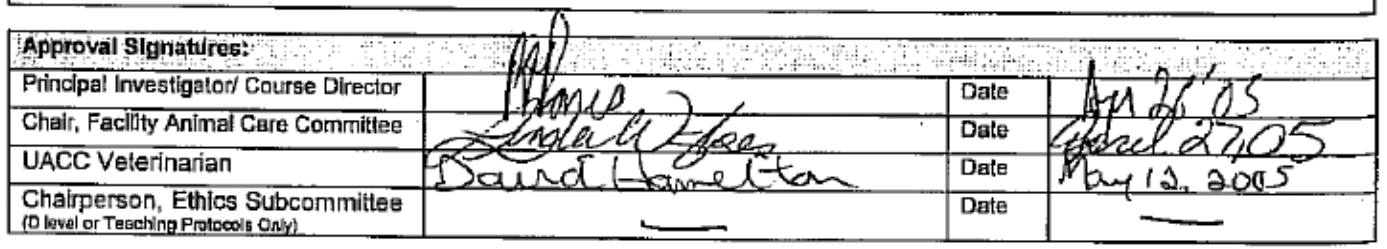


Appendix 1.2: Human study ethics approval

\section{Tricour Pdice}

\section{McGill}

Faculty of Medicine 3655 Promenade Sir William Osler Montreal, QC H3G IY6

October 26, 2004

Dr. Peter J. H. Jones

Professor of Nutrition

School of Dietetics and Human Nutrition

21,111 Lakeshore

Ste-Anne-De-Bellevue, Quebec H9X 3V9
Faculté de médecine

3655, Promenade Sir William Osler

Montréal, QC, H3G 1 Y6
Fax/Télécopieur: (514) 398-3595

Dear Dr. Jones,

We have received correspondence in support of the research proposal AØØ-M58-04A entitled "Evaluation of Very Long Chain Fatty Acids/Alcohols and Plant Sterols as functional Food Ingredients for Cholesterol-Lowering in Hypercholesterolemic Humans", whch was re-submitted for review by the Institutional Review Board, Faculty of Medicine at its meeting of September 13, 2004.

The responses and revisions were found to be acceptable and we are pleased to inform you that final approval for study (August 2, 2004), revised consent form (October 15, 2004), and English and French recruitment advertisements (October 15, 2004) was provided on October 26, 2004, valid until September 2005. The certificate of approval (executed) is enclosed.

A review of all research involving human subjects is required on an annual basis in accord with the date of initial review and approval. Should any modification to the study or unanticipated development occur prior to the next review, please advise the IRB promptly.

Yours sincerely,

$$
\text { D. Celme ficura }
$$

Celeste Johnston, DEd, RN

Co-Chair,

Institutional Review Board

cc: A09-M58-04A 
Appendix 1.2: Human study ethics approval

\section{McGill \\ Faculty of Medicine \\ 3655 Promenade Sir William Osler \\ Montreal, QC H3G IY6 \\ Faculté de médecine \\ 3655, Promenade Sir William Osler \\ Montréal, QC, H3G 1 Y \\ Fax/Télécopieur: (514) 398-3595}

\section{Certification Of ETHICAL ACCEPTABILITY FOR RESEARCh} INVOLVING HUMAN SUBJECTS

The Faculty of Medicine Institutional Review Board consisting of:

$\begin{array}{ll}\text { CELESTE JOHNSTON, DED } & \text { ARTHUR CANDIB, MED } \\ \text { CATHERINE GARDNER, BSC } & \text { RICHARD HAMILTON, MD } \\ \text { LAWRENCE HUTCHISON, MD } & \text { NELl MACDONALD, MD } \\ \text { WILSON MILLER, MD, PHD } & \text { ROBERT L. MUNRO, BCL } \\ \text { ROBERTA PALMOUR, PHD } & \text { MARGARET SWAINE, BA }\end{array}$

has examined the research project A09-M58-04A entitled "Evaluation of Very Long Chain Fatty Acids/Alcohols and Plant Sterols as functional Food Ingredients for Cholesterol-Lowering in Hypercholesterolemic Humans"

as proposed by:

Peter Jones

to

Applicant

Granting Agency, if any

and consider the experimental procedures to be acceptable on ethical grounds for research involving human subjects.

October 26, 2004

Date

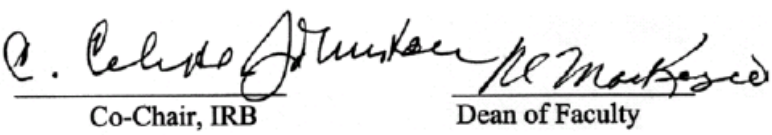

Institutional Review Board Assurance Number: FWA 00004545 
November 5, 2004

\section{SUBJECT CONSENT FORM OF RESEARCH PROTOCOL}

Evaluation of Very Long Chain Fatty Acids/Alcohols as Functional Food Ingredients for Cholesterol-Lowering in Hypercholesterolemic individuals.

Patient Name:

Protocol Number:

School of Dietetics \& Human Nutrition, Macdonald Campus, McGill University

Principal Investigator: Dr. Peter Jones

Phone:

(514) 398-7547, 398-7527

Contact Physician: Dr. Eric Lillie

Phone:

(514) 457-9355

The nature of the procedure or treatment, its risks and/or benefits, and possible alternatives, are as follows:

\section{NATURE AND DURATION OF PROCEDURE}

The aim of this study is to examine the cholesterol-lowering effectiveness of fat based alcohols. The fat based alcohols are found in wheat, rice and sugar cane waxes. They have been previously shown to lower cholesterol in people with elevated blood lipids. The above mentioned aim will be studied by assessing the effect of dietary consumption of $10 \mathrm{mg} /$ day of sugar cane wax derived alcohols for 28 days with regard to (i) lipid and fat-soluble vitamin levels in the blood, (ii) cholesterol absorption and (iii) cholesterol synthesis rate, in individuals with high blood cholesterol levels.

As part of a pre-screening visit, a fasting blood sample of $20 \mathrm{ml}$ (four teaspoons) will be taken for the laboratory to confirm the absence of health abnormalities and to measure your cholesterol level. Following qualification for the study, you will be invited 
back for further screening where a fasting blood sample of $20 \mathrm{ml}$ will be obtained to obtain a complete blood count. An electrocardiogram may be performed at the discretion of the physician in charge. Prior to the start of the study, you will be examined by a physician to make sure that you are in good health.

You will also need to ensure that you (1) have not consumed any lipid lowering drugs, including fish oils or cholesterol lowering medication within the past 6 months, (2) do not have any history of chronic use of insulin, systemic antibiotics, corticosteroids, androgens or phenytoin, (3) have not developed thyroid disease, diabetes mellitus or kidney, heart or liver disease within the past 3 months or cancer (evidence of any active lesion, chemotherapy or surgery within the past year), (4) do not chronically use fiber or stimulant laxatives (greater than 2 doses/wk), (5) do not have a history of eating disorders or binging, and (6) do not perform exercises greater than 15 miles/wk or $3000 \mathrm{kcal} / \mathrm{wk}$.

The total length of the study will be 2 phases of 28 days each, separated by a washout period of 28 days. You will be asked to maintain your habitual diet and level of physical activity. During the 2 phases, you will be required to visit the Mary Emily Clinical Nutrition Research Center located at 7 Maple Avenue, Ste-Anne-de-Bellevue, to consume a snack provided by the unit kitchen. You will be asked to come in daily between 5 and $7 \mathrm{pm}$. You will need to limit coffee intake to one cup per day or less and abstain from alcohol use over the course of the study.

A $15 \mathrm{ml}$ blood sample will be taken on day 1 of each phase, in order to assess blood cholesterol levels. Also, 4 days before the end of each of the four intervention phases, you will be orally given a small amount of cholesterol labeled with ${ }^{13} \mathrm{C}$, a nonradioactive tag. This will be provided as a single small mass within a warmed pat of margarine. In order to track this non-radioactive tag, fasting blood samples $(15 \mathrm{ml})$ will then be obtained at $0,24,48,72$ and $96 \mathrm{hr}$ after consumption of the tagged cholesterol, in order to establish the amount of cholesterol being absorbed inside your body. All blood samples will subsequently be analysed for different blood lipid components. 
Additionally, on day 28 of each phase, you will also be requested to consume three tablespoons of water tagged with deuterium (a non-radioactive tag, also commonly called heavy water) in order to establish the amount of cholesterol being produced inside your body. Heavy water is almost identical to regular water $\left(\mathrm{H}_{2} 0\right)$, except that a heavier form of hydrogen called deuterium replaces the hydrogen part of the water molecule. This tag is non-radioactive and poses no toxicity hazard.

Each venipuncture will take approximately 5 minutes. The total amount of blood drawn during each phase of the study will be $105 \mathrm{ml}$. The total blood volume required for this trial will be $210 \mathrm{ml}$, plus $40 \mathrm{ml}$ for screening to total $250 \mathrm{ml}$.

In order to monitor your caloric intake and your level of activity, you will be asked to fill a questionnaire once a week during the 2 phases.

\section{POTENTIAL RISKS AND/OR BENEFITS}

There are no known reports of adverse effects with plant based alcohols, which will be used in this study. Volunteers may run the risk of becoming anemic, but this will be tested for at the end of each diet phase, and anemic volunteers will receive iron supplementation (ferrous gluconate, $325 \mathrm{mg}$ orally, once a day, for two weeks). In the event that you feel any discomfort during the experimental trial, a physician, Dr. Lillie, will be available to contact at any time. Dr. Lillie can be reached at (514) 4579355. If any health problems are identified in the clinical tests conducted during this experiment, you will be informed of such by the doctor associated with the study.

The content of the procedures have been fully explained to me, and all experimental aspects have been identified. I have had the opportunity to ask questions concerning any and all aspects of the project and procedures involved, and may continue in the future to ask further questions at any time, as it is my right to do so. I am aware that I may refuse to participate as well as withdraw my consent at any time. I acknowledge that no guarantee or assurance has been given by anyone as to the results to be 
obtained and that my participation in this study is completely voluntary.

Confidentiality of records concerning my involvement in this project will be maintained in an appropriate manner. Samples will not be utilized for any additional analyses, nor stored for any prolonged period, nor shared with any other group, other than is indicated in the protocol, without my specific consent.

$\mathrm{I}$, , the undersigned, hereby consent to participate as a subject in the above named research project conducted by McGill University. I have read the above description with one of the investigators, . I have been made aware of all the procedures, advantages and disadvantages of the study, which have been explained to me. I acknowledge that, in compensation for the inconvenience of the study schedule, I will receive $\$ 500$. If I decide to withdraw before completion or should the study be terminated early, I will receive an appropriate pro-rated fraction of this amount. I will also receive access to my test results when they become available.

I acknowledge receiving a copy of this consent form and all appropriate attachments and agree to be contacted by a member of the Research Ethics Committee.

Investigator Signature of Subject

Witness

Date

Time 
October 15,2004

\section{NUTRITION STUDY McGill University MACDONALD CAMPUS}

The School of Dietetics and Human Nutrition at McGill University is conducting a study to investigate the effects of plant-based cholesterol lowering agents on blood lipid levels.

The study is open to individuals who meet the following criteria:

- Male or post-menopausal women, overweight, aged 40 to 80 years old

- Non smoker

- In good health, not taking any medication for a chronic condition including cholesterol lowering therapy

The total length of the study will be 4 phases of 28 days each, where all meals will be provided by the Mary Emily Clinical Nutrition Research Unit in Ste-Anne-de-Bellevue. You will be required to come in to consume at least one meal a day at the Mary Emily Clipic.

\begin{tabular}{|c|c|}
\hline 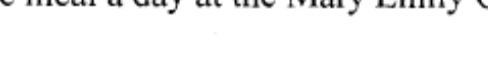 & $\begin{array}{l}\text { DATE OF I.R.B. } \\
\text { APPROVAL }\end{array}$ \\
\hline COMPENSATION $\$ 1,000$ & ОСТ 262004 \\
\hline If Interested, Please Call: & $\begin{array}{l}\text { Faculty of Medicine } \\
\text { McGill University }\end{array}$ \\
\hline
\end{tabular}

(514) 398-7624

Amira Kassis, Study Coordinator

Dr. Peter Jones, Professor 
October 15,2004

\section{ETUDE EN NUTRITION \\ Université McGill CAMPUS MACDONALD}

L'École de Diététique et Nutrition Humaine de l'Université McGill

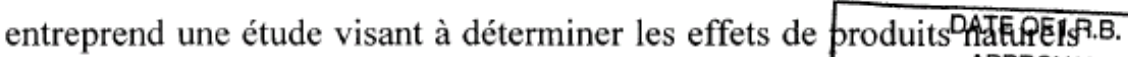
d'origine végétale sur le cholesterol sanguin.

OCT 262004

L'étude est offerte aux individus répondant aux critères suivivales

- Être un homme ou une femme post-menopauseé présenttant unt University surpoids, agé(e) entre 40 et 80 ans

- Être non fumeur

- Être en bonne santé, ne pas prendre de médicament pour une maladie chronique, ni de médicament réduisant le taux de cholestérol.

L'étude consistera en quatre phases de 28 jours, où tous les repas seront fournis par l'Unité de Recherche en Nutrition Clinique Mary Emily située à Ste-Anne-de-Bellevue. Au moins un des repas devra être consommé à la clinique Mary Emily.

\section{COMPENSATION $\$ 1,000$}

Si vous êtes interessé(e), veuillez d'appeler au:

(514) 398-7624

Amira Kassis, Coordinatrice d'étude

Dr. Peter Jones, Professeur 


\section{Policosanol study 2004-2005 screening information form}

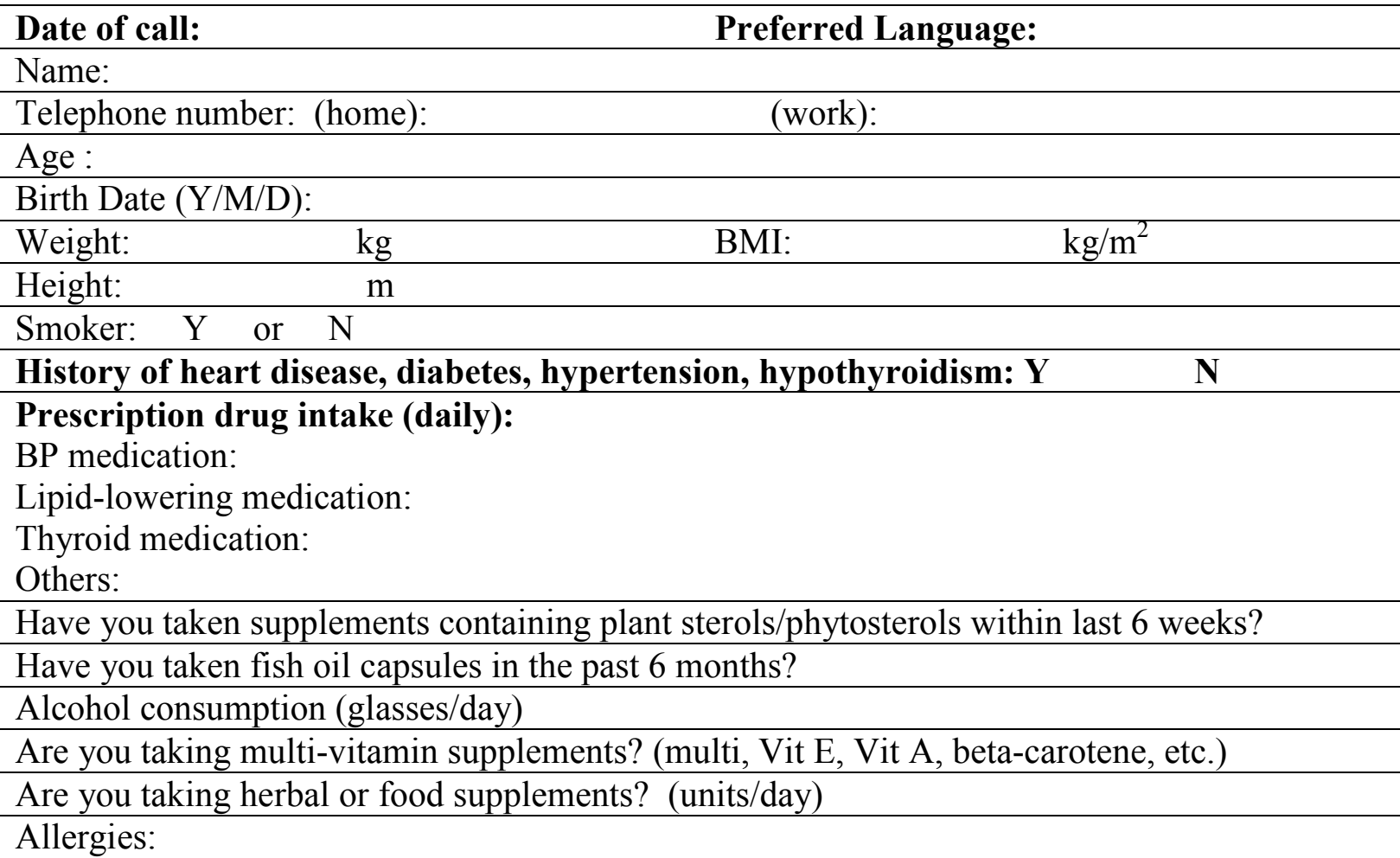

\begin{tabular}{|l|l|}
\hline \multicolumn{1}{|c|}{ Appointment } \\
\hline \hline \multicolumn{1}{|c|}{ Blood Test (Fasting) } & \\
\hline Date: & Date: \\
Time: & Time: \\
\hline Date: & Date: \\
Time: & Time: \\
\hline Eligibility for study: \\
\hline Start date of study: \\
\hline Subject Code / Identification Number: \\
\hline
\end{tabular}


Policosanol study 2004-2005 screening information form

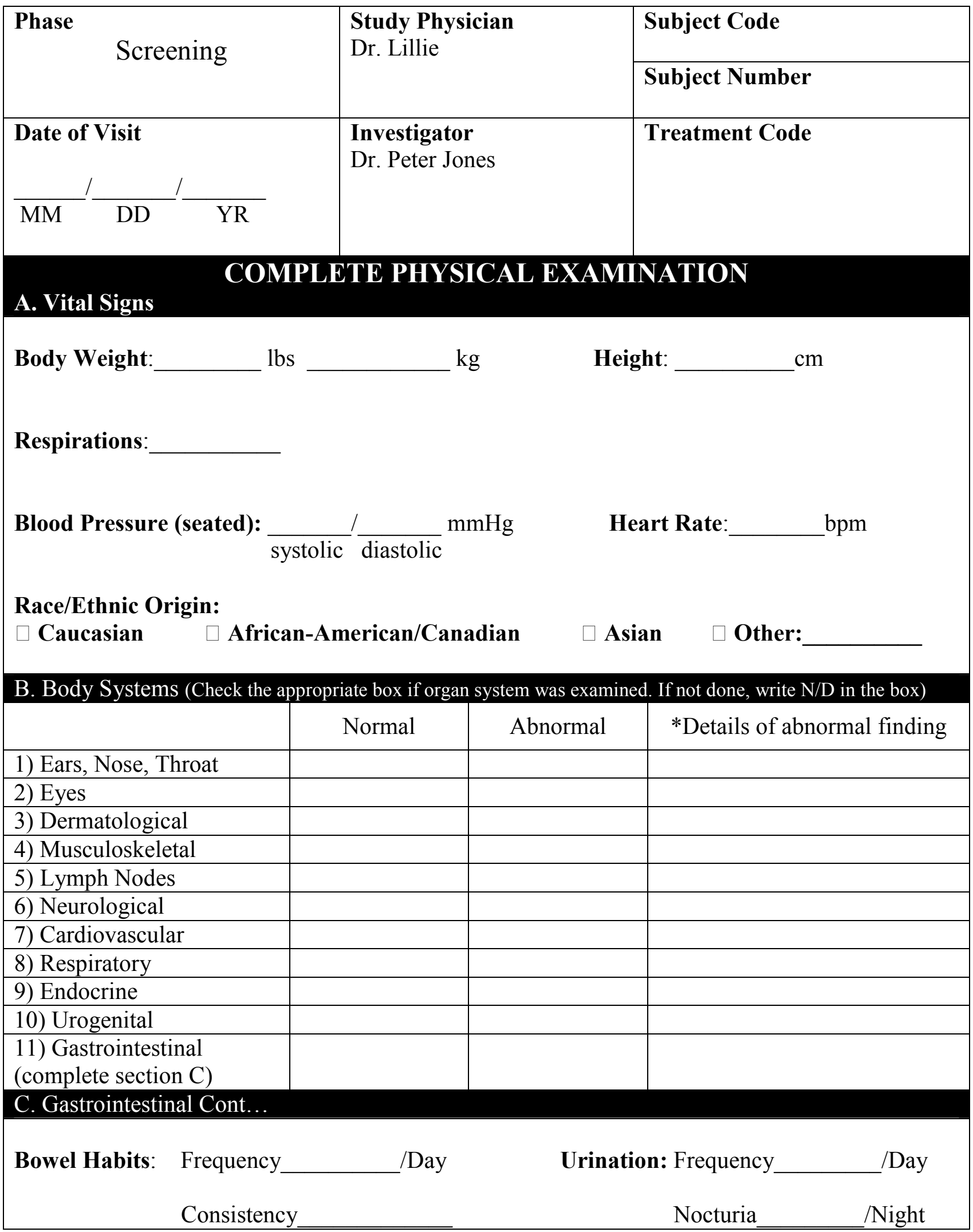




\begin{tabular}{|c|c|c|}
\hline \multicolumn{3}{|l|}{ Medications: } \\
\hline \multicolumn{3}{|l|}{ Hospitalizations: } \\
\hline \multicolumn{3}{|l|}{ Family History: } \\
\hline \multicolumn{3}{|l|}{ D. Inclusion Criteria Screening } \\
\hline (All of the following must be 'YES' for valid entry into the study) & YES & NO \\
\hline \multicolumn{3}{|l|}{$\begin{array}{l}\text { Male subject, aged } 18-60 \text { years old who has given informed written consent } \\
\text { AGE: }\end{array}$} \\
\hline \multicolumn{3}{|l|}{ LDL-cholesterol $>2.5 \mathrm{mmol} / \mathrm{L} \quad$ VALUE: } \\
\hline BMI within the range of $25-36 \mathrm{~kg} / \mathrm{m}^{2}$ & & \\
\hline \multicolumn{3}{|l|}{ Complete blood count (CBC) within normal ranges. } \\
\hline \multicolumn{3}{|l|}{ Blood biochemistry within normal ranges. } \\
\hline Electrocardiogram normal. & & \\
\hline
\end{tabular}

\section{E. Medical History Exclusion Criteria Screening Questionnaire}

(All of the following must be answered 'NO' for valid entry into the study) Have you taken a medication affecting lipid metabolism (cholestyramine, colestipol, niacin, colfibrate, gemfibrozil, probucol, HMG-CoA reductase inhibitors, and high-dose dietary supplements, plant sterols or fish oil capsules) within the past 3 months?

Do you take any natural or pharmaceutical weight loss supplements or products?

Do you have major food allergies or are you sensitive to Asteraceae/Compositae family (e, g., ragweed, marigolds, daisies)?

Do you smoke?

Do you consume large amounts of alcohol?

(more than 2 drinks per day or 12 drinks per week)

Do you have diabetes mellitus? 


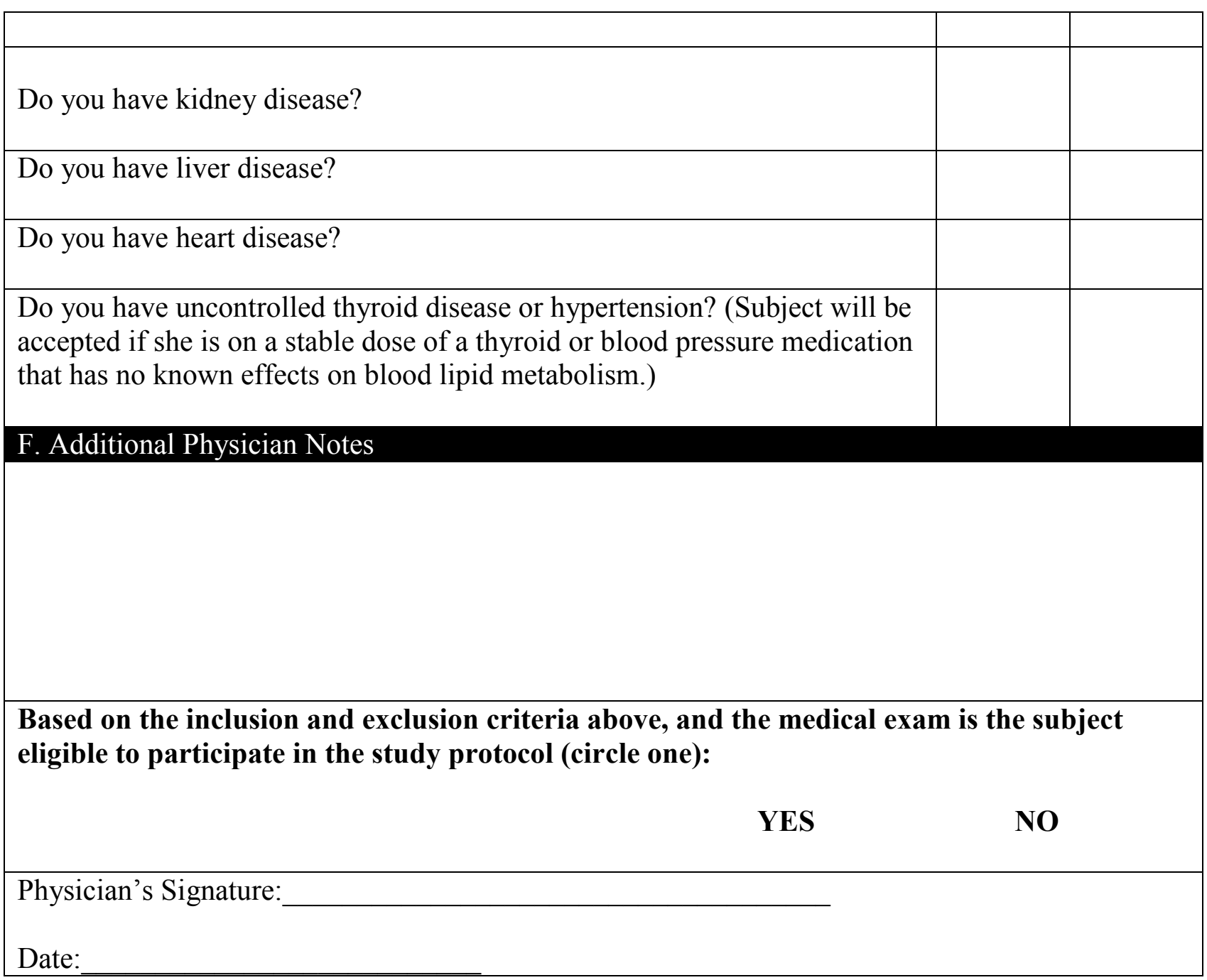




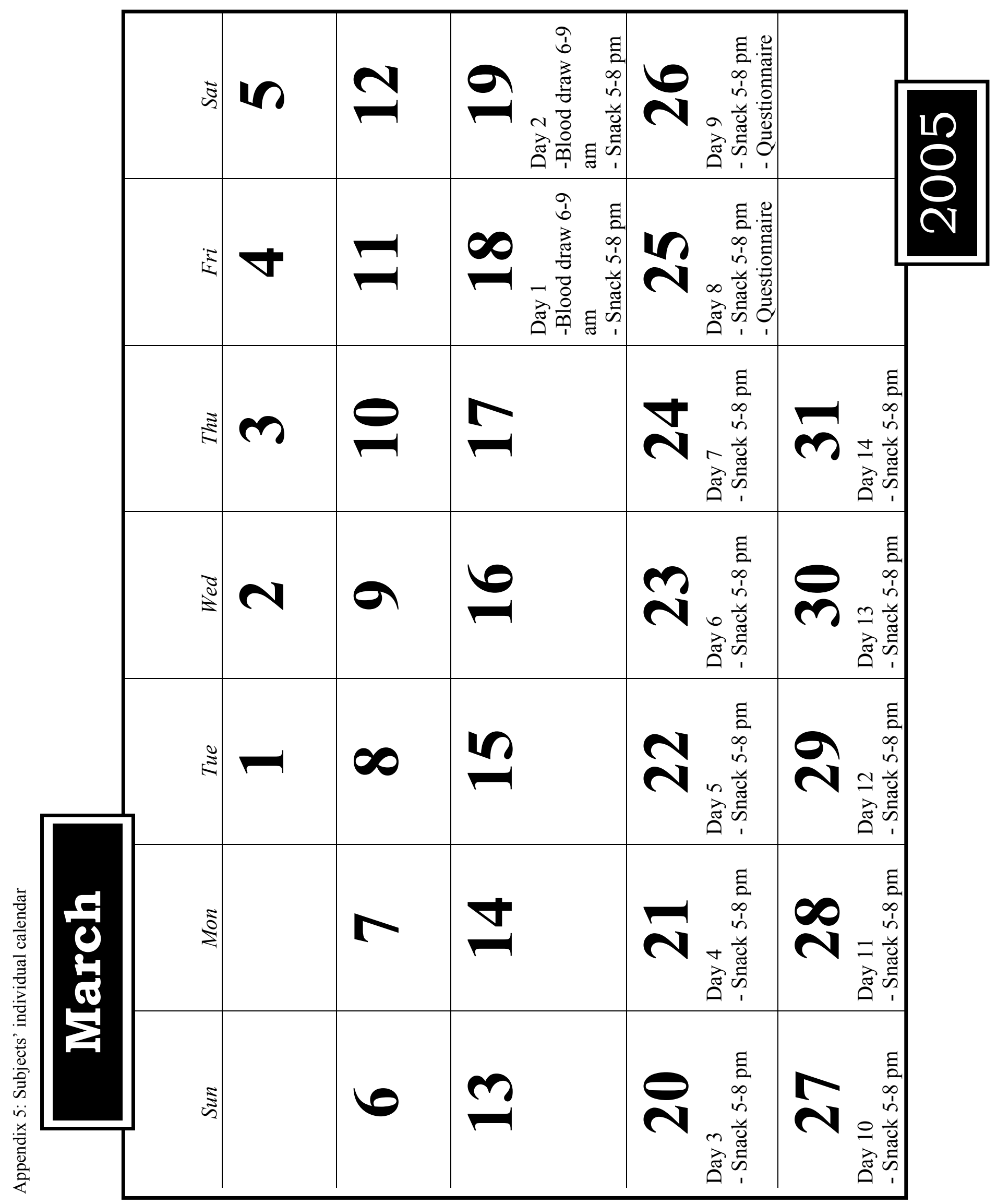


Appendix 6: Subjects' 24-hour recall form

Subject ID:

Date:

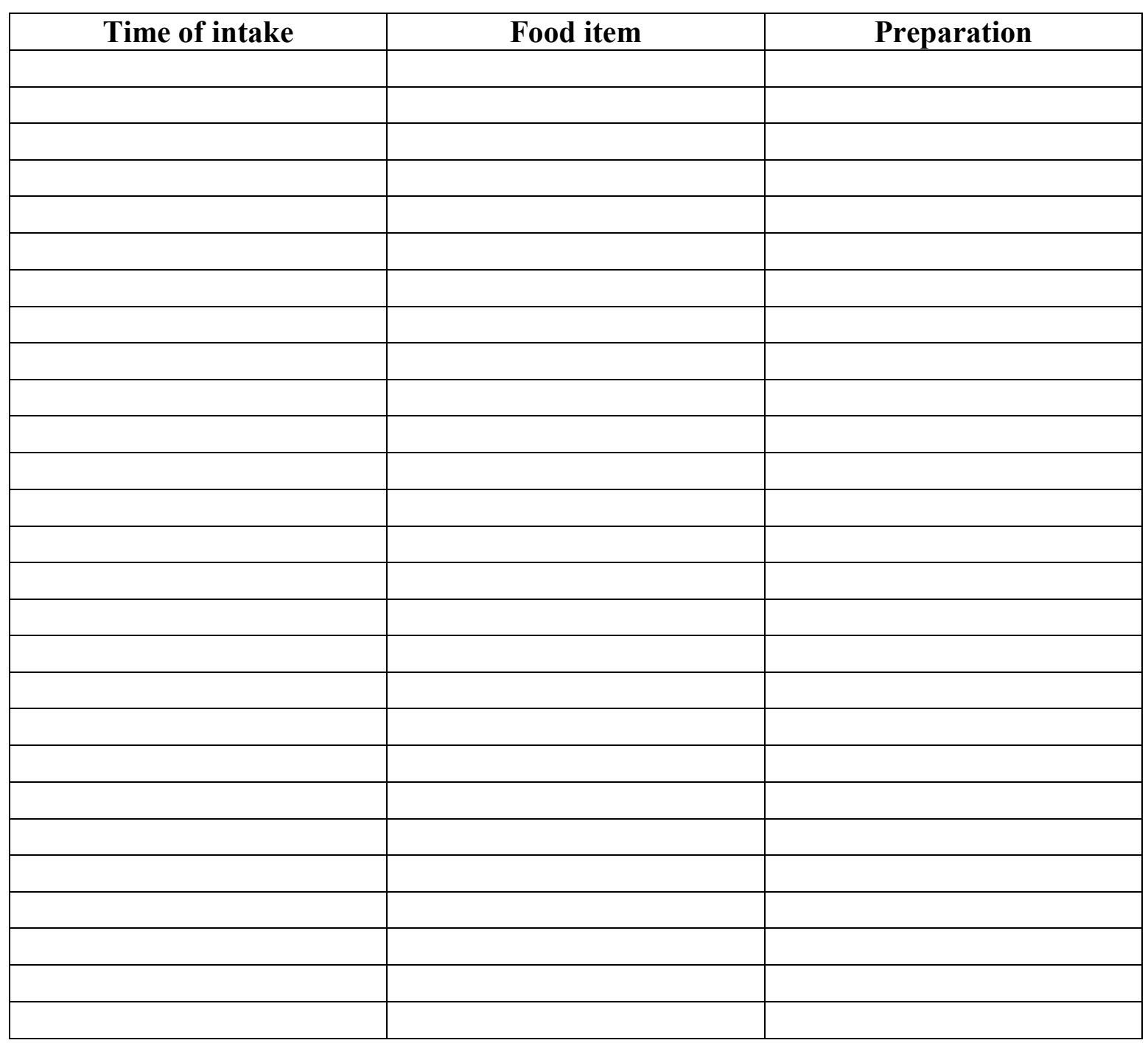


Date:

Dear Mr/Mrs ...,

Please find attached a copy of your results from the two phases of the study entitled "Evaluation of Policosanols as Functional Food Ingredients for Cholesterol-Lowering". The attached report includes your total cholesterol, LDL-cholesterol, HDL-cholesterol and triglyceride values as well as percent changes, in response to the policosanol treatment and the control. This report also provides you with the average percent changes of the group involved in the study, so that you can compare your results to the rest of the subjects.

We would like to thank you for the time and effort you invested in this clinical trial and we hope that you found your participation in this research study to be enriching. Should you require more information concerning your results, please contact us at (514) 3987527.

Sincerely,

Amira Kassis

Peter JH Jones. 
Name :...

ID: 205
Phase 1Policosanol

Phase 2: Control

\section{Total cholesterol}

\begin{tabular}{|l|r|r|c|c|}
\hline Unit: $\mathrm{mmol} / \mathrm{L}$ & \multicolumn{1}{|l|}{ tart } & \multicolumn{1}{l|}{ End } & \% change & \% group change \\
\hline Control (placebo) & 5.55 & 5.22 & -5.86 & -4.08 \\
\hline Policosanol & 5.65 & 5.90 & 4.34 & 1.91 \\
\hline
\end{tabular}

\begin{tabular}{|l|c|c|c|c|}
\hline \multicolumn{5}{|c|}{ LDL-cholesterol (bad cholesterol) } \\
\hline Unit: mmol/L & \multicolumn{1}{|c|}{ Start } & \multicolumn{1}{|c|}{ End } & \% change & \% group change \\
\hline Control (placebo) & 3.85 & 3.45 & -10.39 & -1.51 \\
\hline Policosanol & 3.75 & 3.95 & 5.33 & 4.66 \\
\hline
\end{tabular}

\begin{tabular}{|l|c|c|c|c|}
\hline \multicolumn{5}{|c|}{ HDL-cholesterol (good cholesterol) } \\
\hline Unit: mmol/L & \multicolumn{1}{|c|}{ Start } & \multicolumn{1}{|c|}{ End } & \% change & \% group change \\
\hline Control (placebo) & 1.52 & 1.50 & -0.99 & -7.41 \\
\hline Policosanol & 1.52 & 1.57 & 3.63 & -2.74 \\
\hline
\end{tabular}

\begin{tabular}{|l|c|c|c|c|}
\hline \multicolumn{5}{|c|}{ Triglycerides } \\
\hline Unit: $\mathrm{mmol} / \mathrm{L}$ & \multicolumn{1}{|c|}{ Start } & \multicolumn{1}{l|}{ End } & \% change & \% group change \\
\hline Control (placebo) & 1.15 & 1.20 & 4.37 & -4.26 \\
\hline Policosanol & 1.18 & 1.25 & 5.51 & 3.06 \\
\hline
\end{tabular}




\section{Manuscript 1- Published}

Lack of effect of Cuban sugar cane policosanol on plasma cholesterol in golden Syrian hamsters

Amira N. Kassis, Christopher P.F. Marinangeli, Deepak Jain, Naoyuki Ebine, Peter J.H. Jones

Published in Atherosclerosis 
September 17, 2007

To Dr. Peter Jones

The purpose of this letter is to confirm that Dr. Peter Jones, co-author of the below mentioned manuscripts, agrees to their inclusion in the thesis of Amira Kassis, $\mathrm{PhD}$ candidate.

\section{Manuscripts to be included}

1- Kassis AN, Marinangeli CP, Jain D, Ebine N \& Jones PJ (2006) Lack of effect of sugar cane policosanol on plasma cholesterol in golden Syrian hamsters.

Atherosclerosis. Epub ahead of print

2- Marinangeli CP, Kassis AN, Jain D, Ebine N, Cunnane SC \& Jones PJ (2007)

Comparison of composition and absorption of sugarcane policosanols. Br J Nutr. Feb;97(2):381-8

3- Kassis AN \& Jones PJ (2006) Lack of cholesterol-lowering efficacy of Cuban sugar cane policosanols in hypercholesterolemic persons. Am J Clin Nutr. 2006 Nov;84(5): 10

4- Kassis AN \& Jones PJH. Changes in cholesterol metabolism in response to sugar cane policosanol in hypercholesterolemic individuals. To be submitted.

5- Kassis AN, Jones PJH \& Kubow, S. Lack of effect of Cuban sugar cane policosanols on LDL oxidation in hypercholesterolemic individuals. To be submitted.

6- Jones PJH, Kassis AN \& Marinangeli PF. Policosanol loses its luster as a cholesterol lowering agent.

Manuscript 1: the candidate's role in this study was sharing responsibilities in running the animal trial collecting and analyzing data. The candidate wrote the manuscript with the guidance and feedback from co-authors.

Manuscript 2: the candidate was involved in running the animal trial, collecting data as well as reviewing and providing feedback for the manuscript. 
Manuscript 3,4 and 5: the candidate ran the clinical trial, collected and analyzed data. The manuscripts were written by the candidate with guidance and feedback from co-authors. Manuscript 6: the candidate was involved in writing the manuscript.

I, Peter Jones, co-author of the fore-mentioned manuscripts, hereby agree to their inclusion in the $\mathrm{PhD}$ thesis of the candidate, Amira Kassis..

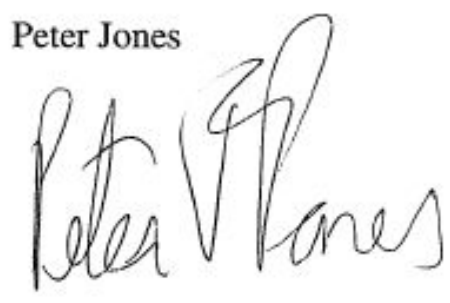


September 17, 2007

To Christopher Marinangeli,

The purpose of this letter is to confirm that Christopher Marinangeli, co-author of the below mentioned manuscripts, agrees to their inclusion in the thesis of Amira Kassis, $\mathrm{PhD}$ candidate.

\section{Manuscripts to be included}

1- Kassis AN, Marinangeli CP, Jain D, Ebine N \& Jones PJ (2006) Lack of effect of sugar cane policosanol on plasma cholesterol in golden Syrian hamsters.

Atherosclerosis. Epub ahead of print

2- Marinangeli CP, Kassis AN, Jain D, Ebine N, Cunnane SC \& Jones PJ (2007)

Comparison of composition and absorption of sugarcane policosanols. Br J Nutr.

Feb;97(2):381-8

3- Jones PJH, Kassis AN, Marinangeli PF. Policosanol loses its luster as a cholesterol lowering agent. To be submitted.

Manuscript 1: the candidate's role in this study was sharing responsibilities in running the animal trial collecting and analyzing data. The candidate wrote the manuscript with the guidance and feedback from co-authors.

Manuscript 2: the candidate was involved in running the animal trial, collecting data as well as reviewing and providing feedback for the manuscript.

Manuscript 3: the candidate was involved in writing the manuscript.

I, Christopher Marinangeli, co-author of the aforementioned manuscripts, hereby agree to their inclusion in the $\mathrm{PhD}$ thesis of the candidate, Amira Kassis.

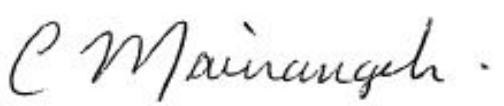

Christopher Marinangeli 


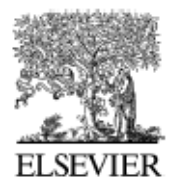

ATHEROSCLEROSIS

ELSEVIER

Atherosclerosis 194 (2007) 153-158

www.elsevier.com/locate/atherosclerosis

\title{
Lack of effect of sugar cane policosanol on plasma cholesterol in golden syrian hamsters
}

\author{
Amira N. Kassis, Christopher P.F. Marinangeli, Deepak Jain, \\ Naoyuki Ebine, Peter J.H. Jones* \\ School of Dietetics and Human Nutrition, McGill University, 21,111 Lakeshore Road, \\ Ste-Anne-de-Bellevue, Montrél, Quebec H9X 3V9, Canada \\ Received 20 July 2006; received in revised form 14 September 2006; accepted 9 October 2006 \\ Available online 22 November 2006
}

\begin{abstract}
Policosanol is a mixture of higher aliphatic alcohols shown to have beneficial effects on plasma lipid levels in animals and humans. Over 50 studies have reported significant reductions in plasma cholesterol using policosanol obtained from Cuban sugar cane (Dalmer, La Havana, Cuba). However, other research groups using policosanol from alternative sources have failed to reproduce the efficacy of these alcohols observed in earlier studies. Therefore, the objective of the present study was to compare the cholesterol-lowering effect of the Dalmer sugar cane policosanol (SCP) product versus an alternative mixture of similar policosanol composition. Forty-eight male Golden Syrian hamsters were randomly assigned to four groups and fed experimental diets ad libitum for a period of 4 weeks: (i) non-cholesterol control, (ii) $0.1 \%$ cholesterol control, (iii) $0.1 \%$ cholesterol diet supplemented with $275 \mathrm{mg} / \mathrm{kg}$ diet of Dalmer Cuban sugar cane policosanol and (iv) $0.1 \%$ cholesterol diet supplemented with $275 \mathrm{mg} / \mathrm{kg}$ diet of alternative sugar cane policosanol. Hamsters were sacrificed and blood was collected at the end of the feeding period. Body weights and food intakes were similar across study groups. Neither of the two policosanol treatments had any significant effect on plasma lipid levels, as compared to cholesterol control. The outcome of the present study questions the clinical usefulness of policosanol mixtures as cholesterol-lowering nutraceuticals.
\end{abstract}

(6) 2006 Elsevier Ireland Ltd. All rights reserved.

Keyworis: Policosanol; Very long chain fatty alcohols; Octacosanol; Cholesterol lowering; Low-density lipoprotein; Hamsters

\section{Introduction}

Sugar cane policosanol (SCP) is a mixture of higher aliphatic alcohols with chain lengths ranging from 24 to 34 carbons, the most important and prevalent of which is octacosanol (C28). Cuban SCP, derived from the waxy coating of stems and leaves of sugar cane and other plant materials, has been shown to exert beneficial effects on lipid profiles in animals and humans in numerous studies conducted by a single laboratory and using an SCP product manufactured by Dalmer laboratories (La Havana, Cuba) [1]. Animal trials testing the alcohols within experimental models including rats, rabbits, dogs and monkeys, have demonstrated reduc-

\footnotetext{
+ Corresponding author at: Tel: + 15143987547 ; fax: +1 5143987739

E-mail address: peter.jones@mcgill.ca (P.J.H. Jones).
}

tions in circulating total cholesterol (TC) levels consistently greater than $13 \%[2-6]$. Similarly, human policosanol feeding trials [7-10] have shown reductions of up to 20 and $30 \%$ in TC and low-density lipoprotein cholesterol (LDL-C) levels, respectively, depending on dose and duration of SCP treatment. However, laboratories external to the original Cuban research group have failed to reproduce the results of original research in studies using hamsters [11], rabbits [12], as well as in human clinical trials on hypercholesterolemic volunteers $[13,14]$. Despite similar octacosanol content, potential explanations for the disparity in results of studies conducted outside of Cuba may lie in the fact that the policosanol mixtures used in these trials differed in minor alcohol content [11-15]. Only two human studies to date have examined the effects of the Dalmer policosanol mixture on the lipid profile $[16,17]$. No significant changes in TC and LDL-C 
levels were observed. Therefore, the similarity of the composition of policosanol mixtures to the Dalmer mixture shown to reduce blood cholesterol remains at the core of the controversy regarding policosanol lipid-lowering action.

The objective of the present study was to compare the lipid-lowering effect of the Dalmer Cuban product (SCP1) and an alternative mixture (SCP2) in the golden Syrian hamster, one used routinely in studies of dietary lipid effects. It was hypothesized that there would be no difference between the effect of SCP1 and SCP2 on plasma lipids.

\section{Materials and methods}

\subsection{Animals and diets}

Two-week old male golden Syrian hamsters $(n=48)$ weighing $80-120 \mathrm{~g}$ were housed in individual cages with free access to water and were subjected to a 12-h light:12-h dark cycle. Hamsters were fed regular rodent chow ad libitum for a 2-week acclimatization period before being randomly assigned to one of four different diets, including (i) noncholesterol control (NCC), (ii) $0.1 \%$ cholesterol control (CC), (iii) $0.1 \%$ cholesterol diet supplemented with $275 \mathrm{mg} / \mathrm{kg}$ diet of SCP1 and (iv) $0.1 \%$ cholesterol diet supplemented with $275 \mathrm{mg} / \mathrm{kg}$ diet of SCP2 (Degussa Bioactives, Germany). The composition of the animal feed is detailed in Table 1. The four diets were adapted from the American Institute of Nutrition (AIN) 93-G formula of a purified diet for experimental rodents and had similar nutrient compositions [18]. SCP pills were crushed and mixed thoroughly with the fat component of the diet prior to incorporation in the rest of the mixture. The alcohol composition of the SCP1 brand was 66-67\% octacosanol, and $33-34 \%$ of other alcohols [1]. The composition of the SCP2 brand was $68.8 \%$ octacosanol and $31.2 \%$ of other alcohols (Degussa Bioactives product information).

\subsection{Protocol}

Hamsters were systematically randomized according to their body weights and assigned to the four experimental diets for a period of 28 days. Each of the four diet groups consisted of 12 hamsters. During randomization, animals were divided into six blocks of eight hamsters on the four different treatments. This randomization procedure ensured a good distribution of body weights on the different treatments administered.

Body weights were recorded once a week while food intake was measured every 3 days. Average intakes were calculated by subtracting leftovers and spillage from the food portion given to the animals. On day 28 , hamsters were sacrificed by decapitation after carbon dioxide inhalation and trunk blood was collected in EDTA tubes and placed on ice. Blood was then centrifuged and plasma was separated and stored at $-20^{\circ} \mathrm{C}$.

\subsection{Plasma lipid analysis}

Blood samples from day 28 were used to determine lipid profiles. Plasma TC, high-density lipoprotein cholesterol (HDL-C) and triglyceride (TG) concentrations were analysed with enzymatic kits, standardized reagents, and standards using a VP Autoanalyzer (Abbott Laboratories, North Chicago, IL, USA). Non-HDL-C (N-HDL-C) was calculated for hamsters using the following equation: (N-HDL$\mathrm{C})=(\mathrm{TC})-(\mathrm{HDL}-\mathrm{C})$.

\subsection{Statistical analysis}

Sample size $(n=48)$ was calculated to provide a power of $80 \%$ based on the smallest effect size on plasma TC seen in previous literature. The effect of diet groups on TC, HDL$\mathrm{C}$ and TG was examined using a one-way ANOVA with a

Table 1

Composition of the hamster diet

\begin{tabular}{|c|c|c|c|c|}
\hline \multirow{2}{*}{ Constituents (wt. \%) } & \multicolumn{4}{|c|}{ Diet groups } \\
\hline & $\mathrm{NCC}(\%)$ & $\mathrm{CC}(\%)$ & $\operatorname{SCP} 1(\%)$ & $\operatorname{SCP} 2(\%)$ \\
\hline Casein & 20.0 & 20.0 & 20.0 & 20.0 \\
\hline Sucrose & 33.2 & 33.2 & 33.0 & 33.1 \\
\hline Beef tallow/safflower oil mix & 10.0 & 10.0 & 10.0 & 10.0 \\
\hline Cellulose & 5.0 & 5.0 & 5.0 & 5.0 \\
\hline Vitamin mixture & 1.0 & 1.0 & 1.0 & 1.0 \\
\hline Choline bitartrate & 0.2 & 0.2 & 0.2 & 0.2 \\
\hline Butylated hydroxytoluene & 0.0 & 0.0 & 0.002 & 0.002 \\
\hline Cholesterol & 0.0 & 0.1 & 0.1 & 0.1 \\
\hline Dalmer policcsanol powder ${ }^{\mathrm{a}}$ & - & - & 0.5 & - \\
\hline Degussa policosanol powder $r^{b}$ & - & - & & 0.03 \\
\hline
\end{tabular}

NCC, non-cholesterol control; CC, cholesterol control; SCP1, authentic Cuban sugar cane policosanols; SCP2, altemative sugar cane policosanols.

a Purity of Dalmer policosanol product $=6.0 \%$.

${ }^{b}$ Purity of Degussa policosanol product $=92.0 \%$. 
statistical significance set at $p<0.05$. The block effect was included as part of the statistical model to rule out any effect of age on blood lipids. Additionally, effects of body weight and food intake on plasma lipids were tested as covariates to the effect of policosanol treatments. All data are presented as mean \pm S.E.M.

\section{Results}

No behavioural or physical abnormalities were recorded in hamsters throughout the trial period. None of the animals died nor escaped during the experiment.

\subsection{Growth of hamsters across the study period}

Weight patterns across the study period are illustrated in Fig. 1. Average initial weights of hamsters in the NCC, CC, SCP1 and SCP2 groups were 109.6 \pm 0.64 . $109.7 \pm 0.64,109.6 \pm 0.64$ and $109.4 \pm 0.88 \mathrm{~g}$, respectively. Hamster growth was higher in the first three weeks and decreased towards the end of the feeding period. Body weights in the NCC, CC, SCP1 and SCP2 groups increased by $17.41,17.6,19.2$ and $15.7 \%$, respectively, as compared to baseline. No significant differences were observed amongst groups for weight changes across the feeding period. Endpoint average weights in the NCC, CC, SCP1 and SCP2 groups were $126.4 \pm 1.82,129.0 \pm 1.82,130.3 \pm 2.05$ and $125.4 \pm 2.35 \mathrm{~g}$, respectively. When compared across groups, body weights recorded each feeding week did not differ significantly.

\subsection{Food intake of hamsters throughout the feeding period}

Average food intake of animals throughout the feeding period was $7.68 \pm 0.19,7.82 \pm 0.18,7.59 \pm 0.21$ and

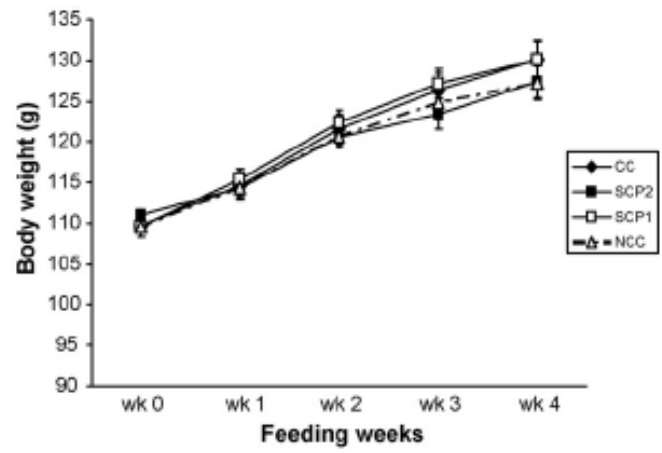

Fig. 1. Body weights (mean \pm S.E.M.) across the feeding period. NCC, noncholesterol control; CC, cholesterol control; SCP1, Dalmer Cuban sugarcane policosanol; SCP2, altemative sugar cane policosanol; $n=44$. No significant difference in weight amongst the four groups at the different time points.

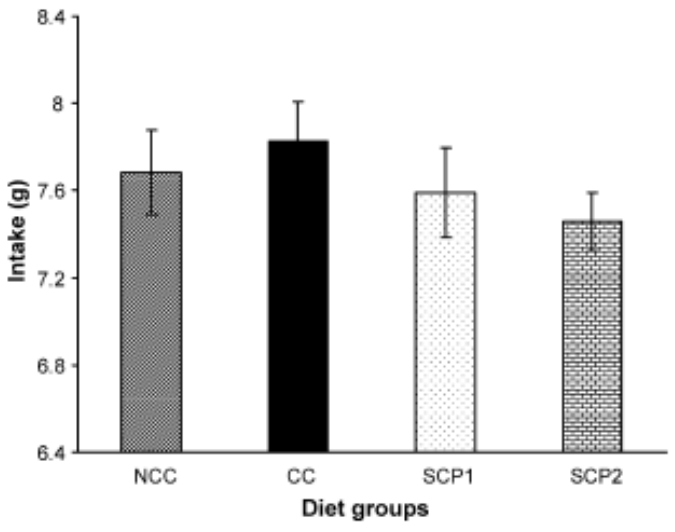

Fig. 2. Average intake of hamsters in different diet groups throughout the study period. NCC, non-cholesterol control; CC, cholesterol control; SCP1, Dalmer Cuban sugar cane policosanol: SCP2, alternative sugar cane poli$\operatorname{cosanol} ; n=44$. No significant difference in average intake amongst the four groups.

$7.46 \pm 0.13 \mathrm{~g}$ in the NCC, CC, SCP1 and SCP2, respectively, as presented in Fig. 2. Average food intakes did not differ significantly across the four diet groups. The daily amounts of policosanol ingested by the $\mathrm{SCP} 1$ and $\mathrm{SCP} 2$ treatment groups were 20.1 and $20.5 \mathrm{mg} / \mathrm{kg}$ body weight, respectively.

\subsection{Growth and intake of hamsters versus plasma lipids}

Body weight of hamsters recorded every week throughout the study period was not significantly correlated with plasma TC, HDL-C and TG values. Similarly, there was no association of daily feed intake with plasma lipids in hamsters.

\subsection{Lipid values as a result of policosanol feeding}

Plasma TC, HDL-C, N-HDL-C and TG endpoint values across treatments and percent differences from control groups are outlined in Table 2. There was no significant difference between the effect of SCP1 and SCP2 administration on plasma TC endpoints. Lower TC values were recorded in hamsters consuming the policosanol diets as compared to the CC group, however, these differences were not statistically significant.

Similarly to TC, calculated N-HDL-C values were not significantly different in the SCP1 and SCP2 groups. Also, when compared to $\mathrm{CC}$, the 5.3 and $9.6 \%$ reductions in $\mathrm{N}$ HDL-C as a result of SCP1 and SCP2 treatments were not statistically significant. Endpoints in the policosanol groups were higher than in the NCC group, however non-significant.

HDL-C and TG endpoints were not significantly affected by the SCP1 and SCP2 treatments as compared to the CC group. However, when compared to the NCC group, mean plasma TG in hamsters on the SCP1 diet was $68 \%$ higher than the mean in the NCC group. 
Table 2

Plasma lipids (mean \pm S.E.M.) in response to policosanol diets as compared to controls

\begin{tabular}{lrcc}
\hline & $\begin{array}{l}\text { Concentrations } \\
(\mathrm{mmol} / \mathrm{L})\end{array}$ & $\begin{array}{l}\text { Change from } \\
\text { NCC }(\%)\end{array}$ & $\begin{array}{c}\text { Change from } \\
\text { CC }(\%)\end{array}$ \\
\hline Total cholesterol & & & \\
NCC & $4.78 \mathrm{a} \pm 0.21$ & - & -24.4 \\
CC & $6.33 \mathrm{~b} \pm 0.21$ & 32.4 & - \\
SCP1 & $5.80 \mathrm{~b} \pm 0.21$ & 21.3 & -8.37 \\
SCP2 & $5.67 \mathrm{a}, \mathrm{b} \pm 0.28$ & 18.6 & -10.4 \\
HDL-cholesterol & & & \\
NCC & $2.60 \mathrm{a} \pm 0.17$ & - & -15.9 \\
CC & $3.09 \mathrm{a} \pm 0.17$ & 18.8 & - \\
SCP1 & $2.73 \mathrm{a} \pm 0.17$ & 5.00 & -11.6 \\
SCP2 & $2.74 \mathrm{a} \pm 0.23$ & 5.38 & -11.3 \\
Non-HDL-cholesterol & & -32.7 \\
NCC & $2.18 \mathrm{c} \pm 0.28$ & - & - \\
CC & $3.24 \mathrm{~d} \pm 0.28$ & 48.6 & -5.3 \\
SCP1 & $3.07 \mathrm{c}, \mathrm{d} \pm 0.28$ & 40.8 & -9.6 \\
SCP2 & $2.93 \mathrm{c}, \mathrm{d} \pm 0.36$ & 34.4 & \\
Triglycerides & & & -25.4 \\
NCC & $2.38 \mathrm{a} \pm 0.28$ & - & - \\
CC & $3.19 \mathrm{a}, \mathrm{b} \pm 0.28$ & 34.0 & 6.9 \\
SCP1 & $4.00 \mathrm{~b} \pm 0.36$ & 68.1 & 43.3 \\
SCP2 & $3.41 \mathrm{a}, \mathrm{b} \pm 0.28$ & & \\
\hline
\end{tabular}

NCC, non-cholesterol control; CC, cholesterol control: SCPI, authentic Cuban sugar cane policosanols; SCP2, alternative sugar cane policosanols; $n=44$. Means within a lipid category with different letters ( $\mathrm{a}$ and $\mathrm{b}$ ) are statistically significant at $p<0.01$. Means within a lipid category with different letters (c and d) are statistically significant at $p<0.05$.

\section{Discussion}

Results of the present study show, for the first time, that neither the Dalmer Cuban nor the alternative Degussa policosanol mixtures possess any significant effect on plasma TC, HDL-C and TG levels over the moderate term in the hamster model. These results are in contrast with previous research conducted on the Dalmer mixture that demonstrates reductions in total cholesterol and LDL-cholesterol of more than $13 \%$ [19]. Studies conducted on rabbits, dogs and monkeys agree with the conclusion that policosanol reduces levels of LDL-C and TC significantly at dosages ranging from 5 to $500 \mathrm{mg} / \mathrm{kg}$ body weight $/$ day [2-6]. The hamster model used in the present study is considered to be one of the most suitable models for studying lipid lowering mechanisms [20] and has shown to resemble humans with regard to regulation of LDL-C metabolism [21]. One could argue that the absence of effect on N-HDL-C could be accounted for by the fact that hamsters carry a larger proportion of plasma cholesterol in HDL versus non-HDL particles. Nonetheless, we would have expected to see changes in TC and N-HDL-C similar to those seen in numerous studies examining the effect of LDL-C lowering substances in hamsters including phytosterols, statins and others [22-25].

Our results agree with the study conducted in 2003 by Wang et al. [11], which compared the efficacy of rice and non-Dalmer SCP to that of plant sterols, both given in the context of a hypercholesterolemic diet. Hamsters in the policosanol groups did not show changes in TC and N-HDL-C as compared to cholesterol control, while the plant sterol group demonstrated TC levels significantly lower than control and policosanol groups by 22 and $30 \%$, respectively. Our results also agree with another study conducted in 2004 by Murphy and Howe [12], not using the hamster model, but providing rabbits with an alternative mixture of SCP, and showing no shifts in plasma lipids. In the current study, inclusion of the NCC group in the analysis ensured that our hamster model did respond to dietary intervention. In fact, this was manifested by a significant reduction in plasma TC and N-HDL-C as compared to the $\mathrm{CC}$ group.

Studies on the effect of SCP supplementation on cholesterol metabolism have established that cholesterol-lowering effects, mainly mediated by a decrease in serum LDL-C levels, were correlated with the inhibition of hepatic cholesterol synthesis $[6,26,27]$ and the stimulation of receptor-mediated LDL uptake by the liver $[5,6]$. It could therefore be argued that the absence of effect shown in the study by Wang et al. was partly caused by the high cholesterol content of the diet $(0.25 \%$ by weight), which could have maximally suppressed cholesterol biosynthesis. Although statins, which also impact cholesterol synthesis, were shown to be effective in the context of a high cholesterol diet [28], this issue remains a point of concern in terms of the effect of dietary cholesterol on cholesterol biosynthesis [29]. Consequently, the proportion of cholesterol used in the feed for the present study was $0.1 \%$ by weight, in order to reduce the suppression of cholesterol biosynthesis potentially masking the effect of policosanol. This choice of cholesterol content was also made to approach a human cholesterol intake.

Cholesterol synthesis measurements were conducted using ${ }^{14} \mathrm{C}$ acetate in the in vitro studies mentioned above $[26,27]$. The authors have attributed the reduction in ${ }^{14} \mathrm{C}$ concentration in fibroblasts treated with SCP to their inhibitory effect on the conversion of acetate to mevalonate leading eventually to a decrease in cholesterol synthesis [26,27]. In 2006, the same method was used by Singh and $\mathrm{Li}[30]$ in an in vitro study examining the effect of a policosanol formulation on cholesterol synthesis in human fibroblasts. The authors were able to observe a $30 \%$ reduction in cholesterol labeling, agreeing with results obtained by the original research group. However, adding labeled acetate to a cell medium containing policosanol may lead to the dilution of labeled acetate as a result of the degradation of policosanol into its metabolites, namely acetate itself, therefore falsely indicating a reduction of cholesterol synthesis at the level of acetate incorporation. It is thus possible that the methods used to date may have been inappropriate for the determination of policosanol metabolism and its impact on cholesterol synthesis. In fact, the newer independent human studies clearly showing that policosanol from Cuba or other sources has no true effect on the lipid profile of hypercholesterolemic humans [15-17,31-33] support the hypothesis that the suppression observed in vitro is artificial. 
The composition of alternative policosanol mixtures used by external research groups in most animal and human studies raised criticism in terms of the efficacy of any composition that diverges from the original Cuban mixture. Although the main active compound octacosanol is found in similar amounts in both Dalmer and alternative mixtures (between 60 and $70 \%$ ), products with different alcohol compositions appear to have different lipid-lowering activities despite their similar octacosanol contents [34]. In order to avoid the limitations pertaining to product authenticity, the present study used the original Cuban mixture manufactured by Dalmer laboratories in La Havana, Cuba, and compared it to an alternative policosanol mixture. Nevertheless, our data shows that hamsters responded to neither treatment, reinforcing the conclusions seen in studies showing no effect. In fact, our results agree with two recent human studies conducted in independent laboratories using the Dalmer product and reporting no change in TC and LDL-C in hypercholesterolemic individuals $[16,17]$.

The outcomes of the present trial should not be overinterpreted without considering the differences in methodology between this study and animal studies showing positive results. The effect of policosanol on lipid metabolism might differ amongst different animal species, therefore, the use of the hamster model in this trial might account for the lack of effect of policosanol, as compared to other studies using species such as rats and rabbits $[5,6]$. However, noting that hamsters resemble humans in terms of LDL metabolism, data obtained using this model should be more applicable to humans than would be the case using some other models.

In most animal trials showing positive outcomes, hypercholesterolemia was induced endogenously through enhancement of cholesterol biosynthesis using a fat-free wheat starch-casein diet. In the present trial, hypercholesterolemia was induced exogenously through the addition of $0.1 \%$ cholesterol in the animals' diet. Since policosanol are believed to act at the level of cholesterol synthesis in the liver $[6,26,27]$, the difference in the baseline biosynthesis induced by the diets could have accounted for the variation in results. Again, the diet used in the present study is more easily applicable to a human situation as compared to a diet free of fat and cholesterol.

In conclusion, the results of the present study show that both Dalmer Cuban SCP and alternative mixtures such as Degussa SCP, fail to reduce plasma lipid levels in hamsters. These outcomes question the efficacy of policosanol as a lipid-lowering agent and emphasize the need to explore mechanisms underlying its effect on cholesterol biosynthesis and absorption.

\section{Acknowledgment}

This study was supported by the Advanced Foods and Materials network—Networks of Centres of Excellence.

\section{References}

[1] Mas R. Monographs-policosanol: hypolipidemic, antioxidant, treatment of atherosclerosis. Drugs Future 2000:25:569-\$6.

[2] Amuzazabala MLCD, Mas R, Molina V, Valdes S, Laguna A. Cholesterol-lowering effects of policosanol in rabbits. Biol Res 1994:27:205-8.

[3] Mesa AR, Mas R, Noa M, et al. Toxicity of policosanol in beagle dogs: one-year study. Toxicol Lett 1994;73(2):81-90.

[4] Rodriguez-Echenique CMR, Mas R. Noa M, et al. Effects of policosanol chronically administered in male monkeys (Macaca arctoides). Food Chem Toxicol 1994;32:565-75.

[5] Menendez R, Amor AM, Gonzalez RM, Fraga V, Mas R. Effect of policosanol on the hepatic cholesterol biosynthesis of normocholesterolemic rats. Biol Res 1996;29(2):253-7.

[6] Menendez R, Arruzazabala L, Mas R, et al. Cholesterol-lowering effect of policosanol on rabbits with hypercholesterolaemia induced by a wheat starch-casein diet. Br J Nutr 1997;77(6):92332.

[7] Menendez R, Mas R, Amor AM, et al. Effects of policosanol treatment on the susceptibility of low density lipoprotein (LDL) isolated from healthy volunteers to oxidative modification in vitro. $\mathrm{Br} \mathrm{J}$ Clin Pharmacol 2000:50(3):255-62.

[8] Castano G, Mas R, Fernandez L, et al. Effects of policosanol 20 versus $40 \mathrm{mg} / \mathrm{day}$ in the treatment of patients with type II hypercholesterolemia: a 6-month double-blind study. Int J Clin Phamacol Res 2001:21(1):43-57.

[9] Mirkin A, Mas R, Martinto M, et al. Efficacy and tolerability of policosanol in hypercholesterolemic postmencpausal women. Int $\mathrm{J}$ Clin Pharmacol Res 2001:21(1):31-41.

[10] Castano GMR, Femández L, Illnait J, et al. A randomized, doubleblind, placebo-controlled study of the efficacy and tolerability of policosanol in adolescents with type II hypercholesterolemia. Curr Ther Res 2002;63:286-303.

[11] Wang YW, Jones PJ, Pischel I, Fairow C. Effects of policosanols and phytosterols on lipid levels and cholesterol biosynthesis in hamsters. Lipids 2003;38(2): $165-70$.

[12] Murphy KJSD, Howe PR. Lack of effect of sugar cane and sunflower seed policosanols on plasma cholesterol in rabbits. Asia Pac J Clin Nutr 2004:13:S69.

[13] Lin YRM, van der Wielen RP, Trautwein EA, et al. Wheat germ policosanol failed to lower plasma cholesterol in subjects with normal to mildly elevated cholesterol concentrations. Metabolism 2004:53:1309-14

[14] Reiner ZT-RE, Romic Z. Effects of rice policosanol on serum lipoproteins, homocysteine, fibrinogen and C-reactive protein in hypercholesterolaemic patients. Clin Drug Imvest 2005;5:701-7.

[15] Greyling A, De Witt C, Oosthuizen W, Jerling JC. Effects of a policosanol supplement on serum lipid concentrations in hypercholesterolaemic and heterozygous familial hypercholesterolaemic subjects. $\mathrm{Br} \mathrm{J}$ Nutr 2006;95:968-75.

[16] Berthold HK. Unverdorben S. Degenhardt R. Bulitta M. GouniBerthold I. Effect of policosanol on lipid levels among patients with hyperchol esterolemia orcombined hyperlipidemia. A randomized controlled trial. JAMA 2006:295(19):2263-9.

[17] Kassis AN, Jones PJ. Lack of cholesterol-lowering efficacy of Cuban sugar cane policosanols in hypercholesterolemic persons. Am J Clin Nutr 2006:84:1003-8.

[18] Reeves PG. Components of the AIN-93 diets as improvements in the AIN-76A diet. J Nutr 1997;1 27(5 Suppl.):838S-41S.

[19] Varady KA, Wang Y, Jones PJ. Role of policosanols in the prevention and treatment of cardiovascular disease. Nutr Rev 2003;61(11):37683.

[20] Kris-Etherton PM. Dietschy J. Design criteria for studies examining individual fatty acid effects on candiovascular disease risk factors: human and animal studies. Am J Clin Nutr 1997;65(5 Suppl.): $1590 \mathrm{~S}-6 \mathrm{~S}$ 
[21] Spady DK, Dietschy JM. Interaction of dietary cholesterol and triglycerides in the regulation of hepatic low density lipoprotein transport in the hamster. J Clin Invest 1988;81(2):300-9.

[22] Otto J, Ordovas JM, Smith D, et al. Lovastatin inhibits diet induced atherosclerosis in FIB golden Syrian hamsters. Atherosclerosis 1995; 114(1):19-28.

[23] Field FJ, Born E, Mathur SN. Stanol esters decrease plasma cholesterol independently of intestinal $\mathrm{ABC}$ sterol transporters and Niemann-Pick Cl-like 1 protein gene expression. J Lipid Res 2004;45(12):2252-9.

[24] Ebine N, Jia X, Demonty I, Wang Y, Jones PJ. Effects of a watersoluble phytostanol ester on plasma cholesterol levels and red blood cell fragility in hamsters. Lipids 2005;40(2): 175-80.

[25] Doggrell SA. Berberine-a novel approach to cholesterol lowering. Exp Opin Invest Drugs 2005;14(5):683-5.

[26] Menendez R, Femandez SI, Del Rio A, et al. Policosanol inhibits cholesterol biosynthesis and enhances low density lipoprotein processing in cultured human fibroblasts. Biol Res 1994:27(3-4):199203.

[27] Menendez R, Amor AM, Rodeiro I, et al. Policosanol modulates HMG-CoA reductase activity in cultured fibroblasts. Arch Med Res 2001;32(1):8-12.

[28] Kohayashi M, Ishida F, Takahashi T, et al. Preventive effect of MK-733 (simvastatin), an inhibitor of HMG-CoA reductase, on hypercholes- terolemia and atherosclerosis induced by cholesterol feeding in rabbits. Jpn J Pharmacol 1989;49(1):125-33.

[29] Jones PJ, Pappu AS, Hatcher L, et al. Dietary cholesterol feoting suppresses human cholesterol synthesis measured by deuterium incorporation and urinary mevalonic acid levels. Arterioscler Thromb Vasc Biol 1996;16(10):1222-8.

[30] Singh DV, Li LTPP. Policosanol inhibits cholesterol synthesis in hepatoma cells by activation o AMP-kinase. J Pharmacol Exp Ther 2006:0 [jpet.106.107144].

[31] Lin Y, Rudrum M, van der Wielen RP, et al. Wheat germ policosanol failed to lower plasma cholesterol in subjects with nomal to mildly elevated cholesterol concentrations. Metabolism 2004;53(10):1309-14.

[32] Reiner Z. Tedeschi-Reiner E. Romic Z. Effects of nice policosanol on serum lipoproteins, homocysteine, fibrinogen and C-reactive protein in hypercholesterolaemic patients. Clin Drug Invest 2005;5:701-7.

[33] Todeschi-Reiner E. Reiner Z. Romic Z, Ivankovic D. A randomized, double-blind, placebo-controlled study of the antilipemic efficacy and tolerability of food supplement policosanol in patients with moderate hypercholesterolemia. Lijec Vjesn 2005;127:273-9.

[34] Castano G, Fernandez L, Mas R, et al. Comparison of the efficacy, safety and tolerability of original policosanol versus other mixtures of higher aliphatic primary alcchols inpatients with type II hypercholesterolemia. Int J Clin Phamacol Res 2002;22(2): 55-66. 


\section{Manuscript 2- Published}

Comparison of composition and absorption of sugarcane policosanols

Christopher P.F. Marinangeli, Amira N. Kassis, Deepak Jain, Naoyuki Ebine, Stephen C.

Cunnane, Peter J.H. Jones

Published in the British Journal of Nutrition 
Dr Amira Kassis

c/o Christopher Marinangeli

Richardson Center for Functional Foods and

Nutraceuticals

196 Innovation Drive

SmartPark, University of Manitoba

Winnipeg, Manitoba

R3T 6C5

Canada

August 30,2007
The Edinburgh Building

Shaftesbury Road

Cambridge CB2 8RU, UK

www.cambridge,org

Telephone $+44(0) 1223312393$

Fax $+44(0) 1223315052$

Email information@cambridge.org

Dear Dr Amira Kassis

Christopher P. F. Marinangeli, Amira N. Kassis, Deepak Jain, Naoyuki Ebine, Stephen C. Cunnane and Peter J. H. Jones, "Comparison of composition and absorption of sugarcane policosanols", British Journal of Nutrition, Volume 97(02): pp 381-388, (2007).

Thank you for your recent permission request to include the above extract(s)

in:

your forthcoming PhD thesis.

Non-exclusive permission is granted free of charge for this specific use on the understanding that you have checked that we do not acknowledge another source for this material.

Please ensure full acknowledgement (author, title, publication date, name of journal and Cambridge University Press).

Yours sincerely

S. Slactrina

Svetlana Shadrina

Publishing Assistant

email sshadrina@cambridge.org 
September 17, 2007

To Dr. Peter Jones

The purpose of this letter is to confirm that Dr. Peter Jones, co-author of the below mentioned manuscripts, agrees to their inclusion in the thesis of Amira Kassis, $\mathrm{PhD}$ candidate.

\section{Manuscripts to be included}

1- Kassis AN, Marinangeli CP, Jain D, Ebine N \& Jones PJ (2006) Lack of effect of sugar cane policosanol on plasma cholesterol in golden Syrian hamsters.

Atherosclerosis. Epub ahead of print

2- Marinangeli CP, Kassis AN, Jain D, Ebine N, Cunnane SC \& Jones PJ (2007)

Comparison of composition and absorption of sugarcane policosanols. Br J Nutr. Feb;97(2):381-8

3- Kassis AN \& Jones PJ (2006) Lack of cholesterol-lowering efficacy of Cuban sugar cane policosanols in hypercholesterolemic persons. Am J Clin Nutr. 2006 Nov;84(5):10

4- Kassis AN \& Jones PJH. Changes in cholesterol metabolism in response to sugar cane policosanol in hypercholesterolemic individuals. To be submitted.

5- Kassis AN, Jones PJH \& Kubow, S. Lack of effect of Cuban sugar cane policosanols on LDL oxidation in hypercholesterolemic individuals. To be submitted.

6- Jones PJH, Kassis AN \& Marinangeli PF. Policosanol loses its luster as a cholesterol lowering agent.

Manuscript 1: the candidate's role in this study was sharing responsibilities in running the animal trial collecting and analyzing data. The candidate wrote the manuscript with the guidance and feedback from co-authors.

Manuscript 2: the candidate was involved in running the animal trial, collecting data as well as reviewing and providing feedback for the manuscript. 
Manuscript 3,4 and 5: the candidate ran the clinical trial, collected and analyzed data. The manuscripts were written by the candidate with guidance and feedback from co-authors. Manuscript 6: the candidate was involved in writing the manuscript.

I, Peter Jones, co-author of the fore-mentioned manuscripts, hereby agree to their inclusion in the $\mathrm{PhD}$ thesis of the candidate, Amira Kassis..

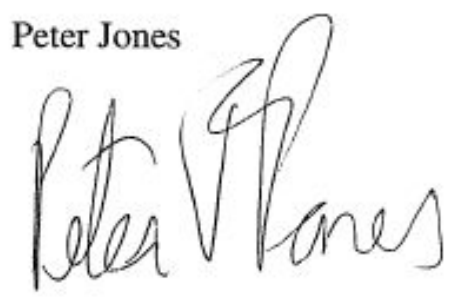


September 17, 2007

To Christopher Marinangeli,

The purpose of this letter is to confirm that Christopher Marinangeli, co-author of the below mentioned manuscripts, agrees to their inclusion in the thesis of Amira Kassis, $\mathrm{PhD}$ candidate.

\section{Manuscripts to be included}

1- Kassis AN, Marinangeli CP, Jain D, Ebine N \& Jones PJ (2006) Lack of effect of sugar cane policosanol on plasma cholesterol in golden Syrian hamsters.

Atherosclerosis. Epub ahead of print

2- Marinangeli CP, Kassis AN, Jain D, Ebine N, Cunnane SC \& Jones PJ (2007)

Comparison of composition and absorption of sugarcane policosanols. Br J Nutr. Feb;97(2):381-8

3- Jones PJH, Kassis AN, Marinangeli PF. Policosanol loses its luster as a cholesterol lowering agent. To be submitted.

Manuscript 1: the candidate's role in this study was sharing responsibilities in running the animal trial collecting and analyzing data. The candidate wrote the manuscript with the guidance and feedback from co-authors.

Manuscript 2: the candidate was involved in running the animal trial, collecting data as well as reviewing and providing feedback for the manuscript.

Manuscript 3: the candidate was involved in writing the manuscript.

I, Christopher Marinangeli, co-author of the aforementioned manuscripts, hereby agree to their inclusion in the $\mathrm{PhD}$ thesis of the candidate, Amira Kassis.

$$
\text { (P) Minangeh. }
$$

Christopher Marinangeli 


\section{Manuscript inclusion in doctoral thesis waiver form \\ Permission from co-author after publication}

I hereby grant Amira Kassis permission to include the Manuscript entitled "Comparison of composition and absorption of sugarcane policosanols" which has been published in the British journal of Nutrition, in her Doctoral thesis.

$$
\text { (N) pumangeh }
$$

Christopher Marinangeli

$$
\text { Sept 1\%,2007 }
$$

Date 


\title{
Comparison of composition and absorption of sugarcane policosanols
}

\author{
Christopher P. F. Marinangeli ${ }^{1}$, Amira N. Kassis ${ }^{1}$, Deepak Jain ${ }^{1}$, Naoyuki Ebine ${ }^{1}$, Stephen C. Cunnane ${ }^{2}$ \\ and Peter J. H. Jones ${ }^{1,3 *}$ \\ ${ }^{1}$ School of Dietetics and Human Nutrition, McGill University, Ste. Anne de Bellevue, Québec, H9X 3V9 Canada \\ ${ }^{2}$ Research Centre on Aging, Université de Sherbrooke, Sherbrooke, Québec, J1H 4 C4 Canada \\ ${ }^{3}$ Richardson Centre for Functional Foods and Nutraceuticals, 196 Innovation Drive, University of Manitoba, \\ Winnipeg, Manitoba, R3T 6 C5 Canada \\ (Received 30 May 2006 - Revised 2 October 2006 - Accepted 6 October 2006)
}

Policosanols (PC) exist as very-long-chain alcohols derived from sugarcane currently used in many countries as a cholesterol-lowering therapy. PC purity and relative percentage composition have been suggested as primary reasons why the original Cuban PC (OPC) supplements possess lipidlowering efficacy. The purpose of the present study was, first, to compare the relative percentage purity and PC composition of both OPC and alternative sources of PC (APC). A second objective was to feed Syrian hamsters a diet containing $0 \cdot 275 \mathrm{mg}$ PC/g of either the OPC or an APC product (APC1) and compare subsequent tissue, plasma and faecal PC levels. Five animals from the APC1 dietary group received a diet containing ten times the original amount of PC. Results indicate that the APC formulations have a composition that is highly consistent with the OPC supplement, with octacosanol being present within the cited $60-70 \%$ range. PC were undetectable in the small intestine, liver, adipose or plasma in animals fed either source. Hamsters fed OPC excreted octacosanol $(\mathrm{C} 28)$ more rapidly $(P<0 \cdot 05)$ than hamsters receiving APC1. If the cholesterol-lowering efficacy of PC mixtures is dependent on their purity and composition, then sugarcane-derived APC products should possess similar therapeutic properties as the OPC supplement.

Policosanols: Cholesterol: Sugarcane: Composition: Absorption

Policosanols (PC) are primary aliphatic 24- to 34-carbon alcohols derived from the wax constituent of plants. Researchers in Cuba isolated PC from sugarcane (Saccharum officinarum L.) wax and developed the original PC supplement (OPC) (Gouni-Berthold \& Berthold, 2002; Anonymous, 2004), which was approved in 1991 in Cuba as a lipid-lowering therapy (Gouni-Berthold \& Berthold, 2002). Since then, the OPC supplement has been the subject of numerous human clinical investigations. Daily doses ranging from 2 to $20 \mathrm{mg}$ have been shown to be efficacious in safely producing dose-dependent reductions in total cholesterol (TC) and LDL-cholesterol (LDL-C) ranging from 10 to $23 \%$ and 11 to $31 \%$, respectively (Mas, 2000; GouniBerthold \& Berthold, 2002). Studies also show that PC are at least equally effective in lowering both TC and LDL-C as are prescription medications (Ortensi et al. 1997; Pons et al. 1997; Alcocer et al. 1999; Crespo et al. 1999; Castano et al. 2000, 2003). Animal and cell-culture studies have demonstrated that $\mathrm{PC}$ reduce cholesterol synthesis either by interfering with the production, degradation or activity of 3-hydroxy-3-methylglutaryl-CoA (HMG-CoA) reductase (Menendez et al. 1996 , $2001 a$; Singh et al. 2006), as well as by increasing cellular LDL uptake (Menendez et al. 1997).

Despite the impressive initial results for $\mathrm{PC}$, for the most part, research groups outside of Cuba using altemative sources of PC (APC) derived from either sugarcane or non-sugarcane sources have been unable to reproduce the initial reports in animals (Wang et al. 2003, 2005; Murphy et al. 2004) or in human subjects (Lin et al. 2004; Reiner et al. 2005; Berthold et al. 2006; Greyling et al. 2006). The relative composition of the OPC supplement has been published indicating a purity of $\geq 90 \%$, with octacosanol (C28) being the most abundant PC $(60-70 \%)$, followed by triacontanol $(\mathrm{C} 30 ; 10-15 \%)$, hexacosanol $(\mathrm{C} 26 ; 4 \cdot 5-10 \%)$, dotriacontanol $(\mathrm{C} 32 ; 3-8 \%)$, heptacosanol $(\mathrm{C} 27 ; \leq 5 \%)$, tetracosanol $(\mathrm{C} 24 ; \leq 2 \%)$, nonacosanol $(\mathrm{C} 29 ; \leq 2 \%)$ and tetratriacontanol $(\mathrm{C} 34 ; \leq 2 \%)$ (Mas, 2000). It is thought that since octacosanol is present in the highest quantity, it is the primary PC responsible for lowering cholesterol levels (Mas, 2000). The original researchers have defended their results by indicating that it is the exclusive purity and relative composition of the OPC supplement that are responsible for its ability to reduce blood lipids (Castano et al. 2002). A systematic comparison of OPC $v$. APC products has not, however, been undertaken.

PC metabolism has also been the subject of controversy. Due to the structure of $\mathrm{PC}$, their hydrophobicity appears to result in poor absorption (Hargrove et al. 2004), confirmed in both rats (Kabir \& Kimura, 1995; Menendez et al. 2005) and monkeys (Menendez et al. 2005) using radiolabelled $\left[8-{ }^{14} \mathrm{C}\right]$ octacosanol 
(Kabir \& Kimura, 1995) as well as in an octacosanol metabolism study using PC supplements (Menendez et al. 2005).

The purpose of the present study, therefore, was to first analyse various brands of the OPC and sugarcane-derived APC supplements and compare our analyses with the published data for the OPC product. A second objective was to evaluate and compare the plasma, tissue and faecal levels of octacosanol within animals fed either OPC or an APC product.

\section{Materials and methods}

\section{Compositional analysis}

Original and alternative sugarcane policosanol products. OPC tablets indicated as containing either 6 or $20 \mathrm{mg} \mathrm{PC} \mathrm{pro-}$ duced by Dalmer Laboratories were obtained from pharmacies in Cuba (OPC1 and $\mathrm{OPC} 2$ ) and Argentina (OPC3). OPC supplements were also obtained in Canadian natural health stores (OPC4 and OPC5). Sugarcane-derived APC products were provided as powders: APC1 (Degussa Bioactives, Champaign, IL, USA), APC2 (Lesstanol ${ }^{\circledR}$; Garuda International Inc., Lemon Cove, CA, USA) and APC3 (AHD International, LLC, Atlanta, GA, USA).

Policosanol tablet and powder analysis. Individual tablets were weighed, crushed and placed in a test-tube with chloroform. The powder-chloroform mixture was heated for $15 \mathrm{~min}$ at $60^{\circ} \mathrm{C}$ and filtered into another test-tube. The solution was dried under $\mathrm{N}_{2}$ gas and either $6 \mathrm{ml}$ (for $6 \mathrm{mg}$ tablets) or $10 \mathrm{ml}$ (for $20 \mathrm{mg}$ tablets) of chloroform was added to the dried sample and heated for $15 \mathrm{~min}$ at $60^{\circ} \mathrm{C}$. Samples of PC were transferred to another test-tube. The solution was dried under $\mathrm{N}_{2}$ and $200 \mu \mathrm{l}$ methylene chloride was added and heated for $15 \mathrm{~min}$ at $60^{\circ} \mathrm{C}$. The APC mixtures were prepared using the same procedure, except a $20 \mathrm{mg}$ sample was used.

Separation of PC was performed by GC using an Agilent 6890 GC (Mississauga, Canada) equipped with a flame ionisation detector and a fused capillary column (HP-1; $30 \mathrm{~m} \times 0.25 \mathrm{~mm}$ internal diameter, $0.25 \mu \mathrm{m}$ film thickness). The injector temperature was $330^{\circ} \mathrm{C}$ with a $50: 1$ split. The initial oven temperature was $150^{\circ} \mathrm{C}$ for $2 \mathrm{~min}$, then increased at $20^{\circ} \mathrm{C} / \mathrm{min}$ to $250^{\circ} \mathrm{C}$, then to $325^{\circ} \mathrm{C}$ at $6^{\circ} \mathrm{C} / \mathrm{min}$. The carrier gas was He at $1.0 \mathrm{ml} / \mathrm{min}$. Peaks were identified using standards for tetracosanol, hexacosanol, heptacosanol, octacosanol and triacontanol obtained from Sigma-Aldrich (Oakville, Canada). Nonacosanol and dotriacontanol were identified by extrapolation, while tetratriacontanol was identified using beeswax purchased from Sigma-Aldrich, plus information provided by Jimenez et al. (2003), and a certificate of analysis provided by Garuda Intemational Inc. PC compositions were compared with those reported by Mas (2000) outlining the purity and composition of the OPC supplement manufactured by Dalmer Laboratories (Havana, Cuba).

\section{Animal trial}

Animals and diets. This experiment was approved by the McGill Animal Care and Research Ethics committee in accordance with guidelines of the Canadian Council on Animal Care. The Syrian hamster was selected because it has been considered one of the best animal models for examining cholesterol metabolism (Kris-Etherton \& Dietschy, 1997).
Thirty-six Golden Syrian hamsters weighing $80-100 \mathrm{~g}$ were housed in individual cages. Animals were acclimatised to a $12 \mathrm{~h}$ light-dark cycle and fed regular rodent chow for a period of 2 weeks. All animals had free access to water for the entire duration of the investigation. Hamsters were randomly assigned to one of three experimental diets prepared according to the AIN-93G formula. Study diet 1 was a control diet consisting of casein $(20 \%)$, maize starch $(26 \%)$, sucrose $(33 \%)$, beef tallow-safflower-seed oil mix $(10 \%)$, cellulose $(5 \%)$, DL-methionine $(0.5 \%)$, mineral mixture $(4 \%)$, vitamin mixture $(1 \%)$, choline bitartrate $(0.2 \%)$, butylated hydroxytoluene $(0.002 \%)$ and cholesterol $(0.1 \%)$. Dietary groups 2 and 3 received the same dietary formulation but with the addition of sugarcane $\mathrm{PC}$ at $0.275 \mathrm{mg} / \mathrm{g}$ diet. Dietary group 2 (APC1) was given sugarcane PC provided by Degussa Bioactives, while dietary group 3 (OPC) was given sugarcane PC manufactured by Dalmer Laboratories. OPC was obtained in tablet form, crushed into a fine powder, and subsequently added to the diet. Five animals from the APC1 group were given a diet containing ten times the original amount $(2.75 \mathrm{mg} \mathrm{PC} / \mathrm{g}$ diet $)$ in order to observe whether elevated PC intake results in a proportional increase in plasma and tissue levels of PC. All animals were given free access to the experimental diets for the duration of the study. Food intake and food waste were recorded every $3 \mathrm{~d}$. Body weights were recorded every $7 \mathrm{~d}$.

After $28 \mathrm{~d}$ on their experimental diets, animals were anaesthetised by $\mathrm{CO}_{2}$ inhalation. Blood was collected via decapitation and plasma was separated from erythrocytes. The perirenal adipose tissue, liver and small intestine were immediately collected. Plasma and all tissues were stored at $-80^{\circ} \mathrm{C}$. Faeces were collected for each animal over the final $3 \mathrm{~d}$, immediately dried and stored at $-20^{\circ} \mathrm{C}$. On these $3 \mathrm{~d}$, leftover food and food waste was recorded daily to determine the daily intake.

Tissue and plasma analysis. Samples of liver, adipose and small intestine were homogenised in saline and extracted using chloroform-methanol (2:1, v/v) (Folch et al. 1957). Plasma was analysed using the same method. The samples were heated and shaken for $10 \mathrm{~min}$ at $60^{\circ} \mathrm{C}$ and then centrifuged at $1500 \mathrm{rpm}$ at $25^{\circ} \mathrm{C}$ for $5 \mathrm{~min}$ to maintain solubility of the PC. This extraction procedure was repeated three times. Extracts were dried under $\mathrm{N}_{2}$ and saponified using methanolic $\mathrm{KOH}$ at $90^{\circ} \mathrm{C}$ for $2 \mathrm{~h}$ with concurrent shaking. Total lipids were then extracted with three washes of petroleum ether. After each addition of petroleum ether, the saponified solution was simultaneously shaken and heated at $60^{\circ} \mathrm{C}$ and then centrifuged for $5 \mathrm{~min}$ at $25^{\circ} \mathrm{C}$. Extracts were then dried under $\mathrm{N}_{2}$. Methylene chloride was added to the tissue extracts for GC analysis. 5 $\alpha$-Cholestane (Sigma-Aldrich) was added as an internal standard for the quantification of PC.

Faecal analysis. Samples of faeces from each of the $3 \mathrm{~d}$ were ground into a fine powder. Faecal PC were extracted and quantified by the same method as outlined earlier. Data were interpreted by dividing the $3 \mathrm{~d}$ average amount of octacosanol excreted by the $3 \mathrm{~d}$ average amount of octacosanol consumed.

Gas chromatography analysis. Before samples were analysed the detection limits for PC of our GC were determined. Standards of tetracosanol, hexacosanol, heptacosanol, octacosanol and triacontanol were purchased from Sigma-Aldrich. Each standard was mixed with chloroform, making individual stock solutions of $1 \mathrm{mg} / \mathrm{ml}$ with $1 \mathrm{ml}$ samples of each solution being isolated. Serial dilutions using chloroform as the solvent 
produced final concentrations that were $1 / 4,1 / 16,1 / 64,1 / 256$, $1 / 1024,1 / 1536$ and $1 / 4096$ of the original stock solution. Samples of $1 \mathrm{ml}$ of each dilution were isolated, and $50 \mu \mathrm{l}$ of a $1 \mathrm{mg} / \mathrm{ml}$ stock solution of $5 \alpha$-cholestane was added to each test-tube as an internal standard. Samples were dried under $\mathrm{N}_{2}$ and re-dissolved in methylene chloride with sonication.

Preceding the analysis of sets of serial dilutions, a mixed standard containing $5 \alpha$-cholestane $(0.12 \mathrm{mg} / \mathrm{ml})$, tetracosanol $(0.12 \mathrm{mg} / \mathrm{ml})$, hexacosanol $(0.12 \mathrm{mg} / \mathrm{ml})$, octacosanol $(0.12 \mathrm{mg} /$ $\mathrm{ml})$ and triacontanol $(0.12 \mathrm{mg} / \mathrm{ml})$ was analysed. Results from these chromatograms were used for quantifying PC found in samples evaluated that same day.

Separation was achieved using a Hewlett Packard 5890 GC equipped with a flame ionisation detector and fused capillary column (SAC-5; $30 \mathrm{~m} \times 0.25 \mathrm{~mm}$ internal diameter, 0.25 $\mu \mathrm{m}$ film thickness) from Sigma-Aldrich. The injector temperature was $300^{\circ} \mathrm{C}$ and initial oven temperature was $160^{\circ} \mathrm{C}$. The oven temperature was increased to $245^{\circ} \mathrm{C}$ at $15^{\circ} \mathrm{C} / \mathrm{min}$ and held for $4 \mathrm{~min}$. The temperature was again increased to $280^{\circ} \mathrm{C}$ at $15^{\circ} \mathrm{C}$ min. After $4 \mathrm{~min}$, a final temperature of $305^{\circ} \mathrm{C}$ was reached at a rate of $40^{\circ} \mathrm{C} / \mathrm{min}$ and held for $11 \mathrm{~min}$. The carrier gas used was He. Samples were quantified using the internal standard method (Scott \& Perry, 1998). Since serial dilutions were used, the calculated concentrations for each dilution were log-transformed when presented.

Tissue, plasma and faecal samples were analysed using the same GC protocol. On each day of analysis the same mixed standard as mentioned earlier was analysed for the quantification of octacosanol.

\section{Statistical analysis}

All statistical analyses were completed using SAS version 8 (SAS Institute Inc., Cary, NC, USA). The PC content in all tissues and plasma was compared across the three treatment groups using one-way ANOVA. Significant differences between groups were assessed using the Tukey $a$ priori test. For the faecal analysis, the average octacosanol intake as well as the average octacosanol excretion was determined over the $3 \mathrm{~d}$ in which faeces was collected. The ratio between the average amount $(\mathrm{mg})$ of octacosanol excreted $v$, the average amount (mg) of octacosanol ingested was computed for each animal. Data were then subjected to the arcsine transformation to achieve normality. The resultant data were then analysed by ANOVA and significant differences between groups were determined using the Tukey test. For reporting purposes, the arcsine-transformed ratio estimates for each PC group were changed back to their original values. However, the reported $P$ value was taken from the transformed analysis. For all statistical analysis, data are presented as mean values with their standard errors; significance was established at $P<0.05$.

\section{Results}

\section{Compositional analysis}

The results comparing various OPC supplements to the cited OPC purity and composition are summarised in Table 1. All of the OPC supplements met the specified requirements for the major $\mathrm{PC}$ constituents and the detectable minor

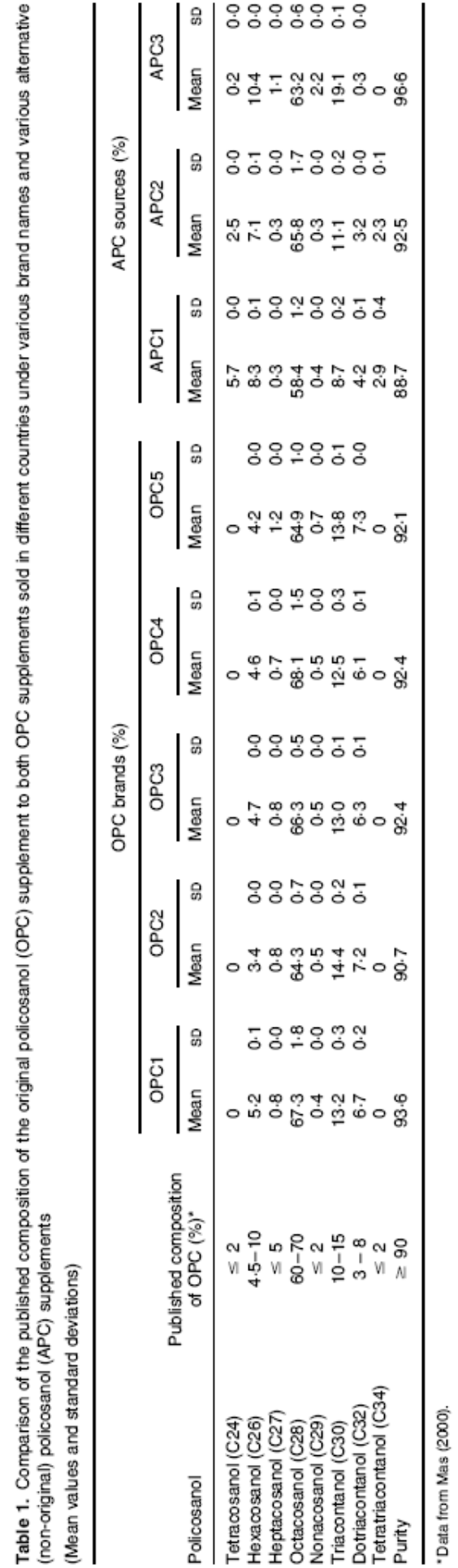


constituents. One exception was with OPC2, which had a percentage composition of hexacosanol that was slightly lower than the cited published range of $4 \cdot 5-10 \%$ (Mas, 2000). None of the OPC supplements contained detectable levels of tetracosanol and tetratriacontanol.

When APC were compared with the published composition of the original mixture, APC2 and APC 3 had purities greater than the $90 \%$, while the APC1 formulation had a slightly lower value at $88.7 \%$ (Table 1). Octacosanol, the major component thought to be responsible for cholesterol-lowering efficacy, was present within the $60-70 \%$ range for both $\mathrm{APC} 2$ and $\mathrm{APC} 3$, while the amount found in APC1 was only $1.6 \%$ below the cited $60 \%$ limit. Additionally, a notable departure from the original published range for triacontanol was observed in APC3.

\section{Animal trial}

Animal tissue, plasma and faecal analysis. The GC detection limits for pure tetracosanol, hexacosanol, heptacosanol, octacosanol and triacontanol were identified as 651.6, 431.8, $377.8,402 \cdot 1$ and $430 \cdot 2 \mathrm{ng} / \mathrm{ml}$, respectively.

No differences in body weight or food intake were observed among animals for the duration of the study. PC were not detectable in tissues or plasma in any of the animals receiving either OPC or APC1 mixtures, even at an intake of $2.75 \mathrm{mg} / \mathrm{g}$ diet. Large amounts of ingested octacosanol were recovered within the faeces. Compared with the amount of PC ingested, the total amount of octacosanol recovered for animals consuming either OPC or APC1 ranged from 24.7 to $89.7 \%$ (Fig. 1). For animals consuming the diet containing 10-fold the amount of APC1 of the other groups, 43-9-51.7\% of ingested octacosanol was recovered. Percentage excretion of octacosanol was $43 \%$ higher $(P=0.01)$ in the animals consuming OPC (55.8 (SEM 0.29)\% compared with APC1 $(38.8$
(SEM 0-45) \%) (Fig. 1). The excretion of octacosanol was $37 \%$ lower in the animals receiving higher levels of APC1 compared with animals receiving OPC, coupled with a strong trend towards significance $(P=0 \cdot 05)$. There was no difference $(P=0-83)$ in the amount of octacosanol excreted relative to the amount ingested between animals receiving the normal and higher levels of APC1.

\section{Discussion}

Regardless of the brand, the sugarcane-derived PC analysed in the present study demonstrated a similar PC purity and composition. For the OPC supplements, a similar composition between brands indicates consistent manufacturing practices regardless of where the product is sold. Nonetheless, contrary to the cited analysis of the OPC product, neither tetracosanol nor tetratriacontanol were detectable in any of the OPC (Table 1). The cited compositional analyses of the OPC supplement classify tetracosanol and tetratriacontanol as minor constituents comprising of $<2 \%$ of the total PC mixture (Mas, 2000). Consequently, their importance in contributing to the cholesterol-lowering efficacy of the OPC supplement is unknown. Analysis of APC1 showed an octacosanol composition and $\mathrm{PC}$ purity that was $<2 \%$ below the cited limits of the OPC product (Table 1). Whether this small reduction in octacosanol would substantially affect cholesterol-lowering efficacy seems unlikely.

One of the more important observations of the present study concerns the composition of APC2. The APC2 formulation is Lesstanol $^{\star}$, the same sugarcane-derived PC mixture previously compared with the OPC supplement by the origina Cuban research group. Compared with OPC, Lesstanol ${ }^{\text {ब }}$ failed to reduce TC and LDL-C levels to the same extent as OPC (Castano et al. 2002). The original researchers reported

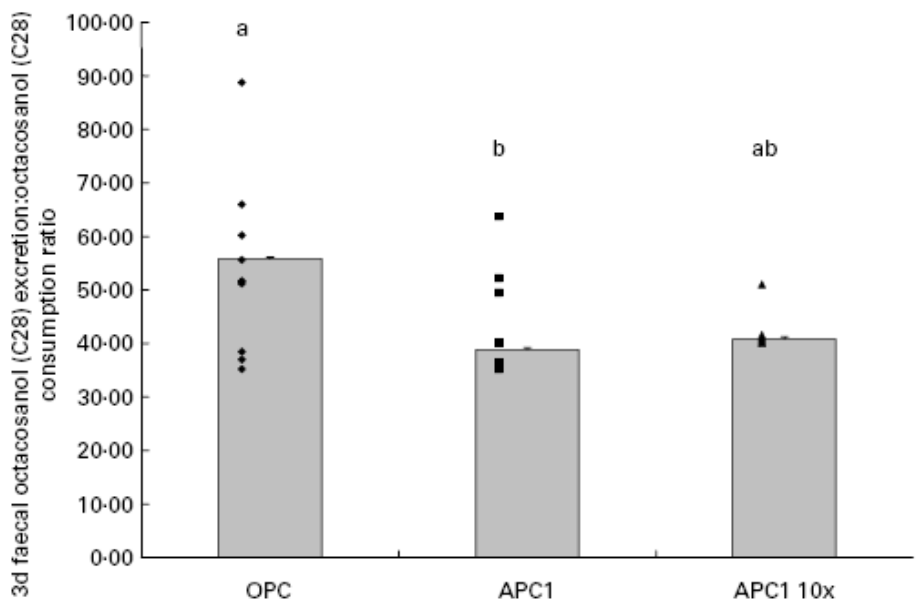

Fig. 1. Comparison of the average $3 \mathrm{~d}$ faecal octacosanol excretion:octacosanol consumption ratio. Statistics were performed using arcsine transformation. The reported data have been back-transformed revealing the values obtained before the arcsine was applied. OPC, orignal policosanol (PC) ( $\bullet)$; APC1, altemative

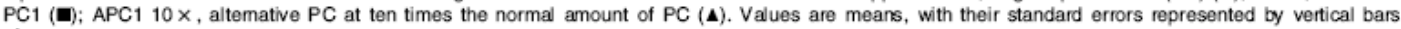
a.b Mean values with unlike letters were significantly different (data in transformed state) $(P<0.05)$. 
that Lesstanol ${ }^{\mathbb{1}}$ was less effective in reducing TC and LDL-C since compared with the OPC formulation, this APC mixture was shown to have a lower purity as well as a different PC composition, including notably lower levels of octacosanol (Castano et al. 2002). Results from the present study indicate that the purity and relative percentage composition of the PC contained in APC2 are actually comparable with the OPC product. If PC efficacy is dependent on the relative percentage composition of the PC formulation, then Lesstanol ${ }^{\circledR}$ would be expected to be as effective as the OPC supplement in lowering blood TC and LDL-C levels.

It has been suggested that PC could possibly affect the membrane fluidity of organelles responsible for manufacturing endogenous cholesterol, resulting in reduced synthesis or increased degradation of cellular HMG-CoA reductase (Menendez et al. 2001a). This theory stems from studies which outline that changes in the cellular and/or organelle membrane fatty acid composition can manipulate membrane fluidity and specifically affect HMG-CoA reductase activity (Davis \& Poznansky, 1987; Whitcomb et al. 1988; Garcia-Pelayo et al. 2003). Perhaps the relative composition of PC mixtures can produce specific membrane fluidity changes in organelles responsible for endogenous cholesterol synthesis; more specifically, that the published compositional range concerning the OPC formulation may produce an exclusive membrane fluidity that would have the greatest effect on reducing HMG-CoA reductase activity. Another theory attempting to explain the action of PC on HMG-CoA reductase suggests that triacontanol activates AMP-kinase. An in vitro study by Singh et al. (2006) found that the addition of PC to cultured hepatoma cells significantly inhibited cholesterol synthesis. Thus far, octacosanol was thought to be the active component of the OPC supplement responsible for its cholesterol-lowering properties. When the individual PC were tested, only triacontanol had an inhibitory effect. An increase in AMP-kinase phosphorylation was also noted (Singh et al. 2006). The researchers suggest that since phosphorylated AMP-kinase is a known inhibitor of HMGCoA reductase, the activation of AMP-kinase by triacontanol found in PC supplements could be the mechanism behind PC cholesterol-lowering efficacy (Singh et al. 2006). Nonetheless, human PC supplementation studies conducted by external research groups using either OPC or APC products have failed to show cholesterol-lowering efficacy. In a recent human clinical trial, $20 \mathrm{mg} / \mathrm{d}$ of the APC product Lesstanol ${ }^{\circledR \Phi}$ was administered to hypercholesterolaemic patients. After 12 weeks of treatment, Lesstanol ${ }^{\circledR}$ failed to produce any changes in serum lipid levels (Greyling et al. 2006). However, a recent study by Berthold et al. (2006), was the first external research group to use OPC in hypercholesterolaemic and hyperlipidaemic patients. OPC was administered at $10,20,40$ and $80 \mathrm{mg} / \mathrm{d}$ to four different treatment groups over a 12 -week period. Similar to the results reported by Greyling et al. (2006), OPC was unable to elicit significant reductions in TC and LDL-C compared with placebo. Nonetheless, the OPC used in the Berthold et al. (2006) study was purchased as a raw material, then subsequently manufactured into tablets. To our knowledge, external research centres have yet to publish data testing PC efficacy in a clinical setting using the OPC product administered as supplemental tablets produced in Cuba.

Initially, dietary and supplemental PC are thought be transported to the liver via chylomicrons (Hargrove et al. 2004).
Next, they enter the endoplasmic reticulum and are metabolised to their corresponding acid via the fatty alcohol cycle using the enzyme alcohol:NAD ${ }^{+}$oxidoreductase (Lee, 1979; Rizzo et al. 1987; Hargrove et al. 2004). Research indicates that the mitochondria are unable to metabolise very-longchain fatty acids (Jakobs \& Wanders, 1991; Mannaerts et al. 2000; Olivier \& Krisans, 2000; Wanders et al. 2000, 2001). It has been shown that these molecules are partially metabolised or 'chain shortened' by substrate-specific $\beta$-oxidative enzymes contained in the peroxisome. The resulting acids can then be transported to the mitochondria for further processing (Jakobs \& Wanders, 1991; Mannaerts et al. 2000; Olivier \& Krisans, 2000; Wanders et al. 2000, 2001). This sequence of events is ideal for the proposed mechanism of action outlined. It has been suggested that the PC corresponding acids are responsible for the observed therapeutic effect on lipid levels (Mendoza et al. 2001; Menendez et al. 2001b; Gamez et al. 2003). Thus, it is possible that some of the very-long-chain fatty acids produced from PC supplements are directly incorporated into the peroxisome membrane and perhaps affecting HMG-CoA reductase activity. The notion that $\mathrm{PC}$ lower cholesterol through an effect on peroxisomes would be consistent with the fact that the peroxisome is the major site for endogenous cholesterol biosynthesis and contains the highest levels of HMG-CoA reductase (Hodge et al. 1991; Mandel et al. 1995; Olivier \& Krisans, 2000). However, for this to occur, adequate amounts of supplemental PC must be absorbed.

In the present animal trial, our data indicate that supplemental PC were undetectable in animals receiving either $\mathrm{OPC}$ or the APCl formulation as well as animals receiving the diet containing ten times the original amount of APC1. Furthermore, no changes in serum lipid levels were noted. The original researchers have demonstrated cholesterol-lowering efficacy of PC using a genetically diverse range of models including human subjects (Aneiros et al. 1995), monkeys (Rodriguez-Echenique et al. 1994), rabbits (Arruzazabala et al. 1994; Menendez et al. 1997), dogs (Mesa et al. 1994) and rats (Menendez et al. 1996). Hence, it is unlikely that our observations are due to the hamster model utilised in the present study. The hypothesis outlined earlier regarding PC composition and its mechanism for lowering HMG-CoA reductase activity in the liver would require that adequate amounts of the supplement is absorbed and hence would be detected in both the plasma and hepatocytes. Studies indicate that as both the chain length (Sallee \& Dietschy, 1973; Sallee, 1979; Bernard \& Carlier, 1991) and degree of saturation (Jones et al. $1985 a, b)$ increase, the intestinal absomtion of fatty acids is significantly reduced. Given that PC have distinctly long carbon backbones and are entirely saturated may explain the observations noted in the present study.

Previous research has been able to detect supplementary octacosanol in tissues and/or plasma using radioisotopes (Kabir \& Kimura, 1993, 1995) or GC-MS (Menendez et al. 2005). It has been previously suggested that when using radioactivity as a means of quantifying tissue octacosanol, the analysis cannot discriminate between the parent compound and possible metabolites (Lin et al. 2004). Thus, it cannot be discounted that compound degradation could have occurred in the small intestine before absorption or in the liver 
post-absorption. The minimum GC detection limits of PC were assessed in the present study using serial dilutions of pure PC standards dissolved in chloroform. This method produced chromatograms that contained no additional compounds that could interfere with the detection and quantification of the exceedingly low levels of PC. However, during the analysis of tissue and plasma, it cannot be excluded that tetracosanol, hexacosanol, heptacosanol and triacontanol may have eluted from the GC at identical retention times as were other substances, impeding the identification of the PC of interest. Standard solutions indicated that the retention time for octacosanol was close to that of cholesterol, which could impede the quantification of octacosanol at very low concentrations. However, a study by Menendez et al. (2005) utilised GC-MS to detect plasma levels of octacosanol from a $10 \mathrm{mg} / \mathrm{kg}$ oral dose of PC supplement in monkeys. Plasma levels of octacosanol peaked at over $400 \mathrm{ng} / \mathrm{ml}$ at $1 \mathrm{~h}$ after ingestion. In the same study when rats were given $60 \mathrm{mg} \mathrm{PC} / \mathrm{kg}$ via gastric tube feeding, the maximum concentration of octacosanol found in the plasma and tissues was $30.4 \mathrm{ng} / \mathrm{ml}$ and $68.4 \mathrm{ng} / \mathrm{g}$ respectively. Relative to the dose of PC administered, the amount discerned is small, reinforcing the notion of the poor absorption of PC. In the Menendez et al. (2005) study, the maximum plasma levels of octacosanol observed in rats were well below the $402.1 \mathrm{ng} / \mathrm{ml}$ detection limit for octacosanol of the present study. GC-MS is a more sensitive means of detection than GC methods. Considering the maximum levels of tissue and plasma octacosanol observed by Menendez et al. (2005) post-PC supplementation, it is unlikely that the amount of PC consumed in the present study would have produced plasma and tissue octacosanol concentrations greater than the $402.1 \mathrm{ng} / \mathrm{ml}$ detection limit observed for our GC.

In the present study, hamsters consumed approximately $2 \mathrm{mg}$ PC supplement per d. Based on the animals' initial body weight and average food intake, this amount was equivalent to approximately $18 \mathrm{mg} / \mathrm{kg}$ per $\mathrm{d}$. Animals receiving the APC1-fortified diet at ten times the normal amount of PC, corresponded to approximately $183 \mathrm{mg} / \mathrm{kg}$ per $\mathrm{d}$. In human patients the maximum therapeutic dose of $\mathrm{PC}$ is $20 \mathrm{mg} / \mathrm{d}$ (Castano et al. 2001). In a $70 \mathrm{~kg}$ individual this amount is equivalent to $0 \cdot 285 \mathrm{mg} / \mathrm{kg}$. Based on these data, significant alterations in cholesterol metabolism are hard to explain when the low absorption of PC is considered. Further metabolic studies conducted on human subjects, using reasonable therapeutic doses of $\mathrm{PC}$ and perhaps stable isotopes, are required to conclusively define the mechanism concerning the cholesterol-lowering effects of PC.

A greater data range referring to octacosanol recovery was noted between animals receiving $0.275 \mathrm{mg} \mathrm{PC/g}$ diet (OPC and APC1) compared with animals receiving $2.75 \mathrm{mg} \mathrm{PC} / \mathrm{g}$ diet at 24.7 to $89.7 \%$ and 43.1 to $51.7 \%$, respectively. This observation could be due to fewer animals receiving the higher dose of $\mathrm{PC}$ in the diet, resulting in a comparatively smaller number of outliers. It is unknown what happened to the remainder of the ingested octacosanol. The present results show that PC were not present in the tissues or plasma of animals receiving $\mathrm{PC}$ treatment. It can be assumed that some $\mathrm{PC}$ residuals would have remained within the digestive tracts of the animals at the time of euthanasia. The amount of residual octacosanol left within the gastrointestinal tract could have been affected by the PC treatment. Studies have shown that as fatty acid chain length increases, the release of cholecystokinin after ingestion decreases (McLaughlin et al. 1999; Jonkers et al. 2000). Cholecystokinin is one of the hormones secreted postprandially which facilitates gastric motility (Whitney \& Rolfes, 2002). Since PC have distinctly long carbon backbones and are structurally similar to fatty acids, PC could reduce the postprandial secretion of certain hormones, including cholecystokinin, suppressing gastrointestinal motility. Finally, bacterial degradation of PC could have also affected the amount of octacosanol recovered in the faeces. Although we were unable to find studies alluding to colonic fermentation of $\mathrm{PC}$ and very-long-chain acids, microbial breakdown is a metabolic fate of PC that remains to be delineated.

Analysis regarding PC excretion indicated that animals consuming the diet containing OPC had significantly higher levels of octacosanol in the faeces in relation to those consuming the APC1 formulation (Fig. 1). Furthermore, a strong trend towards greater octacosanol excretion in the OPC1 group compared with animals receiving ten times the normal amount of APC1 was also observed. Whether the differences observed between APC1 and OPC regarding octacosanol excretion can be explained by the slight difference in PC composition is yet to be determined. The observation that octacosanol excretion was similar between groups receiving normal and high levels of APC1 reinforces the notion that either OPC or APC1 instigates a physiological effect. In theory, equivalent intakes would provide APC1 with a longer resident time for absorption, increasing the opportunity for detection. Researchers recently observed that PC significantly increased bile acid excretion in hamsters from 25 to $57 \%$ with diets containing 380,750 and $1500 \mathrm{mg} \mathrm{PC} / \mathrm{kg}$ diet, resulting in a significant reduction in $\mathrm{TC}$ by 15 to $25 \%$ ( $\mathrm{Ng}$ et al. 2005). Hence, the increased rate of excretion of OPC observed in the present study may have therapeutic significance in connection with reducing levels of circulating cholesterol. However, the PC described by $\mathrm{Ng}$ et al. (2005) were obtained as pure octacosanol, triacontanol and hexacosanol. Therefore, these results cannot be completely extrapolated to answer questions concerning differences observed with respect to cholesterol-lowering efficacy between PC supplements. Nonetheless, the relationship between PC supplementation and bile acid excretion as a potential mechanism for decreasing circulating cholesterol should be further explored.

In summary, results from the present study indicate that the Cuban OPC supplements sold in various countries under different brand names have a purity and relative percentage PC composition that are consistent with the cited literature referring to the same product. Contrary to previous research, sugarcane-derived APC formulations have a purity and percentage composition similar to the OPC mixture. Thus, APC supplements should possess similar cholesterollowering properties if the previous conception concerning $\mathrm{PC}$ efficacy hinges on the importance of PC purity and composition. In the present study, $\mathrm{PC}$ were undetectable in the plasma and tissues of hamsters receiving diets fortified with either the OPC or a sugarcane-derived APC product. Moreover, octacosanol from the APC1 diet was excreted at slower rates. Further research comparing the metabolism of PC from the OPC and APC supplements using therapeutic doses will help determine if mechanistic differences do exist between products. 


\section{Acknowledgements}

The authors acknowledge Mary Anne Ryan for her technical assistance and The Advanced Foods and Materials Network for their financial support. We also thank Degussa Bioactives, Garuda International Inc. and AHD Intemational, LLC for providing altemative sugarcane PC products.

\section{References}

Alcocer L, Fernandez L, Campos E \& Mas R (1999) A comparative study of policosanol versus acipimox in patients with type II hypercholesterolemia. Int J Tissue React 21, 85-92.

Aneiros E. Mas R, Calderon B, Illnait J, Fernandez L, Castano G \& Fernandez JC (1995) Effect of policosanol in lowering cholesterol levels in patients with type II hypercholesterolemia. Curr Ther Res Clin Exp 56, 176-181.

Anonymous (2004) Monograph. Policosanol. Altern Med Rev 9. 312-317.

Arruzazabala ML, Carbajal D, Mas R, Molina V, Valdes S \& Laguna A (1994) Cholesterol-lowering effects of policosanol in rabbits. Biol Res 27, 205-208.

Bernard A \& Carlier H (1991) Absorption and intestinal catabolism of fatty acids in the rat: effect of chain length and unsaturation. Exp Physiol 76, 445-455.

Berthold HK, Unverdorben S, Degenhardt R, Bulitta M \& Gouni-Berthold I (2006) Effect of policosanol on lipid levels among patients with hypercholesterolemia or combined hyperlipidemia: a randomized controlled trial. JAMA 295, 2262-2269.

Castano G, Fernandez L, Mas R. Illnait J, Fernandez J, Mesa M, Alvarez E \& Lezcay M (2002) Comparison of the efficacy, safety and tolerability of original policosanol versus other mixtures of higher aliphatic primary alcohols in patients with type II hypercholesterolemia. Int J Clin Pharmacol Res 22, 55-66.

Castano G, Mas R, Fernandez J, Fernandez L, Alvarez E \& Lezcay M (2000) Efficacy and tolerability of policosanol compared with lovastatin in patients with type II hypercholesterolemia and concomitant coronary risk factors. Curr Ther Res Clin Exp 61, 137-146.

Castano G, Mas R, Fernandez L, Illnait J, Gamez R \& Alvarez E (2001) Effects of policosanol 20 versus $40 \mathrm{mg} /$ day in the treatment of patients with type II hypercholesterolemia: a 6-month doubleblind study. Int J Clin Pharmacol Res 21, 43-57.

Castano G, Mas R, Fernandez L, Illnait J, Mesa M, Alvarez E \& Lezcay M (2003) Comparison of the efficacy and tolerability of policosanol with atorvastatin in elderly patients with type II hypercholesterolaemia. Drugs Aging 20, 153-163.

Crespo N, Illnait J, Mas R, Fernandez L, Fernandez J \& Castano G (1999) Comparative study of the efficacy and tolerability of policosanol and lovastatin in patients with hypercholesterolemia and noninsulin dependent diabetes mellitus. Int J Clin Pharmacol Res 19, $117-127$.

Davis PJ \& Poznansky MJ (1987) Modulation of 3-hydroxy3-methylglutaryl-CoA reductase by changes in microsomal cholesterol content or phospholipid composition. Proc Natl Acad Sci USA 84, 118-121.

Folch J, Lees M \& Sloane Stanley GH (1957) A simple method for the isolation and purification of total lipides from animal tissues. J Biol Chem 226, 497-509.

Gamez R, Mendoza S, Mas R, Noa M, Arruzazabala L, Carbajal D, Castano G, Goicochea E, Mesa M \& Mendoza N (2003) Comparison of the cholesterol-lowering effects and toxicity of D-003 and lovastatin in normocholesterolaemic rabbits. Drugs $R \quad D \quad 4$ 219-229.

Garcia-Pelayo MC, Garcia-Peregrin E \& Martinez-Cayuela M (2003) Modification of phospholipids fatty acid composition in reuber H35 hepatoma cells: effect on HMG-CoA reductase activity. J Cell Biochem 90, 586-591.

Gouni-Berthold I \& Berthold HK (2002) Policosanol: clinical pharmacology and therapeutic significance of a new lipid-lowering agent. Am Heart $J$ 143, 356-365.

Greyling A, De Witt C, Oosthuizen W \& Jerling JC (2006) Effects of a policosanol supplement on serum lipid concentrations in hypercholesterolaemic and heterozygous familial hypercholesterolaemic subjects. Br J Nutr 95, 968-975.

Hargrove JL, Greenspan P \& Hartle DK (2004) Nutritional significance and metabolism of very long chain fatty alcohols and acids from dietary waxes. Exp Biol Med (Maywood) 229, 215-226.

Hodge VJ, Gould SJ, Subramani S, Moser HW \& Krisans SK (1991) Normal cholesterol synthesis in human cells requires functional peroxisomes. Biochem Biophys Res Commun 181, 537-541.

Jakobs BS \& Wanders RJ (1991) Conclusive evidence that very-longchain fatty acids are oxidized exclusively in peroxisomes in human skin fibroblasts. Biochem Biophys Res Commun 178, 842-847.

Jimenez JJ, Bernal JL, Aumente S, Toribio L \& Bernal J Jr (2003) Quality assurance of commercial beeswax II. Gas chromatography-electron impact ionization mass spectrometry of alcohols and acids. J Chromatogr A 1007, 101-116.

Jones PJ, Pencharz PB \& Clandinin MT (1985a) Absorption of ${ }^{13} \mathrm{C}$-labeled stearic, oleic, and linoleic acids in humans: application to breath tests. J Lab Clin Med 105, 647-652.

Jones PJ, Pencharz PB \& Clandinin MT (1985b) Whole body oxidation of dietary fatty acids: implications for energy utilization. Am J Clin Nutr 42, 769-777.

Jonkers IJ, Ledeboer M, Steens J, Smelt AH \& Masclee AA (2000) Effects of very long chain versus long chain triglycerides on gastrointestinal motility and hormone release in humans. Dig Dis Sci 45, 1719-1726

Kabir Y \& Kimura S (1993) Biodistribution and metabolism of orally administered octacosanol in rats. Ann Nutr Metab 37, 33-38.

Kabir Y \& Kimura S (1995) Tissue distribution of (8-14C)-octacosanol in liver and muscle of rats after serial administration. Ann Nutr Metab 39, 279-284.

Kris-Etherton PM \& Dietschy J (1997) Design criteria for studies examining individual fatty acid effects on cardiovascular disease risk factors: human and animal studies. Am J Clin Nutr 65, 1590S-1596S.

Lee T (1979) Characterization of fatty alcohol: $\mathrm{NAD}^{+}$oxidoreductase from rat liver. J Biol Chem 254, 2892-2896.

Lin Y, Rudrum M, van der Wielen RP, Trautwein EA, McNeill G, Sierksma A \& Meijer GW (2004) Wheat germ policosanol failed to lower plasma cholesterol in subjects with normal to mildly elevated cholesterol concentrations. Metabolism 53, 1309-1314.

McLaughlin J, Grazia Luca M, Jones MN, D'Amato M. Dockray GJ \& Thompson DG (1999) Fatty acid chain length determines cholecystokinin secretion and effect on human gastric motility. Gastroenterology 116, 46-53.

Mandel H, Getsis M, Rosenblat M, Berant M \& Aviram M (1995) Reduced cellular cholesterol content in peroxisome-deficient fibroblasts is associated with impaired uptake of the patient's low density lipoprotein and with reduced cholesterol synthesis. J Lipid Res 36, 1385-1391.

Mannaerts GP, Van Veldhoven PP \& Casteels M (2000) Peroxisomal lipid degradation via $\beta$ - and $\alpha$-oxidation in mammals. Cell Biochem Biophys 32, 73-87.

Mas R (2000) Policosanol. Hypolipidemic, antioxidant, treatment of atherosclerosis. Drugs Future 25, 569-586.

Mendoza S, Gamez R, Noa M, Mas R, Castano G, Mesa AR, Mesa M \& de Armas M (2001) Comparison of the effects of D-003 and policosanol on lipid profile and endothelial cells in normocholesterolemic rabbits. Curr Ther Res Clin Exp 62, 209-220.

Menendez R, Amor AM, Gonzalez RM, Fraga V \& Mas R (1996) Effect of policosanol on the hepatic cholesterol biosynthesis of normocholesterolemic rats. Biol Res 29, 253-257. 
Menendez R, Amor AM, Rodeiro I, Gonzalez RM, Gonzalez PC, Alfonso JL \& Mas R (2001a) Policosanol modulates HMG$\mathrm{CoA}$ reductase activity in cultured fibroblasts. Arch Med Res 32, $8-12$.

Menendez R, Arruzazabala L, Mas R, et al. (1997) Cholesterol lowering effect of policosanol on rabbits with hypercholesterolaemia induced by a wheat starch-casein diet. $\mathrm{Br} J$ Nutr 77, 923-932.

Menendez R, Marrero D, Mas R, Fernandez I, Gonzalez L \& Gonzalez RM (2005) In vitro and in vivo study of octacosanol metabolism. Arch Med Res 36, 113-119.

Menendez R, Mas R, Amor AM, Rodeiros I, Gonzalez RM \& Alfonso JL (2001) Inhibition of cholesterol biosynthesis in cultured fibroblasts by D003, a mixture of very long chain saturated fatty acids. Pharmacol Res 44, 299-304.

Mesa AR, Mas R, Noa M, Hernandez C, Rodeiro I, Gamez R, Garcia M. Capote A \& Aleman CL (1994) Toxicity of policosanol in beagle dogs: one-year study. Toxicol Lett 73, 81-90.

Murphy KJ, Saint DA \& Howe PR (2004) Lack of effect of sugar cane and sunflower seed policosanols on plasma cholesterol in rabbits. Asia Pac J Clin Nutr 13, S69.

$\mathrm{Ng} \mathrm{CH}$, Leung KY, Huang Y \& Chen ZY (2005) Policosanol has no antioxidant activity in human low-density lipoprotein but increases excretion of bile acids in hamsters. J Agric Food Chem 53, $6289-6293$.

Olivier LM \& Krisans SK (2000) Peroxisomal protein targeting and identification of peroxisomal targeting signals in cholesterol biosynthetic enzymes. Biochim Biophys Acta 1529, 89-102.

Ortensi G, Gladstein J, Valli H \& Testone P (1997) A comparative study of policosanol versus simvastatin in elderly patients with hypercholesterolemia. Curr Ther Res Clin Exp 58, 390-301.

Pons P, Illnait J, Mas R, Rodriguez M, Aleman C, Fernandez JC, Fernandez L \& Martin M (1997) A comparative study of policosanol versus probucol in patients with hypercholesterolemia. Curr Ther Res Clin Exp 58, 26-35.

Reiner Z, Tedeschi-Reiner E \& Romic Z (2005) Effects of rice policosanol on serum lipoproteins, homocysteine, fibringogen and C-reactive protein in hypercholesterolaemic patients. Clin Drug Invest 25, 701-707.
Rizzo WB, Craft DA, Dammann AL \& Phillips MW (1987) Fatty alcohol metabolism in cultured human fibroblasts. Evidence for a fatty alcohol cycle. J Biol Chem 262, 17412-17419.

Rodriguez-Echenique C, Mesa R, Mas R, Noa M, Menendez R, Gonzalez RM, Amor AM, Fraga V, Sotolongo V \& Laguna A (1994) Effects of policosanol chronically administered in male monkeys (Macaca arctoides). Food Chem Toxicol 32, 565-575.

Sallee VL (1979) Permeation of long-chain fatty acids and alcohols in rat intestine. Am J Physiol 236, E721-E727.

Sallee VL \& Dietschy JM (1973) Determinants of intestinal mucosal uptake of short- and medium-chain fatty acids and alcohols. J Lipid Res 14, 475-484.

Scott RPW \& Perry JA (1998) Introduction to Analytical Gas Chromatography, 2nd ed. New York: Marcel Dekker.

Singh DK, Li L \& Porter TD (2006) Policosanol inhibits cholesterol synthesis in hepatoma cells by activation of AMP-kinase. $J$ Pharmacol Exp Ther 318, 1020-1026.

Wanders RJ, van Grunsven EG \& Jansen GA (2000) Lipid metabolism in peroxisomes: enzymology, functions and dysfunctions of the fatty acid $\alpha$ - and $\beta$-oxidation systems in humans. Biochem Soc Trans 28, 141-149.

Wanders RJ, Vreken P, Ferdinandusse S, Jansen GA, Waterham HR, van Roermund CW \& van Grunsven EG (2001) Peroxisomal fatty acid $\alpha$ - and $\beta$-oxidation in humans: enzymology, peroxisomal metabolite transporters and peroxisomal diseases. Biochem Soc Trans 29, 250-267.

Wang Y, Ebine N, Jia X, Jones PJ, Fairow C \& Jaeger R (2005) Very long chain fatty acids (policosanols) and phytosterols affect plasma lipid levels and cholesterol biosynthesis in hamsters. Metabolism 54, 508-514.

Wang YW, Jones PJ, Pischel I \& Fairow C (2003) Effects of policosanols and phytosterols on lipid levels and cholesterol biosynthesis in hamsters. Lipids 38, 165-170.

Whitcomb RW, Linehan WM \& Knazek RA (1988) Effects of longchain, saturated fatty acids on membrane microviscosity and adrenocorticotropin responsiveness of human adrenocortical cells in vitro. J Clin Invest $81,185-188$.

Whitney EN \& Rolfes SR (2002) Understanding Nutrition, chapter 3 pp. 64-91. Belmont, CA: Wadsworth. 


\section{Manuscript 3-Published}

Lack of of cholesterol-lowering efficacy of Cuban Sugar cane policosanols in hypercholesterolemic persons

Amira N. Kassis and Peter J.H. Jones

Published in the American Journal of Clinical Nutrition 
F-952

Subject: Authorization request

Dear Dr. Bier,

The manuscript entitled "Lack of cholcsterol-lowering efficacy of Cuban sugar cane policosanols in hypercholcsterolemic persons" published in the American Journal of Clinical Nutrition in 2006 is part of my $\mathrm{PhD}$ thesis project. As I am soon to submit my thesis, I was wondering what the procedure was to get an authorization from you to include this manuscript in my thesis.

The exact refercace is:

Lack of cholesterol-lowering efficacy of Cuban sugar cane policosanols in bypercholesterolemic persons. Am. J. Clinical Nutrition, Nov 2006; 84: $1003-1008$,

Sincerely,

Amira Kassis.

Permission granied by the copyright owner provided complete eredit is given to the original source: Am. J. Clin. Nutr. (year;volume:page range). American Socicty for Nutrition. As a courtesy, please notily the author of your intent to usc this praterial.

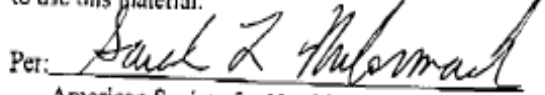

American Society for Nutrition 
September 17, 2007

To Dr. Peter Jones

The purpose of this letter is to confirm that Dr. Peter Jones, co-author of the below mentioned manuscripts, agrees to their inclusion in the thesis of Amira Kassis, $\mathrm{PhD}$ candidate.

\section{Manuscripts to be included}

1- Kassis AN, Marinangeli CP, Jain D, Ebine N \& Jones PJ (2006) Lack of effect of sugar cane policosanol on plasma cholesterol in golden Syrian hamsters.

Atherosclerosis. Epub ahead of print

2- Marinangeli CP, Kassis AN, Jain D, Ebine N, Cunnane SC \& Jones PJ (2007)

Comparison of composition and absorption of sugarcane policosanols. Br J Nutr. Feb;97(2):381-8

3- Kassis AN \& Jones PJ (2006) Lack of cholesterol-lowering efficacy of Cuban sugar cane policosanols in hypercholesterolemic persons. Am J Clin Nutr. 2006 Nov;84(5):10

4- Kassis AN \& Jones PJH. Changes in cholesterol metabolism in response to sugar cane policosanol in hypercholesterolemic individuals. To be submitted.

5- Kassis AN, Jones PJH \& Kubow, S. Lack of effect of Cuban sugar cane policosanols on LDL oxidation in hypercholesterolemic individuals. To be submitted.

6- Jones PJH, Kassis AN \& Marinangeli PF. Policosanol loses its luster as a cholesterol lowering agent.

Manuscript 1: the candidate's role in this study was sharing responsibilities in running the animal trial collecting and analyzing data. The candidate wrote the manuscript with the guidance and feedback from co-authors.

Manuscript 2: the candidate was involved in running the animal trial, collecting data as well as reviewing and providing feedback for the manuscript. 
Manuscript 3,4 and 5: the candidate ran the clinical trial, collected and analyzed data. The manuscripts were written by the candidate with guidance and feedback from co-authors. Manuscript 6: the candidate was involved in writing the manuscript.

I, Peter Jones, co-author of the fore-mentioned manuscripts, hereby agree to their inclusion in the $\mathrm{PhD}$ thesis of the candidate, Amira Kassis..

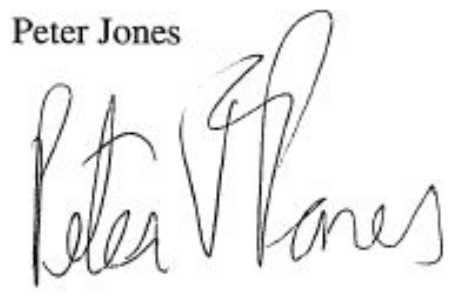




\section{Lack of cholesterol-lowering efficacy of Cuban sugar cane policosanols in hypercholesterolemic persons ${ }^{1-3}$}

Amira N Kassis and Peter JH Jones

ABSTRACT

Background: More than 50 studies have reported substantial reductions in plasma lipid concentrations in response to $2-40 \mathrm{mg}$ Cuban sugar cane policosanol (SCP) mixtures/d. However, several animal and human trials conducted outside of Cuba that used nonCuban mixtures have failed to reproduce the efficacy of policosanols observed in earlier studies.

Objective: The objective was to evaluate lipid-modulating actions of the authentic Cuban SCPs on plasma lipids in healthy hypercholesterolemic volunteers.

Design: Twenty-one volunteers consumed, under supervision, $10 \mathrm{mg} \mathrm{SCPs/d} \mathrm{or} \mathrm{a} \mathrm{placebo} \mathrm{incorporated} \mathrm{in} \mathrm{margarine} \mathrm{as} \mathrm{an} \mathrm{afternoon}$ snack, for a period of $28 \mathrm{~d}$ with the use of a randomized, double-blind crossover study design. Subjects maintained their habitual diet and physical activity and were weighed daily throughout the study period. Blood was collected at days 1,2,28, and 29 of the feeding trial, and lipid concentrations were measured.

Results: Body weights did not vary significantly throughout the trial and did not affect plasma lipid values. No significant difference was observed between treatment and control groups in plasma total, LDL-, HDL-cholesterol, and triacylglycerol concentrations. Conclusion: Present results show no beneficial effects of Cuban SCPs on lipid indicators in hypercholesterolemic persons and question the clinical usefulness of policosanol mixtures as cholesterollowering neutraceutical agents. Am J Clin Nutr 2006;84: 1003-8.

KEY WORDS Double-blind crossover study design, sugar cane policosanols, cholesterol-lowering effect, LDL cholesterol, hypercholesterolemic persons

\section{INTRODUCTION}

Sugar cane policosanols (SCPs) have repeatedly been shown to reduce plasma LDL cholesterol, decreasing the risk of cardiovascular incidents $(1-28)$. Clinical trials of varying durations tested the efficacy of SCP doses, ranging from 2 to $40 \mathrm{mg} / \mathrm{d}$ and involving subjects from various population groups $(14,18,21$, 24,25 ). Results show a reduction in total and LDL cholesterol of $13-23 \%$ and $19-31 \%$, respectively, as reviewed by Varady et al (29) in 2003. The lipid-lowering action of policosanols was compared with that of statins in a series of studies, showing similar efficacy for the 2 substances in terms of lowering total and LDL cholesterol $(9,15,17,19,25)$. In a recent meta-analysis that compared lipid-lowering activities of policosanols and plant sterols, a net reduction in LDL cholesterol of $24 \%$ was observed in the policosanol treatment groups compared with $10 \%$ in the plant sterol groups, compared with placebo (30).

All clinical trials that studied the lipid-lowering effect of policosanols were almost without exception conducted by one group of researchers based in Cuba. However, recently other investigators have contradicted the positive outcomes of the original research laboratory (31-34). Although similar to the authentic Cuban product, the policosanol mixtures used by those trials differed in source or composition of minor alcohols, possibly explaining the discrepancies between findings. Indeed, researchers initially providing data for the remarkable efficacy of SCPs suggested that failure by other groups to observe cholesterollowering effects could be explained by differences in the policosanol ratios in the mixtures tested (8). The purpose of the present study was to evaluate the authentic Cuban mixture of policosanols, as used in previous efficacy studies, as an ingredient for cholesterol lowering in moderately hypercholesterolemic persons.

\section{SUBJECTS AND METHODS}

\section{Subjects and treatments}

Otherwise healthy hypercholesterolemic men and postmenopausal women $(n=21)$, aged $40-80 \mathrm{y}$ and with body mass index (in $\mathrm{kg} / \mathrm{m}^{2}$ ) of $23-30$, were recruited for the clinical trial. The volunteers were asked to visit the research unit for 2 screenings, including a blood draw to measure their lipid profile and other health indicators and a medical examination. The subjects accepted in the study had plasma LDL-cholesterol concentrations that ranged from 3.0 to $5.0 \mathrm{mmol} / \mathrm{L}$ and triacylglycerol concentrations $<4.0 \mathrm{mmol} / \mathrm{L}$ at screening. Exclusion criteria included a history of recent or chronic use of oral hypolipidemic therapy or chronic use of insulin, systemic antibodies, corticosteroids, androgens, or phenytoin. Subjects were also excluded if they had experienced a myocardial infarction, coronary artery bypass, or other major surgical procedures within the past 6 mo or reported

\footnotetext{
${ }^{1}$ From the School of Dietetics and Human Nutrition, McGill University. Ste-Anne-de-Bellevue, Montréal, Quebec, Canada.

${ }^{2}$ Supported by a research grant from the Advanced Foods and Materials Network, Networks of Centres of Excellence.

${ }^{3}$ Address reprint requests to PJH Jones. School of Dietetics and Human Nutrition, McGill University, 21111 Lakeshore Rond. Ste-Anne-deBellevue, Quebec, Canada, H9X 3V9. E-mail: peter.jones @mcgill.ca. Received March 24, 2006 Accepted for publication July 5, 2006
} 
recent onset of angina, congestive heart failure, inflammatory bowel disease, pancreatitis, or hypothyroidism. Significant preexisting diseases, including cancer, chronic use of laxatives, smoking, or consumption of $>2$ drinks per day were also considered to be part of the exclusion criteria. Before they were enrolled in the study, the subjects were asked to sign a consent form that outlined the details of the trial. Policosanols derived from sugar cane wax (Lipex; Dalmer Laboratories, La Havana, Cuba) were purchased in the form of 5-mg pills which were crushed and incorporated into margarine. The composition of the SCP treatment was $65.6 \%$ octacosanol, $13.4 \%$ triacontanol, and $4.5 \%$ hexacosanol (unpublished data, 2006). The daily dose of treatment provided $10 \mathrm{mg}$ policosanols mixed with $10 \mathrm{~g}$ margarine and was served on a slice of bread.

\section{Protocol}

The feeding trial was designed as a double-blind crossover study, whereby subjects were randomly assigned to either policosanol or control margarines during 2 phases of $28 \mathrm{~d}$ each, separated by a washout phase of the same duration. The subjects were asked to maintain their habitual diet and physical activity patterns throughout the study. They were required to visit the clinic daily to consume the treatment as a late afternoon snack under staff supervision at the Mary Emily Clinical Research Centre, to ensure absolute compliance. The subjects were asked to abstain from alcohol throughout the 2 study phases, and caffeinated drinks were restricted to 1 cup/d. Body weights were recorded daily to monitor weight fluctuations throughout the study period. At the beginning and end of each feeding phase, blood draws were scheduled to check for health abnormalities at the onset of the trial and as a result of treatment. All procedures included in the protocol were approved by the ethics committee of the medical faculty of McGill University.

\section{Plasma lipid analyses}

Fasting blood samples were drawn from the subjects on days $1,2,28$, and 29 into EDTA-coated tubes and centrifuged at $805 \times$ $g$ for $20 \mathrm{~min}$ at $4{ }^{\circ} \mathrm{C}$. Then, plasma was separated from red blood cells and stored at $-80^{\circ} \mathrm{C}$ for further analysis. Initial lipid values were measured from blood samples taken on days 1 and 2 , whereas endpoint values were taken from days 28 and 29 . Plasma total, HDL-cholesterol, and triacylglycerol concentrations were measured with enzymatic kits, standardized reagents, and standards with the use of a VP Autoanalyzer (Abbott Laboratories, North Chicago, IL). The Friedewald equation was used to calculate LDL-cholesterol concentrations (35).

\section{Statistical analyses}

The sample size $(n=21)$ for the clinical trial was calculated to provide an $80 \%$ probability of detecting an anticipated difference in indicators tested of $20 \%$, by using a CV of $15-20 \%$. Effects of SCP treatment on indicators of interest were compared with control with the use of a one-factor analysis of covariance for crossover models and subsequent Scheffe's post hoc test. The effect of sequence, treatment period, and weight change was included in the model as covariates to test for potential confounders. Statistical significance was set at a level of $P<0.05$. All data was analyzed by using SAS statistical software for WINDOWS version 8.02 (SAS Institute Inc, Cary, NC).
TABLE 1

Baseline characteristics of the subjects ${ }^{7}$

\begin{tabular}{lccc}
\hline \hline Variable & $\begin{array}{c}\text { Men } \\
(n=12)\end{array}$ & $\begin{array}{c}\text { Women } \\
(n=9)\end{array}$ & $\begin{array}{c}\text { All } \\
(n=21)\end{array}$ \\
\hline Age $(\mathrm{y})$ & $54.0 \pm 2.9^{\mathrm{a}}$ & $60.1 \pm 2.6^{\mathrm{a}}$ & $57.8 \pm 2.1$ \\
Weight $(\mathrm{kg})$ & $84.8 \pm 4.9^{\mathrm{a}}$ & $71.1 \pm 3.8^{\mathrm{b}}$ & $76.3 \pm 3.2$ \\
BMI $\left(\mathrm{kg} / \mathrm{m}^{2}\right)$ & $26.9 \pm 1.0^{\mathrm{a}}$ & $26.3 \pm 0.8^{\mathrm{a}}$ & $26.5 \pm 0.6$ \\
Tocal cholesterol $(\mathrm{mmol} / \mathrm{L})$ & $5.4 \pm 0.4^{\mathrm{a}}$ & $6.09 \pm 0.3^{\mathrm{a}}$ & $5.8 \pm 0.2$ \\
LDL cholesterol $(\mathrm{mmol} / \mathrm{L})$ & $3.5 \pm 0.3^{\mathrm{a}}$ & $3.8 \pm 0.3^{\mathrm{a}}$ & $3.7 \pm 0.2$ \\
HDL cholesterol $(\mathrm{mmo} / \mathrm{L})$ & $1.2 \pm 0.1^{\mathrm{a}}$ & $1.6 \pm 0.1^{\mathrm{b}}$ & $1.4 \pm 0.09$ \\
Triacylglycerols $(\mathrm{mmol} / \mathrm{L})$ & $1.5 \pm 0.4^{\mathrm{a}}$ & $1.7 \pm 0.3^{\mathrm{a}}$ & $1.6 \pm 0.2$ \\
\hline
\end{tabular}

${ }^{7}$ All values are $\bar{x} \pm$ SEM. The men-to-women ratio was 1.3 . Values in a row with different superscript letters are significantly different, $P<0.05$.

\section{RESULTS}

\section{Subjects and dropout rate}

Twenty-two subjects were enrolled in the clinical trial. Baseline characteristics, recorded the first $2 \mathrm{~d}$ of the trial, are shown in Table 1. One subject dropped out because of conflicting schedules. Therefore, 21 subjects successfully completed the feeding trial. Most subjects tolerated the afternoon snack well. However, 4 subjects reported abdominal pain, soft stools, and increased frequency of bowel movements. Two of these subjects were consuming the treatment and the other 2 were receiving the placebo.

Blood biochemistry and weight changes across the study period

Blood biochemistry and hematology values remained within normal ranges throughout the study period. Initial and final body weights were calculated as average weights of the first and last $5 \mathrm{~d}$ of each phase, respectively. These averages are presented in Figure 1 and show that subjects maintained a stable weight throughout the study phases. In fact, average final body weights varied by $<2 \mathrm{~kg}$ from the initial weight during the 2 treatment periods. Only one participant, subject 4 , lost $3 \mathrm{~kg}$ during the control phase.

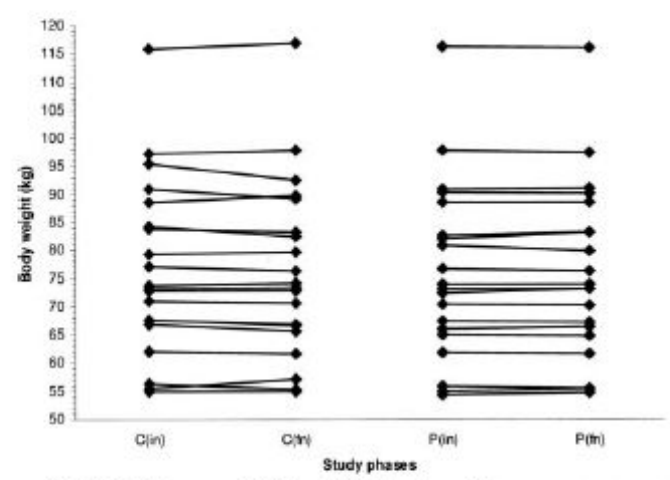

FIGURE 1. Average initial and final body weights across treatment phases. $\mathrm{C}(\mathrm{in})$, control phase initial value; $\mathrm{C}(\mathrm{fn})$, control phase final value: $\mathrm{P}(\mathrm{in})$, policosanol phase initial value; $\mathrm{P}(\mathrm{fn})$, policosanol phase final value. 

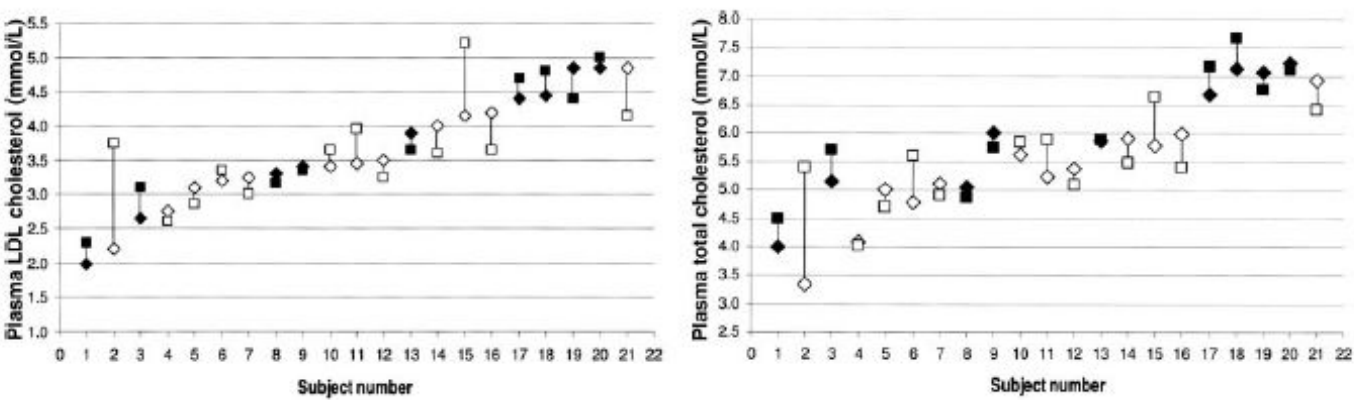

Subject number
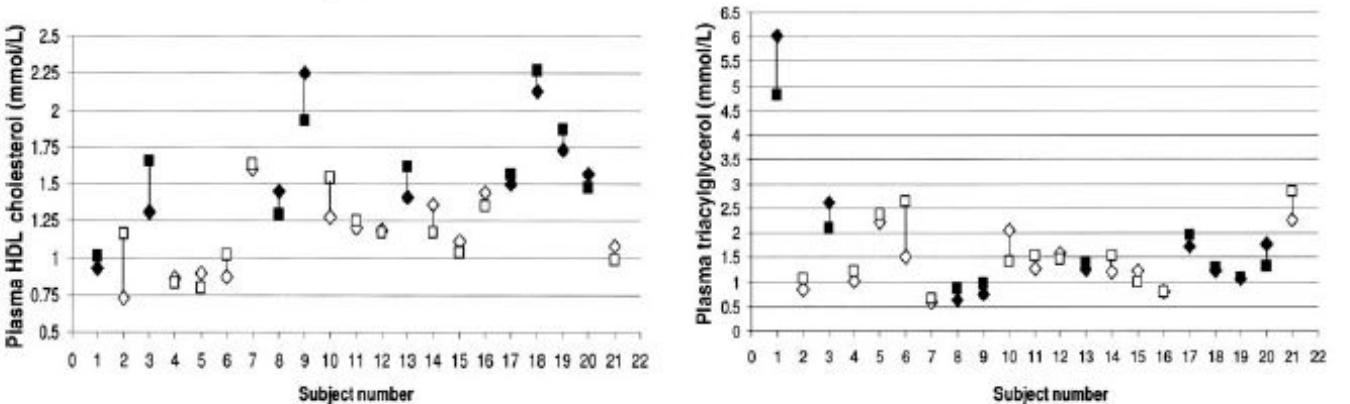

FIGURE 2. Subjects' plasma lipid endpoints in response to sugar cane policosanol (SCP) treatment and control. Individual plasma total cholesterol, LDL-cholesterol, HDL-cholesterol, and triacylglyoerol values in response to placebo in men ( $\diamond)$ and women ( $\bullet$ ) and in response to SCP treatment in men ( $\square$ ) and women $(\mathbf{W})(n=21)$

Plasma lipid concentrations across the study period

\section{Total cholesterol responses to treatment}

Individual total cholesterol values across treatment phases are shown in Figure 2. Authentic SCPs did not significantly affect plasma total cholesterol concentration endpoints or percent changes across each phase compared with control. Mean ( \pm SEM) plasma total cholesterol was $5.77 \pm 0.21 \mathrm{mmol} / \mathrm{L}$ at the end of the SCP treatment phase compared with $5.60 \pm$ $0.21 \mathrm{mmol} / \mathrm{L}$ in response to placebo $(P=0.25)$. Percent changes from baseline were $(P=0.18) 1.8 \pm 3.0 \%$ and $-4.0 \pm 3.0 \%$ in the SCP and control groups, respectively.

\section{$L D L$-cholesterol responses to treatment}

Calculated LDL-cholesterol endpoints and percent changes were not significantly affected by SCP supplementation. Individual responses to treatment and control are presented in Figure 2. Mean ( \pm SEM) LDL-cholesterol values for SCP treatment and control groups were $(P=0.52) 3.69 \pm 0.18 \mathrm{mmol} / \mathrm{L}$ and $3.61 \pm$ $0.18 \mathrm{mmol} / \mathrm{L}$, respectively. Average LDL-cholesterol values were higher than baseline by $4.5 \pm 4.0 \%$ in response to the SCP treatment and $1.6 \pm 4.0 \%$ lower than baseline in response to control. However, differences between the groups did not reach significance $(P=0.28)$

\section{$H D L$-cholesterol responses to treatment}

SCP treatment did not show any significant effect on HDLcholesterol endpoints or percent changes compared with control. Each subject's HDL-cholesterol responses to treatments are shown in Figure 2. Mean ( \pm SEM) endpoint values for SCP and control groups were $(P=0.36) 1.37 \pm 0.81 \mathrm{mmol} / \mathrm{L}$ and $1.33 \pm$ $0.81 \mathrm{mmol} / \mathrm{L}$, respectively. Percent changes in HDL cholesterol were $(P=0.19)-2.7 \pm 3.0 \%$ in the SCP group compared with $-7.4 \pm 3.0 \%$ in the control group.

\section{Triacylglycerol responses to treatment}

As for the other lipid indicators, no significant effect of SCPs was observed on plasma triacylglycerol endpoints $(P=0.72)$ and percent changes $(P=0.36)$. Individual triacylglycerol responses to treatment and control are shown in Figure 2. Mean ( \pm SEM) endpoint values for treatment and control groups were $1.63 \pm$ $0.24 \mathrm{mmol} / \mathrm{L}$ and $1.59 \pm 0.24 \mathrm{mmol} / \mathrm{L}$, respectively; percent change in the SCP group was $-3.2 \pm 6.0 \%$ from baseline compared with $-4.3 \pm 6.0 \%$ for the control group.

Effect of sequence, period, and body weight change on lipid indicators

When study sequence, treatment period, and weight changes across periods were included in the statistical model as covariates, no significance was recorded $(P>0.05)$ in their effect on plasma mean endpoint values and percent changes of total, LDL-cholesterol, HDL-cholesterol, and triacylglycerol concentrations.

\section{DISCUSSION}

Contradicting the previous literature on Cuban SCPs, the results of the present study do not show that authentic SCPs alter 
total cholesterol, LDL-cholesterol, HDL-cholesterol, or triacylglycerol concentrations in healthy, hypercholesterolemic persons. Original research on the effect of SCPs on plasma lipids in humans showed highly promising results in terms of risk reduction for coronary heart disease (1-28). Tested on numerous study populations and for various durations, $\mathrm{SCP}$ treatments proved to be effective regardless of diet control and compliance monitoring. In fact, SCPs were often tested in a free-living context in which subjects were instructed to follow a National Cholesterol Education Program Step 1 diet and were given the treatment for consumption under no supervision $(9,16,17,24)$. In addition, follow-up visits, including tablet counting, were scheduled once a month $(2,9,10,15,24)$, in some cases once every 3 mo $(20)$. Still, doses of SCPs as low as $2 \mathrm{mg} / \mathrm{d}$ were seen to reduce total and LDL cholesterol by $14.8 \%$ and $15.6 \%$, respectively (2). The design of the present study was such that $10 \mathrm{mg} \mathrm{SCPs} / \mathrm{d}$ were administered under staff supervision, toensure that subjects were following the study protocol in terms of treatment dose and time of consumption. On the one hand, we were not able to replicate the results obtained by previous research on authentic Cuban SCPs. On the other hand, the lack of efficacy seen in this trial agrees with the datareported by the few human studies conducted outside the original research laboratory. Lin et al (33) used $20 \mathrm{mg}$ wheat-germ policosanols/d with similar octacosanol content to the authentic mixture for a period of $4 \mathrm{wk}$. No benefit was attributed to the effect of policosanols on blood lipids. In 2 recent randomized double-blind placebo-controlled crossover trials $(31,32), 10 \mathrm{mg}$ rice policosanols/d was administered to hypercholesterolemic subjects for a period of $8 \mathrm{wk}$. Although the treatment significantly reduced total cholesterol, it did not affect LDL cholesterol, HDL cholesterol, or triacylglycerols. Because the cholesterol-lowering effect of SCPs is mainly mediated by a decrease in LDL cholesterol (36), significant reductions would be expected as can be observed in original policosanol studies. A longer duration of treatment was used in a recent crossover stud by Greyling et al (34), in which participants received $20 \mathrm{mg}$ Lesstanol Octa-60 policosanols/d for a period of $12 \mathrm{wk}$. Agreeing with results of a previous animal trial on the same product (37), the investigators reported no significant effect on total and LDL cholesterol in hypercholesterolemic humans.

One of the main points of controversy in policosanol research is the appropriateness of the product used in studies yielding negative results. Octacosanol is the main alcohol in policosanol mixtures and was suggested to be the main active component of the treatments (38). The octacosanol content of wheat germ, rice, and Lesstanol Octa-60 policosanols is similar (33) or close (31, 34) to the authentic product. However, mixtures with diverging minor alcohol compositions appear to have different lipidlowering activities despite their similar octacosanol contents (8). To date, the original policosanol mixture was used in only one clinical trial (39), in which the raw material was provided by Dalmer Laboratories and the tablets were manufactured in Germany. Results from that recent trial agree with conclusions of external studies that found no effect of policosanols on the lipid profile in hypercholesterolemic patients. Consequently, in the present study, the use of authentic SCP tablets manufactured in Cuba is of great importance to compare our results with those of the original research.

Dissimilarities between original and external research on policosanol include the length of the study period. Unlike external clinical trials with durations ranging from 4 to $12 \mathrm{wk}(31-34,39)$, original researchers tested SCPs in the context of clinical trials covering 6 wk to 3 y $(1-5,9,10,12,15,17,19,23)$. However. note that, when interim lipid measurements were performed, SCP efficacy was already manifested by the fourth week of the trial $(15,19)$. The present study did not show any changes in plasma lipids at the end of the 4-wk trial, therefore agreeing with external researchers on the inefficacy of policosanols

The form of administration of a treatment often plays a role in determining its efficacy. Most studies conducted in Cuba used policosanol treatments in the form of a pill ingested with a meal. The objective of this study was to evaluate SCPs incorporated in a food; therefore, policosanols were mixed in $10 \mathrm{~g}$ margarine. Fatty matrices, especially margarine, have been used as a vehicle for fat-soluble substances such as plant sterols $(40,41)$. Previous literature shows that these matrices resulted in better cholesterol lowering than did others, such as low-fat beverages and capsules $(42,43)$. Knowing that policosanols are not soluble in water (44), the fatty matrix used was considered appropriate for better mixing and distribution of SCPs. Therefore, the lack of efficacy seen in the present study is not likely to be related to the form of administration of policosanols.

Very-long-chain fatty alcohols are rather abundant in nature, more precisely in plants and oils that constitute a significant part of the human diet (45). Specific human intakes of very-longchain fatty alcohols are yet to be determined; however, estimates of the typical US intake of the wax ester hexacosanoate are reported to be $12-40 \mathrm{mg} / \mathrm{d}(45)$. Octacosanol is found in many common market foods, such as rice, wheat, apples, plums, spinach, and various nuts and seeds (45); therefore, a diet already providing a high concentration of octacosanol could potentially mask the effect of a supplementation of this alcohol. Investigating variations in dietary intakes of policosanols in general and octacosanol in particular across populations may determine the involvement of an important factor (ie, the typical octacasanol content of traditional foods consumed). Furthermore, the use of Cuban subjects in original positive research compared with most European or North American participants in external negative research draws the attention to the possible effect of genetics or dietary habits on policosanol action, potentially making this treatment specific to certain populations.

Despite the extensive research published on the efficacy of SCPs, their mechanism of action is still unclear. SCPs are believed to be absorbed by the gut (46) and transported to the hepatocytes where they reduce cholesterol biosynthesis by suppressing 3-hydroxy-3-methylglutaryl coenzyme A reductase $(47,48)$ and increasing LDL uptake by the cells $(49)$. Radioisotope labeling was used in most studies that looked at policosanol absorption and their effect on cholesterol synthesis. In both cases, labeling policosanols could lead to erroneous results because of their degradation in the body into their metabolites (50). This would suggest that this detection method is nonspecific. Also, research has reported poor absorption rates for policosanols in the gut, possibly because of their extreme hydrophobicity ( 45 . 46,51 ). To understand the mechanism by which a significant amount of SCPs can reach the hepatocytes and directly or indirectly induce changes in cholesterol synthesis, more research on the alcohols' bioavailability and their effect on cholesterol kinetics is necessary.

In conclusion, the present study did not find SCPs to be efficacious for the reduction of plasma cholesterol in hypercholesterolemic persons. Additional studies are needed to fully understand 
their mechanism of action, their metabolism in the body, and the genetic and dietary factors affecting their action to determine the reasons for controversy in policosanol research.

We thank the study participants for their good will and compliance throughout the trial period.

ANK designed the study, collected and analyzed the data, and wrote the manuscript. PJHJ designed the study, supervised and provided significant advice and consultation, and wrote the manuscript. None of the authors had any conflicts of interest.

\section{REFERENCES}

1. Aneiros E, Mas R, Calderon B, et al. Effect of policosanol in lowering cholesterol levels in patients with type II hypercholesterolemia. Cun Ther Res Exp 1995:56:176-82.

2. Batista J, Stusser R, Saez F, Perez B. Effect of policossnol on hyperlipidemia and coronary heart disease in middle-aged patients. A 14 month pilot study. Int J Clin Pharmacol Ther 1996:34:134-7.

3. Canetti M, Moreira M, Mas R, Illnait J, Femandez L, Fernandez JC. Effects of policosanol on primary hypercholesterolemia: a 3-year openextension follow-up. Curr Ther Res Clin Exp 1997;58:868-75.

4. Canetti M. Moreira M. Illnait J, et al. One-year study of the effect of policosanol on lipid profile in patients with type II hypercholesterolemia. Adv Ther 1995; 12:245-54.

5. Canetti M, Moreira M, Mas R, etal. A two-year study on the efficacy and tolerability of policosanol in patients with type II hyperlipoproteinaemia. Int J Clin Pharmacol Res 1995; 15:159-65.

6. Castano G, Canetti M, Moreira M. Efficacy and tolerability of policosanol in elderly patients with type II hypercholesterolemia: a 12-month study. Curr Ther Res Clin Exp 1995:56:819-28.

7. Castano G, Mas R, Nodarse M. Illnait J, Fernandez JC. One-year of the efficacy and safety of policosanol ( $5 \mathrm{mg}$ twice daily) in the treatment of type II hypercholesterolemia. Curr Ther Res Clin Exp 1995:56:296303.

8. CastanoG, Fernandez L, Mas R, et al.Comparison of the efficacy, safety and tolerability of original policcsanol versus other mixtures of higher aliphatic primary al cohols in patients with type II hypercholesterol emia. Int J Clin Pharmacol Res 2002:22:55-66.

9. Castano G, Fernandez L, Mas R, Illnait J, Mesa M, Femandez J. Compurison of the effects of policosanol and atorvastatin on lipid profile and platelet aggregation in patients with dyslipidemia and type 2 diabetes platelet aggregation in patients with dyslipi
mellitus. Clin Drug Invest 2003:23:639-50.

10. Castano G, Mas Ferreiro R, Fernandez L, Gamez R, Illnait J, Fernandez C. A long-term study of policosanol in the treatment of intermittent claudication. Angiology 2001:52:115-25.

11. Castano G, Mas R. Arruzazabala ML, et al. Effects of policosanol and pravastatin on lipid profile, platelet aggregation and endothelemia in older hypercholesterolemic patients. Int J Clin Pharmacol Res 1999:19. $105-16$.

12. Castano G. Mas R, Fernandez JC, Illnait J, Femandez L. Alvarez E. Effects of policosanol in older patients with type II hypercholesterolemia and high coronary risk. J Gerontol A Biol Sci Mod Sci 2001:56:M18692.

13. Castano G, Mas R, Fernandez L. et al. Effects of policosanol on postmenopausal women with type II hypercholesterolemia. Gynecol Endocrinol 2000; 14:187-95.

14. Castano G, Mas R, Femandez L. Illnait J, Gamez R, Alvarez E. Effects of policosanol 20 versus $40 \mathrm{mg} /$ day in the treatment of patients with type II hypercholesterolemia: a 6-month double-blind study. Int J Clin Pharmacol Res 2001:21:43-57

15. Castano G, Mas R, Femandez L, et al. Comparison of the efficacy and tolerability of policosanol with atorvastatin in elderly patients with type II hypercholesterolaemia. Drugs Aging 2003:20:153-63.

16. Castano G, Mas R, Gamez R, et al. Concomitant use of policosanol and beta-blockers in older patients. Int J Clin Phamacol Res 2004:24:65-77.

17. Castano G, Menendez R, Mas R, et al. Effects of policosanol and lovastatin on lipid profile and lipid peroxidation in patients withdys lipidemia associated with type 2 diabetes mellitus. Int J Clin Phamacol Res 2002 22:89-99

18. CrespoN, Alvarez R, Mas R, Illnait J, Fernandez L, Fernandez JC. Effect of policosanol on patients with non-insulin-dependent diabetes mellitus and hypercholesterolemia: a pilot study. Curr Ther Res 1997;58:44-51.
19. Fernandez J, Mas R, Castano G, et al. Comperison of the efficacy, safety and tolerability of policosanol versus Fluvastatin in eldenly hypercholesterolemic women. Clin Drug Invest 2001:21:103-13.

20. Mas R. Castano G. Femandez L. Illnait J. Fernandez J. Alvarez E Clinical use: effects of policosanol on lipid profile and cardiac events in older hypencholesterolaemic patients with coronary disease. Clin Drug Invest 2001;21:485-99.

21. Castano G, Menendez R, Mas R, et al. Effects of policosanol and lovastatin on lipid profile and lipid peroxidation in patients withdyslipidemi associated with type 2 diabetes mellitus. Int J Clin Phamacol Res 2002: associated $22: 89-99$.

22. CrespoN Alvarez R, Mas R, Illnait J, Fernandez L, Femandez JC. Effoct of policosanol on patients with non-insulin-dependent diabetes mellitus and hypercholesterolemia: a pilot study. Curr Ther Res. 1997;58:44-51

23. Mas R, Castano G, Illnait J, et al. Effects of policosanol in patients with type II hypercholesterolemia and additional coronary risk factors. Clin Pharmacol Ther 1999:65:439-47.

24. Mirkin A, Mas R, Martinto M, et al. Efficacy and tolerability of policosanol in hypercholesterolemic postmenopausal women. Int J Clin Pharmacol Res 2001:21:31-41.

25. Ortensi G, Gladstein J, Valli H, Testone P. A comparative study of policosanol versus simvastatin in elderly patients with hypercholesterolemia. Curr Ther Res Clin Exp 1997;58:390-401.

26. Pons P, Mas R, Illnait J, et al. Efficacy and safety of policosanol in patients with primary hypercholesterolemia. Curr Ther Res Clin Exp 1992:52:507-13.

27. Pons P, Rodriguez M, Mas R, et al. One-year efficacy and safety of policosanol in patients with type II hypercholesterolemia. Curr Ther Res Clin Exp 1994:55: 1084-92.

28. Pons P, Rodriguez M, Robaina C. Effects of successive dose increases of policosanol on the lipid profile of patients with type II hypercholesterdaemia and tolerability to treatment. Int JClin Pharmacol Res 1994:14 $27-33$

29. Varady KA, Wang Y, Jones PJ. Role of policosanols in the prevention and treatment of cardiovascular disease. Nutr Rev 2003;61:376-83.

30. Chen JT, Wesley R. Shamburek RD, Pucino F, Csako G. Meta-analysis of natural therapies for hypertipidemia: plant sterols and stanols versus policosanol. Pharmacoherapy 2005;25:171-83.

31. Reiner Z, Tedeschi-Reiner E, Romic Z. Effects of rice policosanol on serum lipoproteins, homocysteine, fibrinogen and C-reactive protein in hypercholesterolaemic patients. Clin Drug Invest 2005;5:701-7.

32. Tedeschi-Reiner E, Reiner Z, Romic Z, Ivankovic D. A randomized, double-blind, placebo-controlled study of the antilipemic efficacy and tolerability of food supplement policosanol in patients with moderate hypercholesterolemia. Lijec Vjesn 2005:127:273-9 (in Croatian).

33. Lin Y, Rudrum M, van der Wielen RP, et al. Wheat germ policosanol failed to lower plasma cholesterol in subjects with normal to mildly elevated cholesterol concentrations. Metabolism 2004,53:1309-14.

34. Greyling A, De Witt C, Oosthuizen W, Jerling JC. Effects of a policosanol supplement on serum lipid concentrations in hypercholesterolaemic and heterozygousfamilial hypercholesterolaemic subjects. BrJNutr 2006:95:968-75.

35. Friedewald WT Levy RI. Friedrickson DS. Estimation of the concentration of low-density lipoprotein cholesterol in plasma without use of preparative ultracentrifuge. Clin Chem 1972;18:499-502.

36. Menendez R, Amor AM, Gonzalez RM, Fraga V, Mas R. Effoct of policosanol on the hepatic cholesterol biosynthesis of normocholesterolemic rats. Biol Res 1996:29:253-7.

37. Murphy KJ, Saint DA. Howe PR. Lack of effect of sugar cane and sunflower seed policosanols on plasma cholesterol in rabbits. Asia Pac J Clin Nutr 2004:13(suppl):S69 (abstr).

38. Arruzazabala ML, Carbajal D, Mas R, Molina V, Castano G, Gamez R. Effects of D-003, a new compound purified from sugarcane wax, on platelet aggregation in healthy volunteers. Clin Drug Invest 2003;23. $107-18$.

39. Berthold HK, Unverdorben S, Degenhardt R, Bulitta M, Gouni-Berthold I. Effect of policosanol on lipid levels among patients with hypercholesterolemia or combined hyperlipidemia: a randomized controlled trial. JAMA 2006:295:2263-9.

40. Jones PJ, Ntanios FY, Raeini-Sarjaz M, Vanstone CA. Cholesterollowering efficacy of a sitostanol-containing phytosterol mixture with a prudent diet in hyperlipidemic men. Am J Clin Nutr 1999;69:403-10.

41. Vanstone CA, Raeini-Sarjaz M. Parsons WE, Jones PJ. Unesterified 
plant sterols and stanols lower LDL-cholesterol concentrations equivalently in hypercholesterolemic persons. Am J Clin Nutr 2002:76. 1272-8.

42. Denke MA. Lack of efficacy of low-dose sitostanol therapy as an adjunct to a cholesterol-lowering diet in men with moderate hypercholesterolemia. Am J Clin Nutr 1995;61:392-6.

43. Jones PJ, Vanstone CA. Raeini-Sarjaz M. St-Onge MP. Phytosterols in low- and nonfatbeverages as part of a controlleddiet fail to lower plasma lipid levels. Am J Clin Nutr 2003;69:1144-50.

44. Uribarri E, Laguna A, Sierra R, Ricardo Y. Physico-mechanical characterization of policosanol, a novel hypocholesterolemic drug. Drug Dev Ind Pharm 2002:28:89-93.

45. Hargrove JL, Greenspan P, Hartle DK. Nutritional significance and metabolism of very long chain fatty alcohols and acids from dietary waxes. Exp Biol Med (Maywood) 2004:229:215-26.

46. Kabir Y. Kimura S. Tissue distribution of (8-14C)-octacosanol in liver and muscle of rats after serial administration. Ann Nutr Metab 1995:39: $279-84$.

47. Menendez R, Amor AM. Rodeiro I, et al. Policosanol modulates HMG CoA reductase activity in culturod fibroblasts. Arch Med Res 2001;32 $8-12$.

48. Menendez R, Arruzazabala L, Mas R, et al. Cholesterol-lowering effect of policosanol on rabbits with hypercholesterolaemia induced by a wheat starch-casein diet. Br J Nutr 1997:77:923-32

49. Menendez R, Arruzazabala L, Mas R, et al. Cholesterol-lowering effect of policosanol on rabbits with hypercholesterolaemia inducod by a wheat starch-casein diet. Br J Nutr 1997:77:923-32.

50. Mannaerts GP, Van Veldhoven PP, Casteels M. Peroxisomal lipid degradation via beta- and alpha-oxidation in mammals. Cell Biochem Biophys 2000;32:73-87.

51. Menendez R, Marrero D, Mas R, Femandez I, Gonzalez L, Gonzalez RM. In vitro and in vivo study of octacosanol metabolism. Arch Med Res 2005:36:113-9. 


\section{Manuscript 4-Published}

Changes in cholesterol synthesis and absorption following sugar cane policosanol supplementation in hypercholesterolemic individuals

Amira N. Kassis and Peter J.H. Jones

Published in Lipids in Health and Disease 
September 17, 2007

To Dr. Peter Jones

The purpose of this letter is to confirm that Dr. Peter Jones, co-author of the below mentioned manuscripts, agrees to their inclusion in the thesis of Amira Kassis, $\mathrm{PhD}$ candidate.

\section{Manuscripts to be included}

1- Kassis AN, Marinangeli CP, Jain D, Ebine N \& Jones PJ (2006) Lack of effect of sugar cane policosanol on plasma cholesterol in golden Syrian hamsters.

Atherosclerosis. Epub ahead of print

2- Marinangeli CP, Kassis AN, Jain D, Ebine N, Cunnane SC \& Jones PJ (2007)

Comparison of composition and absorption of sugarcane policosanols. Br J Nutr. Feb;97(2):381-8

3- Kassis AN \& Jones PJ (2006) Lack of cholesterol-lowering efficacy of Cuban sugar cane policosanols in hypercholesterolemic persons. Am J Clin Nutr. 2006 Nov;84(5):10

4- Kassis AN \& Jones PJH. Changes in cholesterol metabolism in response to sugar cane policosanol in hypercholesterolemic individuals. To be submitted.

5- Kassis AN, Jones PJH \& Kubow, S. Lack of effect of Cuban sugar cane policosanols on LDL oxidation in hypercholesterolemic individuals. To be submitted.

6- Jones PJH, Kassis AN \& Marinangeli PF. Policosanol loses its luster as a cholesterol lowering agent.

Manuscript 1: the candidate's role in this study was sharing responsibilities in running the animal trial collecting and analyzing data. The candidate wrote the manuscript with the guidance and feedback from co-authors.

Manuscript 2: the candidate was involved in running the animal trial, collecting data as well as reviewing and providing feedback for the manuscript. 
Manuscript 3,4 and 5: the candidate ran the clinical trial, collected and analyzed data. The manuscripts were written by the candidate with guidance and feedback from co-authors. Manuscript 6: the candidate was involved in writing the manuscript.

I, Peter Jones, co-author of the fore-mentioned manuscripts, hereby agree to their inclusion in the $\mathrm{PhD}$ thesis of the candidate, Amira Kassis..

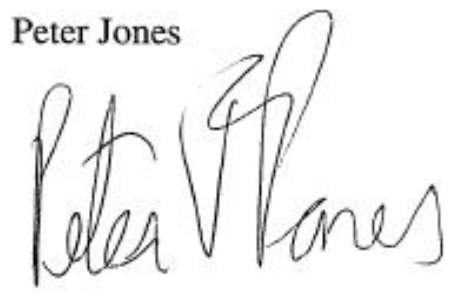




\section{Manuscript 5-To be submitted}

Lack of effect of sugar cane policosanols on LDL oxidation in hypercholesyerolemic individuals

Amira N. Kassis, Stan Kubow and Peter J.H.Jones 
September 17, 2007

To Dr. Peter Jones

The purpose of this letter is to confirm that Dr. Peter Jones, co-author of the below mentioned manuscripts, agrees to their inclusion in the thesis of Amira Kassis, $\mathrm{PhD}$ candidate.

\section{Manuscripts to be included}

1- Kassis AN, Marinangeli CP, Jain D, Ebine N \& Jones PJ (2006) Lack of effect of sugar cane policosanol on plasma cholesterol in golden Syrian hamsters.

Atherosclerosis. Epub ahead of print

2- Marinangeli CP, Kassis AN, Jain D, Ebine N, Cunnane SC \& Jones PJ (2007)

Comparison of composition and absorption of sugarcane policosanols. Br J Nutr. Feb;97(2):381-8

3- Kassis AN \& Jones PJ (2006) Lack of cholesterol-lowering efficacy of Cuban sugar cane policosanols in hypercholesterolemic persons. Am J Clin Nutr. 2006 Nov;84(5):10

4- Kassis AN \& Jones PJH. Changes in cholesterol metabolism in response to sugar cane policosanol in hypercholesterolemic individuals. To be submitted.

5- Kassis AN, Jones PJH \& Kubow, S. Lack of effect of Cuban sugar cane policosanols on LDL oxidation in hypercholesterolemic individuals. To be submitted.

6- Jones PJH, Kassis AN \& Marinangeli PF. Policosanol loses its luster as a cholesterol lowering agent.

Manuscript 1: the candidate's role in this study was sharing responsibilities in running the animal trial collecting and analyzing data. The candidate wrote the manuscript with the guidance and feedback from co-authors.

Manuscript 2: the candidate was involved in running the animal trial, collecting data as well as reviewing and providing feedback for the manuscript. 
Manuscript 3,4 and 5: the candidate ran the clinical trial, collected and analyzed data. The manuscripts were written by the candidate with guidance and feedback from co-authors. Manuscript 6: the candidate was involved in writing the manuscript.

I, Peter Jones, co-author of the fore-mentioned manuscripts, hereby agree to their inclusion in the $\mathrm{PhD}$ thesis of the candidate, Amira Kassis..

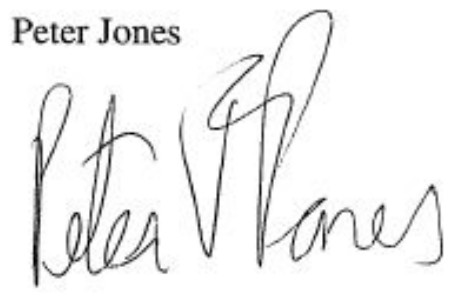




\section{To Dr. Stan Kubow}

The purpose of this letter is to confirm that Dr. Stan Kubow, co-author of the below mentioned manusiript, agrees to its inclusion in the thesis of Amira Kassis, $\mathrm{PhD}$ candidate.

\section{Manuscript to be included}

Kassis AN, Kubøw S \& Jones PJH. Lack of effect of Cuban sugar cane policosanol on LDL oxidation in hypercholesterolemic individuals. To be submitted.

The candidate's tole in this study was to run the clinical trial, collect and analyze data. The manuscript 'was written by the candidate with guidance and feedback from coauthors.

I, Stan Kubow, co-author of the aforementioned manuscript, hereby agree to its inclusion in the $\mathrm{PhD}$ thesis of the candidate, Amira Kassis.

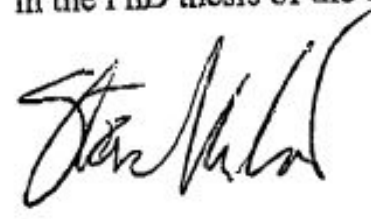

Stan Kubow 


\section{Editorial-To be submitted}

\section{Policosanols Lose their Lustre as Cholesterol Lowering Agents}

Peter J.H. Jones, Amira N. Kassis and Christopher P.F.Marinangeli 
September 17, 2007

To Dr. Peter Jones

The purpose of this letter is to confirm that Dr. Peter Jones, co-author of the below mentioned manuscripts, agrees to their inclusion in the thesis of Amira Kassis, $\mathrm{PhD}$ candidate.

\section{Manuscripts to be included}

1- Kassis AN, Marinangeli CP, Jain D, Ebine N \& Jones PJ (2006) Lack of effect of sugar cane policosanol on plasma cholesterol in golden Syrian hamsters.

Atherosclerosis. Epub ahead of print

2- Marinangeli CP, Kassis AN, Jain D, Ebine N, Cunnane SC \& Jones PJ (2007)

Comparison of composition and absorption of sugarcane policosanols. Br J Nutr. Feb;97(2):381-8

3- Kassis AN \& Jones PJ (2006) Lack of cholesterol-lowering efficacy of Cuban sugar cane policosanols in hypercholesterolemic persons. Am J Clin Nutr. 2006 Nov;84(5):10

4- Kassis AN \& Jones PJH. Changes in cholesterol metabolism in response to sugar cane policosanol in hypercholesterolemic individuals. To be submitted.

5- Kassis AN, Jones PJH \& Kubow, S. Lack of effect of Cuban sugar cane policosanols on LDL oxidation in hypercholesterolemic individuals. To be submitted.

6- Jones PJH, Kassis AN \& Marinangeli PF. Policosanol loses its luster as a cholesterol lowering agent.

Manuscript 1: the candidate's role in this study was sharing responsibilities in running the animal trial collecting and analyzing data. The candidate wrote the manuscript with the guidance and feedback from co-authors.

Manuscript 2: the candidate was involved in running the animal trial, collecting data as well as reviewing and providing feedback for the manuscript. 
Manuscript 3,4 and 5: the candidate ran the clinical trial, collected and analyzed data. The manuscripts were written by the candidate with guidance and feedback from co-authors. Manuscript 6: the candidate was involved in writing the manuscript.

I, Peter Jones, co-author of the fore-mentioned manuscripts, hereby agree to their inclusion in the $\mathrm{PhD}$ thesis of the candidate, Amira Kassis..

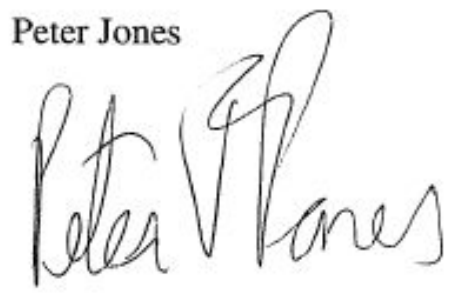


September 17, 2007

To Christopher Marinangeli,

The purpose of this letter is to confirm that Christopher Marinangeli, co-author of the below mentioned manuscripts, agrees to their inclusion in the thesis of Amira Kassis, $\mathrm{PhD}$ candidate.

\section{Manuscripts to be included}

1- Kassis AN, Marinangeli CP, Jain D, Ebine N \& Jones PJ (2006) Lack of effect of sugar cane policosanol on plasma cholesterol in golden Syrian hamsters.

Atherosclerosis. Epub ahead of print

2- Marinangeli CP, Kassis AN, Jain D, Ebine N, Cunnane SC \& Jones PJ (2007)

Comparison of composition and absorption of sugarcane policosanols. Br J Nutr. Feb;97(2):381-8

3- Jones PJH, Kassis AN, Marinangeli PF. Policosanol loses its luster as a cholesterol lowering agent. To be submitted.

Manuscript 1: the candidate's role in this study was sharing responsibilities in running the animal trial collecting and analyzing data. The candidate wrote the manuscript with the guidance and feedback from co-authors.

Manuscript 2: the candidate was involved in running the animal trial, collecting data as well as reviewing and providing feedback for the manuscript.

Manuscript 3: the candidate was involved in writing the manuscript.

I, Christopher Marinangeli, co-author of the aforementioned manuscripts, hereby agree to their inclusion in the $\mathrm{PhD}$ thesis of the candidate, Amira Kassis.

$$
\text { (P) Painangeh. }
$$

Christopher Marinangeli 